\title{
Updated checklist of Chinese Umbelliferae: nomenclature, synonymy, typification, distribution
}

\author{
M. G. Pimenov \\ Botanical Garden, Lomonosov Moscow State University, Vorobjevy Gory, Moscow, 117234, Russia. E-mail: mgpimenov@mail.ru
}

Key words: Apiaceae, carrot family, China, East Asia, systematics, taxonomy, phytogeography, endemic species, new combinations and synonyms, lectotypification.

Summary. The country with the highest taxonomic diversity in the Umbelliferae at the specific level, China possesses 657 species and 44 infraspecific taxa (in 110 genera) of the family, among all countries of the world. The number of endemic species is also considerable - 355. The main center of diversity of the Umbelliferae is SW part of the country, with 357 species, including 126 endemic ones. This center includes Sichuan (277 species and 40 endemics), Yunnan (256 species and 43 endemics), and adjacent parts of Guizhou and Xizang A. R. The largest Umbelliferae genera in Chinese flora are Angelica (49 species), Bupleurum (47), Pimpinella (46), Seseli (39), Hymenidium (37), Heracleum (31), Ferula (27), Pternopetalum (21), and Acronema (21). There are 8 endemic genera of the family in China - Sinolimprichtia, Cyclorhiza, Chaerophyllopsis, Harrysmithia, Sinodielsia, Dickinsia, Melanosciadium, and Changium. Grounded on long-term examination of Chinese and especially foreign herbarium collections and available electronic resources, the checklist includes a new generic arrangement of many species, based on morphological and molecular analyses, revised synonymy, typification and distribution of species, with regard to Chinese provinces and autonomous regions. For 309 accepted specific and infraspecific names and synonyms, lectotypification had been proposed for the first time. 13 new nomenclarural combinations in the genera Kitagawia Pimenov, Ligusticopsis Leute, Oreocomopsis Pimenov \& Kljuykov, Seseli L., and Stenocoelium Ledeb. have been proposed.

\section{Обновленный конспект зонтичных (Umbelliferae) Китая: номенклатура, синонимия, типификация, распространение}

\author{
М. Г. Пименов
}

Ботанический сад Московского государственного университета им. М. В. Ломоносова, Воробьевы Горы, Москва, 117234, Россия

Ключевые слова: Арiaceae, зонтичные, Китай, Восточная Азия, систематика, таксономия, фитогеография, эндемичные виды, новые комбинации и синонимы, лектотипификация.

Аннотация. С 657 видами и 44 внутривидовыми таксонами, относящимися к 110 родам Umbelliferae, Китай обладает наивысшим таксономическим разнообразием зонтичных на видовом уровне среди всех стран мира. Число эндемичных видов также значительно (355). Главный центр разнообразия Umbelliferae в Китае находится в юго-западной части страны, где известно 357 видов, включая 126 эндемичных. Этот центр включает Сычуань (277 видов, 40 эндемичных), Юньнань (256 видов, 43 эндемичных) и прилегающие части Гуйджоу и Тибетского автономного района (Сизан). К числу крупнейших родов зонтичных Китая относятся Angelica (49 видов), Bupleurum (47), Pimpinella (46), Seseli (39), Hymenidium (37), Heracleum (31), Ferula (27), Pternopetalum (21) и Acronema (21). 9 родов зонтичных эндемичны для Китая (Sinolimprichtia, Cyclorhiza, Chaerophyllopsis, Harrysmithia, Sinodielsia, Dickinsia, Melanosciadium и Changium). На основании многолетних исследований гербарных коллекций, хранящихся в Китае и особенно за границей, а также доступных электронных ресурсов составлен конспект, включающий пересмотр родовой принадлежности многих видов, 
основанный на морфологических и молекулярных данных, пересмотренную синонимию, типификацию и распространение видов в соответствии с административным подразделением Китая. Для 309 принятых названий и синонимов видов и внутривидовых таксонов впервые предложена лектотипификация. Предложено 13 новых номенклатурных комбинаций в родах Kitagawia Pimenov, Ligusticopsis Leute, Oreocomopsis Pimenov \& Kljuykov, Seseli L. и Stenocoelium Ledeb.

\section{Introduction}

The checklist is composed of materials on systematics and phytogeography of Asian Umbelliferae stored in a special computer databases GNOM and ASIUM (Pimenov, Leonov, 1993, 2002, 2004), at the Botanical Garden of Moscow State University (original software by M. V. Leonov).

Among all countries of Asia and, most likely, the whole world, China boasts the greatest specific diversity in the Umbelliferae (Apiaceae). As of today, accumulated in the database ASIUM, 657 species from 110 genera of the family, are distributed in China, including Taiwan. The second place is held by Turkey, with 491 species and 112 genera, i.e. three fourth of those in China, although in considerably smaller territory. The number of endemic Umbelliferae species is also in China maximal per country (355 against 169 in Turkey).

\section{General notes on Chinese Umbelliferae and brief history of their investigation}

The publication of the English-language and updated version of the "Flora Reipublicae Popularis Sinicae" Umbelliferae treatment (Shan Renhwa et Sheh Menglan (eds.), 1979, 1985, 1992 under the name of "Flora of China" (Apiaceae/Umbelliferae volume 14 (Wu Zhengyi, Raven, Hong Dayuan, 2005, text; 2006, illustrations) was a considerable event in Eastern Asian and global floristic and systematic botany. Firstly, the achievements of Chinese botanists became available to the full extent to the botanical community outside China. The Chinese flora is very rich (no less than 30000 species) and for many chiefly temperate plant taxa is of a key value, as SW China and adjacent territories frequently regarded as a place of temperate flora differentiation in the North hemisphere (Takhtajan, 1957, 1969, 1970, 1978 [1988]).

"Flora of China" is an international project, organized by the Academia Sinica (Co-Chair - Prof. $\mathrm{Wu}$ Zhengyi) and the Missouri Botanical Garden, USA (Co-Chair - Prof. Peter H. Raven), with the help of experts from other countries. I was invited as a Western consultant on the Apiaceae/Umbelliferae, and therefore visited China three times, including a wonderful field trip to Yunnan and Sichuan led by Prof. Hu Zhihao from the Kunming university.
There were also consultations in Nanjing (then a recognized research center for the Umbelliferae in China) with leading Chinese experts in the Umbelliferae, pupils of Prof. Shan Renhwa, including Profs. Sheh Menglan and Pan Zehui, Drs. Yuan Changchi and Liu Qixin, as well as an intensive herbarium material checking in PE, NAS, KUN, SZ, CDBI and XJBI.

In accordance with principal conditions of the project, all controversies were to be discussed by Chinese and foreign contributors, and if there was no consensus, the Chinese treatment was to be adopted. Our Chinese colleagues were on a rather conservative position, seeking to preserve generic and specific concepts of "Flora Reipublicae Popularis Sinicae", as a result of previous development, what seemed to be only partly explicable. There were already carpological and molecular data to be added so as to upgrade the manuscript, especially the issues of generic delimitation. Additionally some specific names, adopted in Chinese scientific literature, appeared to be later synonyms of names of species, described previously and kept in foreign herbaria. It should be noted, that during the project "Flora Reipublicae Popularis Sinicae" Chinese botanists had rather limited access to Western herbaria and botanical libraries, including the most valuable ones, in our case those in P. We had an opportunity to publish our alternative critical revisions as well as new floristic data on some local or mainly local taxa in various periodicals, including Chinese ones ("Acta Phytotaxonomica Sinica", now "Journal of Systematics and Evolution"). In the final text of the Apiaceae/Umbelliferae treatment, these taxonomies were almost completely rejected; with the exception of Seseli revision. The latter one contained two alternative versions, with or without the species traditionally treated in Libanotis. Sometimes our viewpoints were implicitly expressed, for example, "New data show that the species A could be attributed to the genus N".

Outside of China I studied Chinese Umbelliferae, including type materials in various big herbaria, as P, LE, E, K, BM, W, WU, BP, G, FI, B, UPS, $\mathrm{GB}, \mathrm{LD}, \mathrm{O}, \mathrm{TI}, \mathrm{KYO}$, US, and some other. They contain a lot of species, described in the time, when Chinese botany was lagging behind. In this period, collections made by foreigners, now kept 
in the European, American and Japanese herbaria, were the first evidence of richness of Chinese flora. Chinese plants were collected by travelers, missionaries, special expeditions, diplomats, agents of horticultural companies, etc., who worked all over world. In China, however, catholic missionaries, first from France, then from other countries (Germany, Italy and Ireland) were of primary importance. Their activity and sometimes tragic fate is described in detail in a very interesting book "The fathers of botany" (Kilpatrick, 2014). Now Umbelliferae gatherings, mainly treated by a Paris botanist Adrien Franchet $(1882,1883,1885,1888,1894,1895)$, are kept perfectly safe, side by side with the collections of plants from other families, in $\mathrm{P}$ - Muséum National d'Histoire Naturelle (Phanerogamie) in Paris.

The situation with Chinese and other Asiatic Umbelliferae collected by various plant hunters is totally different. Those specimens, which were concentrated in Berlin-Dahlem (B) thanks to (Karl Friedrich August) Hermann Wolff, the leading German Umbelliferae monographer of the 20-30s of the XX century, the author of three large treatments of the family for Adolf Engler's "Das Pflanzenreich" (Wolff, 1910, 1913, 1927) and numerous descriptions of new species (Wolff, 1924a, b, 1925a-c, 1926), for instance in a series "Umbelliferae asiaticae novae relictae" (Wolff, 1929a, b, 1930a, b, 1934), were partly lost. A considerable amount of type material in Berlin-Dahlem was burnt or destroyed during World War II. The duplicates of some were later found in other herbaria. So, these species remain to be incertae sedis, although their names are not illegitimate.

Among British collections, vast materials by Georg Forrest in E and, of course, the Umbelliferae types in $\mathrm{K}$, are of particular interest. They include the species firstly distinguished in Flora of British India (Clarke, 1879) and distributed also in the adjacent parts of China.

For NW and partly NE China, Russian collections, for instance, those delivered by the big expeditions of the XIX century (N. Przhevalsky's, G. Potanin's, and etc.), earlier gatherings made by Russian diplomats near Peking, and materials related to Komarov's Flora of Manchuria (Komarov, 1907), are of a priority value. Typification for Russian Central Asian and East Asian collections of the Umbelliferae kept in LE, was made by Vinogradova (2004, 2010). For NE China these data were added in the first half of the 20th century from Japanese herbarium materials (Kitagawa, 1960, 1979).
American collectors working for horticultural companies appeared in China somewhat later, however they were quite active in herbarisation of local plants. Among them, the collections of F. Kingdon-Ward, F. Forbes and J. Rock were the most valuable. American herbaria have also numerous specimens, duplicating (not infrequently, isotypes or isolectotypes) of those kept in European herbaria.

The Asiatic Umbelliferae, and Chinese in particular, are strong meat for modern taxonomists for several reasons. First of all, the Umbelliferae species and genera are very numerous in China. Second, many specialists from different countries studied them independently and with regard to their own taxonomic tradition; therefore their results have not been coordinated up to now. Many species, especially rare and narrow endemics, required further research. Carpological descriptions, essential for Umbelliferae taxonomy, are often superficial, if any. Many key collections, for instance, those that formed the basis for Hermann Wolff's descriptions, have been lost. Molecular data that are of increasing importance for modern taxonomic botany include less than half Chinese species, and many critical genera are not studied. Quite predictably, there a broad range of opinions on some species exists their generic attribution, volume, synonymy, distribution, etc.

Now IT resources, such as the JSTOR Global Plants database, provide new opportunities to compare the numerous images of type or presumable type materials from various herbaria and to correct the qualification of type specimens. This is a valuable addition, especially productive given a specialized computer database, similar to our ASIUM. Unfortunately, up to now there is no data on the Umbelliferae from Chinese herbaria in Global Plants on JSTOR.

\section{Conspectus of Chinese Umbelliferae}

The checklist includes 657 accepted names of Chinese Umbelliferae, according to our current taxonomy, although some names of validly described species are to be attributed now to nomina incertae sedis. First, it is some taxa, described or published in the 20-30s of the XX century by Hermann Wolff. Among them there are some species classified by Wolff as Ligusticum and Peucedanum species. Following Leute (1969), most Chinese Ligusticum species were transferred to Ligusticopsis Leute, a satellite genus, but such transfers would not be possible for L. kiangsiense $\mathrm{H}$. Wolff, L. kulingense 
H. Wolff, and L. littledalei Fedde ex H. Wolff without investigation of type materials. The latter have not been found thus far. A similar or maybe even more complicated situation is with Wolff's Chinese Peucedanum species. Wolff described $P$. dielsianum Fedde ex Y.Wolff, $P$. diversifolium $\mathrm{H}$. Wolff, and P. yunnanense $\mathrm{H}$. Wolff, whose type materials have been vanished. The majority of Chinese and other East Asian species of Peucedanum are not true Peucedanum taxa, as they are unrelated to the type species, $P$. officinale L., and its closest allies.

The hope to decipher these species still remain, however. In G, and later in W, I (Pimenov, 1998) found the isotypes of Peucedanum wolffianum Fedde ex H. Wolff from Saghalien, and the species was easily identified as Conioselinum chinense (L.) Britton, Sterns et Poggenb. Recently I found in Oslo (O) 16 sheets of type collections by Arthur von Rosthorn from China; the species were described by L. Diels from Berlin-Dahlem, but most probably were lost in B and most probably kept now only in Oslo University herbarium.

The Umbelliferae taxa are arranged in the checklist in the alphabetical order of accepted names. The abbreviations or full transcription of the authorities of species and genera comply with the international standard - "The authors of plant names" by Brummitt and Powell (1992), with the only exception, in this context most essential. It deals with Chinese botanists' names. Following Xu Zhaoren and Nikolson (1992), I believe the abbreviation of names of Chinese authors of plant names, in the Western style (initials and surname) to be inconvenient, as Chinese family names are low variable (in the country there are millions of namesakes, and numerous ones among botanists), in contrast with given names, being diverse and numerous. So, Chinese personal names are used here in their full form as more informative, both in taxa authorities and in the reference list. Authors of Chinese plant names are written here in pinyin (pīnyinn), the official romanization system for Standard Chinese.

I tried to find type material for all plant names used in the contribution, but it seems to be only partly feasible. Sometimes I was forced to refer to type locality(ies) cited in the protologue; the absence of herbarium abbreviation (according to Index Herbariorum, Thier.) means that the locality of type material was not indicated in the protologue and now is unknown.

The distribution of each species and infraspecific taxon in China is indicated with regards to provinces and autonomous regions of the country, which are presented here as main geographical divisions North-Western (NW), North (N), North-Eastern (NE), Tibet, Central (Centr.), South-Western (SW), and Southern (S). These parts could be regarded, of course, only very roughly as natural regions.

Acronema alpinum Liou Shoulu et Shan Renhwa, 1980, Acta Phytotax. Sin. 18(2): 200, tab. 4, fig. 3-7.

Type: CHINA. "Xizang, Gyaca, 4750-4800 m, 19.08.1975, Qinghai-Xizang Exped. 751424" (holotype HNWP; isotype KUN!).

Distribution in China: Tibet: Xizang A. R., Qinghai. Endemic.

Acronema astrantiifolium H. Wolff, 1929, Repert. Spec. Nov. Regni Veg. 27: 192.

Distribution in China: S: Taiwan. Endemic.

Angelica morrisonicola Hayata var. nanhutashanensis Liu Tangshui, Chao Chuangying et Chuang Tsaniang, 1961, Quart. J. Taiwan Mus. 14(12): 21.

Type: CHINA. "[Taiwan], Nanhutashan, Ilan Hsien, 22. 07. 1921, Sasaki" (holotype TAI).

Distribution in China: S: Taiwan.

Angelica multicaulis Pimenov, 1972, Byull. Moskovsk. Obšč. Isp. Prir. Otd. Biol. 77(5): 90, tab. 1, 3, map 2(2).

Type: KIRGHIZIA. "Asia Media, Tian-Shan centralis, jugum Terskei-Alatau, in valle fluvii Sarydzhas inter ostia fluminis Kuilu et M. Taldysu, ad declivia conglomeratis, 21. 08. 1969, Pimenov, Borjaev, Trusov 2095" (holotype LE!; isotypes LE!, MW!).

= Angelica tichomirovii V. M. Vinogr., 1986, Novosti Sist. Vyssh. Rast. 23: 93, fig. 1.

Type: CHINA. "Dzhungaria, Tian-Schan, jugum Iren-Chabirga, mons Monguto, 2700 m s.m. 02. 08. 1879, Regel" (holotype LE!).

Distribution in China: NW: Xinjiang Weiwuer A. R.

Distribution outside China: Kirghizia.

Angelica nakaiana (Kitag.) Pimenov, 1999, Feddes Repert. 110(7-8): 482.

= Homopterix nakaiana Kitag., 1937, Bot. Mag. (Tokyo) 51: 809, fig. 2.

$\equiv$ Coelopleurum nakaianum (Kitag.) Kitag., 1968, J. Jap. Bot. 43(10-11): 427.

Type: KOREA. "Prov. Kan-Nan: In herbidis in summa montis Sya-zitu-hô, $2500 \mathrm{~m}$ alt., 10. 
08. 1935, Nakai 15610" (lectotype TI!, designated here); ibid. "07. 1931, Kisinami” (syntype).

Distribution in China: NE: Jilin.

Distribution outside China: Korea.

Angelica nitida H. Wolff, 1926, Acta Horti Gothob. 2: 317.

Type: CHINA. "Sze-ch'uan bor.: Huangch'en-kuan, in silva mixta sparsa herbosa?, ca. 3300 m, 19. 08. 1922, H. Smith 4069" (lectotype UPS!, designated here; isolectotypes GB!, barcode GB0048796, PE!, W!).

= Angelica wulsiniana H. Wolff, 1930, Repert. Spec. Nov. Regni Veg. 27: 334.

Type: CHINA. 'Kansu: Kar Ching K'ou, near Old Taochow, altitude 3100 to $3400 \mathrm{~m}, 27.08 .1923$, R. C. Ching 848 " (holotype US!).

$=$ Angelica chinghaiensis Shan Renhwa ex Fu Kuntsun, 1981, Fl. Tsinling. 1(3): 421, 462.

Type: CHINA. "Tsinghai: Menyang Hsien, Sien-mi-sze, alt. 2450 m, 06. 09. 1936, Liou KiMou 7006" (holotype WUK).

$=$ Angelica songpanensis Shan Renhwa et $\mathrm{Pu}$ Fating [1993, Vasc. P1. Hengduan Mount. 1: 1344, nom. inval.] 1995, Acta Phytotax. Sin. 33(5): 480, fig. 3.

Type: CHINA. "Sichuan, Songpan, Huanglong, Xue-bao-ding Mt., alt. $4000 \mathrm{~m}$, in subalpine forests, 14. 10. 1983, Pu Fating et al. 159" (holotype CDBI!)

Distribution in China: NW: Gansu; Tibet: Qinghai; SW: Sichuan. Endemic.

Angelica nubigena (C. B. Clarke) P. K. Mukh., 1983, Bull. Bot. Surv. India 24(1-4): 42.

$\equiv$ Heracleum nubigenum C. B. Clarke, 1879 , in Hooker f.(ed.), Fl. Brit. India 2: 42.

Type: INDIA. "Sikkim, alt. 10000-14000 ft., Chola Pass, 06. 11. 1849, Hooker" (lectotype K!, designated by L. Constance in $\mathrm{K}$ (Watson, 1998: 372); isolectotypes CAL, E!, G!, K!); "Yakla Pass [Sikkim], 14000', 19. 10. 1865, Clarke 9783" (syntype E!).

Distribution in China: Tibet: Xizang A. R.

Distribution outside China: India.

Angelica paeoniifolia Shan Renhwa et Yuan Changchi, 1980, Acta Phytotax. Sin. 18(3): 378.

Type: CHINA. "Xizang (Tibet): Nuchiang, Biru Xian, alt. 3800-4000 m, 12. 09. 1976, D. D. Tao 11320 " (holotype PE; isotype KUN!).

Distribution in China: Tibet: Xizang A. R. Endemic.
Angelica pinnatiloba Shan Renhwa et Pu Fating [1993, Vasc. Pl. Hengduan Mount. 1: 1345, nom. inval.], 1995, Acta Phytotax. Sin. 33(5): 481, fig. 3.

Type: CHINA. "Sichuan, Songpan, Rawu distr., Equigou, alt. 2700 m., at the edge or by streams, 19. 10. 1983, Pu Fating et al. 197" (holotype CDBI!).

Distribution in China: China (SW: Sichuan). Endemic.

Angelica polymorpha Maxim., 1873, Bull. Acad. Imp. Sci. Saint-Petersbourg 19: 185.

$\equiv$ Rompelia polymorpha (Maxim.) Koso-Pol., Bull. Soc. Imp. Naturalistes Moscou, s.n. 29: 125. 1916.

Type: JAPAN. "In Nippon mediae jugo Hakone [Honshu] silvis frondosis vetustis ad rivulos, ediam in silvis acerosis, 18. 10. 1862, Tschonoski [Sugawa]" (syntypes K!, barcode K001097187, LE!); "In Kiusiu inferiore, Nagasaki, jugo Kundshosan, in silvis mixtis udis, 10. 1863, Maximowicz" (lectotype LE!, designated by Grubov (Vinogradova, 2004: 27); isolectotypes G!, barcode G00359376, GH, NY, P!, barcode P00752989).

= Angelica fallax H.Boissieu, Bull. Soc. Bot. France 59: 199. 1912.

= Angelica polymorpha Maxim. var. fallax (H.Boissieu) Kitag. Neo-Linneam. Fl. Manshur.: 474. 1979.

Type: KOREA. "Quelpaert (ile au Sud de la Corée), près de Hongno, vers les cascades, 0207. 1907, Faurie 1825" (lectotype P!, barcode P00602145, designated here; isolectotypes G!, barcode G00359369, KYO!); "Quelpaert, 08. 04. 1908, Taquet 4649" (syntype); "Rivage de Sysken, 25. 06. 1908, Taquet" (syntype P!); "[Quelpaert] sur le ravage, 08. 04. 1911, Taquet 4796" (syntypes G!, barcode G00359370, P!, barcode P03237838).

= Peucedanum taquetii H.Wolff, Repert. Spec. Nov. Regni Veg. 21: 245. 1925.

Type: KOREA. "Corea: Honguo, secus torrents, 15. 11. 1907, Taquet 109" (lectotype G!, barcode G00359356, designated here; isolectotype E!, barcode E00417562).

= Angelica sinuata H.Wolff, Repert.Spec. Nov. Regni Veg. 27: 333. 1930.

Type: CHINA. "Tschili: Hsiao-wu-tai-shan, Yang kia ping, Peiko, in valle umbroso, $1050 \mathrm{~m}$ s.m., 26. 08. 1921, H. Smith 322" (lectotype UPS!, designated here; isolectotypes PE!, S!, barcode S-G 420); "Schan-tung: Kiautschou, Cap Yatau, in convallibus et in declivibus, in graminosis frequens, Forstamt Tsingt 636" (syntype); "Schan-tung: 
Lauschan-Gebirge, 350 m s.m., Krug, Forstamt Tsingt 387" (syntype).

= Angelica wolffiana Fedde ex H. Wolff, 1930, Repert. Spec. Nov. Regni Veg. 28: 110.

$=$ Conioselinum wolffianum (Fedde ex Wolff)

Nakai, 1952, Bull. Natl. Sci. Mus. 31 (Syn. Sk. Kor. Fl.): 87.

Type: KOREA. "Korea.Taquet $860 "$.

Distribution in China: N: Shaanxi, Hebei, Shandong; NE: Heilongiiang, Jilin, Liaoning; Centr.: Henan, Jiangsu, Anhui, Hubei, Zhejiang; SW: Guizhou.

Distribution outside China: Japan, Korea.

Angelica porphyrocaulis Nakai et Kitag., 1934. Rep. First Sci. Exped. Manchoukuo (sect. IV) 1: 33, tab. 10.

Type: CHINA. "Jehol, in monte Wu-ling-shan, 02 IX 1933, Nakai, Honda, Kitagawa" (holotype TI!).

Distribution in China: NE: Jilin. Endemic.

Angelica porphyrocaulis Nakai et Kitag. f. latisecta Kitag., 1936, J. Jap. Bot. 12(4-5): 313.

Type: CHINA. "Manshuria: prov. Je-he: in monte prope Hsing-Iing, 31. 08. 1933, Nakai, Honda, Kitagawa".

Distribution in China: NE: Jilin.

Angelica pseudoselinum H. Boissieu, 1903, Bull. Herb. Boissier (2 sér.) 3(10): 848.

Type: CHINA. "Pelouses Heou-Pin près de Tchen-Keou, alt. 2000 m, 16. 07. 1893, Farges 1264" (lectotype P!, barcode P00602147, designated here; isolectotypes K!, P!, barcodes P00602146, P00602148, P00602149).

Distribution in China: Centr.: Hubei; SW: Sichuan. Endemic.

Angelica saxatilis Turcz. [Bull. Soc. Imp. Naturalistes Moscou 11 N 536. 1838, nom. inval. (Art. 39.1] ex Ledeb., 1844, Fl. Ross. 2: 296.

$\equiv$ Physolophium saxatile Turcz., 1844, Bull. Soc. Imp. Naturalistes Moscou 17: 728.

$\equiv$ Coelopleurum saxatile (Turcz. ex Ledeb.) Drude, 1898, in Engler \& Prantl (eds.), Nat. Pflanzenfam. 3(8): 213.

$\equiv$ Angelica gmelinii (DC.) Pimenov var. saxatilis (Turcz. ex Ledeb.) Vorosch., 1985, in Skvortzov (ed.), Florist. issl. v razl. raionakh SSSR: 184.

Type: RUSSIA. "In Sibiriae transbaicalensis subalpinis inter Bargusin et Angara super inque Davuriae rupibus ad fl. Schilka [In praeruptis lapidosis ad fl. Schilka prope rivulum Serebrianka], 1833, Turczaninow" (lectotype LE!, designated by Vinogradova, 2005: 959; isolectotypes G!, barcode G00359379, H!, barcode H1014089, P!, barcode P02573456); "Schilka, 1833, Turczaninow" (syntypes LE!).

= Angelica maculata Turcz., 1840, Bull. Soc. Imp. Naturalistes Moscou 13: 72.

Type: RUSSIA. "Ad viam Ochotensem et in insulis Kurilensibus [Prope Ochotiam], Kuznetzow" (lectotype LE!, designated here).

= Physolophium saxatile Turcz. var. angustifolia Fr.Schmidt ex Maxim., 1859, Mém. Acad. Imp. Sci. Saint-Petersbourg Divers Savants 9 (Prim. Fl. Amur.): 126.

$=$ Angelica lucida L. var. angustifolia (Fr. Schmidt ex Maxim.) Weinert, 1973, Feddes Repert. 84(4): 307.

= Coelopleurum alpinum Kitag., 1941, Rep. Inst. Sci. Res. Manchoukuo 5: 146, 156.

Type: CHINA. "Manshuria: Prov. T'ung-hua, in declivitate umbroso alpino in Mt. Chang-pai-shan (Paiktusan), ca. $2000 \mathrm{~m}$ alt., 29. 07. 1940, Kitagawa" (holotype TI!).

- Coelopleurum gmelinii auct. non Ledeb.: Popov, 1957, Fl. Srednei Sibiri 1: 478.

Distribution in China: NE: Heilongjiang, Jilin.

Distribution outside China: Russia, Korea, Mongolia.

Angelica setchuenensis Diels, 1900, Bot. Jahrb. Syst. 29 (3-4) (Fl. Centr. China): 500.

Type: CHINA. "Suchuan S: Kinshan: Urwald bei Shin Tzu-k'ou, von Rosthorn 86" (syntype O); "Suchuan S: Waldgang bei Chue ch'ang-wan, von Rosthorn 1048" (syntype O).

= Angelica valida Diels, 1900, Bot. Jahrb. Syst. 29 (3-4) (Fl. Centr. China): 501.

Type: CHINA. "Setchuen. Nan ch'uen: Ch'ang ling kang, 28. 08. 1891, von Rosthorn 645" (lectotype O!, designated here); "Setchuan S: Ket ou p'ing, Birgwald, von Rosthorn 1097" (syntype B - destr.?); "Setshuan S: Yachih-pa, von Rosthorn 476" (syntype B-destr.?).

Distribution in China: Centr.: Hubei; SW: Sichuan. Endemic.

Angelica sinensis (Oliv.) Diels, 1900, Bot. Jahrb. Syst. 29 (3-4) (Fl. Centr. China): 500.

$\equiv$ Angelica polymorpha Maxim. var. sinensis Oliv., 1891, in Hook. f. Icon. pl. (ser. 3) 20: tab. 1999.

Type: CHINA. "Sichuan, Kangding, 1885-1888, Henry 7143” (syntypes K, barcode K001097186, 
NY!, barcode NY00405716); "Wushan, Henry 6897" (lectotype P!, barcode P03237864, designated here; isolectotypes E!, barcode E00265828, G!, K!, barcode K001097184, P!).

Distribution in China: NW: Gansu; N: Shaanxi; NE: Heilongjiang; Centr.: Henan, Anhui, Hubei; SW: Sichuan, Guizhou, Yunnan; S: Guangxi Zhuang A. R., Guangdong. Endemic.

Angelica smithii H. Wolff, 1926, Acta Horti Gothob. 2: 320.

Type: CHINA. "Chili: Hsiao-wu-tai-shan, Yangkia-p'ing, Pei-ko, am Rande eines Laubwaldes, 12. 09. 1921, H. Smith 697" (lectotype UPS!, designated here; isolectotype GB!, barcode GB0048800).

= Angelica urticifoliata H.Wolff, 1926, Acta Horti Gothob. 2: 320.

Type: CHINA. "Chili: Hsiao-wu-tai-shan, Yangkia-p'ing, Pei-ko, 12. 09. 1921, H. Smith 706" (holotype GB!, barcode GB0048801).

Distribution in China: NE: Jilin. Endemic.

Angelica sylvestris L., 1753, Sp. pl.: 251.

$\equiv$ Athamanta sylvestris (L.) Weber ex F.H.Wigg, 1780, Prim. Fl. Holsat.: 26.

$\equiv$ Selinum angelica Roth., 1788, Tent. Fl. Germ. 1: 133 .

$\equiv$ Peucedanum angelica Caruel, 1889, in Parlatore, Fl. Ital. 8: 281.

$\equiv$ Selinum agriangelica E. H. L. Krause, 1904, in Sturm (ed.), Deutschl. Fl.(ed. 2) 12: 122, fig. 25.

Type: EUROPE. "In Europae frigidioris subhumidis sylvaticis, Herb. Clifford, 97, Angelica" (lectotype BM-Cliff!, designated by Reduron, Jarvis, 1992: 557; see also: Reduron, 2007).

$=$ Taeniopetalum peucedanoides Bunge, 1851, Beitr. Fl. Russl. (Al. Lehmann Reliq. Bot.): 127.

Type: EUROPE, RUSSIA. "Auf der Linie zwischen Orenburg und Orskaja, 07. 1840, Lehmann 540" (syntypes P!, W!); "Zwischen Ufa und Sterlitamak, 22. 08. 1840, Lehmann" (lectotype $\mathrm{P}$ !, designated here).

Distribution in China: NW: Xinjiang Weiwuer A. R.

Distribution outside China: Russia, Mongolia, Kazakhstan, Georgia, Turkey, Europe, Africa, N. America.

Angelica tarokoensis Hayata, 1921, Icon. P1. Formosan. 10: 27.

Type: CHINA. "Taiwan, Naitailuko, Hwalien Hsien [Inter Batakan et Naitaroko], 08. 1917, Hayata, Sasaki" (lectotype TI!, designated here; isolectotype TAIF).
Distribution in China: S: Taiwan. Endemic.

Angelica ternata Regel et Schmalh., 1878, Trudy Imp. S.-Peterburgsk. Bot. Sada 5(2): 590, tornata; 1881, Descr. Pl. Nov. Rar. Fedtsch.: 32.

$\equiv$ Callisace ternata (Regel et Schmalh.) KosoPol., 1916, Bull. Soc. Imp. Naturalistes Moscou, s.n. 29: 179.

Type: KIRGHIZIA. "In Kokania, a trajectum Isfairam ad Alai, 8000', 20. 07. 1871, O. A. Fedtschenko" (lectotype LE!, designated by Vinogradova, 1999: 81).

$=$ Peucedanum skardicum C. B. Clarke, 1879, in Hooker f. (ed.), Fl. Brit. India 2: 711.

Type: PAKISTAN. "Baltistan, near Scardo, alt. $12000 \mathrm{ft}$ [Birjila, Kashmir, 12000 ft.], 01. 08. 1876, Clarke 29882A" (holotype K!).

= Angelica strattoniana Aitch. et Hemsl., 1882, J. Linn. Soc. Bot. 19: 164, tab. 13, fig. 1-7.

Type: AFGHANISTAN. "Nuristan, Mount Sikaram westwards to Mount Seratigah, growing outside the clefts of rocks from 9000 to 13000 feet, 26. 07 . 1880, Aitchison 394" (lectotype K!, designated here by Pimenov and Jacquemoud; isolectotypes DD!, G-BOIS!).

Distribution in China: NW: Xinjiang Weiwuer A. R.

Distribution outside China: Pakistan, Kirghizia, Tajikistan, Uzbekistan, Afghanistan.

Angelica tianmuensis Pan Zehui et Zhuang Tide, 1995, Acta Phytotax. Sin. 33(1): 86, fig. 1.

Type: CHINA. "Zhejiang: Tianmu Mts., Daogualianhua, alt. $1100 \mathrm{~m}$, under forests, 28. 10. 1990, Lin Xintian et al. 90116" (holotype NAS!).

Distribution in China: Centr.: Zhejiang. Endemic.

Angelica wilsonii H. Wolff, 1930, Repert.Spec. Nov. Regni Veg. 27: 335.

$\equiv$ Angelica sinensis (Oliv.) Diels var. wilsonii (H. Wolff) Pan Zehui et M. F. Watson, 2004, Acta Phytotax. Sin. 42(6): 562.

Type: CHINA. "West Szechuan, Tan-kuei, [Long] Hsien 08.1907, Wilson 201" (lectotype GH!, barcode GH00075074, designated here; isolectotype K!, barcode K001097109).

$=$ Angelica omeiensis Yuan Changchi et Shan Renhwa, 1985, Bull. Nanjing Bot. Gard. 1983: 6, fig. 2.

Type: CHINA. "Sichuan: Xixiangchi, Omei Mons, Omei Xian, alt. 2100 m, 31. 07. 1977, Yue JinSan, Y. F. Dong 7701" (holotype NAS!). 
Distribution in China: SW: Sichuan, Yunnan. Endemic.

Anthriscus sylvestris (L.) Hoffm., 1814, Gen. p1. Umbell.: 40, 46, tab. 1, fig. 19.

$\equiv$ Chaerophyllum sylvestre L., 1753, Sp. pl.: 258.

$\equiv$ Chaerophyllum infestum Salisb., 1796, Prodr. stirp. Chap. Allerton: 167.

$\equiv$ Myrrhis sylvestris (L.) Spreng., 1813, Neue Schrften Naturf. Ges. Halle 2, 1 (Pl. Umb. Prodr.): 29.

$\equiv$ Cerefolium sylvestre (L.) Besser, 1809, Prim.

fl. Galic. 1: 218.

$\equiv$ Myrrhoides sylvestre (L.) Kuntze, 1891, Revis. Gen. pl. 1: 268.

$\equiv$ Selinum cicutaria E. H. L. Krause, 1904, in Sturm, Deutschl. Fl. (ed. 2) 12: 71.

$\equiv$ Chaerefolium sylvestre (L.) Schinz et Thell., 1908, Vierteljahrsschr. Naturf. Ges. Zürich 53: 554.

三 Scandix sylvestre (L.) Koso-Pol., 1916, Bull.

Soc. Imp. Naturalistes Moscou, s.n. 29: 505.

Type: EUROPE. "In Europae pomariis et cultis, 365.1" (lectotype LINN, designated by I. C. Hedge, J. Lamond, 1987: 85; see also: Reduron, 2007).

= Chaerophyllum nemorosum M. Bieb., 1808,

Fl. Taur.-Caucas. 1: 232.

= Anthriscus nemorosa (M. Bieb.) Spreng., 1813, Neue Schriften Naturf. Ges. Halle 2(1) (P1. Umb. Prodr.): 27.

= Myrrhoides nemorosum (M. Bieb.) Kuntze, 1891, Revis. Gen. Pl. 1: 268.

= Anthriscus sylvestris (L.) Hoffm. subsp. nemorosa (M. Bieb.) Koso-Pol., 1920, Izv., Glavn. Bot. Sada RSFSR 36, 1 (Fl. As. Ross. 15): 103.

$=$ Chaerefolium sylvestre (L.) Schinz et Thell. subsp. nemorosum (M. Bieb.) Thell., 1926, in Hegi, Ill. Fl. Mitt.-Eur. 5, 2: 1025.

= Chaerefolium nemorosum (M. Bieb.) Bornm., 1928, Repert. Spec. Nov. Regni Veg. 25: 281.

Type: RUSSIA. "In sylvis ad fluvium Terek sub arboribus, Marchall von Bieberstein" (lectotype LE!; designated here; isolectotypes B-Willd!, LINN-Sm!).

$=$ Anthriscus anatolica Boiss., 1844, Ann. Sci. Nat. Paris (sér. 3, Bot.) 2 (Pl. Aucher.): 59.

= Anthriscus nemorosa (M. Bieb.) Spreng. var. anatolica Boiss., 1872, Fl. Orient. 2: 911.

Type: TURKEY. "In glareosis regionis alpinae, in declivibus summis Olympi Bithini ad septentrionem versis supra vallem Kirkbounar, in monte Gargaro, Aucher-Eloy 3614" (syntypes G-BOIS!, K!); "Olympi Bithyni, 07. 1842, Boissier" (lectotype G-BOIS!, designated by Spalik, 1995: 79; isolectotypes $\mathrm{K}$ !, MW!, W).
= Anthriscus macrocarpa Boiss. et Heldr., 1856, Diagn. Pl. Orient. (ser. 2) 2: 100.

= Anthriscus nemorosa (M. Bieb.) Spreng. var. macrocarpa (Boiss.) Boiss., 1872, Fl. Orient. 2: 911.

Type: TURKEY. "Tmoli in Lydiâ [Tmoli umbrosis supra Philadelphiam], Boissier" (lectotype G-BOIS!, designated by Spalik, 1995: 84); "in umbrosis montium Anatoliae, Cariae, Pinard" (syntype); "Lyciae in monte Solyma, Heldreich 2098" (syntype G). GREECE. "In montibus Graeciae Olenos, Heldreich 598" (syntypes BM!, E, G!, H!, WAG); "Kyllene, 1852, Orphanides 253" (syntypes E, G, WAG).

= Anthriscus nemorosa (M. Bieb.) Spreng. var. mollis Boiss., 1872, Fl. Orient. 2: 911.

Type: GEORGIA. "In montibus Daghestaniae 7000'-8000', Ruprecht" (syntype); "[In montibus] Georgiae Caucasicae, Hohenacker" (syntypes E, G, G-BOIS!). IRAN. "Persia in monte Demavend, Kotschy 376" (syntype G-BOIS!). TURKEY. "Armenia turcica ad Erzerum et Gumuschkhane, Calvert, Bourgeau" (syntype G-BOIS!); "Champs incultés bords des ravines près Gummus-Hhane, 03. 06. 1862, Bourgeau 95" (syntypes G-BOIS!, W); "[In montibus] Ponti Lazici supra Djimil, alt. 7500', 07-08. 1866, Balansa 121" (lectotype G-BOIS!, designated by Spalik, 1995: 78; isolectotypes K!, US).

= Anthriscus nemorosa (M. Bieb.) Spreng. var. glabra Boiss., 1872, Fl. Orient. 2: 911.

Type: TURKEY. "In regione alpinâ Armeniae australis ad Bakkermaadan, 05. 1852, Noë 1091" (syntype G-BOIS!); "In summo monte Bingöl dagh alt. 9800', 23. 08. 1859, Kotschy 387" (lectotype G-BOIS!, designated by Spalik, 1995: 84 ; isolectotype $\mathrm{P}$ ).

= Myrrhis chaerophylloides Hance, 1878, J. Bot., n.s. 7(16): 108 .

Type: CHINA. "In ins. Silver Island, juxta Chinkiang, 05. 1863, Hay" (syntype BM!); "Circas oppidum Chinkiang, 1876, Stronach 10127” (syntype).

$=$ Anthriscus velutina Sommier et Levier, 1895, Nuovo Giorn. Bot. Ital., ser. 2, 2: 73, "velutinum".

Type: RUSSIA. "In alto valle fluminis Tieberda, ditionis Kubanensis [Kuban - in valle Tieberda, in regione sylvatica], $1500 \mathrm{~m}, 31.08 .1890$, Sommier, Levier 537(48)" (lectotype FI!, designated here; isolectotypes FI!, G!).

= Anthriscus yunnanensis W. W. Sm., 1915, Notes Roy. Bot. Gard. Edinburgh 8: 331.

= Oreochorte yunnanensis (W. W. Sm.) KosoPol., 1916, Bull. Soc. Imp. Naturalistes Moscou, s.n. 29: 152. 
Type: CHINA. "Yunnan, eastern flank of the Lichiang Range, Lat $27^{\circ} 20$ N. Open stony pasture, 07. 1910, Forrest 6020" (syntypes E!, K!); "Yunnan, eastern flank of the Lichiang Range. Lat. 27030' N. Mountain pasture on the margin of pine forests, 11000 ft., 06. 1910, Forrest 5914" (lectotype E!, designated here; isolectotypes $\mathrm{K}$ !, $\mathrm{PE}$ !).

= Anthriscus sylvestris (L.) Hoffm. var. aemula Woronow, 1931, Trudy Bot. Sada Akad. Nauk URSS 43, 2 (Flora Rossiae Austro-Orient. 5): 770.

$=$ Anthriscus aemula (Woronow) Schischk., 1950, in Schischkin, Fl. URSS 16: 129.

= Anthriscus sylvestris (L.) Hoffm. subsp. aemula (Woronow) Kitam., 1962, Acta Phytotax. Geobot. 20: 195 .

Type: EUROPE, RUSSIA. "Bashkiria, canton Sterlitamak, prope pag. Togus-Temir, sylvae querceto-betuletae, 12. 06. 1928, Kucherovskaya 475" (lectotype LE!, designated by V. M. Vinogradova, 2003: 106).

= Anthriscus nemorosa (M. Bieb.) Spreng. var. hirtifructa Ohwi, 1953, Fl. Jap.: 843.

= Anthriscus sylvestris (L.) Hoffm. var. hirtifructa (Ohwi) H. Hara, 1954, Enum. Sperm. Jap. 3: 300.

= Anthriscus aemula (Woronow) Schischk. var. hirtifructa (Ohwi) Kitag., 1960, Bull. Natl. Sci. Mus. 5, 1 (46): 9.

= Anthriscus aemula (Woronow) Schischk. f. hirtifructa (Ohwi) Kitag., 1979, Neo-Linneam. Fl. Manshur.: 477.

Type: JAPAN. "Shikoku: Urbe Tokushima in Awa, Nakai 1313" (holotype NSM).

= Anthriscus nemorosa (M. Bieb.) Spreng. var. glabriuscula Nasir, 1957, Biologia (Lahore) 3(1): 61.

Type: PAKISTAN. "Kashmir, near Kamri bungalow, 9-10000 ft., 13. 07. 1946, Stewart, Nasir 22724" (holotype RAW; isotypes K!, UC).

Distribution in China: NW: Xinjiang Weiwuer A. R., Gansu; Tibet: Xizang A. R.,Qinghai; N: Neimenggu A. R., Shaanxi, Shanxi, Hebei, Shandong; NE: Heilongjiang, Jilin, Liaoning; Centr.: Henan, Jiangsu, Anhui, Hubei, Hunan, Jiangxi, Zhejiang; SW: Sichuan, Guizhou, Yunnan.

Distribution outside China: Russia, Japan, Korea, Nepal, India, Pakistan, Kazakhstan, Kirghizia, Uzbekistan, Turkmenistan, Afghanistan, Iran, Azerbaijan, Georgia, Armenia, Turkey, Iraq, Yemen, Lebanon, Syria. Europe, Africa, N. America.

Aphanopleura capillifolia (Regel et Schmalh.) Lipsky, 1896, Bull. Acad. Imp. Sci. SaintPetersbourg (ser. 5) 4(4): 379.

$\equiv$ Pimpinella capillifolia Regel et Schmalh., 1881, Izv. Obšč. Ljubit. Estestv. 34(2): 29. $\equiv$ Carum capillifolium (Regel et Schmalh.) Koso-Pol., 1916, Bull. Soc. Imp. Naturalistes Moscou, s.n. 29: 199, nom. illeg. (Art. 53.1), non Kar. et Kir. (1841).

Type: UZBEKISTAN. "In Turkestaniae deserto inter fluvium Syr-Darya et Taschkent, 19. 05. 1871, O. A. Fedtschenko" (lectotype LE!, designated by Geldykhanov, 1992: 131).

- Aphanopleura leptoclada auct. non Lipsky: Shan Renhwa, 1985, in Fl. Reipubl. Popularis Sin. 55, 2: 3 .

Distribution in China: NW: Xinjiang Weiwuer A. R.

Distribution outside China: Kazakhstan, Kirghizia, Tajikistan, Uzbekistan, Turkmenistan.

Apium graveolens L., 1753, Sp. pl.: 264.

三Seseli graveolens (L.) Scop., 1771, Fl. Carniol. (ed. 2) 1: 215.

$\equiv$ Apium celleri Gaertn., 1788, Fr. Sem. Pl. 1: tab. 22, nom. illeg. (Art. 52.1).

$\equiv$ Sium apium Roth, 1788, Tent. Fl. Germ. 1: 128, nom. illeg. (Art. 52.1).

三Apium maritimum Salisb., 1796, Prodr. Stirp. Chap. Allerton: 169, nom. illeg. (Art. 52.1).

$\equiv$ Sium graveolens (L.) Vest, 1806, Man. Bot.: 517.

$\equiv$ Selinum graveolens (L.) E. H. L. Krause, 1904, in Sturm (ed.), Deutschl. Fl. (ed. 2) 12: 38, fig. 4, nom. illeg. (Art. 53.1), non Vest (1806).

$\equiv$ Carum graveolens (L.) Koso-Pol., 1916, Bull. Soc. Imp. Naturalistes Moscou, s.n. 29: 199.

Type: EUROPE. "In Europae humectis, praesertim maritimis, 374.3" (lectotype LINN, designated by Tardieu-Blot, 1967: 44); "Herb. Clifford, 107, Apium 1" (epitype BM-CLIFF, designated by Reduron, Jarvis, 1992: 557; see also: Reduron, 2007).

= Helosciadium ruta DC., 1830, Prodr. 4: 106.

Type: S. AFRICA. "Ad Cap. Bonae-Spei" (holotype G).

= Apium integrilobum Hayata, 1911, J. Coll. Sci. Univ. Tokyo 30, 1 (Mater. Fl. Formosa): 126.

Type: CHINA. "[Taiwan] Taihoku, Maruyama [In humidis Maruyama], 05. 1903, Faurie 122" (holotype TAIF).

Distribution in China: NW: Xinjiang Weiwuer A. R., Gansu; N: Ningxia Huizu A.R., Shaanxi, Shanxi, Hebei, Shandong; NE: Liaoning; Centr.: Henan, Jiangsu, Anhui, Hubei, Hunan, Jiangxi; SW: Sichuan, Yunnan; S: Taiwan, Guangxi Zhuang A.R., Guangdong.

Distribution outside China: Russia, Japan, Indonesia, Malaysia, Vietnam, Myanmar, Bhutan, 
Nepal, India, Sri-Lanka, Pakistan, Kazakhstan, Kirghizia, Tajikistan, Uzbekistan, Turkmenistan, Afghanistan, Iran, Iraq, Oman, Saudi Arabia, Yemen, UAE, Bahrain, Lebanon, Syria, Israel, Jordan, Egypt, Cyprus, Greece (E Aegean Ils). Europe, Africa, N. America, S. America, Australia and Oceania.

Arcuatopterus filipedicellus Sheh Menglan et Shan Renhwa, 1986, Bull. Bot. Res., Harbin 6(4): 12, fig. 1.

Type: CHINA. "Yunnan: Binchuan Jizushan Zhushensi, alt. 2600 m, 09. 09. 1984, Pan Zehui 84-36" (holotype NAS!; isotype KUN!).

Distribution in China: Tibet: Xizang A. R.; SW: Yunnan. Endemic.

Arcuatopterus linearifolius Sheh Menglan et Shan Renhwa, 1986, Bull. Bot. Res., Harbin 6(4): 14, fig. 2.

Type: CHINA. "Yunnan: Binchuan Jizushan Zhushensi, alt. $2700 \mathrm{~m}, 09$. 09. 1984, Pan Zehui 84-35" (holotype NAS!; isotype KUN!).

Distribution in China: SW: Sichuan, Yunnan. Endemic.

Arcuatopterus thalictrioideus Sheh Menglan et Shan Renhwa, 1986, Bull. Bot. Res., Harbin 6, 4: 15, fig. 3, (thalictrioides).

Type: CHINA. "Sichuan: Muli, alt. 19002750 m, 20. 10. 1982, Pu Fating et Yao Gan 315" (holotype NAS!).

Distribution in China: Tibet: Xizang A. R.; SW: Sichuan, Yunnan. Endemic.

Aulacospermum anomalum (Ledeb.) Ledeb., 1833, Fl. Altaic. 4: 335.

$\equiv$ Cnidium anomalum Ledeb., 1829, Fl. Altaic. 1: 330 .

$\equiv$ Pleurospermum anomalum (Ledeb.) B. Fedtsch., 1915, Rastit. Turkest.: 604.

Type: RUSSIA. "[Altai] In pratis fertilibus humidiusculis in convallibus fluviorum Tscharysch, Koksun, Ledebour” (syntypes H!, LE!, P!); “[Altai] Prope redout Alexandrovsk, 20. 07. 1826, Meyer 243" (lectotype LE!, designated by Vinogradova, 2005: 961; isolectotype $\mathrm{P}$ !).

= Cnidium anomalum Ledeb. var. acaulis Ledeb., 1829, Fl. Altaic. 1: 330.

Type: RUSSIA. "In alpe ad fontes fl. Tschegan [In summa alpe Tscheganensi], 1826, Bunge" (holotype LE!)

= Aulacospermum schischkinii V. M. Vinogr., 2002,Bot. Zhurn. (St. Perersburg) 87(2): 141, fig. 1, 2A, map 3,2.
Type: KAZAKHSTAN. "Kazachstania, area monticulosa Centrali-Kazachstanica, prov. Karaganda, $70 \mathrm{~km}$ ad austro-orientem a urb. Karkaralinsk, mons Koschubai, 1559 m s.m. (systema montium Kent), 19. 07. 1968, Karamyscheva, Vlasov, Raczkowskaja 814" (holotype LE!).

- Aulacospermum rupestre auct. non Popov: Korovin, 1963, in Pavlov, Fl. Kazakhst. 6: 365, p.p.

Distribution in China: NW: Xinjiang Weiwuer A. R.

Distribution outside China: Russia, Mongolia, Kazakhstan.

Aulacospermum gonocaulum Popov, 1935, Byull. Moskovsk. Obšč. Isp. Prir. n.s. 44(2): 129.

Type: KAZAKHSTAN. "Jugum Transili Alatau, declivum dextrum rupestre rivulum Alma-Atinka minoris, infra Medeo, inter arbores et frutices, 18. 06. 1933, Popov" (lectotype MW!; designated here; isolectotype AA!).

- Aulacospermum anomalum auct. non Ledeb.: Schischk., 1950, in Schischkin (ed.), Fl. URSS 16: 241, p.p.

Distribution in China: NW: Xinjiang Weiwuer A. R.

Distribution outside China: Kazakhstan, Kirghizia.

Aulacospermum simplex Rupr., 1869, Mém. Acad. Imp. Sci. Saint-Petersbourg (sér. 7) 14 (4) (Sert. Tiansch.): 49.

三Pleurospermum simplex (Rupr.) Drude, 1898, in Engler \& Prantl, Nat. Pflanzenfam. 3(8): 172 (cum auct. Benth. et Hook. f.).

Type: KIRGHIZIA. "Tian-Schan, TaschrobatSchlucht, 04. 08. 1867, Osten-Saken" (lectotype LE!, designated by Kljuykov et al., 1976b: 63).

= Albertia commutata Regel et Schmalh., 1878, Trudy Imp. S.-Peterburgsk. Bot. Sada 5(2): 604.

= Trachydium commutatum (Regel et Schmalh.) M. Hiroe, 1979, Umbelliferae World: 713.

Type: KAZAKHSTAN. "In montibus alatavicis cisiliensibus, Karelin 392" (syntype LE!); "In montium alatavicorum jugo Kurmety et Keskelen, Semenov" (syntype LE); "In jugo Ketmen montium Thian-Schan, Kuschakevicz" (lectotype LE!, designated by Vinogradova, 1999: 81). KIRGHIZIA."In Kokaniae montium jugo Karakasuk et Kawuk, O. A. Fedtschenko" (syntype LE).

= Aulacospermum rupestre Popov, 1935, Byull. Moskovsk. Obšč. Isp. Prir. n.s. 44(2): 130.

= Pleurospermum rupestre (Popov) Fu Kuntsun et Ho Yehchi, 1979, in Fl. Reipubl. Popularis Sin. 55(1): 163. 
Type: KAZAKHSTAN. "In ripa dextra fluminis Alma-Atinka minor infra Medeo. 23. 06. 1933, Popov" (lectotype LE!, designated by Vinogradova, 1997a: 96; isolectotype AA!).

Distribution in China: NW: Xinjiang Weiwuer A. R.

Distribution outside China: Kazakhstan, Kirghizia, Tajikistan.

Aulacospermum stylosum (C. B. Clarke) Rech. f. et Riedl, 1963, K. Danske Vid. Selsk., Biol. Skrift 13(4) (Symb. Afghan. 5): 28.

$\equiv$ Pleurospermum stylosum C. B. Clarke, 1879, in Hooker f. (ed.), Fl. Brit. India 2: 704.

Type: INDIA. "Lahul, 11-12000 ft., Jaeschke" (syntype K!); "Kashmir, Falconer 3150" (lectotype $\mathrm{K}$ !, barcode K000685371, designated here).

= Hymenolaena darwasica Lipsky, 1900, Trudy Imp. S.-Peterburgsk. Bot. Sada 18(1): 72.

$=$ Pleurospermum darwasicum (Lipsky) B. Fedtsch., 1915, Rastit. Turkest.: 604.

$=$ Aulacospermum darwasicum (Lipsky) Schischk., 1950, in Schischkin (ed.), Fl. URSS 16: 243, tab. 15, fig. 14.

Type: TAJIKISTAN. "Buchara, Darwas, jugum Masar, ad marginem glac. Abdul-Gasan, 23. 07. 1899, Lipsky 184" (syntype LE!); "Buchara, Darwas, Gulbeda, 11400', 15. 07. 1899, Lipsky 183" (lectotype LE!, designated by Vinogradova, 1997b: 54). A. R.

Distribution in China: NW: Xinjiang Weiwuer

Distribution outside China: India, Pakistan, Tajikistan, Afghanistan.

Berula erecta (Huds.) Coville, 1893, Contr. U.S. Natl. Herb. 4: 115. 103.

इSium erectum Huds., 1762 (I-VI),Fl. Angl.:

三Sium berula Gouan, 1762, F1. Monsp.: 218.

$\equiv$ Sium incisum Pers., 1805, Syn. Pl. 1: 316., nom. illeg. (Art 52.1).

$\equiv$ Apium berula (Gouan) Caruel, 1889, in Parlatore, Fl. Ital. 8: 463.

$\equiv$ Selinum berula (Gouan) E. H. L. Krause, 1904, in Sturm (ed.), Deutschl. Fl. (ed. 2) 12: 32.

$\equiv$ Siella erecta (Huds.) Pimenov, 1978, Bot. Zhurn. (Leningrad) 63(12): 1746.

Type: Sium majus angustifolium. The lesser water Parsnep: p. 256 in Gerard, 1636, The Herball or Generall historie of plants (lectotype, designated by Reduron, 2007: 488).

三Sium angustifolium L., 1762 (VII-IX), Sp. Pl. (ed. 2) 2: 1672, nom. illeg. (Art. 52.1). $\equiv$ Berula angustifolia (L.) Mert. et W. D. J. Koch, 1826, in Mertens \& W. D. J. Koch (eds.), Röhl. Deutschl. Fl. (ed. 3) 2: 433, nom. illeg. (Art. 52.1).

$\equiv$ Berla monspelensium Bubani, 1900, Fl. Pyren. 2: 357, nom. illeg. (Art. 52.1).

Type: EUROPE. "In Europae australis aquosis nec extra aquis adscendit [In Pyr. Catal. ad Gerona] 09. 07. 1851. Bubani (?) 355.4".

= Sium erectum Huds. var. stenodon Koso-Pol., 1914, Bull. Soc. Imp. Naturalistes Moscou 28: 177.

$=$ Berula orientalis Woronow ex Schischk., 1950, in Schischkin (ed.), Fl. URSS 16: 467.

= Sium orientale (Woronow ex Schischk.) Soó, 1965, Acta Bot. Acad. Sci. Hung. 11, 1-2: 244.

Type: IRAN. "In Shagnis, in scabuigines cires Khoi, prov. Aderbeidzan, 11. 06. 1828, Szovits 437" (holotype LE!).

Distribution in China: NW: Xinjiang Weiwuer A. R., Gansu.

Distribution outside China: Russia, India, Pakistan, Kazakhstan, Kirghizia, Tajikistan, Uzbekistan, Turkmenistan, Afghanistan, Iran, Azerbaijan, Georgia, Armenia, Turkey, Iraq, Saudi Arabia, Yemen, Syria, Israel, Jordan. Europe, Africa, N. America, Australia and Oceania.

Bupleurum aitchisonii (Boiss.) H. Wolff, 1910, in Engler (ed.), Pflanzenreich 43(IV, 228): 142.

$\equiv$ Bupleurum olympicum Boiss. var. aitchisonii Boiss., 1888, Fl. Orient. Suppl.: 252.

Type: AFGHANISTAN. "Kurram valley, Afghanistan. Amongst stones at 12000 to 14000 feet, Sikaram, 08. 1878, Aitchison 929" (lectotype G-Bois.!, designated here by Pimenov, Jacquemoud; isolectotypes BM!, barcode BM000885377, C!, barcode C10008345, DD!, GH!, barcode GH00075432, K!, LE!, P!, barcode P03378462).

$=$ Bupleurum aitchisonii (Boiss.) H. Wolff var. abbreviatum H. Wolff, 1910, in Engler (ed.), Pflanzenreich 43(IV, 228): 143.

Type: AFGHANISTAN. "Afghanistan: auf Alpen des Kuram-Thales, Aitchison 929".

Distribution in China: Tibet: Xizang A. R.

Distribution outside China: India, Pakistan, Tajikistan, Afghanistan.

Bupleurum alatum Shan Renhwa et Sheh Menglan, 1979, in Fl. Reipubl. Popularis Sin. 55(1): 299, 256, tab. 138, fig. 1-6.

Type: CHINA. "Tibet, Nelamu Hsien, alt. 3900 m, 04. 09. 1972, Tibetan medicinal plant collection 1770" (holotype PE).

Distribution in China: Tibet: Xizang A. R. Endemic. 
Bupleurum amplexicaule (Wu Zhengyi ex Shan Renhwa et Li Yin) He Xingjin \& Wang Changbao, 2011, J. Syst. Evol. 49(6): 585, fig. 6, B, b.

$\equiv$ Bupleurum longicaule Wall. ex DC. var. amplexicaule Wu Zhengyi ex Shan Renhwa et Li Yin, 1974, Acta Phytotax. Sin. 12(3): 277.

Type: CHINA. "Yunnan, A-tun-tze [Deqin], 2700 m, 1935-09, Wang Qiwu 70007" (holotype PE!; isotype NAS).

Distribution in China: Tibet: Xizang A. R.; SW: Sichuan, Yunnan. Endemic.

Bupleurum baimaense Ma Xiangguang et $\mathrm{He}$ Xingjin, 2013, Ann. Bot. Fennici 50(6): 379.

Type: CHINA. "Yunnan province, Deqin County, Bai-ma Mountain, screes, alt. 4400 m, 19. 10. 2012, Ma Xiangguang 12101901" (holotype SZ).

Distribution in China: SW: Yunnan. Endemic.

Bupleurum bicaule Helm, 1809.Mém. Soc. Imp. Naturalistes Moscou 2: 108, tab. 8.

三Bupleurum falcatum L. var. bicaule (Helm) $\mathrm{H}$. Wolff, 1910, in Engler (ed.), Pflanzenreich 43(IV, 228): 140 .

三 Bupleurum falcatum L. subsp. bicaule (Helm) Koso-Pol., 1915, Trudy Imp. S.-Peterburgsk. Bot. Sada 30, 2 (Epitome Bupleur. Ross.): 217.

Type: RUSSIA. "In rupibus montibus prope Krasnojarsk, Helm" (holotype MW - destr.).

= Bupleurum baldense Turra var. multicaule Ledeb., 1829, Fl. Altaic. 1: 350.

= Bupleurum exaltatum M. Bieb. var. multicaule (Ledeb.) Ledeb., 1844, F1. Ross. 2: 266, p.p.

Type: RUSSIA. "[Altai] In locis subsalsis deserti editi ad fl. Tschuja, 1826, Bunge 224b" (lectotype LE!, designated here; isolectotype P!).

= Bupleurum pusillum Krylov, 1903, Trudy Imp. S.-Peterburgsk. Bot. Sada 21(1): 18.

= Bupleurum bicaule Helm var. pusillum (Krylov) Gubanov, 1983, Fl. Vostoch. Khangaya: 143.

Type: RUSSIA. "In montibus Altaicis orientalibus, locis sterilimis deserti Tschujensis, ad fl. Tschegan-Usun, Dshölo etc., nec non in Mongolia boreali - in declivibus australibus jugi Tannu-Ola ad fl. Ak-Karassuk [Altai, inter flumina Chegan-Usun et Elangash, in collibus continuis erraticis,] 02. 07. 1898, Saposhnikov" (lectotype TK!, designated by Polozhij, Balaschova, 1989: 26; isolectotype LE!); "Altai, vallis fl. Chegan-Usun infra ostium fl. Dshölo. 06. 07. 1901, Krylov" (syntype LE!); "Altai, trajectum in fontes fluminis Dshölo. 29. 06. 1897, Saposhnikov" (syntype TK).
- Bupleurum baldense auct. non Turra: Ledeb., 1829, F1. Altaic. 1: 350.

Type: RUSSIA. "[Altai] In locis subsalsis deserti editi ad fl. Tschuja, 1826, Bunge" (lectotype LE!, designated here; isolectotype P!).

Distribution in China: Tibet: Xizang A. R., Qinghai; N: Neimenggu A. R., Ningxia Huizu A. R., Shaanxi, Shanxi, Hebei; NE: Heilongjiang, Jilin.

Distribution outside China: Russia, Mongolia, Kazakhstan.

Bupleurum bicaule Helm var. latifolium $\mathrm{Chu}$ Youchang, 1977, P1. Herb. Chin. Bor.-Or. 6: 293, 195.

Type: CHINA. "Heilongjiang, Huma, 06. 08. 1950, Han 700" (holotype IFP).

Distribution in China: NE: Heilongjiang. Endemic.

Bupleurum boissieuanum H. Wolff, 1929, Repert. Spec. Nov. Regni Veg. 27: 186.

Type: CHINA. "Su-tchuen orientalis: district de Tchen Kéou-Tin, Farges" (lectotype P!, barcode P00752924, designated here; isolectotypes P!, barcodes P00752922, P00752923).

= Bupleurum longeradiatum Turcz. var. porphyranthum Shan Renhwa et Li Yin, 1974, Acta Phytotax. Sin. 12, 3: 270.

Type: CHINA. "Sichuan, Chengkou, Dai TienLan 1040831" (holotype NAS).

Distribution in China: NW: Gansu; Tibet: Qinghai; N: Ningxia Huizu A.R., Shaanxi; Centr.: Henan, Hubei; SW: Sichuan. Endemic.

Bupleurum candollei Wall. ex DC., 1830, Prodr. 4: 131, "Candollii".

Type: NEPAL. "In Nepalia ad Gossaingsthan et Emodum, 1829, Wallich [552]" (lectotype G-DC!, barcode G00367696, designated here; isolectotypes: BM!, barcode BM000622299, CAL, E!, barcodes E00000338, E00000339, K-WALLICH!, barcodes K000687102, K001111250, K001111251).

=Bupleurum candollei Wall. ex DC. f. acutifolium H. Wolff, 1910, in Engler (ed.), Pflanzenreich 43(IV, 228): 150 .

Type: INDIA. "Dhurmsala, 10000", 17. 10. 1874, Clarke 24013" (syntypes K!, LE!); "NW India, Duthie 1150, 1152" (syntypes K, LE!).

= Bupleurum candollei Wall. ex DC. f. obtusifolium H. Wolff, 1910, in Engler (ed.), Pflanzenreich 43(IV, 228): 150.

Type: INDIA. "Wallich 552" (syntypes K!, LE!); "Hügel Asien, Strachey, Winterbottom 405" 
(syntype); "Sikkim, Phullalong, 26. 10. 1875, Clarke 25689" (syntypes K!, LE!); "Tihri Garhwal, Ganges valley above Jangla, 10-11000', 16. 07. 1883, Duthie 1150" (syntypes CAL, LE!).

Distribution in China: Tibet: Xizang A. R.; SW: Sichuan, Yunnan.

Distribution outside China: Thailand, Myanmar, Bhutan, Nepal, India, Pakistan.

Bupleurum candollei Wall. ex DC. var. atropurpureum $\mathrm{Wu}$ Zhengyi ex Shan Renhwa et Li Yin, 1974, Acta Phytotax. Sin. 12(3): 275.

$\equiv$ Bupleurum atropurpureum (Wu Zhengyi ex Shan Renhwa et Li Yin) Wu Zhengyi, 1984, Index Fl. Yunnan. 1: 906.

Type: CHINA. "Yunnan, Zhangkang Xian (= Yangde Xian), Daxue Shan, alt. 2000 m, grassy slope, 23. 07. 1937, T. T. Yu 16918" (holotype PE; isotypes $\mathrm{E}, \mathrm{KUN})$.

Distribution in China: SW: Yunnan. Endemic.

Bupleurum candollei Wall. ex DC. var. paucefulcrans (Wu Zhengyi ex Shan Renhwa et Li Yin) He Xingjin et Wang ChangBao, 2011, Nordic J. Bot. 29: 429, fig. 5.

$\equiv$ Bupleurum tenue Buch.-Ham. ex D. Don. var. paucefulcrans $\mathrm{Wu}$ Zhengyi ex Shan Renhwa et Li Yin, 1974, Acta Phytotax. Sin. 12(3): 291.

$\equiv$ Bupleurum hamiltonii N. P. Balakr. var. paucefulcrans (Wu Zhengyi ex Shan Renhwa et Li Yin) Sheh Menglan et M. F. Watson, 2005, in Flora of China 14: 72.

Type: CHINA. "Guizhou, Bijei, 1300 m, Yu PingHu 560" (holotype KUN).

Distribution in China: SW: Guizhou. Endemic.

Bupleurum candollei Wall. ex DC. var. virgatissimum $\mathrm{Wu}$ Zhengyi ex Shan Renhwa et $\mathrm{Li}$ Yin, 1974, Acta Phytotax. Sin. 12(3): 275.

Type: CHINA. "Sichuan, Hui Dong, 23. 06. 1937, Wu SuGong, 1833" (holotype KUN).

Distribution in China: SW: Sichuan, Yunnan. Endemic.

Bupleurum chaishoui Shan Renhwa et Sheh Menglan, 1979, in Fl. Reipubl. Popularis Sin. 55(1): 299, 286, tab. 155, fig. 1-8.

Type: CHINA. "Szechuan, Mao-Wan Hsien, alt. 2700 m, 08. 10. 1976, T. T. Chang, Y. C. Lan”.

Distribution in China: SW: Sichuan. Endemic.

Bupleurum chinense DC., 1830, Prodr. 4: $128 .$.

Type: CHINA. "In China. Stannton" (holotype G-DC!).
= Bupleurum chinense Franch., 1883, Nuov. Arch. Mus. Hist. Nat. (sér. 2) 6 (Pl. David. 1): 138.

Type: CHINA. "Environs de Pekin, 07.1863, David 454" (holotype P!, barcode P00752825).

= Bupleurum pekinense Franch. ex Forbes et Hemsl., 1887, J. Linn. Soc. Bot. 23: 327, nomen.

= Bupleurum chinense DC. f. pekinense (Franch. ex Forbes et Hemsl.) Shan Renhwa et Li Yin, 1974, Acta Phytotax. Sin. 12(3): 293.

= Bupleurum scorzonerifolium Willd. f. latum (H. Wolff) Nakai, 1937, J. Jap. Bot. 13(7): 486.

Type: KOREA. "Quelpaert: in herbidis lateris montis Hallusan, 30. 10. 1917, Nakai 6063" (syntype TI); "Prov. Keiki: in monte Nankanzan, 18. 10. 1900, Uchiyama" (syntype TI); "Prov. Keiki: in monte Kakuozan, Suigen, 08. 09. 1912, Uyeki 530" (syntype TI); "Prov. Keiki. Syoto, 23. 10. 1900, Uchiyama" (syntype TI); "Prov. Kogen: Bokuto, 02. 08. 1902, Uchiyama" (syntype TI); "Prov. Heinan: Chinnampo, 21. 09. 1915, Nakai 2963" (syntype TI); "Prov. Kennan: inter Toikori et Sanyo, 21. 07. 1914, T. Nakai, 3597" (syntype TI); "Korea Prov. Kennan: in monte circa Tyosin. 09. 07. 1914, Nakai 9202" (syntype TI).

= Bupleurum togasii Kitag., 1951, J. Jap. Bot. 26(6): 15.

Type: CHINA. "China bor., prov. Shan-shi: in monte Wu-tai-shan, 25. 07. 1942, Togasi 912" (holotype TNS!).

Distribution in China: NW: Gansu; Tibet: Qinghai; N: Neimenggu A. R., Ningxia Huizu A.R., Shaanxi, Shanxi, Hebei, Shandong; NE: Heilongjiang, Jilin, Liaoning; Centr.: Henan, Jiangsu, Anhui, Hubei, Hunan, Jiangxi, Zhejiang, Fujiang; SW: Sichuan.

Distribution outside China: Japan, Korea.

Bupleurum chinense DC. f. octoradiatum (Bunge) Shan Renhwa et Shen Menglan ex Li Yin et Sheh Menglan, 1979, in Fl. Reipubl. Popularis Sin. 55(1): 293.

三 Bupleurum octoradiatum Bunge, 1833, Mém. Acad. Imp. Sci. St.-Petersburg Divers Savans 2: 106.

$\equiv$ Bupleurum chinense DC. var. octoradiatum (Bunge) Kitag., 1960, Bull. Natl. Sci. Mus. 5, 1 (46): 10.

Type: CHINA. "In montosis Zui-weg-schan, 1831, Bunge" (holotype P!).

Distribution in China: N: Shanxi, Hebei. Endemic.

Bupleurum commelynoideum H. Boissieu, 1902, Bull. Herb. Boissier (2 sér.) 2: 805. 
Type: CHINA. "Thibet oriental, princ. de Kiala, Ta-Tsien-Lou, 10.08.1894. Soulié 2600" (lectotype P!, barcode P00752821, designated here; isolectotype E!); ibid., "1893. Soulié 124, 639, 702" (syntypes P!, barcodes P00752816, P00752818, P00753046); "West Szechuan and Tibetan frontier: chiefly near Tachienlu, at 9000-13500 feed, 12,1890. Pratt 618" (syntype P!, barcode P00752822); "Chine occidentale, Se-Tschuen occidental: Tatsienlou, Mussot" (syntype P!, barcode P00752824).

= Bupleurum commelynoideum H. Boissieu var. elatissimum H. Boissieu, 1906, Bull. Soc. Bot. France 53: 424.

Type: CHINA. "Thibet oriental, princ. de Kiala, Ta-Tsien-Lou, Soulié 2596" (holotype P!).

Distribution in China: NW: Gansu; Tibet: Xizang A. R., Qinghai; N: Ningxia Huizu A. R.; SW: Sichuan, Yunnan. Endemic.

Bupleurum condensatum Shan Renhwa et $\mathrm{Li}$ Yin, 1974, Acta Phytotax. Sin. 12(3): 279, tab. 56, fig. 1-9.

Type: CHINA. "Qinghai: Gonghe Xian, Deheba, Yao Zhongwu 875" (holotype NAS).

Distribution in China: Tibet: Qinghai. Endemic.

Bupleurum densiflorum Rupr., 1869, Mém. Acad. Imp. Sci. Saint-Petersbourg (sér. 7) 14(4) (Sert. Tiansch.): 47.

三Bupleurum falcatum L. var. densiflorum (Rupr.) Koso-Pol., 1915, Trudy Imp. S.-Peterburgsk. Bot. Sada 30(2) (Epitome Bupleur. Ross.): 226.

Type: KIRGHIZIA. "In regione alpina jugi Thian-Schan, am Tschatyrkol, 26. 07. 1867, OstenSaken" (lectotype LE!, designated by Linczevski, 1950: 304).

= Bupleurum kokanicum Regel et Schmalh., 1881, Izv. Obšč. Ljubit. Estestv. 34, 2 (Descr. P1. Nov. Rar. Fedtsch.): 29.

Type: KIRGHIZIA. "In Kokaniae trajectu Dschiptyk 9-12000“ alt., 22. 06. 1871, O. A. Fedtschenko" (lectotype LE!, designated by I. A. Linczevski (Vinogradova, 1997a: 98).

= Bupleurum mongolicum V. M. Vinogr., 1985, Novosti Sist. Vyssh. Rast. 22: 194.

Type: MONGOLIA. "Dzhungaria, $110 \mathrm{~km}$ ad meridiem et austro occidentem a distr. Bulgan, jugum Baitag-Bogdo, declive generele boreale, 2650 m s.m., steppa caricoso-petrophyto-varieherbosofestucosa, 08. 08. 1977, Volkova, Raczkovskaya 7283" (holotype LE!).
- Bupleurum triradiatum auct. non Adams: Kar. \& Kir., 1842, Bull. Soc. Imp. Naturalistes Moscou 15: 361 .

- Bupleurum ranunculoides auct. non L.: Regel \& Herder, 1866, Bull. Soc. Imp. Naturalistes Moscou 39(3): 74.

Distribution in China: NW: Xinjiang Weiwuer A. R.; Tibet: Xizang A. R., Qinghai.

Distribution outside China: Mongolia, Kazakhstan, Kirghizia, Tajikistan.

Bupleurum dielsianum $\mathrm{H}$. Wolff, 1910, in Engler (ed.), Pflanzenreich 43(IV, 228): 147, fig. 16, E.

Type: CHINA. "Im Norden der Provinz Shensi: T'ai-pa-shan [China inferior, provincia Schensi septentr., in alto monte Thai pai-san], Giraldi 5833" (lectotype FI!, designated here); "N Shensi: Gua-inshan, 20. 07. 1898, Giraldi 5754" (syntype FI!).

Distribution in China: N: Shaanxi. Endemic.

Bupleurum dracaenoides Wang Huanchong, $\mathrm{He}$ Zhaorong et Sun Hang, 2014, Syst. Bot. 38(4): 1192.

Type: CHINA. "China, Yunnan province: Luquan County, near Xueshan village, Puduhe River valley, 2300-2700 m, on steep limestone cliffs, 26. 09. 2007, Wang Huanchong et al. 1611" (holotype YUKU; isotypes E, K, KUN, MO, YUKU).

Distribution in China: SW: Sichuan, Yunnan. Endemic.

Bupleurum euphorbioides Nakai, 1914, Bot. Mag. (Tokyo) 28: 313.

Type: KOREA. "Prov. Ham-gyong: in monte Pailtusan, 10. 08. 1914, Nakai 9199" (lectotype TI!, designated by Pimenov, in Pimenov, Ostroumova, 2012: 53); "Prov. Kan-uon: in summo montis Kumgangsan, 08. 1914, Mori 41" (syntype).

$=$ Bupleurum tatudinense A.I.Baranov, 1954, Acta Soc. Harbin Investig. Nat. Ethnogr., Bot. 12: 32, fig. 31-34.

Type: CHINA. "Prov. Sungkiang, distr. Vei-cha, Mt. Tatoudintsu, 24. 08. 1953, Baranov".

= Bupleurum euphorbioides Nakai var. alpestre Sakata, 1958, J. Jap. Bot. 33: 30.

Type: KOREA. "Korea septentrionalis: in regione alpina Mt. Shajitsuho, 1936, Sakata".

Distribution in China: NE: Heilongjiang, Jilin, Liaoning.

Distribution outside China: Russia, Korea.

Bupleurum exaltatum M. Bieb., 1800, Beschr. Casp.: 156. 1798; Tab. Prov. Casp: 113. 
$\equiv$ Buprestis exaltata (M. Bieb.) Spreng., 1812, Mag. Neuersten Entdesk. Gesammten Naturk. Ges. Naturf. Freude Berlin 6: 259.

$\equiv$ Bupleurum falcatum L. subsp. exaltatum (M. Bieb.) Rouy et Camus ex Briq., 1897, Monogr. Bupleurum Alpes Marit.: 94, p.p.

$\equiv$ Bupleurum falcatum L. var. euexaltatum $\mathrm{H}$. Wolff, 1910, in Engler (ed.), Pflanzenreich 43(IV, 228): 134, nom. illeg. (Art. 52.1).

Type: "In pratis maritimis et ad latere collium, in Tauria, quam in promontoriis Caucasico minime rarum, von Marchall Bieberstein" (lectotype LE, designated here; isolectotype B-Willd!).

= Bupleurum angustifolium Pall., 1771, Reise Stotth. Russ. Reich 1: 345, nom. inval.

$=$ Bupleurum graminifolium Georgi, 1800, Beschr. Russ. Reich 3, 4: 828, nom. illeg., non Vahl. (1794), nec Salisb. (1796).

= Bupleurum sibthorpianum Sm., 1806, in Sibth. et Sm., Fl. Graec. Prodr. 1: 179.

Type: EUROPE, GREECE. "In Peloponesso, Sibthorp" (holotype OXF!).

= Bupleurum linearifolium DC., 1830, Prodr. 4: 131.

= Bupleurum exaltatum M. Bieb. var. linearifolium (DC.) Boiss., 1844. Ann. Sci. Nat. Paris (sér. 3, Bot.) 1 (Pl. Aucher.): 150.

$=$ Bupleurum falcatum L. var. linearifolium (DC.) Boiss. ex H. Wolff, 1910, in Engler (ed.), Pflanzenreich 43(IV, 228): 135.

Type: IRAN. "In argillosis montium circa Badalan, distr. Khoi, prov. Aderbeidzhan Persiae, 20. 07. 1828, Szovits 560" (lectotype G-DC!, designated here; isolectotypes JE, LE!).

= Bupleurum cernuum Ten., 1829, Fl. Napol. 3: 270, tab. 125.

= Bupleurum falcatum L. subsp. cernuum (Ten.) Arcangeli, 1894, Comp. Fl. Ital. (ed. 2): 590.

= Bupleurum falcatum L. var. cernuum $($ Ten.) $\mathrm{H}$. Wolff, 1910, in Engler (ed.), Pflanzenreich 43(IV, 228): 136.

Type: EUROPE, ITALY. 'E comune ne' monti di Abruzzo e del Sannio; alla Manella. al Morrone, a monte Corno, a Rocca Valle oscura, alla difesa di Pettorano, a Monte carpaco, a Monte di Mezza ec. [Tenore 184?]" (syntype FI!).

= Bupleurum schimperi Boiss., 1844, Ann. Sci. Nat. Paris (sér. 3, Bot.) 1 (Pl. Aucher.): 151.

$=$ Bupleurum linearifolium DC. var. schimperi (Boiss.) Boiss., 1872, Fl. Orient. 2: 850, "schimperianum".

Type: EGYPT (SINAI). "In Arabiâ petraeâ [In rupestribus regionis Raphidim Arabiae petr.], 25.
04. 1835, Schimper 290 [299]" (lectotype G-BOIS!, designated here by Pimenov, Jacquemoud; isolectotypes A, E!, barcode E00000327, FI!, JE!, barcode JE00003950, GH, K!, barcode K000687140, LE!, P!, S!, STU, TUB).

= Bupleurum kotschyanum Boiss., 1849, Diagn. P1. Orient.(ser. 1) 10: 29.

= Bupleurum linearifolium DC. var. kotschyanum (Boiss.) Boiss., 1872, Fl. Orient. 2: 850.

Type: IRAN. "Ad radices m. Demawend pr. p. Lar, 04. 08. 1843, Kotschy 627" (lectotype G-BOIS!, designated here by Pimenov and Jacquemoud; isolectotypes JE!, P!); "In m. Elburs ("Elbrus"), pr. pagum Passgala, 1843, Kotschy 649" (syntypes B-BOIS!, P!, WAG).

= Bupleurum cuspidatum Bunge, 1852, in Beitr. Fl. Russl. (Reliq. Bot.): 123 (299).

Type: UZBEKISTAN. "An Felsenwanden des oberen Sarafschan, 10. 09. 1841, Lehmann 527" (lectotype P!, designated here; isolectotype LE!).

= Bupleurum exaltatum M. Bieb. var. microlepis Fenzl ex Tchih., 1860, As. min., Bot. 1: 424.

Type: LEBANON. "Syria libanotica: fruticetis supra Edem versus Dania alt. 1786 m" (syntype). EGYPT. "Arabia petraea: pr. Raphidem, Schimper 290" (syntype).

= Bupleurum exaltatum M. Bieb. var. macropodum Fenzl ex Tchih., 1860, As. min., Bot. 1: 424 .

Type: IRAN. "Persia: ad radices montium Elburs ["Elbrus"] et Damavend, atque in rupestribus prope cataractas Ser-Abi-Schir, Kotschy".

$=$ Bupleurum exaltatum M. Bieb. var. quinquinerve Fenzl ex Tchih., 1860, As. min., Bot. 1: 423.

Type: TURKEY. "Tauro Cilicico septentrionali, alt. 2275 m, Kotschy" (syntype); "Persia boreali, Szowits" (syntype); "Buhse" (syntype); "Aucher 4596 et 4598" (syntypes); "Persia austr., Kotschy 575 et 786 " (syntypes). IRAQ. "Assyria, Aucher 3636" (syntype). LEBANON. "Syria libanotica, Aucher 3621" (syntype). TURKMENISTAN. "Turkomania, Lehm[ann]" (syntype). UKRAINE. "Tauria". RUSSIA. "Caucasus, Hohen[acker]1836 et 2" (syntypes). KAZAKHSTAN. "Songaria, Karelin et Kirilow 1511" (syntype).

$=$ Bupleurum irregulare Boiss. et Kotschy, 1872, Fl. Orient. 2: 850.

Type: LEBANON. "In regione alpine Libani supra Edem et Dimam, alt 5500', Aucher-Eloy 3621" (syntypes BM!, G-BOIS!, P!); "Haut Liban, à Ain-Dahub, au pied de Mar-Sémone, audessus de Diman, 09. 06. 1864, Blanche 3222" 
(syntype JE); "Fruticosa supra Eden versus Danie occupat alt. 5500 ped., 28. 07. 1855, Kotschy 331" (lectotype G-BOIS!, designated here by Pimenov and Jacquemoud; isolectotypes BM!, GH, K!, MPU, US). SYRIA. "In subalpinis superioribus prope Hadet, 07. 1824, Ehrenberg 262" (syntype G-BOIS!).

- Bupleurum baldense auct. non Turra: Boiss., 1872, Fl. Orient. 2: 848.; Schmalhausen 1895: 390.

Distribution in China: NW: Xinjiang Weiwuer A. R.

Distribution outside China: Russia, Pakistan, Kazakhstan, Kirghizia, Tajikistan, Uzbekistan, Turkmenistan, Afghanistan, Iran, Azerbaijan, Georgia, Armenia, Turkey, Iraq, Saudi Arabia, Lebanon, Syria, Israel, Egypt (Sinai). Mediterranean Europe, Africa.

Bupleurum franchetii (H. Boissieu) He Xingjin et Wang Changbao, 2011, J. Syst. Evol. 49(6): 585, fig. $6, \mathrm{C}$, c.

$\equiv$ Bupleurum longicaule Wall. ex DC. var. franchetii $\mathrm{H}$. Boissieu, 1906, Bull. Soc. Bot. France 53: 425.

Type: CHINA. "Province de Yun-nan in pratis siccis montis Pee-ngay-tze supra Houang-kia-pin, 04. 09. 1882, Delavay 454" (syntype P!, barcode P03378388); "Yun-nan in pratis montis Heechan-men (Lang kong), alt. 3000 m, 01. 09. 1884, Delavay 937" (syntype P!, barcode P03378427); "Province de Yun-nan, les praeries de Songpin, audessus de Ta-pin-tze, $1800 \mathrm{~m}, 18.08$. 1885. Delavay 937" (syntype P!, barcodes P03378393, P03378387, P03378413); "Yun-nan in silvis ad Che-tcho-tze. Delavay 4582" (syntype P); "Su-tchuen oriental, a Tchen-kéeou- tin, Farges [1195] " (syntypes B!, BM!, barcode BM000703393, P!, barcode P03378414).

- Bupleurum candollei auct. non Wall. ex DC.: Franch., 1894, Bull. Annuel Soc. Philom. Paris (sér. 8) 6: 115 .

Distribution in China: NW: Gansu; Tibet: Qinghai; N: Shaanxi, Shanxi; Centr.: Henan, Hubei; SW: Sichuan, Yunnan. Endemic.

Bupleurum gansuense Pan Shengli et Hsu Pingsheng, 1995, Rheedea 5(2): 145.

Type: CHINA. "Yuzhong Country, Gansu Prov., NW China".

Distribution in China: NW: Gansu. Endemic.

Bupleurum giraldii (H. Wolff) Koso-Pol., 1915, Trudy Imp. S.-Peterburgsk. Bot. Sada 30(2) (Epitome Bupleur. Ross.): 164.
EBupleurum longicaule Wall. ex DC. var. giraldii H. Wolff, 1910, in Engler (ed.), Pflanzenreich 43(IV, 228): 123 , fig. 15 , A.

Type: CHINA. "Nordchina: "Nord-Shensi" [Qinling] Giraldi 1341" (syntype); "Shensi, Herb. Biondi 1321" (syntype FI?); "Shensi, Schlagintweit 6408" (syntype).

Distribution in China: Tibet: Qinghai; N: Shaanxi, Shanxi. Endemic.

Bupleurum gracilipes Diels, 1900, Bot. Jahrb. Syst. 29 (3-4) (Fl. Centr. China): 493.

Type: CHINA. "Setchuen, Nan ch'uan: Hei wan ai, Bergange, 25. 09. 1891, von Rosthorn" (holotype $\mathrm{O}$ !).

Distribution in China: SW: Sichuan. Endemic.

Note: In some recent Chinese publications this and next species, having similar scientific names are confused.

Bupleurum gracillimum Klotzsch, 1862, in Klotzsch \& Garcke (eds.), Bot. Ergebn. Reise Waldemar: 148.

$\equiv$ Bupleurum falcatum L. var. gracillimum (Klotzsch) H. Wolff, 1910, in Engler (ed.), Pflanzenreich 43(IV, 228): 132.

Type: INDIA. "Im Himalaya, Hoffmeister".

Distribution in China: Tibet: Xizang A. R.

Distribution outside China: Bhutan, Nepal, India, Pakistan, Afghanistan. Endemic to Central and Eastern Asia.

Bupleurum gulczense O. et B. Fedtsch., 1908, Trudy Imp. S.-Peterburgsk. Bot. Sada 28: 18.

Type: KIRGHIZIA. "Montes Alaici, inter Gulcza et Ljangar, 15. 08. 1901, O. A. Fedtschenko, B. A. Fedtschenko" (lectotype LE!, designated by Linczevski, 1950: 300).

Distribution in China: NW: Xinjiang Weiwuer A. R.

Distribution outside China: Kirghizia, Tajikistan.

Bupleurum hamiltonii N. P. Balakr., 1966, J. Bombay Nat. Hist. Soc. 63: 328.

$\equiv$ Bupleurum tenue Buch.-Ham. ex D. Don, Prodr. Fl. Nepal.: 182. 1825, nom. illeg., non Salisb. (1796).

Type: NEPAL. "Nepalia, Narainhetty, 08. 09. 1802, Buchanan-Hamilton" (lectotype BM!, designated here; isolectotypes CAL, E?).

= Bupleurum chevalieri Cherm., 1921, Bull. Soc. Bot. France 68: 510, fig. 135. 
Type: VIETNAM. "Annam: massif de Langbian-dran, 1000 m, 09. 12. 1914, Chevalier 30635" (holotype P!).

= Bupleurum tenue Buch.-Ham. ex D. Don var. attenuatum Nasir, 1954, Univ. Calif. Publ. Bot. 27(7): 437, fig. 9, c-d.

Type: PAKISTAN. "Bhadwar, Kangra, Punjab, alt. 2000 ft., 14. 05. 1933, Koelz 4453" (holotype US; isotype BM!).

Distribution in China: Tibet: Xizang A. R.; Centr.: Hubei; SW: Sichuan, Guizhou, Yunnan; S: Guangxi Zhuang A. R.

Distribution outside China: Malaysia, Vietnam, Thailand, Myanmar, Bhutan, Nepal, India, Pakistan.

Bupleurum hamiltonii N. P. Balakr. var. humile (Franch.) Shan Renhwa et Sheh Menglan, 1993, in Wang Wentsai (ed.), Vasc. Pl. Hengduan Mts.: 1306.

三Bupleurum tenue Buch.-Ham. ex D. Don var. humile Franch., 1894, Bull. Annuel Soc. Philom. Paris (sér. 8) 6: 118.

Type: CHINA. "Yunnan, in pascuis prope collum Hee-chan-man, 28. 10. 1889, Delavay 4233" (holotype $\mathrm{P}$ !; isotype $\mathrm{K}$ !).

Distribution in China: SW: Sichuan, Yunnan. Endemic.

Bupleurum kaoi Liu Tangshui, Chao Chuangying et Chuang Tsaniang, 1961, Quart. J. Taiwan Mus. 14(1-2): 22, tab. 3, 13.

Type: CHINA. "[Taiwan] Fuhsinli, Houlung, Miaoli Hsien, M. T. Kao K3746” (holotype TAI).

Distribution in China: S: Taiwan. Endemic.

Bupleurum komarovianum Lincz., 1950, in Schischkin (ed.), Fl. URSS 16: 319.

$\equiv$ Bupleurum chinense DC. var. komarovianum (Lincz.) Liou et Huang Yhuei, 1977, Fl. Pl. Herb. Chin. Bor.-Or. 6: 200.

$\equiv$ Bupleurum falcatum L. subsp. komarovianum (Lincz.) Vorosch., 1985, in Skvorzov (ed.), Florist. Issl. v raz. raionakh SSSR: 183.

Type: CHINA. "Mandshuria, in Quercetis in valle fl. Dzhurdecho, affuxinis fl. Mudandsian, prope Omoso, 30. 07. 1896, Komarov" (holotype LE!).

- Bupleurum falcatum auct. non L.: Lee, 1985, Illustr. Fl. Korea: 577, fig. 2308.

Distribution in China: NE: Heilongjiang, Jilin, Liaoning.

Distribution outside China: Russia, Korea.

Bupleurum krylovianum Schischk., 1935, in Krylov (ed.), Fl. Zapadnoi Sibiri 8: 2010. $\equiv$ Bupleurum falcatum L. var. oblongifolium Trautv., 1866, Bull. Soc. Imp. Naturalistes Moscou 39(2): 319.

$\equiv$ Bupleurum falcatum L. subsp. flexuosum Koso-Pol., 1915, Trudy Imp. S.-Peterburgsk. Bot. Sada 30(2) (Epitome Bupleur. Ross.): 221, p.p., non Bupleurum flexuosum Ledeb. s.str.

Type: KAZAKHSTAN. "In montibus Tarbagatai, 25. 08. 1841, Schrenk 457" (holotype LE!).

Distribution in China: NW: Xinjiang Weiwuer A. R.

Distribution outside China: Russia, Mongolia, Kazakhstan, Kirghizia.

Bupleurum kunmingense Li Yin et Pan Shengli, 1984, Acta Phytotax. Sin. 22(2): 131, fig. 1.

Type: CHINA. "Yunnan: Kunming, Yuanshan, alt. 2000 m, 01. 08. 1981, Pan Shengli 0232" (holotype SHPII; isotype KUN!).

Distribution in China: SW: Yunnan. Endemic.

Bupleurum kweichowense Shan Renhwa, 1940, Sinensia 11(1-2): 148, tab. 1.

Type: CHINA. "Kweichow, Fang Ching Shan, Hsing Shan, alt. $2100 \mathrm{~m}$, on exposed rocky ridges, 05. 10. 1931, Steward, C. Y. Chiao, H. C. Cheo 645" (lectotype K!, designated here; isolectotypes E!, LE!).

Distribution in China: SW: Guizhou. Endemic.

Bupleurum longeradiatum Turcz. [Bull. Soc. Imp. Naturalistes Moscou 11: 93. 1838, nom. inval. (Art. 39.1)], 1844, Bull. Soc. Imp. Naturalistes Moscou 17: 719.

$\equiv$ Bupleurum longeradiatum Turcz. var. genuinum H. Wolff, 1910, in Engler (ed.), Pflanzenreich 43(IV, 228): 55.

Type: RUSSIA. "In Dahuriae maxime orientali ad fluvia Schilka et Argun [In herbidis ad fl. Schilka], 1833, Turczaninow" (lectotype LE!, designated by Vinogradova, 2005: 960; isolectotypes B!, BP!, C!, H!, K!, KW-Turcz!, barcodes KW001000560, KW001000561, KW001000562, LE!, M!, barcode M0172805, MW!, NSK, barcode NSK0000239, P!, $\mathrm{TK})$

= Bupleurum longeradiatum Turcz. var. breviradiatum F. Schmidt ex Maxim., 1859, Mém. Acad. Imp. Sci. Saint-Petersbourg Divers Savants 9 (Prim. Fl. Amur.): 125.

= Bupleurum longeradiatum Turcz. f. breviradiatum (F. Schmidt ex Maxim.) Kitag., 1961, J. Jap. Bot. 36: 241.

Type: RUSSIA. "Küstenregion: Dorf Pronge an der Amur-Mündung, in hohen Grase. 05. 08. 1854, 
Maximowicz" (lectotype LE!, designated here); "Kuegra bei Nikolajevsk, gleicher Staford, 15. 08. 1854, Maximowicz" (syntype LE!).

= Bupleurum sachalinense F. Schmidt, 1868, Mém. Acad. Imp. Sci. Saint-Petersbourg (sér. 7) 12(2): 135.

= Bupleurum longeradiatum Turcz. var. sachalinense (F. Schmidt) H. Boissieu, 1910, Bull. Soc. Bot. France 57: 413, in nota.

= Bupleurum longeradiatum Turcz. subsp. sachalinense (F. Schmidt) Kitag., 1961, J. Jap. Bot. 36(8): 242.

Type: RUSSIA. "An trochenen Abhängen von Dui und Arkai an durch das ganze Gebiet haufig ... Auch um Hakodate (Dr. Albrecht) [Insula Sachalin, Arkai, 08. 09. 1860, Glehn" (lectotype LE!, designated by V. M. Vinogradova, 2006: 1282); "Dui, 18. 07. 1860, Glehn" (syntype LE!). JAPAN. "Japonia, insula Jesso, circa Hakodate, 1861, Albrecht" (syntype LE!)].

= Bupleurum leveillei $\mathrm{H}$. Boissieu, 1910, Bull. Soc. Bot. France 57: 413.

$\equiv$ Bupleurum longeradiatum Turcz. var. leveillei (H. Boissieu) Kitag., 1960, Bull. Natl. Sci. Mus. 5, 1 (46): 11.

$\equiv$ Bupleurum longeradiatum Turcz. f. leveillei (H. Boissieu) Kitag., 1961, J. Jap. Bot. 36(8): 241.

Type: KOREA. "Île de Quelpaert, in herbidis Hallaisan, 1600 m, 13.08.1908, Taquet 878" (lectotype P!, designated here by Pimenov; isolectotypes C, barcode C10005756, E!, TI!).

= Bupleurum nipponicum Koso-Pol., 1913, Journ. Russe Bot. 1-2: 1, tab. 1.

Type: JAPAN. "Jecciu, Miesi-yama, Chaku-sansaiko, 08. 1889, Tschonoski [Sugawa] 2493" (holotype LE!).

= Bupleurum longeradiatum Turcz. f. elatium Koso-Pol., 1916, Bull. Soc. Imp. Naturalistes Moscou, s.n. 29: 60.

= Bupleurum longeradiatum Turcz. var. elatium (Koso-Pol.) H. Hara, 1954, Enum. Sperm. Jap. 3: 301.

$\equiv$ Bupleurum yezoense Nakai, 1930, Veg. Mt. Apoi: 30, 62.

= Bupleurum nipponicum Koso-Pol. var. yezoense (Nakai) H. Hara, 1933, J. Jap. Bot. 9: 515. in syn.

Type: JAPAN. "Mt. Apoi, prov. Hidaka [Hokkaido], 1928, Nakai" (holotype TI!).

= Bupleurum yokoyamae Miyabe et Tatew., 1938, Trans. Sapporo Nat. Hist. Soc. 15: 134, fig. 2.

Type: JAPAN. "[Hokkaido] Yezo, prov. Tokachi, the upper Kogawa, Toyokoro, 15. 08. 1937, Yokoyama" (holotype SAP).
= Bupleurum shikotanense M. Hiroe, 1952, Acta Phytotax. Geobot. 14: 145.

$=$ Bupleurum longeradiatum Turcz. var. shikotanense (M. Hiroe) Ohwi, 1953, Bull. Natl. Sci. Mus. 33: 80.

$=$ Bupleurum longeradiatum Turcz. subsp. shikotanense (M. Hiroe) Vorosch., 1985, in Skvorzov (ed.), Florist. issl. v razl. raionakh SSSR: 183.

Type: RUSSIA. "Kuriles, Insl. Shikotan [Shakotan-Kagenama]: inter Shikotan et Kagenoma, 31. 08. 1931, Ohwi 1185" (holotype KYO!).

$=$ Bupleurum longeradiatum Turcz. var. pseudonipponicum Kitag., 1963, J. Jap. Bot. 38(10): 306.

Type: JAPAN. "Prov. Kai: circa Kusa-suberi in mt. Kita-dake, 23. 07. 1962, Ohba" (holotype TI!).

= Bupleurum quadriradiatum Kitag., 1964, J. Jap. Bot. 39(8): 252.

Type: JAPAN. "Japonia: prov. Ise: ad ripas prope Hatta-mura, Inabe, 25. 07. 1926, Usami” (holotype MAK).

Distribution in China: NW: Gansu; Tibet: Qinghai; N: Neimenggu A. R., Ningxia Huizu A. R., Shaanxi, Hebei, Shandong; NE: Heilongjiang, Jilin, Liaoning; Centr.: Henan, Anhui, Hubei, Jiangxi, Zhejiang.

Distribution outside China: Russia, Japan, Korea.

Bupleurum longeradiatum Turcz. f. australe Shan Renhwa et Li Yin, 1974, Acta Phytotax. Sin. 12(3): 269.

Type: CHINA. "Zhejiang, Tianmushan, Tan Changchi et Yue Yinsan 1282" (holotype NAS).

Distribution in China: Centr.: Anhui, Jiangxi, Zhejiang. Endemic.

Bupleurum longicaule Wall. ex DC., 1830, Prodr. 4: 131.

Type: NEPAL. "India orientalis, Gossain Than [Napolia] 1829, Wallich [557.1]" (lectotype G-DC!, barcode G00367572, designated here; isolectotypes BM!, barcode BM000944734, C!, CAL, E!, barcode E00000343, K!, K-WALLICH!, barcodes K000657114, K000657115, KW001000568, K001111268, K001111269, LE!, M, P!, barcode P00752895).

Distribution in China: NW: Gansu; Tibet: Xizang A. R., Qinghai; N: Ningxia Huizu A. R., Shaanxi, Shanxi; Centr.: Henan, Hubei; SW: Sichuan, Yunnan.

Distribution outside China: Nepal, India, Pakistan. 
Bupleurum longifolium L. subsp. aureum (Fisch. ex Hoffm.) Soó, 1966, Acta Bot. Acad. Sci. Hung. 12(1-2): 116.

$\equiv$ Bupleurum aureum Fisch. ex Hoffm., 1814, Gen. pl. Umbell.: 115.

$\equiv$ Diaphyllum aureum Hoffm., 1814, Gen. pl. Umbell., ed. 1: 115.

$\equiv$ Bupleurum longifolium L. var. aureum (Fisch. ex Hoffm.) H. Wolff, 1910, in Engler (ed.), Pflanzenreich 43(IV, 228): 52.

Type: Described on the basis of plants, cultivated in BG Gorenki. Herb. Hoffmann, 2252 (lectotype MW- Hoffm!, designated by Gubanov, 1993: 93; isolectotype LE!).

= Euphorbia perfoliata Scheutz, 1888, Kungl. Svenska Vetenskapsakad. Handl. 22: 10.

Type: RUSSIA. "Sibiria, Jenissej: Torgashina, Turczaninow" (holotype S).

= Bupleurum aureum Fisch, f. macranthum Kryl., 1903, Fl. Alt. Tomsk. Gub. 2: 504.

Type: RUSSIA. "[Altai] Mt. Tigeretzkiy Belok, in silvis coniferis, infra silvae terminum. 31. 07. 1891, Krylov" (lectotype TK, designated by Gureeva, Balashova, 2015: 5).

= Bupleurum longifolium L. subvar. longiinvolucratum H. Wolff, 1910, in Engler (ed.), Pflanzenreich 43(IV, 228): 53.

Type: RUSSIA. "Duhmberg, Fl. Altaic. N 760, 761, n.v."

= Bupleurum longifolium L. subvar. breviinvolucratum Trautv. ex H. Wolff, 1910, in Engler (ed.), Pflanzenreich 43(IV, 228): 53.

= Bupleurum aureum Fisch. ex Hoffm. var. breviinvolucratum (Trautv. ex H. Wolff) Shan Renhwa et Li Yin, 1974, Acta Phytotax. Sin. 12(3): 271.

= Bupleurum longifolium L. f. normale $\mathrm{H}$. Wolff, 1910, in Engler (ed.), Pflanzenreich 43(IV, 228): 53.

= Bupleurum longifolium L. f. angustatum $\mathrm{H}$. Wolff, 1910, in Engler (ed,), Pflanzenreich 43(IV, 228): 53.

= Bupleurum aureum Fisch. ex Hoffm. f. attenuatum Malysch., 1965, Flora Alp. Mont. Sajan. Orient.: 186.

Type: RUSSIA. "Ad fontes fluminis Uda, alt. 1600 m supra mare, in prato, 1961, Malyschev 998" (holotype IRK).

- Bupleurum longifolium auct. non L.: M. Hiroe, 1958, Umbell. Asia 1: 78.

Distribution in China: NW: Xinjiang Weiwuer A. R.

Distribution outside China: Russia, Mongolia, Kazakhstan, Kirghizia, Europe.
Bupleurum luxiense Li Yin et Pan Shengli, 1986, Acta Phytotax. Sin. 24(2): 150.

Type: CHINA. "Yunnan: Jian Shui, Lao Lidong, alt. 1800 m, 27. 08. 1983, Pan Shengli 0414" (holotype KUN!).

Distribution in China: SW: Yunnan. Endemic.

Bupleurum malconense Shan Renhwa et Li Yin, 1974, Acta Phytotax. Sin. 12(3): 284, tab. 58, fig. $1-9$.

Type: CHINA. "Sichuan: Markang, Erya, Mengbi Shan, in shrubs, alt. 2510 m, 26. 08. 1964, Yue Junsan, Song Guojie et Zhuang Tide 64020A" (holotype NAS).

$=$ Bupleurum sichuanense Pan Shengli et Hsu Pingsheng, 1992, SIDA Contr. Bot. 15(1): 91, fig. 1.

Type: CHINA. "Sichuan Province: Wenchuan District, alt. ca. $2000 \mathrm{~m}$ on open slope, margin of thickets, 21. 08. 1985, Pan Shengli 0442" (holotype $\mathrm{PE}$ ).

Distribution in China: NW: Gansu; Tibet: Xizang A. R., Qinghai; SW: Sichuan. Endemic.

Bupleurum marginatum DC., 1830, Prodr. 4: 132.

三 Bupleurum falcatum L. var. marginatum (DC.) C. B. Clarke, 1879, in Hooker f. (ed.), Fl. Brit. India 2: 676 .

$\equiv$ Bupleurum falcatum L. subsp. marginatum (DC.) C. B. Clarke ex H. Wolff, 1910, in Engler (ed.), Pflanzenreich 43(IV, 228): 133.

Type: INDIA/NEPAL. "Ad Kamaon et in Nepalia [1.Napolia. 1821. 2.Kamaon], 1821, Wallich, Blinkworth [556]" (lectotype G-DC!, designated here; isolectotypes BM!, barcode BM000622304, CAL, K!, K-WALLICH!, barcodes K000687091, K001111263, K001111265, K001111266, LE!).

= Bupleurum hoffmeisteri Klotzsch, 1862, in Klotzsch \& Garcke (eds.), Bot. Ergebn. Reise Waldemar: 147, tab. 52.

$=$ Bupleurum falcatum L. var. hoffmeisteri (Klotzsch) C. B. Clarke, 1879, in Hooker f. (ed.), Fl. Brit. India 2: 676.

Type: INDIA. "Im Himalaya, Hoffmeister" (holotype M?).

$=$ Bupleurum imaicolum Kerner, 1870, Ber. Naturw. Ver. Innsbruck 1(2): 115.

= Bupleurum falcatum L. var. imaicolum (Kerner) H. Wolff, 1910, in Engler (ed.), Pflanzenreich 43(IV, 228): 132.

Type: INDIA. "Himalaja; in ditione Lahul, in aridis montium lateribus, $10-14000$ ped. s. mare, Jaeschke" (holotype W). 
Distribution in China: NW: Gansu; Tibet: Xizang A. R., Qinghai; N: Ningxia Huizu A. R., Shaanxi, Shanxi; Centr.: Henan, Hubei, Jiangxi, Zhejiang; SW: Sichuan, Guizhou, Yunnan; S: Guangsi Zhuang A. R., Guangdong.

Distribution outside China: Myanmar, Bhutan, Nepal, India, Pakistan, Afghanistan.

Bupleurum marginatum DC. var. minutum Zhang Xiufu, 1995, Bull. Bot. Res., Harbin 15(4): 439.

Type: CHINA. "Shaanxi: Shenmudabaodang, in fixed sandyland, 12. 10. 1983, Yang Xiling 83157" (holotype LZD).

Distribution in China: N: Shaanxi. Endemic.

Bupleurum marginatum DC. var. stenophyllum (H. Wolff) Shan Renhwa et Li Yin, 1974, Acta Phytotax. Sin. 12(3): 292.

$\equiv$ Bupleurum falcatum L. var. stenophyllum $\mathrm{H}$. Wolff, 1910, in Engler (ed.), Pflanzenreich 43(IV, 228): 133.

$\equiv$ Bupleurum falcatum L. f. stenophyllum $(\mathrm{H}$. Wolff) P. K. Mukh., 1984, in B.D.Naithani, Fl. Chamoli 1: 255.

Type: INDIA. "Himal. Bor. Occ., Thomson".

Distribution in China: NW; Tibet: Xizang A. R.; Centr.: Hubei; SW: Sichuan, Yunnan.

Distribution outside China: Nepal, India.

Bupleurum microcephalum Diels, 1900, Bot. Jahrb. Syst. 29 (3-4) (Fl. Centr. China): 494.

Type: CHINA. "Setchuen, Tsaku lao: Wei kuan: Ta chai tzu, 17. 08. 1891, von Rosthorn 2529" (holotype $\mathrm{O}$ !).

= Bupleurum falcatum L. var. longepedunculatum H. Boissieu, 1902, Bull. Herb. Boissier (2 sér.) 2: 804, p.p.

Type: CHINA. "District de Tchen-Kéou-Tin (Su-Tchuen oriental), Farges" (syntype); "Chine occidentale. à Ta-Tsien-Lou, Mussot 162" (syntype P!); "Ta-Tsien-Lou, Pratt" (syntype); "Tongolo, princip. de Kia-La, 1893. Soulié 701" (syntypes MPU, barcodes MPU019123, MPU019124, P!).

Distribution in China: NW: Gansu; Tibet: Xizang A. R.; SW: Sichuan, Yunnan. Endemic.

Bupleurum multinerve DC., 1828, Mém. Soc. Phys. Genève 4: 500, nom. cons. prop.

Type: "Hortus Bot. Genev. - De graines envoyées en 1826 par M. Fischer, et qui avaient recuillies au monts Altai, 1828, 2581/6 ?" (syntype G-DC).

= Bupleurum striatum Steph. ex Roem. et Schult., 1820, Syst. Veg. (ed. 15) 6: 367. nom. rej. prop.
Type: "In Siberia. Stephan" (holotype B-Willd).

= Bupleurum multinerve DC. var. angustius DC., 1828, Mém. Soc. Phys. Genève 4: 500.

Type: RUSSIA. "In altioribus ad flum. Ononen Dahuriae, Prescott" (holotype G-DC?).

= Bupleurum multinerve DC. var. minus DC., 1829, Mém. Soc. Phys. Geneve 4: 500.

Type: RUSSIA. "In montibus Altaicis, Fischer" (holotype G-DC?).

= Bupleurum nervosum Trev., 1831, Symb. Phytol. 1: 22.

= Bupleurum multinerve DC. var. major Ledeb., 1844, Fl. Ross. 2: 265.

= Bupleurum multinerve DC. var. minor Ledeb., 1844, Fl. Ross. 2: 265.

= Bupleurum longeinvolucratum Krylov, 1903, Trudy Imp. S.-Peterburgsk. Bot. Sada 21(1): 17.

Type: RUSSIA. "In jugo Tigeretzki-belok montium Altaicorum occidentalium, in declivibus saxosis, infra limitem sylvarum, 31. 07. 1891, Krylov" (lectotype TK!, designated by Polozhij, Balashova (1989: 26), as "type"; isolectotype LE!).

- Bupleurum ranunculoides auct. non L.: Ledeb., 1829, Fl. Altaic. 1: 347.

Distribution in China: NW: Xinjiang Weiwuer A. R.

Distribution outside China: Russia, Mongolia, Kazakhstan, Europe.

Note: Chubarov (2003: 301) treated $B$. longeinvolucratum as a separate local microspecies.

Bupleurum petiolulatum Franch., 1894, Bull. Annuel Soc. Philom. Paris (sér. 8) 6: 117.

Type: CHINA. "Yun-nan, in silvis ad Maeul-chan supra Gnou-kay, 06. 08. 1889, Delavay" (syntype P!, barcode P00752944); "Yun-nan, in silvis ad Kou-toui supra Mo-so-yn, 17. 07. 1889, Delavay 4511" (lectotype P!, barcodes P00752943, P00752945, P00752946; designated here).

= Bupleurum longicaule Wall. ex DC. var. tibetanicum H. Wolff, 1910, in Engler (ed.), Pflanzenreich 43(IV, 228): 124.

Type: CHINA. "Ost-Tibet: Tongolo, Soulié 2600” (syntype P?); "Ost-Tibet: Ta-Tsien-Lu, Soulié 2171" (syntype P?).

Distribution in China: NW: Gansu; Tibet: Xizang A. R., Qinghai; SW: Sichuan, Yunnan. Endemic.

Bupleurum polyclonum Li Yin et Pan Shengli, 1984, Acta Phytotax. Sin. 22(2): 133, fig. 2.

Type: CHINA. "Yunnan: Huize, alt. 2200 m, 13. 08. 1980, Shanghai Prim. School 080119" (holotype SHMU; isotype KUN!). 
Distribution in China: SW: Yunnan. Endemic.

Bupleurum qinghaiense Li Yin et J. X. Guo, 1993, J. Chin. Pharm. Sci. 2(1): 40.

Distribution in China: Tibet: Qinghai. Endemic.

Bupleurum regelii Lincz. et V. M. Vinogr., 1985, in Novosti Sist. Vyssh. Rast. 22: 192, fig. 1.

Type: CHINA. "Maralty, Kunges, 8000', 08. 1879, Regel" (holotype LE!).

Distribution in China: NW: Xinjiang Weiwuer A. R. Endemic.

Bupleurum rockii H. Wolff, 1929, Repert. Spec. Nov. Regni Veg. 27: 186.

Type: CHINA. "Yangtze watershed, prefectural district of Likiang, eastern slopes of Likiang Snow range, 9-12000', 22. 08. 1922, Rock 6009" (holotype ?; isotype US).

= Bupleurum handelii H. Wolff, 1933, in Hand.Mazz., Symb. Sin. 7(3): 712.

Type: CHINA. "NW-Yunnan: Bei Lidjiang (Likiang), 1914-1916, Handel-Mazzetti 3855" (lectotype WU!, barcode WU0025979, designated here; isolectotype $\mathrm{W}$ !).

Distribution in China: Tibet: Xizang A. R.; SW: Sichuan, Yunnan. Endemic.

Bupleurum scorzonerifolium Willd., 1809, Enum. Pl. Horti Berol.: 300.

$\equiv$ Bupleurum falcatum L. var. scorzonerifolium (Willd.) Ledeb., 1844, Fl. Ross. 2: 267.

$\equiv$ Bupleurum falcatum L. subsp. scorzonerifolium (Willd.) Koso-Pol., 1915, Trudy Imp. S.-Peterburgsk. Bot. Sada 30(2) (Epitome Bupleur. Ross.): 219.

Type: RUSSIA. "Ad lacum Baical. 08. 1818" (lectotype B-Willd!, barcode B-W 05637-01 0, designated here by Pimenov; isolectotype LE!, barcode LE00015719).

= Bupleurum vanheurckii Müll.Arg., 1871, in Van Heurck (ed.), Obs. Bot. 2: 207.

$=$ Bupleurum chinense DC. f. vanheurckii (Müll. Arg.) Shan Renhwa et Li Yin, 1974, Acta Phytotax. Sin. 12(3): 293.

Type: CHINA. "Prope Chefoo in imperio China".

= Bupleurum falcatum L. var. angustissimum Franch., 1883, Ann. Sci. Nat. Paris (sér. 7) 16: 18.

= Bupleurum scorzonerifolium Willd. subsp. angustissimum (Franch.) Kitag., 1940, Rep. Inst. Sci. Res. Manchoukuo 4(7): 105.

= Bupleurum angustissimum (Franch.) Kitag., 1947, J. Jap. Bot. 21: 97.
= Bupleurum scorzonerifolium Willd. var. angustissimum (Franch.) Huang Yhuei, 1977, Fl. Pl. Herb. Chin. Bor.-Or. 6: 197.

Type: CHINA. "Mongolie: Oulachan, dans les hautes prairies arides, 07. 1856, David 2850" (holotype P!, barcode P00752921).

= Bupleurum angustissimum (Franch.) Kitag. f. latium Kitag., 1947, J. Jap. Bot. 21: 97.

Type: CHINA. "Manshuria: prov. Je-he: circa Ling-yuan, 13. 08. 1933, Nakai, Honda et Kitagawa" (holotype TI!).

= Bupleurum sinensium Gand., 1918, Bull. Soc. Bot. France 65: 30.

Type: CHINA. "China orientalis, ad Tche-Fou, Dedeaux" (holotype P?).

= Bupleurum falcatum L. var. chiliosciadium $\mathrm{H}$. Wolff, 1926, Acta Horti Gothob. 2: 303.

$=$ Bupleurum chinense DC. f. chiliosciadium $(\mathrm{H}$. Wolff) Shan Renhwa et Li Yin, 1974, Acta Phytotax. Sin. 12(3): 293.

Type: CHINA. "Chili: Hsiao-wu-tai-shan, Tienlin-ssu, unterhalb des Temples auf Loess, ca. 1600 m, 21. 09. 1921, H. Smith 176" (lectotype UPS!, designated here by Pimenov; isolectotypes BM!, barcode BM000793390, GB!, LD!, MO!, P!, PE!, $\mathrm{S} !)$.

= Bupleurum falcatum L. var. komarowii $\mathrm{H}$. Hara, 1974, Enum. Sperm. Jap. 3: 300.

- Bupleurum falcatum auct. non L.: Miq., 1867, Ann. Mus. Bot. Lugduno-Batavi 3: 246.

Distribution in China: NW: Gansu; Tibet: Qinghai; N: Neimenggu A. R., Ningxia Huizu A. R., Shaanxi, Shanxi, Hebei, Shandong; NE: Heilongjiang, Jilin, Liaoning; Centr.: Henan, Jiangsu, Anhui, Hubei, Zhejiang, Fujiang; SW: Sichuan; S: Guangxi Zhuang A. R.

Distribution outside China: Russia, Japan, Korea, Mongolia.

Bupleurum scorzonerifolium Willd. var. gracilius Kitag., 1940, Rep. Inst. Sci. Res. Manchoukuo 4(7): 105.

Type: CHINA. "Manshuria: Prov. Pin-chiang, environs of Harbin, 10. 08. 1936, Skvortzow 30" (syntype); "Duitschinshan station, steppe, 10. 07. 1927, Skvortzow 1931" (syntype); "Mangow station, steppe, 15. 07. 1923, Skvortzow 1932" (syntype); "[Manshuria], prov. Hsian-an orient.: Halasu station, mountain steppe, 10. 08. 1929, Skvortzow 1421, 1650" (syntypes).

Distribution in China: NE China. Endemic.

Bupleurum scorzonerifolium Willd. var. stenophyllum Nakai, 1937, J. Jap. Bot. 13(7): 487. 
इBupleurum stenophyllum (Nakai) Kitag., 1947, J. Jap. Bot. 21: 99.

Type: EASTERN ASIA. "Syntypi pluri ex Japonia, Korea et China" (see: Yi U Ch'ul, 2008: 263).

= Bupleurum stenophyllum (Nakai) Kitag. var. leiocarpum He Xingjin et Wang Changbao, 2011, J. Syst. Evol. 49(6): 586, fig. 12, B, b.

Type: CHINA. "NW Sichuan, Lixian, 22. 09. 2009. Ma mxg09092201" (holotype SZ).

Distribution in China: N: Hebei; SW: Sichuan.

Distribution outside China: Japan, Korea.

Bupleurum scorzonerifolium Willd. f. ensifolium (H. Wolff) Nakai, 1937, J. Jap. Bot. 13(7): 485.

$\equiv$ Bupleurum falcatum L. f. ensifolium $\mathrm{H}$. Wolff, 1910, in Engler ed.), Pflanzenreich 43(IV, 228): 133.

Type: JAPAN. Fig. 40 in J. Jiinouma (1874), SoMokou-Zoussets, ed. 2.

Distribution in China: Centr.: Hubei.

Distribution outside China: Japan, Korea.

Bupleurum scorzonerifolium Willd. $\mathrm{f}$. longiradiatum Shan Renhwa et Li Yin, 1974, Acta Phytotax. Sin. 12(3): 282.

Type: CHINA. "Hebei, Fengkuo cliff, Yabe" (holotype NAS).

Distribution in China: Centr.: Hubei. Endemic.

Bupleurum scorzonerifolium Willd. $\mathrm{f}$. pauciflorum Shan Renhwa et Li Yin, 1974, Acta Phytotax. Sin. 12(3): 282.

Type: CHINA. "Jiangsu, Jiangpu, Liu Shoulu et al., 1664A" (holotype NAS).

Distribution in China: Centr.: Jiangsu. Endemic.

Bupleurum sikiangense He Xingjin et Wang Changbao, 2011, J. Syst. Evol. 49(6): 586, fig. 19.

Type: CHINA. "E Xizang: Zuogong, 19. 09. 2006, Yu XZ 0014" (holotype SZ).

Distribution in China: Tibet: Xizang A. R. Endemic.

Bupleurum smithii H. Wolff, 1926, Acta Horti Gothob. 2: 304.

Type: CHINA. "Prov. Chili: Hsiao-wu-tai-shan, Yang-kia-p'ing, Tung-lin auf Wiesen, ca. $2000 \mathrm{~m}$, in prato granimoso, täml. torr. gräsäng, 05.09.1921, H. Smith 200" (lectotype UPS!, designated here); ibid., "06.09.1921, H. Smith 1117b" (syntype UPS!); "Prov. Chili: Hsiao-wu-tai-shan: Yang-kia-p'ing: Hsi-lin, auf alpinen und subalpinen Wiesen, 1600-
$2500 \mathrm{~m}$, in prato alpino, 01. 09. 1921, H. Smith 119, 208, 1161, 1074" (syntypes BM!, barcode BM000885308, GB!, barcode GB-0048806), LD!, PE!, UPS!).

= Bupleurum borealisinense Nakai, 1939, J. Jap. Bot. 15: 739 .

Type: CHINA. "Prov. Hopeh: in monte Hsiaowu-tai-shan, 05. 1914, Nagai 29, 206" (lectotype TI!, designated here); "Prov. Hopeh: in monte Hsiao-wu-tai-shan, 08. 1938, Tatenaka 85" (syntype $\mathrm{TI}$ ).

Distribution in China: NW: Gansu; Tibet: Xizang, Qinghai; N: Neimenggu A. R., Ningxia Huizu A. R., Shaanxi, Shanxi, Hebei; Centr.: Henan; SW: Sichuan. Endemic.

Bupleurum smithii $\mathrm{H}$. Wolff var. auriculatum Shan Renhwa et Li Yin, 1974, Acta Phytotax. Sin. 12(3): 273.

Type: CHINA. "Shanxi, Ninwu, 2100-2400 m, 11. 09. 1953, Shanxi Team 174" (holotype PE!).

Distribution in China: N: Shanxi. Endemic.

Bupleurum tenerum (Shan Renhwa et Li Yin) He Xingjin et Wang Changbao, 2011, J. Syst. Evol. 49(6): 583, fig. 4, A,a.

$\equiv$ Bupleurum petiolatum Franch. var. tenerum Shan Renhwa et Li Yin, 1974, Acta Phytotax. Sin. 12(3): 277, "petiolulatum".

Type: CHINA. "Sichuan, Mt. Emei, Shuo Jin Temple, Cheng Zeyun et Zhang Hongfu 23722" (holotype NAS; isotypes PE, SZ).

Distribution in China: NW: Tibet: Xizang A. R.; SW: Sichuan. Endemic.

Bupleurum thianschanicum Freyn, 1900, Mém. Herb. Boissier 13: 23.

Type: KAZAKHSTAN. "Turkestania orientalis, Thian-Schan, in valle fluvii Bajangol regionis silvatica superiore, 29. 07. 1896, Brotherus 753" (lectotype BRNM!, designated by Pimenov, Sutorý (2014: 1105); isolectotypes G!, barcode G00367640, $\mathrm{H}$, barcode H1394758).

Distribution in China: NW: Xinjiang Weiwuer A. R.

Distribution outside China: Kazakhstan, Kirghizia.

Bupleurum wenchuanense Shan Renhwa et $\mathrm{Li}$ Yin, 1974, Acta Phytotax. Sin. 12(3): 288, tab. 59.

Type: CHINA. "Sichuan, Wenchuan Xian, Yanmeng, 23. 08. 1958, Chen Shanyong, He Tao et Zhong Minfang 5664" (holotype SZ). 
Distribution in China: SW: Sichuan. Endemic.

Bupleurum yinchowense Shan Renhwa et $\mathrm{Li}$ Yin, 1974, Acta Phytotax. Sin. 12(3): 283, tab. 57, fig. 1-9.

Type: CHINA. "Shaanxi: Dingbian Xian, 08. 1963, Shaanxi Drug Control Inst." (holotype NAS).

Distribution in China: NW: Gansu; Tibet: Qinghai; N: Neimenggu A. R., Ningxia Huizu A. R., Shaanxi. Endemic.

Bupleurum yunnanense Franch., 1894, Bull. Annuel Soc. Philom. Paris (sér. 8) 6: 117.

Type: CHINA. "Yun-nan, in pascuis supra collum Yen-tze-hay, alt. 3200 m, 19. 07. 1887, Delavay 3066" (lectotype P!, barcode P00752806, designated here; isolectotypes A, barcode A00075430, CAS, barcode CAS0027444, GH, barcode GH00075431, $\mathrm{K}$ !, P!, barcode P00752805); "Yun-nan, in dumetis montis Tsang-chan supra Tali, alt. $2500 \mathrm{~m}, 20.08$. 1887, Delavay" (syntype P!).

Distribution in China: Tibet: Xizang A. R.; SW: Sichuan, Yunnan. Endemic.

Carlesia sinensis Dunn in Hooker, 1902, Icon. pl. 28: tab. 2739.

$\equiv$ Cuminum sinensis (Dunn) M. Hiroe, 1979, Umbelliferae World: 672.

Type: CHINA. "Chantung, frequent on rocks at 1000 to 2000 feet elevation in the Chefoo Mountains, 1889, Faber 234" (lectotype K!, barcode K000695726, designated here); "Chantung, Maingay" (syntype K!).

= Seseli taquetii H.Wolff, 1929, Repert. Spec. Nov. Regni Veg. 27: 188.

Type: KOREA. "Quelpaert: in petrosis Hallaisan, 13. 08. 1908, Taquet 890" (holotype E!, barcode E00265187).

Distribution in China: N: Neimenggu A. R., Hebei, Shandong; NE: Liaoning.

Distribution outside China: Korea.

Carum buriaticum Turcz. [ex Besser, Flora 17 (1, Beibl.): 13. 1834, nom. inval. (Art. 39.1)], 1844, Bull. Soc. Imp. Naturalistes Moscou 17: 713.

$\equiv$ Bunium buriaticum (Turcz.) Drude, 1898, in Engler \& Prantl (eds.), Nat. Pflanzenfam. 3(8): 194.

Type: RUSSIA. "In pratis transbaicalensibus passim copiosae provenit [In campis transbaicalensibus], 1829, Turczaninow" (lectotype LE!, designated by Vinogradova, 2005: 961; isolectotypes BP!, C!, H!, K!, KW!, M!, P, S).

= Carum furcatum H.Wolff, 1929, Repert. Spec. Nov. Regni Veg. 27: 187.
Type: CHINA. "Shansi: Chieh hesiuh Distr., Mien-Shan, Sung-lin-miao, ca $800 \mathrm{~m}$ s.m., in prato arido, 18. 06. 1924, H. Smith 5838" (lectotype UPS!, designated here; isolectotypes BM!, barcode BM000885491, PE!, S!, barcode S-G-1187).

= Carum pseudoburiaticum H.Wolff, 1930, Repert. Spec. Nov. Regni Veg. 27: 302.

Type: CHINA. "Prov. Shansi: Chiao-chang distr.: Pa-shui-ko, in prato arenoso ad rivulem ca 1900 m s.m., 21. 08. 1924, H. Smith 7103" (lectotype UPS!, designated here; isolectotypes BM!, barcode BM000885460, PE!, S!, barcode S-G-1188, TI!).

= Carum inodorum Siev., 1796, in Pall., Neue Nord. Beitr. 3: 229, nom. nud.

= Carum curvatum C. B. Clarke ex H. Wolff, 1929, Repert. Spec. Nov. Regni Veg. 27: 183, 187.

Type: CHINA. "Tibet, Gyantse, fields, 18.06.1907, Stewart" (lectotype K!, barcode K000685666, designated here; isolectotype CAL).

Distribution in China: NW: Xinjiang Weiwuer A. R., Gansu; Tibet: Xizang A. R., Qinghai; N: Neimenggu A. R., Ningxia Huizu A.R., Shaanxi, Shanxi, Hebei, Shandong; NE: Heilongjiang, Liaoning; Centr.: Henan, Hunan; SW: Sichuan.

Distribution outside China: Russia, Mongolia.

Carum buriaticum Turcz. f. angustissimum (Kitag.) Shan Renhwa et Pu Fating, 1977, Pl. Herb. Chin. Bor.-Or. 6: 205.

$\equiv$ Carum angustissimum Kitag., 1944, J. Jap. Bot. 20: 331.

Type: CHINA. "Manshuria: Prov. Hsing-an austr.: in pratis circa Wang-ye-miao, 25. 07. 1939, Kitagawa" (holotype TI!).

Distribution in China: NE. Endemic.

Carum carvi L., 1753, Sp. pl.: 263.

$\equiv$ Apium carvi (L.) Crantz, 1767, Stirp. Austr. 3: 101.

ESeseli carum Scop., 1771, Fl. Carniol. (ed. 2) 1: 215, nom. illeg. (Art. 52.1)

三Seseli carvi (L.) Spreng., 1820, in Roemer \&

Schultes (eds.), Syst. Veg. (ed. 15 bis) 6: 414.

三Sium carum Weber ex F.H.Wigg., 1780, Prim.

Fl. Holsat.: 24, nom. illeg. (Art. 52.1)

$\equiv$ Ligusticum carvi (L.) Roth, 1788, Tent. Fl.

Germ. 1: 124.

ECarum aromaticum Salisb., 1796, Prodr. Stirp.

Chap. Allerton: 169, nom. illeg. (Art. 52.1)

$\equiv$ Aegopodium carum Wibel, 1799, Prim. Fl.

Werth.: 199, nom. illeg. (Art. 52.1)

三Sium carvi (L.) Bernh., 1800, Syst. Verz.: 173.

$\equiv$ Bunium carvi (L.) M. Bieb., 1808, Fl. Taur.-

Caucas. 1: 211. 
$\equiv$ Foeniculum carvi (L.) Link, 1821, Enum. Hort.

Berol. Alt. 1: 284.

$\equiv$ Carum officinale Gray, 1821, Nat. Arr. Brit. P1.

2: 515, nom.illeg. (Art. 52.1)

$\equiv$ Pimpinella carvi (L.) Jess., 1879, Deutsche

Excursions-Fl.: 191.

$\equiv$ Carvi careum Bubani, 1900, Fl. Pyren. 2: 352.

$\equiv$ Selinum carvi (L.) E. H. L. Krause, 1904. in Sturm (ed.), Deutschl. Fl. (ed. 2) 12: 47, tab. 10.

Type: EUROPE. "In Europae borealis pratis. Herb. Clifford, 106, Carum 1" (lectotype BM-Cliff, designated by Reduron, Jarvis in Jarvis et al., 1993: 30).

$=$ Carum gracile Lindl., 1835, in Royle, Ill. Bot. Himal. Mts.: 232.

$=$ Carum carvi L. var. gracile (Lindl.) H. Wolff, 1926, Acta Horti Gothob. 2: 306.

= Carum carvi L. f. gracile (Lindl.) H. Wolff, 1927, in Engler (ed.), Pflanzenreich 90(IV, 228): 148.

Type: INDIA. "Nako in Kunawur, Royle" (lectotype CGE!, designated here; isolectotypes DD!, K!).

= Falcaria carvifolia C. A. Mey., 1844, Beitr. Pfl. Russl. 1: 14.

Type: EUROPE, RUSSIA. "In provinciae Tambov districtu Jelatomsk".

$=$ Carum polyphyllum Boiss. et Balansa, 1872, Fl. Orient. 2: 881.

Type: SYRIA. "In Syriâ interiori prope urbem Homs [Hums], 05. 1865, Post 243" (holotype G-BOIS!).

= Carum rosellum Woronow, Trudy Bot. Inst. Akad. Nauk SSSR (ser. 1 Fl. Sist. Vyssh. Rast.) 1: 218. 1933.

= Carum carvi $\mathrm{L}$. subsp. rosellum (Woronow) Vorosch., 1985, in Skvorzov (ed.), Florist. iss1. v razn. raionakh SSSR: 184.

Type: RUSSIA. "Prov. Kuban, distr. Batal., inter pag. Senibiskyi et Teberdinskyi, prata subalpina, 19. 07. 1921, Vvedensky 187" (holotype LE!).

= Ligusticum alatum (M. Bieb.) Spreng. var. pauciradiatum Parsa, 1948, Kew Bull. 1948: 200.

Type: IRAN. "N Persia, Kojur, 2800 m [Iran boreal, Kodjur], 16. 07. 1940, Parsa 703" (holotype $\mathrm{K}$ !).

Distribution in China: NW: Xinjiang Weiwuer A. R., Gansu; Tibet: Xizang A. R., Qinghai; N: Neimenggu A. R., Ningxia Huizu A.R., Shaanxi, Shanxi, Hebei, Shandong; NE: Heilongjiang; Centr.: Henan, Jiangxi; SW: Sichuan, Guizhou, Yunnan.

Distribution outside China: Russia, Myanmar, Bhutan, Nepal, India, Pakistan, Mongolia, Kazakh- stan, Kirghizia, Tajikistan, Uzbekistan, Afghanistan, Iran, Azerbaijan, Georgia, Armenia, Turkey, Iraq, Saudi Arabia, Syria. Europe, Africa, N. America (adv.), New Zealand (adv.), also cultivated in different countries.

Cenolophium denudatum (Fisch. ex Hornem.) Tutin, 1967, Feddes Repert. 74(1-2): 31, in obs.

$\equiv$ Athamanta denudata Fisch. ex Hornem., 1819, Hort. Hafn. Suppl.: 32.

$\equiv$ Selinum cenolophium E. H. L. Krause, 1904, in Sturm (ed.), Deutschl. Fl. (ed. 2) 12: 108. nom. illeg. (Art. 52.1).

Type: RUSSIA. "In Russia" (grown in Hortus Hafnensis from the seeds of Russian origin: In pratis circa fl. Irtysch et Buchtorma, coll. Ledebour and C. A. Meyer) (lectotype C!, barcode C0008381, designated here; isolectotypes $\mathrm{C}$ !, barcodes C0008378, C0008379, C0008380).

= Crithmum mediterraneum M. Bieb., 1819, Fl. Taur.-Caucas. 3: 215.

Type: "Ex hort. Gorenk. ex seminibus Sareptanis, 1817" (lectotype LE-Bieb!, designated by V. Bjalt in LE).

= Angelica fischeri Spreng., 1820, in Roemer \& Schultes (eds.). Syst. veg. (ed. 15 bis) 6: 605.

= Ligusticum fischeri (Spreng.) Link, 1821, Enum. Hort. Berol. Alt. 1: 276.

$=$ Cenolophium fischeri (Spreng.) W. D. J. Koch, 1824, Nova Acta Phys.-Med. Acad. Caes. Leop.Carol. Nat. Cur. 12(1): 103.

= Cnidium fischeri (Spreng.) Spreng., 1824, Syst. Veg. (ed. 16) 1: 888.

Type: EUROPE, RUSSIA. “Ad Volgam”.

= Ligusticum divaricatum Ledeb., 1824, Ind.

Sem. Hort. Dorpat.: 5.

= Cnidium divaricatum (Ledeb.) Ledeb., 1829, Fl. Altaic. 1: 324.

= Cenolophium divaricatum (Ledeb.) Besser, 1832, Flora 15(2 Beibl.): 27.

Type (probable type material): RUSSIA. "In Sibiria altaica. [Ligusticum divaricatum Ledeb.! M. Fisch. nom.: Athamanta denudata? altaica]" (LE!).

= Silaus longifolius Ledeb., 1829, Fl. Altaic. 1: 323, nom. illeg. (Art. 52.1).

Type (probable type material): RUSSIA. "In pratis circa fl. Irtysch et Buchtorma (L., M.) [Herb. Ledebour 247]" (LE!).

Distribution in China: NW: Xinjiang Weiwuer A. R.

Distribution outside China: Russia, Mongolia, Kazakhstan, Europe. 
Centella asiatica (L.) Urb., 1879, in Martius, Fl. bras. 11(1): 287, tab. 78 , fig. 1 .

$\equiv$ Hydrocotyle asiatica L., 1753, Sp. pl.: 234.

$\equiv$ Glyceria asiatica (L.) Nutt., 1818, Gen. N. Amer. P1. 1: 177.

$\equiv$ Chondrocarpus asiaticus (L.) Nutt., 1818, Gen. N. Amer. Pl. 2: corrigend.

Type: INDIA. "332.5" (lectotype LINN, designated by Tardieu-Blot, 1967: 25).

$=$ Hydrocotyle lunata Lam., 1783, in Lamarck et al. (eds.), Encycl. 1(1): 152.

$=$ Hydrocotyle asiatica L. var. lunata (Lam.) Pers., 1805, Syn. P1. 1: 302.

Type: INDIA. "L'Inde, Sonnerat".

$=$ Trisanthus cochinchinensis Lour., 1790, Fl. Cochinch. 1: 176.

Type: VIETNAM/CHINA. "Habitat frequenter ad sepes in Cochinchina, non cultis, si bene memini, etiam in China, et in aliis Indiae locis, Loureiro" (holotype $\mathrm{P}$; isotype $\mathrm{BM}$ !).

$=$ Hydrocotyle asiatica L. var. subrepanda Blume, 1826, Bijdr. Fl. Ned. Ind. 15: 883.

Type: INDONESIA. "Circa Bataviam in ruderatis et incultis", Blume?.

= Hydrocotyle hebecarpa DC., 1830, Prodr. 4: 63.

$=$ Hydrocotyle asiatica L. var. hebecarpa (DC.) Hassk., 1848, Pl. Jav. Rar.: 459.

Type: INDONESIA. "In insulâ Timor" (type P).

$=$ Hydrocotyle asiatica L. var. pedunculata Kuntze, 1891,Revis. Gen. P1. 1: 268.

Type: INDONESIA. "Java: Sindanglaja".

$=$ Hydrocotyle asiatica $\mathrm{L}$. var. subsessilis Kuntze, 1891, Revis. Gen. Pl. 1: 268.

Type: CHINA. "Hongkong, 1875, Kuntze" (holotype NY).

$=$ Centella coriacea Nannf., 1924, Svensk Bot. Tidskr. 18: 416, tab. 7, fig. 1, 2.

Type: S. AFRICA. "Natal: Africa australis, in humid pr. Durban, 15 m, 02. 07. 1893, Schlechter 2854" (holotype S).

= Centella boninensis Nakai ex Tuyama, 1936, Bot. Mag. (Tokyo) 50: 31, fig. 23.

$=$ Centella asiatica (L.) Urb. var. boninensis (Nakai et Tuyama) H. Hara, 1954, Enum. Sperm. Jap. 3: 304.

Type: JAPAN. "Bonin: Ins. Titizima: Suzaki, 15. 06. 1913, Nisimura 173" (holotype TI!).

Distribution in China: Tibet: Xizang A. R., Qinghai; N: Shaanxi; Centr.: Henan, Jiangsu, Anhui, Hubei, Hunan, Jiangxi, Zhejiang, Fujiang; SW: Sichuan, Guizhou, Yunnan; S: Taiwan, Guangxi Zhuang A.R., Guangdong, Hainan Dao.
Distribution outside China: Japan, Korea, Philippines, Papua-New Guinea, Indonesia, Malaysia, Vietnam, Laos, Thailand, Myanmar, Bangladesh, Bhutan, Nepal, India, Sri-Lanka, Pakistan, Iran, Georgia, Saudi Arabia, Yemen. Africa, N. America, S. America, Australia and Oceania.

Chaerophyllopsis huai H. Boissieu, 1909, Bull. Soc. Bot. France 56: 353.

Type: CHINA. "Yunnan (Chine) Vé gué mo dja près Pin-Tchouan, 26. 07. 1906, Ducloux 4565" (holotype P!, barcode P00752730).

Distribution in China: Tibet: Xizang A. R.; SW: Yunnan. Endemic.

Chaerophyllum prescottii DC., 1830, Prodr. 4: 225.

$\equiv$ Anthriscus prescottii (DC.) Veesenm., 1854, Beitr. Pflanzenk. Russ. Reiches 9: 84.

$\equiv$ Chaerophyllum bulbosum L. subsp. prescottii (DC.) Nym., 1879, Consp. Fl. Eur. 2: 300.

Type: RUSSIA. "In Sibiria altaica, Schangin" (holotype G-DC!).

- Chaerophyllum bulbosum auct. non L.: Ledeb., 1829, Fl. Altaic. 1: 360.

Distribution in China: NW: Xinjiang Weiwuer A. R.

Distribution outside China: Russia, Kazakhstan, Uzbekistan, Armenia. Europe.

Chaerophyllum villosum Wall. ex DC., 1830, Prodr. 4: 225.

Type: NEPAL. "In Nepaliae monte Chandaghiry, 06. 1821, Wallich [558]" (lectotype G-DC!, designated here; isolectotypes CAL, K!, K-WALLICH!, barcodes K001111270, K001111271).

= Anthriscus boissieui H.Lév., 1914, Bull. Acad. Intern. Geogr. Bot. 24: 281.

Type: CHINA. "[Yunnan] Plaines, vallons de Tcho-Ho, 2250 m, 07. 1913, Maire" (holotype E!).

Distribution in China: Tibet: Xizang A. R.; SW: Sichuan, Guizhou, Yunnan.

Distribution outside China: Bhutan, Nepal, India, Pakistan, Afghanistan.

Chamaesium delavayi (Franch.) Shan Renhwa et Liou Shoulu, 1979, in Shan Renhwa \& Sheh Menglan, Fl. Reipubl. Popularis Sin. 55(1): 130, tab. 64, fig. 1-5.

$\equiv$ Trachydium delavayi Franch., 1894, Bull. Annuel Soc. Philom. Paris (sér. 8) 6: 110. 
Type: CHINA. "Yunnan, ad colles calcareas montis Ma-eul-chan, haud procul a cacumine, alt. 3500 m, 10. 09. 1889, Delavay 3911" (lectotype P!, barcode P00834660, designated here; isolectotypes A, barcode A00062642, GH!, barcode GH00062643, K!, barcodes K000697367, K000980124, L!, NY, barcode NY00406294, P, barcodes P00245415, P04142109); "Yunnan, ad colles calcareis montis Ma-eul-chan, 3500 m, 06. 08. 1889, Delavay 4091" (syntype P!, barcode P00245416); "Province du Yunnan, pelouses des hautes montagnes; montagne Koua-la-po (Hokin), à 3000 m, 26. 08. 1884, Delavay 952" (syntype P!, barcode P00245413).

Distribution in China: SW: Sichuan, Yunnan. Endemic.

Chamaesium mallaeanum Farille et S. B. Malla, 1985, Candollea 40(2): 536, fig. 8 (1-5).

$\equiv$ Dolpojestella mallaeana (Farille et S. B. Malla) Farille et Lachard, 2002, Acta Bot. Gallica 149(4): 370.

Type: NEPAL. 'C. Nepal: massif de l'Annapurna, versant nord du col de Namun (Langtang himal) vers $3800 \mathrm{~m}$, dans la fruticée-mégaphobiée subalpine, 24. 09. 1981, Farille 81537" (holotype KATH; isotypes E!, barcode E00000248, G, JAY, P).

Distribution in China: Tibet: Xizang A. R.

Distribution outside China: Nepal.

Chamaesium novem-jugum (C. B. Clarke) C. Norman, 1938, J. Bot. 76: 231.

$\equiv$ Trachydium novem-jugum C. B. Clarke, 1879, in Hooker f. (ed.), Fl. Brit. India 2: 672.

$\equiv$ Aulacospermum novem-jugum (C. B. Clarke) Kachroo, Naqshi et U. Dhar, 1995, in Kachroo (ed.), Central Asia \& Kashmir Himal. archeobot. \& flor: 107, nom. inval., sine basion. (Art. 33.4)

Type: INDIA. "Sikkim: Lama Kangra, $14000 \mathrm{ft}$., 24. 07. 1849, Hooker Trachydium N 5" (lectotype K!, barcode K000697397, designated by M. F. Watson, 1998: 373; isolectotypes E, barcode E00018025, FI!, G, barcode G00367385, GH, barcode GH00075675, K!, barcode K000697398, M, barcode M0172833, MH!, NY, barcode NY00406297, P!); "Sikkim: Tungu. Hooker" (syntype K); "Sikkim: Yeumtong, Hooker" (syntypes K!, P!).

Distribution in China: Tibet: Xizang A. R.; SW: Yunnan.

Distribution outside China: Bhutan, Nepal, India.

Chamaesium paradoxum H. Wolff, 1925, Notizbl. Bot. Gart. Berlin-Dahlem 9: 275. $\equiv$ Trachydium paradoxum (H. Wolff) M. Hiroe, 1979, Umbelliferae World: 714.

Type: CHINA. "Tibetia orientalis: Tongolo, 21. 07. 1894, Soulié 2621, 2612" (lectotype BM!, designated here).

$=$ Trachydium novemjugum C. B. Clarke var. tongolense H. Boissieu, 1902, Bull. Herb. Boissier, (2 sér.) 2: 802.

Type: CHINA. "Thibet oriental, Ta-Tsien-Lou, Tongolo, 1893, Soulié 705 [619]" (lectotype P!, designated here; isolectotype $\mathrm{K}$ !).

Distribution in China: Tibet: Xizang A. R., Qinghai; SW: Sichuan, Yunnan. Endemic.

Chamaesium spatuliferum (W. W. Sm.) C. Norman, 1938, J. Bot. 76: 231.

三 Trachydium spatuliferum W. W. Sm., 1915. Notes Roy. Bot. Gard. Edinburgh 8: 210.

Type: CHINA. "Open stony pasture on the eastern flank of the Lichang Range. Lat. 27 ${ }^{\circ} 30^{\prime}$ N. Alt. 11000-12000 ft. 07. 1910, Forrest 6019" (lectotype E!, barcode E00000262, designated here; isolectotypes BM!, E!, K!, barcode K001091335, P!, barcode P00834620, US).

Distribution in China: Tibet: Xizang A. R.; SW: Sichuan, Yunnan. Endemic.

Chamaesium spatuliferum (W. W. Sm.) C. Norman var. minor Shan Renhwa et Liou Shoulu in Shan Renhwa \& Sheh Menglan (eds.), 1979, Fl. Reipubl. Popularis Sin. 55(1): 125, 298.

Type: CHINA. "Szechuan, Garze, 17. 08. 1960, Kan 17-222" (holotype NAS).

Distribution in China: SW: Sichuan. Endemic.

Chamaesium thalictrifolium H. Wolff, 1926, Acta Horti Gothob. 2: 302.

$\equiv$ Trachydium thalictrifolium (H. Wolff) M. Hiroe, 1979, Umbelliferae World: 714.

Type: CHINA. "Sze-ch'uan bor.: westlich von Sung-pan, $3200 \mathrm{~m}$, auf kurzgrasigen Wiesen [in prato herboso], 09. 07. 1922, H. Smith 2501" (syntypes BM!, GB!, barcode GB0048809, UPS!, W!); "Sze-ch'uan bor.: Hsioeh-shan, auf feuchten Wiesen, $3600 \mathrm{~m}$. Ad fontem, 24. 07. 1922, H. Smith 3695" (lectotype UPS!, designated here); "Sze-ch'uan bor.: Huang-ch'en-kuan, in lichten Mischwalde, $3300 \mathrm{~m}$. In silva sparsa, herbosa, 19. 08. 1922, H. Smith 4041" (syntype UPS!).

Distribution in China: NW: Gansu; Tibet: Xizang A. R., Qinghai; SW: Sichuan, Yunnan. Endemic. 
Chamaesium viridiflorum (Franch.) H. Wolff ex

Shan Renhwa, 1937, Sinensia 8: 87.

$\equiv$ Trachydium viridiflorum Franch., 1894, Bull. Annuel Soc. Philom. Paris (sér. 8) 6: 111.

Type: CHINA. "Province du Yunnan, lieux ombrages des rochers près du sommet du Maeul-chan, 3500 m, 10. 09. 1889, Delavay 3900" (lectotype K!, barcode K001091336, designated by Mukherjee and Constance, 1993: 33; isolectotypes GH, P!, barcodes P00245465, P00834667); "Yunnan, in rupibus adumbrates prope cacumina montis Ma-eul-chan, alt. 3500 m, 06. 08. 1889, Delavay 4092" (syntype P).

$=$ Trachydium markgrafianum Fedde ex $\mathrm{H}$. Wolff, 1930, Repert. Spec. Nov. Regni Veg. 27: 304.

$=$ Chamaesium markgrafianum (Fedde ex $\mathrm{H}$. Wolff) C. Norman, 1938, J. Bot. 76: 231.

Type: CHINA. "Tibet: Glacier Lake Camp, $\mathrm{N} 28^{\circ}$, E $100^{\circ} 45^{\prime}, 13000-14000 \mathrm{ft}$., on north facing earth slopes, well clothed with vegetation and Rhododendron scrub, 29. 07. 1921, Kingdon-Ward 4662" (holotype E!).

Distribution in China: SW: Sichuan, Yunnan. Endemic.

Chamaesium wolffianum Fedde ex H. Wolff, 1930, Repert. Spec. Nov. Regni Veg. 27: 305.

$\equiv$ Trachydium yunnanense M. Hiroe, 1979, Umbelliferae World: 714.

Type: CHINA. "Yunnan, Kane Pass, MekongYangtze divide, $11000 \mathrm{ft}$., open stony pasture, 08. 1914, Forrest 13011" (lectotype E!, designated here; isolectotype PE!).

Distribution in China: SW: Yunnan. Endemic.

Changium smyrnioides H. Wolff, 1924, Repert. Spec. Nov. Regni Veg. 19: 315.

$\equiv$ Conopodium smyrnioides (H. Wolff) M. Hiroe, 1958, Umbell. Asia 1: 95.

Type: CHINA. "China orientalis, prov. Chekiang, distr. Huchao, Cheng Tsungsu 13".

Distribution in China: Centr.: Henan, Jiangsu, Anhui, Hubei, Jiangxi, Zhejiang. Endemic.

Chuanminshen violaceum Sheh Menglan et Shan Renhwa, 1980, Acta Phytotax. Sin. 18(1): 48, fig. 2.

Type: CHINA. "Sichuan: Jintang Xian [culta!]. D. F. Gao et R. S. Gao 76-0004" (holotype NAS!).

Distribution in China: Centr.: Hubei; SW: Sichuan. Endemic.

Cicuta virosa L., 1753, Sp. pl.: 255.

$\equiv$ Cicutaria aquatica Lam., 1779, Fl. franç. 3: 445, nom. illeg. (Art. 52.1).
三Sium cicuta Weber ex F.H.Wigg., 1780, Prim.

Fl. Holsat.: 24, nom. illeg. (Art. 52.1).

$\equiv$ Cicutaria virosa (L.) Delarbre, 1800, Fl. Auvergne (ed. 2): 415.

三Selinum virosum (L.) E. H. L. Krause, 1904, in Sturm (ed.), Deutschl. Fl. (ed. 2), 12: 44.

Type: EUROPE. "In palludibus Europae sterilibus Ammann 361.1" (lectotype LINN!, barcode LINN-NL 361-1, designated by Hedge, Lamond, 1972: 425).

$=$ Cicuta virosa L. var. latisecta Čelak., 1875, Prodr. Fl. Boehm.: 563.

Type: EUROPE. "[Bohmen] loco not notato".

= Cicuta sachalinensis Koidz., 1930, Fl. Symb. Or.-Asiat.: 44.

Type: RUSSIA. "Saghalin: in humidis Korsakoff. 09. 1908. Faurie 984" (lectotype P, designated here; isolectotype E!, barcode E00000444).

Distribution in China: NW: Xinjiang Weiwuer A. R., Gansu; Tibet: Qinghai; N: Neimenggu A. R., Shaanxi, Shanxi, Hebei, Shandong; NE: Heilongjiang, Jilin, Liaoning; Centr.: Henan, Jiangsu, Fujiang.

Distribution outside China: Russia, Japan, Korea, India, Mongolia, Kazakhstan, Kirghizia, Georgia, Turkey. Europe, Australia and Oceania.

Cnidium dauricum (Jacq.) Turcz. ex Fisch. et C. A. Mey. (eds.), 1835, Index Sem. Horti Petrop. 2: 33.

$\equiv$ Laserpitium dauricum Jacq., 1776, Hort. Vindob. 3: 22, tab. 38.

इLigusticum dauricum (Jacq.) Koso-Pol., 1916, Bull. Soc. Imp. Naturalistes Moscou, s.n. 29: 120, "dahuricum".

三Selinum dauricum (Jacq.) Leute, 1970, Ann. Naturhist. Mus. Wien 74: 508.

Type: RUSSIA. "Plants grown from seeds obtained from Dahuria" (holotype W?).

= Peucedanum selinoides DC., 1830, Prodr. 4: 181.

= Thysselinum involucratum Moench, 1794. Methodus: 85.

Type: "Culta in HB Lund («Semina inter alia Sibirica accepti») [Ex Sibiria. Stephan?]".

= Cnidium cuneatum Ledeb., 1829, Fl. Altaic. 1: 331.

= Aulacospermum cuneatum (Ledeb.) Ledeb., 1833, Fl. Altaic. 4: 335.

Type: RUSSIA. "[Altai] In pratis ad fl. Kerlyk, Herb. Ledeb. 428.2" (lectotype LE!, designated here; isolectotype P, barcode P03237350).

Distribution in China: N: Neimenggu A. R., Shanxi, Hebei; NE: Heilongjiang, Jilin. 
Distribution outside China: Russia, Korea, Mongolia.

Cnidium japonicum Miq., 1867, Ann. Mus. Bot. Lugduno-Batavi 3: 60; 1867. Prolus. Fl. Jap.: 248.

三Selinum japonicum (Miq.) Franch. et Sav., 1875, Enum. P1. Jap. 1: 186.

$\equiv$ Oenanthe japonica (Miq.) Nakai, 1922, in Mori, Enum. pl. Cor.: 272, nom. illeg., non Drude (1898).

Type: JAPAN. "In agris prope Nangasaki inter frumenta pierot [Insula Jesso, circa Hakodate], 1861, Albrecht" (syntypes LE!, NYBG); "Japan. Bürger" (syntype, probable lectotype L!, barcode L0366843); "Japan. Siebold" (syntypes L!, barcodes L0366846, L0366898, L0366899).

Distribution in China: N: Shandong; NE: Liaoning.

Distribution outside China: Japan, Korea.

Cnidium monnieri (L.) Cusson ex Jussieu, 1787, Mém. Soc. Nat. Med. Paris: 280.

三Selinum monnieri L., 1755, Cent. Pl. I: 9.; 1759, Amoenit. Acad. 4: 269.

$\equiv$ Cicuta monnieri (L.) Crantz, 1767, Class. Umbell. Emend.: 98.

$\equiv$ Selinum densiflorum Salisb., 1796, Prodr.

Stirp. Chap. Allerton: 162, nom. illeg. (Art. 52.1).

三 Pinasgelon monnieri (L.) Raf., 1840, Good

Book: 52, "monniera".

三 Ligusticum monnieri (L.) Calest., 1905,

Webbia 1: 211.

Type: EUROPE, FRANCE. "In Gallia australi. Monnier, 344.14" (lectotype LINN, designated by Watson and Reduron in Reduron, 2007: 864).

= Cicuta sinensis Zuccagni, 1806, Cent. Observ. Bot.: No 56.

Type: "Culta in H. B. Florentino".

= Cnidium microcarpum Turcz. ex Besser, 1834, Flora 17(1, Beibl.): 13, nom. illeg. (Art. 52.1).

Type: RUSSIA. "In salsis Dahuriae [In salsis prope ostium Onon-Borsa], 1831, Turczaninow" (lectotype MW!, designated here; isolectotypes FI!, K!, LE!, S!, barcode S12-231983).

= Seseli daucifolium C.B.Clarke, 1879, in Hooker f. (ed.), Fl. Brit. India 2: 693.

Type: INDIA. "East Bengal, from Bhotan and Assam to Dacca and Chittagong [Dhubari], 05. 04. 1800, Hamilton [7209]" (syntypes CAL, K!). BANGLADESH. "Dacca, 26.04.1868, Clarke 6878” (syntype K!, barcode K000685128).

Distribution in China: NW: Xinjiang Weiwuer A. R., Gansu; Tibet: Xizang A. R.; N: Neimenggu
A. R., Shaanxi, Shanxi, Hebei, Shandong; NE: Heilongjiang, Jilin, Liaoning; Centr.: Henan, Jiangsu, Anhui, Hubei, Hunan, Jiangxi, Zhejiang; SW: Sichuan, Guizhou, Yunnan; S: Taiwan, Guangxi Zhuang A.R., Guangdong, Hainan Dao.

Distribution outside China: Russia, Korea, Vietnam, Laos, Bangladesh, India, Mongolia, Europe, N. America.

Cnidium officinale Makino, 1907, in Iinuma (ed.), Somoku-Dzusetsu (ed. 3) 1(5): 345; 1908. Bot. Mag. (Tokyo) 22: 173.

三Ligusicum officinale (Makino) Kitag., 1963, J. Jap. Bot. 38(4): 108.

Distribution in China: China (cult.)

Distribution outside China: Japan (cult.), Korea (cult.). Origin is not clear.

Conioselinum acuminatum (Franch.) Lavrova, 2002, Abstr. Intern. Sci. Conf. Syst. Higher Pl. (Moscow): 66.

三Ligusticum acuminatum Franch., 1894, Bull. Annuel Soc. Philom. Paris (sér. 8) 6: 131.

三Ligusticopsis acuminata (Franch.) Leute, 1969, Ann. Naturhist. Mus. Wien 73: 69, tab. 3, fig. 1.

Type: CHINA. "Les bois de San tcha ho, au dessus de Mo-so-yn a 3000 m d'alt., 05. 09. 1887, Delavay 2945" (lectotype P!, barcode P03237428, designated by Leute, 1969: 69; isolectotypes GH, barcode GH00076823, K!, barcode K000685241; P, barcodes P0 3224576, P03237429, P03237430, P03237431).

= Ligusticum silvaticum H. Wolff, 1926, Acta Horti Gothob. 2: 315.

Type: CHINA. "Sze-ch'uan bor.: Vom Huangch'en-kuan, alt. 3100-3300 m, Längs stig i snårig ung lövskog, 19. 08. 1922, H. Smith 4040" (lectotype UPS!, designated here; isolectotypes GB!, barcode GB-0048815, W!).

Distribution in China: NW: Gansu; N: Shaanxi; Shanxi, Centr.: Henan, Anhui, Hubei, Hunan; SW: Sichuan, Yunnan.

Distribution outside China: Bhutan.

Conioselinum anthriscoides (H. Boissieu) Pimenov et Kljuykov, 2015, Skvortsovia 2(1): 33, figs. 1-2.

ECarum anthriscoides H. Boissieu, 1906, Bull. Soc. Bot. France 53: 426.

E Aegopodium anthriscoides (H. Boissieu) $\mathrm{H}$. Boissieu, 1909, Bull. Soc. Bot. France 56: 350.

Type: CHINA. "Su-tschuan oriental, district de Tcheu-keou-tin, Farges 119-bis" (lectotype P!, 
designated by Pimenov (Pimenov, Kljuykov, 2015: 33); isolectotypes UC!, B!, K!).

= Ligusticum sinense Oliv., 1891, in Hooker f., Icon. Pl. (ser. 3) 20: tab. 1958.

$=$ Conioselinum sinomedicum Pimenov et Kljuykov, 2003, Willdenowia 33(2): 367, fig. 19, nom. illeg. (Art. 52.1).

Type: CHINA. "Central China, prov. Hupeh, district Hsingshan, 1885-1888, Henry 6759B" (lectotype K!, designated by Pimenov (Pimenov, Kljuykov, 2015: 33); isolectotypes BM!, barcode BM000885474, E!, barcode E00265234, G!, GH, PE!, US!); "Prov. Szechwan, district NOWushan, Henry 6759A" (syntypes BM!, barcode BM000885475, G, barcode G00366993, GH, barcode GH00112224, K!, LE!, P, barcode P03221103).

= Ligusticum markgrafianum Fedde ex H. Wolff, 1930, Repert. Spec. Nov. Regni Veg. 27: 313.

Type: CHINA. "Prov. Hubei, Henry 4954" (lectotype K!, barcode K000685139, designated here; isolectotypes E!, barcode E00002344, US, barcode US00731134).

= Ligusticum pilgerianum Fedde ex H. Wolff, 1930, Repert. Spec. Nov. Regni Veg. 27: 322, nom. illeg. (Art. 53.1), non Ligusticum pilgerianum $\mathrm{H}$. Wolff (1930: 307).

= Ligusticum harrysmithii M. Hiroe, 1958, Umbell. Asia 1: 109.

Type: CHINA. "Shansi, Chiao-chung Distr., Pa-shui-ko-shan, in prato fruticoso herboso ad silvulam, $2000 \mathrm{~m}, 24.08 .1924$, H. Smith 7112" (lectotype UPS!, designated by Hiroe, 1958: 110; isolectotypes MO, barcode MO-345371, PE!, S!, TI!); "Southwrestern Kansu, Upper Tebbu country, in moist meadows and along the streams in Drakana, 10000', Rock 14590" (syntypes A, BM, barcode BM000885416, GH, barcode GH00076820).

= Ligusticum levistifolium H. Wolff, 1930, Repert. Spec. Nov. Regni Veg. 27: 323.

Type: CHINA. "Tibet: Tse-Kou [Tsekou, Yunnan], Manberg 85" (holotype K!, barcode K000685138).

$=$ Ligusticum sinense Oliv. var. alpinum Shan Renhwa ex Fu Kuntsun, 1981, Fl. Tsinling. 1(3): 461, 419.

Type: CHINA. "Shensi: Hwa-in Hsien, Hwayang Commune, Ta-pai-yang-cha, alt. 1400-1500 m, 23. 09. 1974, Fu Kuntsun 17254" (holotype WUK).

= Ligusticum sinense Oliv. var. hupehense Zhang Haidao, 1993,Acta Phytotax. Sin. 31(3): 281.

Type: CHINA. "Hubei, Dashanding, alt. 1600 $\mathrm{m}$, by the road side, in moist, 13. 09. 1984, Liu et
Lin 10" (holotype Wuhan Institute for Food and Drug Control).

Distribution in China: NW: Gansu; N: Neimenggu A. R., Shanxi, Shaanxi; Centr.: Henan, Hubei, Jiangxi; SW: Sichuan, Guizhou, Yunnan. Endemic.

Conioselinum morrisonense Hayata, 1921, Icon. Pl. formosan. 10: 20, fig. 12.

Type: CHINA. "[Taiwan] Monte Morrison, ad 10000 ped. alt., 25. 11. 1906, Mori 1849" (holotype TAIF).

Distribution in China: S: Taiwan. Endemic.

Conioselinum nematophyllum Pimenov et Kljuykov, 2003,Willdenowia 33(2): 361, fig. 8.

$\equiv$ Ligusticum filifolium Shan Renhwa et $\mathrm{Pu}$ Fating, 1991, Acta Phytotax. Sin. 29(6): 538, fig. 7, nom. inval., non Hook. f. (1852).

$\equiv$ Ligusticum nematophyllum (Pimenov et Kljuykov) Pu Fating et M. F. Watson, 2004, Acta Phytotax. Sin. 42(6): 564.

Type: CHINA. "Sichuan, Nanping, Gong-Galing, at the edge of alpinen conisilvae, alt. $3200 \mathrm{~m}$, 25. 08. 1984, He Jin 110" (holotype CDBI!).

Distribution in China: SW: Sichuan. Endemic.

Conioselinum pseudoangelica ( $\mathrm{H}$. Boissieu) Pimenov et Kljuykov, 2003, Willdenowia 33(2): 362, fig. 14.

$\equiv$ Ligusticum pseudoangelica $\mathrm{H}$. Boissieu, 1903, Bull. Herb. Boissier (2 sér.) 3(10): 845.

$\equiv$ Pleurospermum pseudoangelica (H. Boissieu)

H. Boissieu, 1906, Bull. Soc. Bot. France 53: 434.

Type: CHINA. "[Yunnan] Les marais de KanHay, sur le Hee chan men (Lan Kong), à $2800 \mathrm{~m}$ d'altit., 21.09.1885, Delavay 2021" (lectotype P!, barcode P05224888, designated by Leute, 1970: 494; isolectotype P, barcodes P03224910, P03224911).

$=$ Ligusticum glaucifolium H.Wolff, 1930, Repert.Spec. Nov. Regni Veg. 27: 312.

Type: CHINA. "Yunnan, Lat. 27²0', 10000'. Shady situations by streams on the Tong-Shan in the Yangtze bend, 09. 1913, Forrest 11280" (lectotype E!, barcode E00002345, designated here; isolectotypes BM!, barcodes BM000885414, BM000885414, K!, barcode K000695862, PE!).

= Ligusticum glaucifolium H. Wolff, 1930, Repert. Spec. Nov. Regni Veg. 27: 312.

Type: CHINA. "Yunnan, Lat. 27²0', 10000'. Shady situations by streams on the Tong-Shan in the Yangtze bend, 09. 1913, Forrest 11280" (lectotype E!, designated here; isolectotypes BM!, K!, PE!).

Distribution in China: SW: Yunnan. Endemic. 
Conioselinum pteridophyllum (Franch.)

Lavrova, 2002, Abstr. Intern. Sci. Conf. Syst. Higher Pl. (Moscow): 67.

三 Ligusticum pteridophyllum Franch., 1894, Bull. Annuel Soc. Philom. Paris (sér. 8) 6: 132.

$\equiv$ Ligusticopsis pteridophylla (Franch.) Leute, 1969, Ann. Naturhist. Mus. Wien 73: 78, tab. 5, fig. 4.

Type: CHINA. "Yunnan, in silvis ad collum Piiou-se supra Tapin-tze, alt. 2000 m, 15. 10. 1886, Delavay 2517" (syntypes A, barcode A00112221, P!); "Ad pedem montis Tsang-chan, supra Tali, 25. 09. 1888, Delavay 3618" (lectotype P!, barcode P03776960, designated by Pimenov et al., 2003: 362); isolectotypes P, barcodes P03776959, P03776961); “Ad basin montis Ma-eul-chan, 30. 10. 1889, Delavay” (syntypes K!, barcode K000685239, $\mathrm{P} !)$.

= Peucedanum reptans Diels, 1900, Bot. Jahrb. Syst. 29 (3-4) (Fl. Centr. China): 502.

= Ligusticum reptans (Diels) H. Wolff, 1926, Acta Horti Gothob. 2: 316.

Type: CHINA. "Setchuen, Nan ch'uan: Hei wan ai, 25. 09. 1891, von Rosthorn 1012" (holotype O!).

Distribution in China: NW: Gansu; Tibet: Xizang A. R.; N: Shanxi; SW: Sichuan, Guizhou, Yunnan. Endemic.

Conioselinum reflexum Pimenov et Kljuykov, 2003, Willdenowia 33(2): 365, fig. 12, 16.

Type: CHINA. "China, Yunnan, NW part, Zhongdian Co., $27 \mathrm{~km} \mathrm{~N}$ od Zhongdian. Lat. 28 $8^{\circ}$ ' N, Long. 9943' E. Alt. 3800 m, 26. 09. 1998, Pimenov, Kljuykov, Hu Zihao et Liu Qixing 436" (holotype MW!; isotype B!, barcode B 10 0058090).

Distribution in China: SW: Sichuan, Yunnan. Endemic.

Conioselinum shanii Pimenov et Kljuykov, 2003, Willdenowia 33(2): 365, fig. 13, 17.

$=$ Conioselinum chinense auct. non Britton et al.: Qiang Sheng, Fl. Anhui 3: 631, fig. 1677. 1988.

Type: CHINA. "Anhui, 11. 1990, 90133" (holotype NAS!).

Distribution in China: Centr.: Anhui, Jiangxi, Zhejiang. Endemic.

Conioselinum sinchianum ( $\mathrm{Fu}$ Kuntsun) Pimenov et Kljuykov, 2003, Willdenowia 33(2): 367, fig. 18.

$\equiv$ Cnidium sinchianum $\mathrm{Fu}$ Kuntsun, 1981, in Fl. Tsinling. 1(3): 459, 415, fig. 355. $\equiv$ Selinum sinchianum (Fu Kuntsun) Yuan Changchi et Li Libo, 1993, Acta Bot. Boreal.-Occid. Sin. 13(1): 66.

Type: CHINA. "Shensi: Feng Hsien, Sin-chashan, Paimu-tze-tan, alt. 2430-2450 m, 21. 09. 1951, Liou Kimou 10682" (holotype WUK; isotype KUN!).

$=$ Ligusticum moniliforme Peng Zexiang et Zhang Bingyan, 1995, Acta Phytotax. Sin. 33(3): 302.

Type: CHINA. "Gansu: Tianjiahe, near the village Lannigou, alt. $2300 \mathrm{~m}$, on borders of field, 18. 09. 1993, Zhang Bingyan 93060" (holotype LZU).

Distribution in China: NW: Gansu; Tibet: Qinghai; N: Shaanxi. Endemic.

Conioselinum smithii (H. Wolff) Pimenov et Kljuykov, 2003, Willdenowia 33(2): 369, fig. 20-22.

$\equiv$ Ligusticum smithii H. Wolff, 1926, Acta Horti Gothob. 2: 314.

Type: CHINA. "Chili: Hsiao-wu-tai-shan, Yangkia-p'ing, Hsi-lin, 1600-2600 m, 01. 09. 1921, H. Smith 1097" (holotype UPS!).

= Ligusticum longilobum H. Wolff, 1926, Acta Horti Gothob. 2: 313.

$=$ Ligusticopsis longiloba (H. Wolff) Leute, 1969, Ann. Naturhist. Mus. Wien 73: 78.

Type: CHINA. "Chili: Hsiao-wu-tai-shan, Tienlin-ssu, ca. 2300 m. In prato alpino, 18. 09. 1921, H. Smith 101" (holotype UPS!).

= Cnidium jeholense Nakai et Kitag., 1934, Rep. First Sci. Exped. Manchoukuo, sect. IV, 1: 38, tab. 12.

= Ligusticum jeholense (Nakai et Kitag.) Nakai et Kitag., 1936, Rep. First Sci. Exped. Manchoukuo, sect. IV, 4: 36, 90.

$=$ Tilingia jeholensis (Nakai et Kitag.) Leute, 1970, Ann. Naturhist. Mus. Wien 74: 511.

$=$ Conioselinum jeholense (Nakai et Kitag.) Pimenov, 1985, Vestnik Moskovsk. Gosud. Univ. (ser. 16, Biol.) 3: 71.

Type: CHINA. "In sylvis montis Wu-lin-shan, 02. 09. 1933, Nakai, Honda et Kitagawa" (holotype $\mathrm{TI}$ ).

= Ligusticum jeholense (Nakai et Kitag.) Nakai et Kitag. var. tenuisectum Chu Youchang, 1977, Fl. P1. Herb. Chin. Bor.-Or. 6: 293, 241, tab. 96, fig. 8.

Type: CHINA. "Liaoning, Benxi, 17. 09. 1963, Y. C. Teng 1263" (holotype IFP).

Distribution in China: N: Neimenggu A. R., Shanxi, Hebei, Shandong; NE: Jilin, Liaoning; Centr.: Henan. 


\section{Distribution outside China: Russia.}

Conioselinum tataricum Hoffm., 1816, Gen. pl. Umbell. (ed. 2): 185, fig. 5, in nota.

Type: Described on the basis of plants, cultivated in Botanical Garden Gorenki ("hort. Gorenk.").

= Conioselinum vaginatum Thell., 1926, in Hegi (ed.), Ill. Fl. Mitt.-Eur. 5(2): 1329, non Ligusticum vaginatum Spreng. (1815).

Type: RUSSIA. "In Sibiria".

= Selinum gmelinii Bray, 1818, Denkschr. Bot. Ges. Regensb. 1(2): 36.

Type: EUROPE, LITHUIANIA. "In fruticetis inter Corylos et alios frutices".

= Conioselinum fischeri Wimm. et Grab., 1827, Fl. Siles. 1: 266.

Type: EUROPE, POLAND. "[Livonia] In montanis herbosis. Auf dem Leitenberge u. Kleinberge im Gesenke".

= Conioselinum univittatum Turcz. [1838, Bull. Soc. Imp. Naturalistes Moscou: 93, nom. inval. (Art. 39.1)] ex Kar. et Kir., 1842, Bull. Soc. Imp. Naturalistes Moscou 15: 363.

= Selinum univittatum Turcz., 1844. Bull. Soc. Imp. Naturalistes Moscou 17: 735.

Type: RUSSIA. "In pratis sylvaticis ubique [Ircutia, 1828] Turczaninow 117" (lectotype LE!, designated by Vinogradova, 2005: 962; isolectotypes: $\mathrm{KW}$ !, LE!).

$=$ Conioselinum altaicum Rupr., 1859, Beitr. Pflanzenk. Russ. Reiches 11 (Revis. Umb. Kamtsch.): 22.

= Conioselinum latifolium Rupr., 1869, Mém. Acad. Imp. Sci. Saint-Petersbourg (ser. 7) 14(4) (Sert. Tiansch.): 48.

Type: KIRGHIZIA. "Suukty, 01. 08. 1867, Osten-Saken" (syntype LE?); "Molda-assuSchlucht, 12. 08. 1867, Osten-Saken" (lectotype LE!, designated here).

$=$ Selinum papyraceum C. B. Clarke, 1879 , in Hooker f. (ed.), Fl. Brit. India 2: 701.

$=$ Cortia papyracea (C. B. Clarke) Leute, 1969, Ann. Naturhist. Mus. Wien 73: 84.

$=$ Conioselinum papyraceum $(\mathrm{C} . \mathrm{B}$. Clarke) Pimenov et Kljuykov, 1999, Bot. Zhurn. (St. Perersburg) 84(3): 91, fig. 1, map 2.

Type: INDIA. "Polgam, Kashmir,12000 ft., 04. 09. 1875, Clarke 31047" (lectotype K!, designated by Nasir, 1972: 117).

= Conioselinum schugnanicum B. Fedtsch., 1902, Trav. Mus. Bot. Acad. Petersb. 1: 135.

Type: TAJIKISTAN. "In valle fl. Gunt prope Rivak, in frutices, 29. 07. 1901, B. A. Fedtschenko" (lectotype LE!, designated by Vinogradova, 1999: 84); "A castellum Chorog ad pag. Rivak, ad riguorum et rivulorum margines, in frutices, 29. 07. 1901, B. A. Fedtschenko" (syntype LE!); "In valle fl. Toguzbulak, a locum Vankala (in fl. Gunt) ad locum Dzhilandy, 21-31. 07. 1901, B. A. Fedtschenko" (syntype LE!).

Distribution in China: NW: Xinjiang Weiwuer A. R.

Distribution outside China: Russia, India, Pakistan, Mongolia, Kazakhstan, Kirghizia, Tajikistan, Uzbekistan, Afghanistan, Europe.

Conioselinum tenuisectum (H. Boissieu) Pimenov et Kljuykov, 2003, Willdenowia 33(2): 372, fig. 23-24.

$\equiv$ Ligusticum tenuisectum H. Boissieu, 1903, Bull. Herb. Boissier (2 sér.) 3(10): 843.

$\equiv$ Ligusticopsis tenuisecta (H. Boissieu) Leute, 1969, Ann. Naturhist. Mus. Wien 73: 79.

三 Yunnania tenuisecta (H. Boissieu) Lavrova, 2006, Doklady Timir'jazev Agrocult. Akad. 278: 31.

Type: CHINA. "Su-Tchuen oriental, district de Tchou-Kéou-Tin, Farges 119" (lectotype P!, barcode P03221073, designated here; isolectotypes K!, barcode K000685137, P, barcodes P03221075, P03221078, P03221079, P03221080).

= Cnidium nullivittatum $\mathrm{Fu}$ Kuntsun, 1981, Fl. Tsinling. 1(3): 460, 415, fig. 356.

$=$ Selinum nullivittatum (Fu Kuntsun) Yuan Changchi et Li Libo, 1993, Acta Bot. Boreal--Occid. Sin. 13(1): 66.

$=$ Ligusticum nullivittatum ( $\mathrm{Fu}$ Kuntsun) $\mathrm{Pu}$ Fating et M. F. Watson, 2004, Acta Phytotax. Sin. 42(6): 564.

Type: CHINA. "Shensi: Hwa-in Hsien, Ta-paiyang-cha, alt. 1600-1900 m, 27. 09. 1974, Fu Kuntsun 17241" (holotype WUK).

Distribution in China: N: Shaanxi, Hebei; Centr.: Henan, Hubei; SW: Sichuan, Yunnan. Endemic.

Conioselinum tenuissimum (Nakai) Pimenov et Kljuykov, 2003, Willdenowia 33(2): 373.

$\equiv$ Angelica tenuissima Nakai, 1919, Bot. Mag. (Tokyo) 33: 10.

इLigusticum tenuissimum (Nakai) Kitag., 1941, J. Jap. Bot. 17: 562.

Type: KOREA. "Corea media: in silvis montium Kumgangsan v. Diamond mountains, Uchiyama et Nakai, 5728-5729" (holotype TI!). 
- Ligusticum multifidum Nakai, 1909, Tyosensyokubutu (Fl. Koreana) 1: 265, nom. illeg. (Art. 53.1), non Sm. (1812).

Distribution in China: N: Hebei; NE: Liaoning. Distribution outside China: Korea.

Conium maculatum L., 1753, Sp. pl.: 243.

$\equiv$ Coriandrum cicuta Crantz, 1767 Stirp. Austr. 3: 100, nom. illeg. (Art. 52.1).

$\equiv$ Cicuta officinalis Crantz, 1767,Class. Umbell. Emend.: 98, nom. illeg. (Art. 52.1).

三 Cicuta major Lam., 1779, Fl. franç. 3: 456, nom. illeg. (Art. 52.1).

三 Coriandrum maculatum (L.) Roth, 1788, Tent. Fl. Germ. 1: 130.

三Sium conium Vest, 1806, Man. Bot.: 513, nom. illeg. (Art. 52.1).

三 Selinum conium E. H. L. Krause, 1904, in Sturm (ed.), Deutschl. Fl. (ed. 2), 12: 79, nom. illeg. (Art. 52.1).

Type: EUROPE. "In Europae cultis, agris, ruderatis, 343.1" (lectotype - LINN, designated by Jafri in Jafri, El-Gadi, 1985: 60) (see also: J.-P. Reduron, 2007: 877).

= Conium maculatum L. var. leiocarpum Boiss., 1872, Fl. Orient. 2: 922.

= Conium leiocarpum (Boiss.) Stapf et Wettst., 1886, Denkschr. Akad. Wien 51: 323.

Type: TURKEY. "In Tauro Cilicico. Intérieur du village du Gülek-Boghas, près du defilé du Portus Cilicicus, 06. 07. 1855, Balansa 563, 607" (syntypes G-Bois.!, JE!, barcode JE00000108, MPU, barcode MPU020975, P, barcodes P03378136, P03378169, WAG). IRAN. "Prov. Khorassan Pers.[Chorassan, inter Schahrud et Nischapur], 06. 1858, Bunge" (syntypes G-Bois.!, LE!, P!, barcodes P03378103, P03378155); "Persia austro-occidentalis, ad rivulos inter dumetis Teng Nalli. Monte Kuh Gelar, 7000', 07. 1868, Haussknecht" (lectotype G-BOIS!, barcode G00150080, designated by Pimenov, Jacquemoud in Pimenov, Ostroumova, 2012: 117; isolectotype JE!, barcode JE00000109).

Distribution in China: NW: Xinjiang Weiwuer A. R.

Distribution outside China: Russia, Vietnam, India, Pakistan, Kazakhstan, Kirghizia, Tajikistan, Turkmenistan, Afghanistan, Iran, Azerbaijan, Georgia, Armenia, Turkey, Iraq, Saudi Arabia, Yemen, Lebanon, Syria, Israel, Jordan, Cyprus, Greece (E Aegean Ils). Europe, N. Africa, N. America (adv.), S. America (adv.), S. Africa (adv.), Australia and Oceania (adv.).
Coriandrum sativum L., 1753, Sp. pl.: 256.

$\equiv$ Coriandrum globosum Salisb., 1796, Prodr. Stirp. Chap. Allerton: 166, nom. illeg. (Art. 52.1).

三Sium coriandrum Vest, 1806, Man. Bot.: 513, nom. illeg. (Art. 52.1).

三Selinum coriandrum E. H. L. Krause, 1904, in Sturm (ed.), Deutschl. Fl. (ed. 2), 12: 163.

Type: EUROPE. "In hortis Musniae, Daniae \& c., VIII 38" (lectotype UPS-Burser, designated by Jansen, 1981: 60).

Distribution in China: Tibet: Xizang A. R., Qinghai; N: Ningxia Huizu A.R., Shanxi, Shandong; NE: Heilongjiang, Liaoning; Centr.: Henan; SW: Sichuan, Guizhou, Yunnan; S: Guangxi Zhuang A. R., Guangdong, Hainan Dao.

Distribution outside China: Russia, Japan, Korea, Indonesia, Vietnam, Laos, Myanmar, Bangladesh, Bhutan, Nepal, India, Sri-Lanka, Pakistan, Kazakhstan, Kirghizia, Tajikistan, Uzbekistan, Turkmenistan, Afghanistan, Iran, Azerbaijan, Georgia, Armenia, Turkey, Iraq, Oman, Saudi Arabia, Yemen, Lebanon, Syria, Israel, Jordan, Egypt (Sinai), Cyprus, Europe, Africa, N. America, S. America, Australia and Oceania. Cultivated and adventive.

Cortia depressa (D. Don) C. Norman, 1937, J. Bot. 75: 96.

$\equiv$ Athamanta depressa D. Don, 1825, Prodr. Fl. Nepal.: 184.

$\equiv$ Cortia lindleyi DC., 1830, Prodr. 4: 187, nom. illeg. (Art. 52.1)

Type: NEPAL. "In Emodo Nepaliae ad GossainThan et Kamaon [In regione alpina Gosain Than, in jugo Emodi], Wallich [589] p.p." (lectotype K-WALLICH!, barcode K000685289, designated here; isolectotypes BM!, barcode BM000622297, CAL, E!, barcode E00002387, G-DC!, barcode G00367496, K!, barcode K000685391, LE!).

$=$ Cortia nepalensis C. Norman, 1929, J. Bot. 67: 245.

= Schulzia nepalensis (C. Norman) M. Hiroe, 1979, Umbelliferae World: 765.

Type: NEPAL. "Goshai Kand, 17000 ft., Wigram 63" (holotype E!, barcode E00018037).

$=$ Pachypleurum nyalamense Chang Hotseng et Shan Renhwa ex Shan Renhwa et al., 1980, Acta Phytotax. Sin. 18(3): 376.

Type: CHINA. "Xizang (Tibet): Nyalam Xian, alt. 35-3600 m, 31. 08. 1972, Tibetian Medicinal Herb. Exped. 1692" (holotype HMWP; isotype KUN!). 
Distribution in China: Tibet: Xizang A. R.; SW: Sichuan, Yunnan.

Distribution outside China: Bhutan, Nepal, India, Pakistan.

Cortia Ihasana (Chang Hotseng et Shan Renhwa) Pimenov et Kljuykov, 2005, Feddes Repert. 116 (12): 89, fig. 15.

$\equiv$ Pachypleurum lhasanum Chang Hotseng et Shan Renhwa, 1980,Acta Phytotax. Sin. 18(3): 377.

Type: CHINA. "Xizang: Lhassa, alt. 4600 m, 31. 08. 1965, Y. T. Chang et K. Y. Lang 2270" (holotype $\mathrm{PE}$; isotype $\mathrm{KUN}$ !).

Distribution in China: Tibet: Xizang A. R.; SW: Sichuan. Endemic.

Cortiella caespitosa Shan Renhwa et Sheh Menglan, 1980,Acta Phytotax. Sin. 18(3): 376.

Type: CHINA. "Xizang (Tibet): Nyemo Xian, alt. 5200 m, 09. 10. 1972, Xizang Exped. C 1692" (holotype PE).

Distribution in China: Tibet: Xizang A. R., Qinghai. Endemic.

Cortiella cortioides (C. Norman) M. F. Watson, 1996, Edinburgh J. Bot. 53(1): 130.

$\equiv$ Selinum cortioides C. Norman, 1937, J. Bot. 75: 95 .

Type: INDIA. "Sikkim, Phalloot, 13000 ft., 10.1877, Kurz" (lectotype K!, barcode K000685385, designated here; photo E); "Yakla, alt. $16000 \mathrm{ft}$., 18.10.1861, Clarke 9907, 9911" (syntype K!, barcodes K000685383, K000685383).

Distribution in China: Tibet: Xizang A.R.

Distribution outside China: Bhutan, Nepal, India.

Cortiella hookeri (C. B. Clarke) C. Norman, 1937, J. Bot. 75: 94, fig. B.

$\equiv$ Cortia hookeri C. B. Clarke, 1879, in Hooker f. (ed.), Fl. Brit. India 2: 702.

$\equiv$ Schulzia hookeri (C. B. Clarke) M. Hiroe, 1879, Umbelliferae World: 763, fig. 173.

Type: INDIA. "Sikkim, reg. alp., alt. 13000$18000 \mathrm{ft}$, Jongri, Hooker" (lectotype K!, designated by C. Norman, 1937: 94); isolectotypes E!, barcode E00002365, G, barcodes G00367494, G00397501, GH, LE!, M, barcode M0172875, MH!, MPU, barcode MPU020979).

$=$ Cortiella glacialis Bonner, 1879, Candollea 13: 230 .

= Pleurospermum glacialis (Bonner) M. Hiroe, 1979, Umbelliferae World: 747.
Type: NEPAL. "Rive droit du glacier du Kangchendzonga, 1949, Wyss-Dunant 1174" (lectotype G!, barcode G00367500, designated here; isolectotype BM, barcode BM000521558).

$=$ Cortiella cauwetmarciana Farille et S. B. Malla, 1985, Candollea 40(2): 543, fig. 11.

Type: BHUTAN. "Kerigla, 15000 feet, 01. 08. 1915, Cooper 4268" (holotype E).

Distribution in China: Tibet: Xizang A. R.

Distribution outside China: Bhutan, Nepal, India.

Cryptotaenia japonica Hassk., 1856, Retzia 1: 113.

$\equiv$ Cryptotaenia canadensis (L.) DC. var. japoni$c a$ (Hassk.) Makino. 1907, in Iinuma (ed.), SomokuDzusetsu (3 ed.) 1: 379, tab. 29.

$\equiv$ Deringa japonica (Hassk.) Koso-Pol., 1916 [1915], Monit. Jard. Bot. Tiflis 11: 139.

$\equiv$ Cryptotaenia canadensis (L.) DC. subsp. japonica (Hassk.) Hand.-Mazz., 1933, Symb. Sin. 7(3): 713.

Type: JAPAN. "Habit. Japoniam".

= Sanicula japonica Sieb., 1830, Verh. Batav. Gen. 12: 46, nom. inval., sine descr. (Art. 32.1)

- Cryptotaenia canadensis auct. non DC.: Sieb. \& Zucc., 1845, Abh. Akad. Muench. 4(3) (Pl. Jap. Fam. Nat. 2): 203.

Distribution in China: NW: Gansu; N: Shaanxi, Shanxi, Hebei; NE: Liaoning; Centr.: Henan, Jiangsu, Anhui, Hubei, Hunan, Jiangxi, Zhejiang, Fujiang; SW: Sichuan, Guizhou, Yunnan; S: Taiwan, Guangxi Zhuang A.R., Guangdong.

Distribution outside China: Russia, Japan, Korea, Indonesia, Vietnam.

Cryptotaenia japonica Hassk. f. pinnatisecta Liou Shoulu, 1990, Acta Phytotax. Sin. 28(2): 152.

Type: CHINA. "Guangxi: Longsheng, 27. 06. 1962, L. F. Liou et S. F. Yuan 5430" (holotype NAS).

Distribution in China: S: Guangxi Zhuang A.R.

Cuminum cyminum L., 1753, Sp. pl.: 254.

ELigusticum cuminum Crantz, 1767, Class. Umbell. Emend.: 82.

$\equiv$ Cuminum odorum Salisb., 1796, Prodr. Stirp. Chap. Allerton: 165, nom. illeg. (Art. 52.1).

三Luerssenia cyminum (L.) Kuntze, 1891, Revis. Gen. P1. 1: 268.

ESelinum cuminum E. H. L. Krause, 1904, in Sturm (ed.), Deutschl. Fl. (ed. 2) 12: 91.

Type: AFRICA. "In Aegypto, Aethiopia, 358.1" (lectotype LINN, barcode LINN-HL358-1, designated by Jansen, 1981: 71). 
- Cuminum hispanicum auct. non Merat: Bunge, 1851, Beitr. F1. Russl. (A1. Lehmann Reliq. Bot.): 312 .

Distribution in China: NW: Xinjiang Weiwuer A. R.

Distribution outside China: Myanmar, Bhutan, India, Pakistan, Kazakhstan, Uzbekistan, Turkmenistan, Afghanistan, Iran, Azerbaijan, Armenia, Iraq, Saudi Arabia, Europe, Africa.

Cyclorhiza peucedanifolia (Franch.) Constance, 1997, Edinburgh J. Bot. 54(1): 101, fig. 1.

三Arracacia peucedanifolia Franch., 1894, Bull. Annuel Soc. Philom. Paris (sér. 8) 6: 114.

Type: CHINA. "Yunnan: in fissuris in calcareous rock, Mount Pee-cha-ho, près de Mo-so-yn, 31. 08. 1884, Delavay 946" (lectotype P!, barcode P00057005, designated by Constance, 1997: 103); "Yunnan, Hoang-li-pin, supra Tapin-tze, in pratis, 16. 08. 1888, Delavay 4586" (syntype P); "in collibus calcareis supra Che-tong, alt. $1500 \mathrm{~m}$, Delavay 2026" (syntype P).

= Cenolophium chinense M. Hiroe, 1958, Umbell. Asia 1: 141.

Type: CHINA. "Prov. Yunnan: open dry situations in thickets on the Yung-pe mountains, Lat. $26^{\circ} 45^{\prime}$ N, Alt. 9000 feet, 04.1913, Forrest 11081" (holotype ("type") UC!, barcode UC231208; isotypes BM!, E!, barcode E00000122).

$=$ Cyclorhiza waltonii (H. Wolff) Sheh Menglan et Shan Renhwa var. major Sheh Menglan et Shan Renhwa, 1980, Acta Phytotax. Sin. 18(1): 46.

$=$ Cyclorhiza major (Sheh Menglan et Shan Renhwa) Sheh Menglan (eds.), in Shan Renhwa et Sheh Menglan, 1992, Fl. Reipubl. Popularis Sin. 55(3): 236, tab. 105, fig. 1-6.

Type: CHINA. "Yunnan: Dengchuan, 2800 m., Ching 24750" (holotype PE; isotypes KUN!, NAS!).

- Cyclorhiza edosmioides Sheh Menglan, 1997, in Wu Zhengyi (ed.), Fl. Yunnan. 7: 451, tab. 131, fig. 1-6, non Pimpinella edosmioides H. Boissieu.

Distribution in China: Tibet: Xizang A. R.; SW: Sichuan, Yunnan. Endemic.

Cyclorhiza waltonii (H. Wolff) Sheh Menglan et Shan Renhwa, 1980,Acta Phytotax. Sin. 18(1): 46, fig. 1.

三Ligusticum waltonii H. Wolff, 1930, Repert. Spec. Nov. Regni Veg. 27: 317.

ESeseli waltonii C. B. Clarke ex H. Wolff, 1930, Repert. Spec. Nov. Regni Veg 27: 317, nom. inval. (Art. 36.1), in syn.
Type: CHINA. "Tibet: Gyangtse, 07-09.1904, Walton 83" (lectotype K! - barcode K000685235, designated as “type' in $\mathrm{K}$ by $\mathrm{Pu}$ Fating and here by Pimenov; isolectotype $\mathrm{K}$ - barcode K000685234); "'[Everest Expedition] Tinki, stony ground, 13500', 14. 07. 1922, Norton 365" (syntype K! - barcode K000685236).

= Peucedanum Ihasense C. B. Clarke ex H. Wolff, 1933, Repert. Spec. Nov. Regni Veg. 33: 249.

Type: CHINA. "Tibet: hills above Lhasa, 08. 1904, Walton" (holotype K! - barcode K000685237).

Distribution in China: Tibet: Xizang A. R.; SW: Sichuan, Yunnan. Endemic.

Cyclospermum leptophyllum (Pers.) Sprague ex Britt. et P. Wilson, 1923, J. Bot. 61: 131.

EPimpinella leptophylla Pers., 1805, Syn. P1. 1: 324.

三Aethusa leptophylla (Pers.) Spreng., 1812, Mag. Neuesten. Entdesk. Gesammten Naturk. Ges. Naturf. Freude Berlin 6: 260.

ESison capillaceum Spreng.,1824, Syst. Veg. (ed. 16) 1: 887, nom. illeg. (Art. 52.1).

$\equiv$ Helosciadium leptophyllum (Pers.) DC., 1828, Mém. Soc. Phys. Genève 4: 493.

三Apium leptophyllum (Pers.) F. Muell., 1864, Fragm. Phytogr. Ausral. 4: 184.

三Selinum leptophyllum (Pers.) E. H. L. Krause, 1904, in Sturm (ed.), Deutschl. Fl. (ed. 2) 12: 38.

EPtychotis leptophylla (Pers.) Penz., 1905, Contrib. Storia Bot.: 21.

Type: AMERICA. "Ins. St.Dominica", Poiteau (syntype P); Bolivia, 1883-1889 M. Bang 1805" (syntype MO - barcode MO-345283).

= Cyclospermum ammi Lag., 1821, Amen. nat. Españ. 2: 101, "Ciclospermum".

= Apium ammi (Lag.) Urb., 1879, in Martius (ed.), Fl. Brasil. 11(1): 341, nom. illeg. (Art. 53.1), non Crantz (1767).

Type: "In Europa et America".

=Cnidium tenuifolium Moench, 1794, Methodus: 98.

= Apium tenuifolium (Moench) Thell., 1926, in Hegi (ed.), Ill. Fl. Mitt.-Eur. 5(2): 1140, in obs.

- Sison ammi auct. non L.: Jacq., Hort. bot. Vindob. 2: 95. 1772.

Distribution in China: Centr.: Jiangsu, Anhui, Hubei, Fujiang; S: Taiwan, Guangxi Zhuang A.R., Guangdong.

Distribution outside China: Russia, Japan, Indonesia, Malaysia, Myanmar, India, Sri-Lanka, Pakistan, Iran, Georgia, Israel. Europe, Africa, N. America, Centr. America, S. America, Australia and Oceania. 
Daucus carota L., 1753, Sp. pl.: 242.

$\equiv$ Caucalis daucus Crantz, 1767, Stirp. Austr., fasc. 3: 125, nom. illeg. (Art. 52.1).

$\equiv$ Caucalis carota (L.) Crantz, 1767, Class. Umbell. Emend.: 113.

$\equiv$ Daucus vulgaris Lam., 1779, Fl. Franç. 3: 430, nom. illeg. (Art. 52.1).

Type: EUROPE. "In Europae campis exaridis, 340.1" (lectotype LINN! - barcode LINN-HL340-1, designated by Sainz Lain, 1981: 487); cf. Wijnheimer et al. (1988).

= Daucus bactrianus Bunge, 1851, Beitr. Fl. Russl. (Al. Lehmann Reliq. Bot.): 136.

Type: UZBEKISTAN. "Am Sarafschan unweit Samarkand, 13. 10. 1841, Lehmann" (holotype P?).

= Daucus exarmatus Korovin, 1948, Not. Syst. Herb. Inst. Bot. \& Zool. Acad. Sci. Uzbekistan 12: 23.

Type: TAJIKISTAN. "Pamir-alaj, in valle fluv. Kafirnigan meridiem versus urb. Stalinabad [Duchambe], 12. 07. 1930, Pazij et Mironov 1342" (holotype TASH!).

- Daucus aureus auct. non Desf.: Tchih., 1860, As. Min., Bot. 1: 447.

Distribution in China: NW: Xinjiang Weiwuer A. R., Gansu; Tibet: Xizang A. R.; N: Neimenggu A. R., Ningxia Huizu A.R., Shaanxi, Shanxi, Hebei, Shandong; Centr.: Henan, Jiangsu, Anhui, Hubei, Hunan, Jiangxi, Zhejiang, Fujiang; SW: Sichuan, Guizhou, Yunnan; S: Guangxi Zhuang A. R., Guangdong.

Distribution outside China: Russia, Japan, Nepal, India, Sri-Lanka, Pakistan, Kazakhstan, Kirghizia, Tajikistan, Uzbekistan, Turkmenistan, Afghanistan, Iran, Azerbaijan, Georgia, Armenia, Turkey, Iraq, Oman, UAE, Saudi Arabia, Yemen, Lebanon, Syria, Israel, Jordan, Cyprus, Greece (E Aegean Ils). Europe, Africa, N. America, Australia and Oceania.

Dickinsia hydrocotyloides Franch., 1888, Nuov. Arch. Mus. Hist. Nat. (sér. 2) 8 (Pl. David. 2): 244, tab. 8, fig. A.

Type: CHINA. "Thibet oriental, Moupine, in silvis humidis, 08. 1859, David" (holotype P! barcode P00835133).

= Cotylonia bracteata C. Norman, 1922, J. Bot. 60: 167, fig. A-D.

Type: CHINA. "Western China: roadside. Wilson 3666" (lectotype BM! - barcode BM000944683, designated here; isolectotype K); "China S.Wushan. Wilson 2011" (syntypes A - barcode A00062636,
K); “China, Mt. Orni, Wilson 4930" (syntype A barcode A00062637).

Distribution in China: Centr.: Hubei, Hunan; SW: Sichuan, Guizhou, Yunnan. Endemic.

Dimorphosciadium gayoides (Regel et Schmalh.) Pimenov, 1975, Byull. Moskovsk. Obšč. Isp. Prir. Otd. Biol. 80(3): 83.

$\equiv$ Meum gayoides Regel et Schmalh., 1881, Izv. Obšč. Ljubit. Estestv. 34(2) (Descr. Pl. Nov. Rar. Fedtsch.): 32.

$\equiv$ Ligusticum gayoides (Regel et Schmalh.) Korovin, 1925, Bull. Inst. Pedol. \& Geobot. Univ. As. Centr. 1: 108, in nota.

$\equiv$ Pachypleurum gayoides (Regel et Schmalh.) Schischk., 1950, in Schischkin (ed.), Fl. URSS 16: 582, p.p.

Type: KIRGHIZIA. "Kokand, traj. KitschikAlaj, 26. 07. 1871, O. A. Fedtschenko" (holotype LE!).

Distribution in China: NW: Xinjiang Weiwuer A. R.

Distribution outside China: Kazakhstan, Kirghizia, Tajikistan, Uzbekistan, Afghanistan.

Dimorphosciadium shenii Pimenov et Kljuykov, 2001, Acta Phytotax. Sin. 39(3): 197.

$\equiv$ Chamaesciadium acaule (M. Bieb.) Boiss. var. simplex Shan Renhwa et Pu Fating, 1983, Acta Phytotax. Sin. 21(1): 81.

Type: CHINA. "Kashi. Kashi School of Health 017" (holotype XJDCMI!).

Distribution in China: NW: Xinjiang Weiwuer A. R. Endemic.

Elwendia persica (Boiss.) Pimenov et Kljuykov, 2013, Pl. Syst. Evol. 299 (5): 1004.

$\equiv$ Carum persicum Boiss., 1844, Ann. Sci. Nat. Paris (sér. 3, Bot.) 1 (Pl. Aucher.): 138, nom. cons. prop.

三 Bunium persicum (Boiss.) B. Fedtsch., 1915, Rastit. Turkest.: 612.

Type: IRAN. "Persia australis, AucherEloy 4555" (lectotype G-BOIS! - barcode G00359481, designated by Geldykhanov, 1992: 122; isolectotypes $\mathrm{K}$ ! - barcode K000685695, P! barcodes P00834751, P00834752, US - barcode US00588543); "Inter Fasa et Chyraz, Aucher-Eloy 4553" (syntypes G! - barcode G00359482, P! barcode P00834750).

= Sium cyminosma Basiner, in Fisch.\& C. A. Mey. (eds.), 1843, Index Sem. Horti Petrop. 9, Suppl.: 18, nom. rej. prop. 
= Pimpinella cyminosma (Basiner) Koso-Pol., 1916, Bull. Soc. Imp. Naturalistes Moscou, s.n. 29: 180.

Type: UZBEKISTAN. "Seeds: Col. Chivensibus sub nomina ssara[e]-sir, 1843, Basiner" (lectotype LE!, designated here; isolectotype P!).

= Carum heterophyllum Regel et Schmalh., 1878, Trudy Imp. S.-Peterburgsk. Bot. Sada 5, 2: 586.

Type: TAJIKISTAN. "Zeravschan, inter Varsaminor et Peti, 4500-6000', 12. 06. 1870, O. A. Fedtschenko" (lectotype LE!, designated by Vinogradova, 1997a: 98).

Distribution in China: NW: Xinjiang Weiwuer A. R.

Distribution outside China: Pakistan, Kirghizia, Tajikistan, Uzbekistan, Turkmenistan, Afghanistan, Iran.

Elwendia setacea (Schrenk) Pimenov et Kljuykov, 2013, Pl. Syst. Evol. 299 (5): 1004.

$\equiv$ Carum setaceum Schrenk, 1841, Enum. P1.

Nov. 1: 61.

$\equiv$ Conopodium setaceum (Schrenk) Korovin, 1924, Bull. Sredne-Asiatsk. Gosud. Univ. 7, Suppl. (Sched. Herb. Fl. As. Med. 1-2): 24.

三Bunium setaceum (Schrenk) H. Wolff, 1927, in Engler (ed.), Pflanzenreich 90(IV, 228): 209.

EScaligeria setacea (Schrenk) Korovin, 1928, Bull. Sredne-Asiatsk. Univ. (ser. 8b, Bot.) 2: 67.

Type: KAZAKHSTAN. "In fruticetis collium Songoriae ad rivulum Ai, hinc inde, in montibus Alatau ad fl. Lepsa et Baskan [Songarei, Arganaty], 01.06.1840, Schrenk" (lectotype LE!, designated by V. M. Vinogradova, 1997a: 98).

= Carum capillifolium Kar. et Kir., 1841, Bull.

Soc. Imp. Naturalistes Moscou 14: 428.

= Bunium capillifolium auct. non Bertol.: Botsch., 1976, Novosti Sist. Vyssh. Rast. 13: 249.

Type: KAZAKHSTAN. "In fruticetis collium Soongoriae ad rivulum $\mathrm{Ai}$, hinc inde, in montosis Alatau ad fl. Lepsa et Baksan, 1841, Karelin et Kirilov 1531" (neotype MW!, designated by Kljuykov et al., 1977: 143; isoneotypes LE!, M!).

= Carum setaceum Schrenk var. borgatense Lipsky, 1904, Trudy Imp. S.-Peterburgsk. Bot. Sada 23: 126

Type: CHINA. "Kuldsha, fliv. Borgaty (Kasch). 08. 07. 1879, A. Regel" (holotype LE!).

Distribution in China: NW: Xinjiang Weiwuer A. R.

Distribution outside China: Mongolia, Kazakhstan, Kirghizia, Uzbekistan, Turkmenistan.
Eryngium foetidum L., 1753, Sp. pl.: 232.

Type: ICON JAMAICA. "Eryngium foliis angustis senatis foetidum in Sloane, Fl. Jamaica: tab. 156, fig. 3 (1707)" (lectotype, designated by Wörz, 1999: 19).

= Eryngium antihystericum Rottl., 1778, Acta Lit. Univ. Hafn. 1: 288.

Distribution in China: SW: Guizhou, Yunnan; S: Taiwan, Guangxi Zhuang A.R., Guangdong, Hainan Dao.

Distribution outside China: Philippines, Indonesia, Malaysia, Vietnam, Laos, Cambodia (cult.), Thailand, Myanmar, Bangladesh, Bhutan, Nepal, India, Sri-Lanka, Africa, N. America, Centr. America, S. America.

Eryngium macrocalyx Schrenk, 1841, Enum. P1. Nov. 1: 60.

Type: KAZAKHSTAN. "In desertis Songariae, ad rivulum Kinasch [Songaria, in deserti ad fl. Kunasch], 17. 07. 1840, Schrenk" (lectotype LE!; designated here; isolectotype MANCH!).

= Eryngium incognitum Pavlov, 1938, Byull. Moskovsk. Obšč. Isp. Prir. n.s. 47(1): 81.

Type: TAJIKISTAN. "Tadshikistania, in collibus prope Stalinabad [Dushanbe], $1000 \mathrm{~m}$ alt., 08. 07. 1932, Stschukin" (holotype MW!).

= Eryngium pamiroalaicum Korovin, 1947, Not. Syst. Herb. Inst. Bot. \& Zool. Acad. Sci. Uzbekistan 8: 3 .

Type: TAJIKISTAN. "Pamir-Alaj meridionalis, montes Baba-tag prope p. Dshida-bulak, 03. 07. 1936, Lepeschkin et Muchamedshanov 389" (holotype TASH!).

- Eryngium polycephalum auct. non Hausskn. ex H. Wolff: Rech. f. \& Riedl, 1963, K. Danske Vid. Selsk., Biol. Skrift. 13, 4 (Symb. Afghan. 5): 56.

Distribution in China: NW: Xinjiang Weiwuer A. R.

Distribution outside China: Kazakhstan, Kirghizia, Tajikistan, Uzbekistan, Afghanistan.

Eryngium planum L., 1953, Sp. pl.: 233.

Type: EUROPE. "[Danube near Vienna, and. Silesia]? Herb. Clifford, 87, Eryngium 1, Fol. A" (lectotype BM-Cliff - barcode BM000558217, designated by Jury and Southam in: Jarvis et al., 2006: 212).

Distribution in China: NW: Xinjiang Weiwuer A. R.

Distribution outside China: Russia, Mongolia, Kazakhstan, Georgia. Europe, N. America. 
Ferula akitschkensis B. Fedtsch. ex Koso-Pol., 1925, Byull. Obšč. Estestvoisp. Voronezsk. Gosud. Univ. 1: 94.

Type: KAZAKHSTAN. "Prov. Heptapotamica, jugum Dzhungarsky Alatau, prope stat. Ak-Itschke, steppa, 20. 07. 1908, B. A. Fedtschenko 1633" (holotype LE!; isotype LE!).

$=$ Ferula transitoria Korovin, 1947, Gen. Ferula Monogr. Ill.: 70, p.p.

Type: KAZAKHSTAN. "Mont. Dshungarienses, Tschartombaj, 16. 06. 1909, Roshevitz 81" (lectotype LE!, designated here).

$=$ Ferula angustiloba Pimenov, 1976, Bot. Zhurn. (Leningrad) 61(9): 1230.

Type: KIRGHIZIA. "Tian-Schan septentrionalis, Kirghizia, jugum Kungei-Alatau, declivitas australis prope trajectum Santach, ad marginem piceeti, 17. 07. 1970, Pimenov et Trusov 751" (holotype LE!; isotype MW!).

= Ferula akitschkensis B. Fedtsch. ex KosoPol. var. kuldshensis Korovin, 1947, Gen. Ferula Monogr. Ill.: 70.

Type: CHINA. "Kuldsha".

- Ferula karataviensis auct. non Korovin: Shen Kuanmien, 1992, in Shan Renhwa \& Sheh Menglan (eds.), Fl. Reipubl. Popularis Sin. 55(3): 114.

- Ferula kirialovii auct. non Pimenov: Shen Kuanmien, 1992, in Shan Renhwa \& Sheh Menglan (eds.), Fl. Reipubl. Popularis Sin. 55, 3: 106, tab. 45, fig. 1-3.

- Ferula sumbul auct. non Hook. f.: Shen Kuanmien, 1992, in Shan Renhwa \& Sheh Menglan (eds.), Fl. Reipubl. Popularis Sin. 55, 3: 105.

Distribution in China: NW: Xinjiang Weiwuer A. R.

Distribution outside China: Kazakhstan, Kirghizia.

Ferula bungeana Kitag., 1956, J. Jap. Bot. 31: 304.

$\equiv$ Peucedanum rigidum Bunge, 1835, Enum. Pl. Chin. Bor: 32. 1833; Mém. Acad. Imp. Sci. St. Petersburg Divers Savans 2: 106.

Type: CHINA. "China borealis, 1831, Bunge 662" (lectotype P! - barcode P02272136, designated here; isolectotypes G! - barcode G00366819, K!, L!. LE!, M - barcode M0173070, P, PE!).

- Ferula rigidula auct. non DC.: M. Hiroe, 1958. Umbell. Asia 1: 185.

Distribution in China: NW: Gansu; Tibet: Qinghai; N: Neimenggu A. R., Ningxia Huizu A.R., Shaanxi, Shanxi, Hebei; NE: Heilongjiang, Jilin, Liaoning; Centr.: Henan.
Distribution outside China: Mongolia.

Ferula canescens (Ledeb.) Ledeb., Fl. Ross. 2: 302. 1844.

$\equiv$ Peucedanum canescens Ledeb., Fl. Altaic. 1: 307. 1829.

Type: KAZAKHSTAN. "In rupestribus montis Arkaul, Meyer 236" (lectotype LE!, designated by Vinogradova in LE and validated here; isolectotype $\mathrm{P} !)$.

Distribution in China: NW: Xinjiang Weiwuer A. R.

Distribution outside China: Kazakhstan, Uzbekistan.

Ferula caspica M. Bieb., 1808, Fl. Taur.-Caucas. 1: 220 .

三Peucedanum caspicum (M. Bieb.) Link, 1821, Enum. Hort. Berol. Alt. 1: 272.

Type: RUSSIA. "In deserto caucasico-caspica, ad viam publicam inter Astrachan et Kisljar [Ex planitiebus oppida Kisljar vicinis], Marchall von Bieberstein" (lectotype LE-Bieb!, designated by Menitski, 2008: 99; isolectotype MW-Trinius).

$=$ Ferula pumila Pall. ex Spreng., 1820, in Roemer \& Schultes (eds.), Syst. Veg. (ed. 15 bis) 6: 598 .

Type: RUSSIA. "Ad Wolgam, Pallas" (lectotype B-Willd!, designated here; isolectotype HAL barcode HAL0033090).

= Peucedanum gracile Ledeb., 1829, Fl. Altaic. 1: 308 .

$=$ Ferula gracilis (Ledeb.) Ledeb., 1844, Fl. Ross. 2: 304.

Type: KAZAKHSTAN. "[Altai], in apricis, siccis, subsalsis deserti soongoro-kirghisici inter Buchtarminsk et lacum, qui Noor-Saisan vocatur, 1826, Meyer 238" (lectotype LE!, designated by Vinogradova, 2001: 54; isolectotypes HAL, P barcode P02272152, PH - barcode PH00023034).

= Peucedanum gracile Ledeb. var. microcarpum Ledeb., 1829, Fl. Altaic. 1: 309.

Type: KAZAKHSTAN. "Altai, in lapidosis ad fl. Grammatucha prope Riddersk, Ledebour" (lectotype LE?; isolectotype CGE!)

= Peucedanum dubium Ledeb., 1829, Fl. Altaic. 1: 310 .

Type: RUSSIA. "Altai, in apricis siccis sterilissimis deserti editi ad fl. Tschuja mediis, 1826, Bunge [ 237]" (lectotype LE!, designated here; isolectotypes C - barcode C10008434, CGE!, LE!, $\mathrm{P}$ - barcodes P02272153, P02272154, P02272155).

= Ferula wolgensis Willd. ex Ledeb., 1844, Fl. Ross. 2: 203, p.p. 
Type: RUSSIA. "Ad Wolgam" (syntypes B-Willd. - barcodes B-W 05773 01-04, 06) cf. Ferula pumila.

Distribution in China: NW: Xinjiang Weiwuer A. R.

Distribution outside China: Russia, Mongolia, Kazakhstan.

Ferula dissecta (Ledeb.) Ledeb., 1844, Fl. Ross. 2: 301 .

$\equiv$ Peucedanum dissectum Ledeb., 1829, F1. Altaic. 1: 306.

Type: KAZAKHSTAN. "In collibus ad rivulum Talowka prope Buchtarminsk, 17. 05. 1826, Meyer 239" (lectotype LE!, designated here; isolectotypes P! - barcodes P02272139, P02272140).

= Peucedanum ledebourii Steud., 1841, Nomencl. Bot. ed. 2 2: 311, nom. illeg., non G. Don (1834).

$=$ Ferula rigidula DC. var. songorica Schrenk ex Fisch. \& C. A. Mey., 1842, in Schrenk, Enum. P1. Nov. 2: 43.

Type: KAZAKHSTAN. "In collibus prope Ajagus, Schrenk" (lectotype LE!, designated here; isolectotypes W!, WU-Keck!).

Distribution in China: NW: Xinjiang Weiwuer A. R.

Distribution outside China: Mongolia, Kazakhstan.

Ferula dshaudshamyr Korovin, 1947, Gen. Ferula Monogr. Ill.: 79, tab. 16, fig. 1.

$\equiv$ Dorema songaricum Kar. et Kir., 1842, Bull. Soc. Imp. Naturalistes Moscou 15: 365, non Ferula soongarica Pall. ex Spreng.

三 Ferula dubjanskyi Korovin ex Pavlov [1935, Bull. Sredne-Asiatsk. Gosud. Univ. 20: 196, nomen] 1935, Fl. Centr. Kazakh. 2: 534, 539, nom. illeg. (Art. 39.1).

Type: KAZAKHSTAN. "In collibus sabulosis Songoriae inter fontem Sassyk-pastau et montes Arganaty, 1841, Karelin et Kirilov 1542" (lectotype LE!, designated by Vinogradova, 1999: 86; isolectotype $\mathrm{P}$ ).

Distribution in China: NW: Xinjiang Weiwuer A. R.

Distribution outside China: Mongolia, Kazakhstan, Uzbekistan.

Ferula ferulioides (Steud.) Korovin, 1947, Gen. Ferula Monogr. Ill.: 77.

$\equiv$ Peucedanum paniculatum Ledeb., 1829, F1. Altaic. 1: 310, nom. illeg., non Loisel. (1828). $\equiv$ Peucedanum soongaricum $\mathrm{G}$. Don, 1834 , Gen. Hist. 3: 332, non Ferula soongarica Pall. ex Spreng.

$\equiv$ Peucedanum ferulioides Steud., 1841, Nomencl. bot. (ed. 2) 2: 311 .

$\equiv$ Peucedanum pyramidatum Kar. et Kir., 1842, Bull. Soc. Imp. Naturalistes Moscou 15: 366, nom. illeg. (Art. 52.1).

$\equiv$ Ferula paniculata (Ledeb.) Ledeb., 1844, Fl. Ross. 2: 305, nom. illeg.

Type: KAZAKHSTAN. "In subsalsis deserti soongoro-kirghisici inter fl. Irtysch et montes Dolen-kara nec non in regione occidentali, Meyer 235" (lectotype LE!, designated here; isolectotypes CGE!, P! - barcodes P02272141, P02272142, P02272143).

Distribution in China: NW: Xinjiang Weiwuer A. R.

Distribution outside China: Mongolia, Kazakhstan.

Ferula foetidissima Regel et Schmalh., 1878, Gartenflora 27: 195, tab. 944; 1878, Trudy Imp. S.Peterburgsk. Bot. Sada 5(2): 593.

Type: TAJIKISTAN. "In valle fluvii Sarawschon prope Obburdon et Pachud, 5000`alt., 06. 06. 1870, O. A. Fedtschenko" (lectotype LE!, designated by Pimenov, Baranova, 1979: 86).

- Ferula conocaula auct. non Korovin: Shen Kuanmien, 1975, Acta Phytotax. Sin. 13(3): 91.

Distribution in China: NW: Xinjiang Weiwuer A. R.

Distribution outside China: Kirghizia, Tajikistan, Uzbekistan.

Ferula fukanensis Shen Kuanmien, 1975, Acta Phytotax. Sin. 13(3): 90, fig. 2.

Type: CHINA. "Xinjiang, Fukang, alt. 1700 m, 23. 05. 1974, Inst. Biol. Ped. and Desert. Res. CAS 791" (holotype XJBI!; isotype NAS!).

Distribution in China: NW: Xinjiang Weiwuer A. R. Endemic.

Ferula groessingii Riedl \& C. Riedl-Dorn, 1987, Linzer Biol. Beitr. 19(2): 485, fig. 1, 2.

Type: CHINA. "Sina borealis, prov. Shantung: Taitsenggong prope Tsingtao, 12. 08. 1936, Licent 13413" (holotype W!).

Distribution in China: N: Shandong. Endemic.

Ferula hexiensis Shen Kuanmien, 1986, Acta Phytotax. Sin. 24(4): 314, fig. 10.

Type: CHINA. "Gansu, Sunan, 07. 08. 1967, Exped. Hexi 101" (holotype XJBI; isotype PE!). 
Distribution in China: NW: Gansu; Tibet: Qinghai. Endemic.

Ferula iliensis Krasn. ex Korovin, 1947, Gen. Ferula Monogr. Ill.: 34.

Type: KAZAKHSTAN. "Asia Media, deserta Balhaschensis, monte Boguty et Soguty inter stat. Taldykuduk et Kisil-Kija, Krasnov" (holotype E; isotype $\mathrm{W} !)$.

$=$ Ferula popovii Korovin, 1962, Trudy Inst. Bot. Akad. nauk Kazakhsk. SSR 13: 258.

Type: KAZAKHSTAN. "Dshungarsky Alatau (pars meridionalis), mons Ulkunkalkan, ad planitiem argillaceo-petraeum, 21. 06. 1959, Sinicin" (syntype AA!); "In regione montis Bolschie Boguty, 26. 06. 1958, Bykov et Godvinski" (syntype AA!). A. R.

Distribution in China: NW: Xinjiang Weiwuer

\section{Distribution outside China: Kazakhstan.}

Ferula jaeschkeana Vatke, 1876, Ind. Sem. Horti Berol. append. 2.

$\equiv$ Peucedanum jaeschkeanum (Vatke) Baill., 1884, Traite Bot. Med. Phan. 2: 1043.

Type: INDIA. "In angustiis Rotang dictis Himalayae, 14-15000 ft., 1867, Jaeschke" (holotype B).

$=$ Ferula jaeschkeana Vatke var. parkeriana O.E.Schulz, 1933, Notizbl. Bot. Gart. Berlin-Dahlem 11: 877.

Type: PAKISTAN. "Nordwest Indien: Kashmir, Sonamarg, alt. 9000-11000 ft., 26. 07. 1928, Stewart 9786" (syntype NY); "Hazara, Abbottabad, alt. 4500 m, 23 04. 1930, Stewart 10775" (syntype).

$=$ Ferula wolffi Korovin, 1947, Gen. Ferula Monogr. Ill.: 35.

Type: INDIA. "India: montes himalayenses occidentales, in prov. Chamba, Pendshab. Lahol in val. fl. Baga. [Chamba, 3000 ft.], 03. 1889, Lace[1906]" (holotype TASH; isotype E!).

$=$ Ferula gangensis Korovin, 1947,Gen. Ferula Monogr. Ill.: 34.

Type: INDIA. "NW Himalaya, Tihri Garhwal, near Jangla in Sangea (? Gangea) valley, 9-10000' [Near Jangla in Ganges valley], 07. 07. 1893, Duthie 1139” (holotype LE!).

Distribution in China: Tibet: Xizang A. R.

Distribution outside China: India, Pakistan, Afghanistan.

Ferula karelinii Bunge, 1851, Beitr. F1. Russl. (Al. Lehmann Reliq. Bot.): 130.

三 Schumannia karelinii (Bunge) Korovin, 1947, Gen. Ferula Monogr. Ill.: 81.
Type: UZBEKISTAN. "In der Lehmsalzsteppe Karakum, 28. 06. 1841, Lehmann 544" (lectotype LE!, designated by Vinogradova, 2002b: 139; isolectotypes K, P); KAZAKHSTAN. "In alten Flussbette des Jam-Darja, 03.05.1842, Lehmann" (syntype $\mathrm{P}$ - barcode $\mathrm{P} 02272182$ ).

= Schumannia turcomanica Kuntze, 1887,Trudy Imp. S.-Peterburgsk. Bot. Sada 10: 192.

Type: TURKMENISTAN. "Zwischen KisilArwat und dem Kaspi-See".

- Ferula peucedanifolia auct. non Willd.: Kar. \& Kir., 1842, Bull. Soc. Imp. Naturalistes Moscou 15: 165.

Distribution in China: NW: Xinjiang Weiwuer A. R.

Distribution outside China: Pakistan, Kazakhstan, Tajikistan, Uzbekistan, Turkmenistan, Afghanistan, Iran, East Europe (Kalmykia).

Ferula kingdon-wardii H. Wolff, 1930, Repert. Spec. Nov. Regni Veg. 27: 326.

$\equiv$ Peucedanum kingdon-wardii (H. Wolff) Korovin, 1947, Gen. Ferula Monogr. Ill.: 81.

Type: CHINA. "Ost-Tibet: Yung-ning, alt. 10500 ft., 20. 04. 1922, Kingdon-Ward 5060" (holotype $\mathrm{E}$ !).

Distribution in China: SW: Yunnan. Endemic.

Ferula krylovii Korovin, 1934, Sist. Zametki Mater. Gerb. Krylova Tomsk. Gosud. Univ. 2-3: 2.

Type: KAZAKHSTAN. "Prov. Heptapotamica, distr. Zaisan, prope pag. Buran, m. Aschutas, steppa deserta subsalsuginosa, 09. 07. 1928, Krylov et Sergievskaya" (lectotype TK!, designated by Polozhij and Balashova (1989: 26), as "type"; isolectotypes LE!, TK).

- Ferula teterrima auct. non Kar. et Kir.: Krylov, 1935, Fl. Zapadnoi Sibiri 8: 1995.

Distribution in China: NW: Xinjiang Weiwuer A. R.

Distribution outside China: Kazakhstan.

Ferula leiophylla Korovin, 1947, Gen. Ferula Monogr. Ill.: 44, tab. 20, fig. 2.

Type: KAZAKHSTAN. "Distr. Kapal, steppa arenosa secundum riv. Yanach, 09. 06. 1915, Titov 41" (lectotype TASH!, designated here).

Distribution in China: NW: Xinjiang Weiwuer A. R.

Distribution outside China: Kazakhstan, Kirghizia.

Ferula licentiana Hand.-Mazz., 1933, Oesterr. Bot. Z. 82: 252. 
Type: CHINA. "Schansi, Taihang-shan, Toukoutwei, 19. 06. 1915, Licent 1156" (holotype P! - barcode P02272168).

Distribution in China: N: Shaanxi, Shanxi, Hebei, Shandong; Centr.: Henan, Jiangsu, Anhui. Endemic.

Ferula licentiana Hand.-Mazz. var. tunshanica (Shan Renhwa) Shan Renhwa et Liu QiXing [1987, Bull. Nanjing Bot. Gard.: 37, nom. inval.] ex Shen Kuanmien, 1992, in Shan Renhwa \& Sheh Menglan (eds.), Fl. Reip. Pop. Sin. 55(3): 114.

$\equiv$ Ferula tunshanica Shan Renhwa, Fl. Jiangsu 2: 584, 935, tab. 1678. 1982.

Type: CHINA. "Jiangsu, Tongshan Hsien, Maocun, 22. 06. 1974, Fang Wenzhe et Ling Pingping 74020" (holotype NAS!).

Distribution in China: N: Shandong; Centr.: Jiangsu, Anhui, Hubei. Endemic.

Ferula olivacea (Diels) H. Wolff ex Hand.Mazz., 1933, Symb. Sin. 7(3): 727.

三 Peucedanum olivaceum Diels, 1912, Notes Roy. Bot. Gard. Edinburgh 5: 290.

Type: CHINA. "Dry situations in shady pine forests on the eastern flank of the Lichiang Range. Lat. $27^{\circ} 12^{\prime}$ N. Alt. 9-10000 ft., 05. 1906, Forrest 2250" (lectotype E! - barcode E00002347, designated here; isolectotype P! - barcode P00752979).

Distribution in China: SW: Sichuan, Yunnan. Endemic.

Ferula ovina (Boiss.) Boiss. [1845, in Hohenakker (ed.). P1. Pers. Austr. N 283, nom. inval.], 1872, Fl. Orient. 2: 986.

EPeucedanum ovinum Boiss., 1846, Diagn. P1. Orient. (ser. 1) 6: 61.

Type: IRAN. "Persia austr., in rupestribus prope ruinas u. Persepolitanas, 22. 04. 1842, Kotschy 283.409" (lectotype G-BOIS!, designated here by Pimenov and Jacquemoud; isolectotypes BM! - barcodes BM000885452, BM000944771, C! - barcodes C10008441, C10008442, FI! barcode FI004421, FI-W!, E!, GOET - barcode GOET011338, HAL - barcode HAL0036472, JE! barcode JE0006704, K! - barcode K000568180, L! - barcode L 2576875, LE!, LIV!, M - barcode M0173053, MO - barcodes MO-345443, MO345444, P! - barcodes P02272187, P02272188, P02272189, S! - barcode S-G-2698, TI!, US, W, WAG - barcode WAG0004950).

$=$ Peucedanum thomsonii C. B. Clarke, 1879, in Hooker f. (ed.), Fl. Brit. India 2: 711.
Type: INDIA. "Kashmir, alt. 5000-9000 ft., Kishtwar, 10. 05. 1848, Thomson" (lectotype K! barcode K001097258, designated here; isolectotypes CAL, G! - barcode G00366775, K! - barcodes K001097256, K001097257, K001097259, P!, W!); "Kashmir, Banahal, Thomson" (syntype K! barcodes K001097260, K001097261).

= Ferula pachycarpa Korovin ex Pavlov (ed.), 1935, Fl. Centr. Kazakh. 2: 532, 537. nom. inval. (Art. 39.1).

= Ferula microcarpa Korovin, 1947, Gen. Ferula Monogr. Ill.: 58, tab. 33, fig. 2.

Type: KAZAKHSTAN. "Alatau Dshungariensis, distr. Kapal, Baj-Gazy, 11. 06. 1917, Titov” (holotype TASH!).

= Ferula stylosa Korovin, 1947, Gen. Ferula Monogr. Ill.: 58, tab. 34, fig. 2.

Type: KAZAKHSTAN. "M. Tschu-ilienses, in loco Ajderke, Uzun-bulak, declivia calcarea. 18. 06. 1926, Titov 676" (holotype TASH!).

= Ferula lapidosa Korovin, 1947, Gen. Ferula Monogr. Ill.: 59, tab. 35, fig. 1.

Type: KIRGHIZIA. "Kirghyzstan, in angust. Buam, prope pontem medium, rubroarenosa gypsacea, 27. 07. 1926, Abolin 639" (holotype TASH!).

Distribution in China: NW: Xinjiang Weiwuer A. R.

Distribution outside China: India, Pakistan, Kazakhstan, Kirghizia, Tajikistan, Uzbekistan, Turkmenistan, Afghanistan, Iran, Saudi Arabia, Yemen, Israel, Jordan.

Ferula potaninii Korovin [ex Pavlov, 1935, Fl. Centr. Kazakh. 2: 532, 537, nom. illeg., descr. ross.] 1947, Gen. Ferula Monogr. Ill.: 56, tab. 30, fig. 2.

Type: KAZAKHSTAN. "Asia Media, montes Tarbagatai [District Semipalatinsk, praemontia jugi Tarbagatai], Potanin" (holotype TK!).

Distribution in China: NW: Xinjiang Weiwuer A. R.

Distribution outside China: Mongolia, Kazakhstan.

Ferula sibirica Willd., 1798, Sp. pl. (ed. 4) 1(2): 1411.

三Ferula peucedanifolia Willd. ex Spreng., 1818, Sp. Umbell.: 87; 1820, in Roemer \& Schultes (eds.), Syst. Veg. (ed. 15 bis) 6: 592, nom. illeg.

三Peucedanum cephalotes Boiss., 1844, Ann. Sci. Nat. Paris (sér. 3, Bot.) 1 (P1. Aucher.): 314.

三Peucedanum pallasii Cusson ex Ledeb., 1844, F1. Ross., 2: 272, nom. inval. (Art. 36.1), in syn. 
$\equiv$ Ferula pallasii [Cusson ex Ledeb.] Koso-Pol., 1925, Byull. Obšč. Estestvoisp. Voronezsk. Gosud. Univ. 1: 37., in obs., nom. inval (Art. 32.1?) et 1925, Byull. Obšč. Estestvoisp. Voronezsk. Gosud. Univ. 1: 92, in clavi,

$\equiv$ Soranthus peucedanifolius (Willd.) Woronow, 1931, Trudy Bot. Sada Akad. Nauk URSS 43(2) (Flora Yugo-Vost. 5): 823.

$\equiv$ Soranthus sibiricus (Willd.) Korovin, 1947, Gen. Ferula Monogr. Ill.: 81.

Type: EUROPE, RUSSIA. "In Sibiria locis arenosis humidiusculis inter Wolgam et Jaikam, Pallas" (lectotype B-Willd! - barcodes B-W-0577201 0, B-W-05772-02 0, B-W-05772-03 0, designated here; isolectotype HAL - barcode HAL0036473).

= Soranthus meyeri Ledeb., 1829 (Maj-Dec.), Icon. Pl. 1: 20, tab. 82.; ejusd., 1829 (Nov.-Dec.). Fl. Altaic. 1: 345 .

$=$ Seseli meyeri (Ledeb.) D.Dietr., 1840, Syn. P1. 2: 956.

= Ferula meyeri (Ledeb.) Bunge, 1851, Beitr. Kenntn. Fl. Russl. (Al. Lehmann Reliq. Bot.): 131; 1851, Mém. Sav. Etrang. Petersb. 7: 307.

Type: KAZAKHSTAN. "In arenosis ad fl. Irtysch non procul a lacu, qui Noor-Saisan vocatur et ad fl. Bekun, Meyer 265" (lectotype LE!, designated here; isolectotypes MO - barcode MO-2196579, P! barcodes P02272175, P02272176).

$=$ Ferula capillifolia Stschegl., 1854, Bull. Soc. Imp. Naturalistes Moscou 27(1): 168.

Type: KAZAKHSTAN. "In sabulosis ad fl. Irtysch, 01. 07. 1843, Karelin" (holotype LE!).

- Ferula nodiflora auct. non L.: Pall., 1776, Reise russ. Reich 3: 735.

Type: KAZAKHSTAN. "Inter collem arenosis, locis humidioribus, copiosissime supra fortelitium Iamyschewa, 06. 1771, Pallas".

Distribution in China: NW: Xinjiang Weiwuer A. R.

Distribution outside China: Kazakhstan, E Europe.

Ferula sinkiangensis Shen Kuanmien, 1975, Acta Phytotax. Sin. 13(3): 88, fig. 1.

Type: CHINA. "Xinjiang, Yinning, Baishidun, alt. 850 m, 13. 05-01. 06. 1973, Shen Kuanmien 069, 072, 093, 119" (syntypes XJBI!).

Distribution in China: NW: Xinjiang Weiwuer A. R. Endemic.

Ferula soongarica Pall. ex Spreng., 1820, in Roemer \& Schultes (eds.), Syst. Veg. (ed. 15 bis) 6: 598 . $\equiv$ Peucedanum aureum Spreng., 1824, Syst. Veg. (ed. 16) 1: 911, p.p.

Type: RUSSIA. "Siberia, Pallas" (holotype BM?; isotypes B-Willd - barcode B-W 05774 -01 0, M! - barcode M0173051).

= Peucedanum elatum Ledeb., 1829, Fl. Altaic. 1: 304 .

Type: KAZAKHSTAN. [Altai] "In declivibus herbedis ubique [Ad fl. Gramatucha], Ledebour 252" (lectotype LE!, designated by Vinogradova, 2005: 963; isolectotypes C - barcodes C1000844, C10008445, CGE!, HAL, P - barcodes P02272203, P02272204); "In declivibus apricis herbidis frequens, 1829, Bunge" (syntype LE!).

$=$ Ferula ehrenbergii H. Wolff, 1924, Repert. Spec. Nov. Regni Veg. 19: 310.

Type: RUSSIA. "Sibiria occidentalis, loco non indicato, Ehrenberg".

$=$ Ferula soongarica Pall. ex Spreng. var. arida Korovin, 1947,Gen. Ferula Monogr. Ill.: 71.

$=$ Ferula arida (Korovin) Korovin, 1963, in Pavlov (ed.), Fl. Kazakhst. 6: 411, tab. 57, fig. 2.

Type: KAZAKHSTAN. "In regione desertorum arida".

$=$ Ferula soongarica Pall. ex Spreng. subsp. mongolica V. M. Vinogr. et Kamelin, 1986, Novosti Sist. Vyssh. Rast. 23: 101.

$=$ Ferula mongolica $($ V. M. Vinogr. et Kamelin) V. M. Vinogr. et Kamelin, 1990, Novosti Sist. Vyssh. Rast. 27: 118.

Type: MONGOLIA. "Declivium montium in angustis Botchon, 18. 07. 1899, Klementz" (holotype LE!).

Distribution in China: NW: Xinjiang Weiwuer A. R.

Distribution outside China: Russia, Mongolia, Kazakhstan.

Ferula syreitschikowii Koso-Pol., 1922, Bot. Mater. Gerb. Glavn. Bot. Sada RSFSR 3(18): 71.

Type: KAZAKHSTAN. "In prov. Semiretschensk (Heptapotamia), distr. Kopal, in ripa fluminis Karatal inter loc. Kos-tugaj et Naiman-mula, salsa rimosa takyr dicta, 03. 06. 1913, Schischkin et Genina" (holotype LE!).

$=$ Ferula puberula Trautv., 1866, Bull. Soc. Imp. Naturalistes Moscou 39(2): 323, nom. illeg. (Art. 53), non Boiss. et Buhse (1860).

Type: KAZAKHSTAN. "Ad lacum Balchasch, 13. 06. 1841, Schrenk 349” (holotype LE!).

= Pastinaca olgae Regel et Schmalh., 1882, Izv. Obšč. Ljubit. Estestv. 34(2): 37.

Type: KAZAKHSTAN. "In Turkestatiae montibus Karak desertum Kisil-kum adjacentibus, 08. 05. 
1871, O. Fedtschenko" (lectotype LE!, designated by Vinogradova, 2002b: 139).

Distribution in China: NW: Xinjiang Weiwuer A. R.

Distribution outside China: Kazakhstan, Uzbekistan.

Ferula teterrima Kar. et Kir., 1842, Bull. Soc. Imp. Naturalistes Moscou 15: 363.

Type: KAZAKHSTAN. "In collibus lapidosis Songoriae prope fontem Sassyk-pastau, inter fluvios Ajagus et Lepsa, 06. 1841, Karelin et Kirilov 377" (lectotype LE!, designated by Korovin, 1951: 91; isolectotypes MW!, TK).

$=$ Ferula balchaschensis Bajtenov, 1970; Vestnik Akad. Nauk Kazak. SSR 7: 71.

Type: KAZAKHSTAN. "Regio Balchaschensis, in vallo ripario lacus arenoso schistose, 19. 06. 1938, Dmitrieva" (holotype AA!).

Distribution in China: NW: Xinjiang Weiwuer A. R.

Distribution outside China: Kazakhstan.

Ferula transiliensis (Herder) Pimenov, 1981, in Czerepanov (ed.), Sosud. Rast. SSSR: 21.

$\equiv$ Peucedanum transiliense Herder, 1866, Bull. Soc. Imp. Naturalistes Moscou 39(3): 78.

$\equiv$ Talassia transiliensis (Herder) Korovin, 1963, in Pavlov (ed.), Fl. Kazakhst. 6: 384, tab. 52, fig. 1.

Type: KAZAKHSTAN. "Kurmetty-Passe im Alatau Transiliensis, in einer Hohe von 7000" [Kurmesty pass, Alatau transil.], Semenov" (holotype LE!).

Distribution in China: NW: Xinjiang Weiwuer A. R.

Distribution outside China: Kazakhstan, Kirghizia, Tajikistan, Uzbekistan.

Ferulopsis mongolica Kitag., 1971, J. Jap. Bot. 46(9): 284, tab. 13, fig. 1.

Type: CHINA. "Mongolia Inter., in herbidis $1500 \mathrm{~m}$ alt. circa Pao-yuan (Ku-yuan) [HokushiMokyoo Hogen], 8-9. 09. 1942, Togashi 1940" (holotype TNS!).

Distribution in China: N: Neimenggu A. R. Endemic.

Foeniculum vulgare Mill., 1768, Gard. Dict. (ed. 8): Foeniculum N 1.

$\equiv$ Anethum foeniculum L., 1753, Sp. pl.: 263.

$\equiv$ Ligusticum foeniculum (L.) Crantz, 1767. Class. Umbell. Emend.: 82.

三Anethum rupestre Salisb., 1796, Prodr. Stirp. Chap. Allerton: 168, nom. illeg. $\equiv$ Meum foeniculum (L.) Spreng., 1813, Neue Schr. Naturf. Ges. Halle 2, 1 (Pl. Umb. Prodr.): 32.

$\equiv$ Foeniculum foeniculum (L.) H.Karst., 1882, Deut. Fl. 9: 837. nom. inval. (Art. 23.4).

$\equiv$ Selinum foeniculum (L.) E. H. L. Krause, 1904, in Sturm (ed.), Fl. Deutsch. (ed. 2) 12: 115.

$\equiv$ Seseli foeniculum (L.) Koso-Pol., 1916, Bull. Soc. Imp. Naturalistes Moscou, s.n. 29: 183.

Type: SOUTH EUROPE. "In Narbonne, Aremoriae, Maderae rupibus cretaceis, 106, Anethum 2" (lectotype BM-Cliff - barcodes BM000558381-5, designated by Jansen, 1981: 25).

$=$ Foeniculum officinale All., 1785, Fl. Pedem. 2: 25 .

Type: EUROPE, ITALY. "Circa Pinelorium. In comitatu Nicaeensi, et in provincia Uneliae".

= Anethum panmorium Roxb. ex Flem., 1810, Asiat. Res. 11: 156.

$=$ Foeniculum panmorium (Roxb.) DC., 1830, Prodr. 4: 142.

Type: INDIA. "Plants, cultivated in Bengal".

= Ozodia foeniculacea Wight et Arn., 1834, Prodr. Fl. Penins. Ind.Or. 1: 375.

Type: INDIA. "India Orientalis, Wight 1210" (holotype E! - barcode E00174688; isotypes NY, P).

=Foeniculum commune Bubani, 1900, Fl. Pyren 2: 372, "Feniculum", nom. illeg. (Art. 52.1)

= Foeniculum vulgare Mill. f. pauciradiatum Nábĕlek, 1923, Spisy Prir. Fak. Masarikovy Univ. 35: 127.

Type: EGYPT. "Arabia petraea, Wadi el Hsa inter El-Karac et Es-Sabak, inter rupes, alt. ca. 800 m, 16. 06. 1909, Nábělek 467" (holotype SAV).

Distribution in China: NW: Xinjiang Weiwuer A. R.; Tibet: Xizang A. R., Qinghai; N: Ningxia Huizu A.R., Shanxi, Hebei, Shandong; NE: Jilin; Centr.: Henan, Jiangsu, Hubei, Fujiang; SW: Guizhou, Yunnan; S: Guangdong.

Distribution outside China: Russia, Korea, Philippines, Indonesia, Malaysia, Thailand, Myanmar, Bangladesh, Bhutan, Nepal, India, Pakistan, Kazakhstan, Uzbekistan, Turkmenistan, Afghanistan, Iran, Azerbaijan, Georgia, Armenia, Turkey, Iraq, Qatar, UAE, Saudi Arabia, Yemen, Lebanon, Syria, Israel, Jordan, Egypt (Sinai), Cyprus, Greece (E Aegean Ils.). Europe, Africa, N. America, S. America, Australia and Oceania.

Glehnia littoralis (J. G. Cooper) F. Schmidt ex Miq., 1867 (I-VI), Ann. Mus. Bot. Lugduno-batavi 3: 61; 1867, Prolus. Fl. Jap.: 249..

$=$ Cymopterus littoralis J. G. Cooper in A. Gray [1859, Mem. Amer. Acad. 2, 6 (Bot. Jap.): 391, 
nomen]. 1860, Pacif. Railr. Rep. Parke, Bot. 12 (2): 62.

$=$ Phellopterus littoralis (J. G. Cooper) F. Schmidt, 1868, Mem. Acad. Imp. Sci. SaintPetersbourg (ser. 7) 12(2): 138.

Type: NORTH AMERICA, USA. "On the sands of the sea-shore at Shoalwater Bay [Washington Territory], 1854. Cooper" (holotype GH - barcode GH00075899).

Distribution in China: N: Hebei, Shandong; NE: Liaoning; Centr.: Jiangsu, Zhejiang, Fujiang; S: Taiwan, Guangxi Zhuang A.R., Guangdong.

Distribution outside China: Russia, Japan, Korea, N. America.

Haloselinum falcaria (Turcz.) Pimenov, 2012, Umbell. Russia: 300.

$\equiv$ Peucedanum falcaria Turcz., 1832, Bull. Soc. Imp. Naturalistes Moscou 5: 192.

Type: MONGOLIA. "In Mongolia Chinensis, 1831, Kuznetzov" (holotype LE!).

= Peucedanum salsugineum Krylov, 1903, Trudy Imp. S.-Peterburgsk. Bot. Sada 21(1): 8, tab. 5, fig. 1.

Type: RUSSIA. "In salsis deserti Tschujensis (Altai orientalis) [Altai, steppa Tschujensis, in angulo boreali-occidentali], 27-28. 07. 1901, Krylov" (lectotype TK!, designated by Gureeva et al., 2016; isolectotype LE!).

= Peucedanum pricei N. D. Simpson, 1913, J. Linn. Soc. Bot. 41: 419, tab. 23, fig. 1-3.

Type: MONGOLIA. "On the moist well-drained alluvial soil, not far from lakes and streams, Achit Nor, NW Mongolia [Acheet-nor, Mongolia], Price 108" (holotype K! - barcode K000685426).

Distribution in China: NW: Xinjiang Weiwuer A. R.; Tibet: Qinghai; N: Neimenggu A. R.

Distribution outside China: Russia, Mongolia.

Hansenia forbesii (H. Boissieu) Pimenov et Kljuykov, 2008, Willdenowia 38(1): 163.

$\equiv$ Notopterygium forbesii H. Boissieu, 1903, Bull. Herb. Boissier (2 ser.) 3(10): 840.

$\equiv$ Drymoscias forbesii (H. Boissieu) Koso-Pol., 1916, Bull. Soc. Imp. Naturalistes Moscou, s.n. 29: 118 , in adnot.

Type: CHINA. "Prov. Hupeh [Fang], 05. 1889, Henry 6629" (lectotype P!, designated here; isolectotypes K!, LE!, P!).

= Notopterygium franchetii $\mathrm{H}$. Boissieu, 1903, Bull. Herb. Boissier (2 sér.) 3(10): 839.

$=$ Drymoscias franchetii $(\mathrm{H}$. Boissieu) KosoPol., 1916, Bull. Soc. Imp. Naturalistes Moscou, s.n. 29: 118 , in adnot.
Type: CHINA. "Tan-Ken-Cheou, sousprefecture de Ta-Lin-Hoen, alt. 2500 m, 07. 1893, Farges 1268" (lectotype P!, designated here; isolectotypes P!); "Touan-Chen, sous-prefecture Ta-Li-Hoen, alt. 2200 m, , 07. 1898, Farges 1421" (syntypes P!).

= Angelica rubrivaginata $\mathrm{H}$. Wolff, 1926, Acta Horti Gothob. 2: 318.

Type: CHINA. 'Sze-ch'uan bor:: Ch'un-ch'e, Nadelwald, 3200 m. In silva abietina, 01. 08. 1922, H. Smith 4124" (lectotype UPS!, designated here; isolectotype GB! - barcode GB0048798).

= Angelica tsinlingensis Fu Kuntsun, 1981, Fl. Tsinling. 1(3): 420,461, fig. 358.

Type: CHINA. "Shenxi, Hwa-in Hsien, Hwashan, Ta-pai-yang-cha, alt. 1500 m, 22. 09. 1974, Fu Kuntsun 17242" (holotype WUK).

Distribution in China: NW: Gansu; Tibet: Qinghai; N: Neimenggu A. R., Ningxia Huizu A. R., Shaanxi, Shanxi; Centr.: Henan, Hubei; SW: Sichuan, Yunnan. Endemic.

Hansenia forrestii (H. Wolff) Pimenov et Kljuykov, 2008, Willdenowia 38(1): 166.

$\equiv$ Notopterygium forrestii $\mathrm{H}$. Wolff, Repert.Spec. Nov. Regni Veg. 27: 325.

Type: CHINA. "Yunnan, Mekong-Salwin divide. Lat. $28^{\circ} 10^{\prime}, 10000^{\prime}$, open stony pasture on the margins of the thickets and streams, 09. 1914, Forrest 13342" (lectotype E!, designated here; isolectotypes BM!, PE!).

Distribution in China: SW: Sichuan, Yunnan. Endemic.

Hansenia oviformis (Shan Renhwa) Pimenov et Kljuykov, 2008, Willdenowia 38(1): 166.

三Notopterygium oviforme Shan Renhwa, 1943, Sinensia 14: 112, fig. 1.

$\equiv$ Notopterygium forbesii $\mathrm{H}$. Boissieu var. oviforme (Shan Renhwa) Chang Hotseng, 1975, Acta Phytotax. Sin. 13(3): 85.

$\equiv$ Notopterygium forbesii $\mathrm{H}$. Boissieu subsp. oviforme (Shan Renhwa) Pu Fating, 2000, Acta Phytotax. Sin. 38(5): 433.

Type: CHINA. "Szechuan, Western China Sci. Inst. 1382”.

= Ligusticum litangense Pu Fating, 1991, Acta Phytotax. Sin. 29(6): 534.

Type: CHINA. "Sichuan: Litang, Kalatz Mountain, alt. $4300 \mathrm{~m}$, in the alpine bush meadow, 20. 09. 1984, Pu Fating 374" (holotype CDBI!; isotype NAS!).

Distribution in China: N: Shaanxi; SW: Sichuan. Endemic. 
Hansenia pinnatiinvolucellata ( $\mathrm{Pu}$ Fating et Wang Youping) Pimenov et Kljuykov, 2008, Willdenowia 38(1): 169.

$=$ Notopterygium pinnatiinvolucellatum $\mathrm{Pu}$ Fating et Wang Youping, 1994., J. Sichuan Univ., Nat. Sci. ed.. 31(3): 386.

Type: CHINA. "Sichuan: Xiaoling, Balang Mountain, alt. ca. $3400 \mathrm{~m}$, among schrubs in grassland at the edge of mountain coniferous forests, 26. 09. 1990, Pu Fating, Wang Youping et al. 90061" (holotype CDBI).

Distribution in China: SW: Sichuan. Endemic.

Hansenia weberbaueriana (Fedde ex H. Wolff) Pimenov et Kljuykov, 2008, Willdenowia 38(1): 169.

三Ligusticum weberbauerianum Fedde ex H. Wolff, 1930, Repert. Spec. Nov. Regni Veg. 27: 312.

$\equiv$ Notopterygium weberbauerianum (Fedde ex H. Wolff) Pimenov et Kljuykov, 1999, Feddes Repert. 110(7-8): 485.

Type: CHINA. "Central-Kansu: Lien hoa shan, forests, 10000 ft., 14-20.07.1925, Rock 12727”, (lectotype GH!, designated here; isolectotypes BM! barcode BM000885358, E! - barcode E00002395, GH - barcode GH00076822, K!, NAS!).

= Ligusticum pilgerianum H. Wolff, 1930, Repert. Spec. Nov. Regni Veg. 27: 307.

Type: CHINA. "Sze-chuan, reg. bor.: Dongrergo, in prato fruticoso-herboso, ca. $4200 \mathrm{~m}$ s.m., 08. 08. 1922, H. Smith 3544" (lectotype UPS!, designated here; isolectotype PE!).

= Notopterygium incisum Ting Chihchi et Chang Hotseng, 1975, Acta Phytotax. Sin. 13(3): 86.

Type: CHINA. "Qinghai, Qumarleb, Qu Malai et Liu Shangwu 863" (holotype HNWP).

Distribution in China: NW: Gansu; Tibet: Xizang A. R., Qinghai; N: Shaanxi; Centr.: Henan; SW: Sichuan. Endemic.

Haplosphaera himalayensis Ludlow, 1976, Bull. Brit. Mus. (Nat. Hist.), Bot. 5(5): 276.

Type: CHINA. "SE. Tibet, Kongbo province, Miraha, Nyang Chu, 3900 m, stony situations in clearings in Abies forest, 17. 08. 1938, Ludlow, Sherriff et Taylor 6087" (holotype E! - barcode E00265231, designated here; isolectotypes E barcodes E00265232, E00265233).

Distribution in China: Tibet: Xizang A. R., Qinghai.

Distribution outside China: Bhutan, Nepal, India.
Haplosphaera phaea Hand.-Mazz., 1920, Anz. Akad. Wiss. Wien 57: 143.

Type: CHINA. "Prov. Yunnan bor-occid.: in silvis abietinis inter Bodo et Alo ad austro-orient. oppidi Dschungdien (Chungtien), ca. $3800 \mathrm{~m}$, 08. 08. 1914, Handel-Mazzetti 4596" (lectotype WU! - barcode WU0025964, designated here; isolectotypes E! - barcode E00002389, FI!,WU! barcode WU0025965).

Distribution in China: SW: Sichuan, Yunnan. Endemic.

Harrysmithia franchetii (M. Hiroe) Sheh Menglan, 2004, Acta Phytotax. Sin. 42(6): 562.

ECarum dissectum Franch., 1894, Bull. Annuel Soc. Philom. Paris (sér. 8) 6: 123, nom. illeg. (Art. 53.1), non Baill. (1879).

三 Carum franchetii M. Hiroe, 1979, Umbelliferae World: 871.

三 Harrysmithia dissecta $\mathrm{H}$. Wolff ex Shan Renhwa [1984, in Wu Zhengyi, Index Fl. Yunnan. 1: 911, nom. illeg.] 1985, Fl. Reipubl. Popularis Sin. 55(2): 135, tab. 55, fig. 1-6.

Type: CHINA. "[Yunnan] Les bois de Ta-longtan, près de Ta-pin-tze, 17. 09. 1888, Delavay 3253" (lectotype P! - barcode P00752754, designated here; isolectotypes GH - barcodes GH00062635, GH00283232, K! - barcode K000695723, NY barcode NY00405777, P! - barcodes P00752755, P00752756, UC).

Distribution in China: SW: Yunnan. Endemic.

Harrysmithia heterophylla $\mathrm{H}$. Wolff, 1926, Acta Horti Gothob. 2: 311.

Type: CHINA. "Sze-ch'uan bor-occid.: Drogochi ad Tsagavageu, $3300 \mathrm{~m}, 16.09 .1922$, H. Smith 4792" (lectotype UPS!, designated here; isolectotypes BM! - barcode BM000885464, GB!, LD!).

Distribution in China: Tibet: Xizang A. R.; SW: Sichuan. Endemic.

Heracleum bivittatum H. Boissieu, 1903, Bull. Herb. Boissier (2 sér.) 3(10): 855.

$\equiv$ Tetrataenium bivittatum (H. Boissieu) Manden., 1986, Zametki Sist. Geogr. Rast. 41: 45.

Type: CHINA. "Yunnan, montagne Menguen, 13. 12. 1897, Bons d'Anty" (lectotype P! - barcode P02284843, designated here); "Au pied de LouangOuang-Chou, 09.1895, Delavay 6904” (syntype P! barcode 00245505).

= Peucedanum angelicoides Kretschmer ex $\mathrm{H}$. Wolff, 1930, Repert. Spec. Nov. Regni Veg. 27: 313. 
Type: CHINA. "[Yunnan] Houang, G'ao-pa. 1917, Cavalerie 4320" (holotype P!).

Distribution in China: SW: Sichuan, Guizhou, Yunnan; S: Guangxi Zhuang A.R.

Distribution outside China: Vietnam, Laos, Thailand.

Heracleum candicans Wall. ex DC., 1830, Prodr. 4: 192.

$\equiv$ Tetrataenium candicans (Wall. ex DC.) Manden., 1986, Zametki Sist. Geogr. Rast. 41: 44.

Type: INDIA. "[In India septentr. ad Deyradoon et Kamaon] Kumaon, Blinkworth [573]" (lectotype G-DC!, designated by Watson, 1998: 373; isolectotypes BM!-photo, CAL, K-WALLICH! - barcode K000075504, K001111316, LE!); "Deyra Dhoon, 1825, Wallich" (syntype K-WALLICH! - barcode K000075503).

= Heracleum cinereum Lindl., 1835, in Royle, Ill. Bot. Himal. Mts.: 232.

Type: INDIA. "Mussooree, Royle" (holotype CGE!).

= Heracleum hypoleucum Vis., 1845, Linnaea 18: 512.

Type: NEPAL. "In Nepaulia (Semine habui ab ill. Societ. horticola Londinense, et ab H. Hafnense)".

- Heracleum lanatum auct. non Michx.: Aswal \& Mehrotra, 1994, Fl. Lahaul-Spiti: 286.

Distribution in China: Tibet: Xizang A. R., Qinghai; SW: Sichuan, Yunnan.

Distribution outside China: Myanmar, Bhutan, Nepal, India, Pakistan.

Heracleum dissectifolium Fu Kuntsun, 1981, Fl. Tsinling. 1(3): 464, 431, fig. 366.

Type: CHINA. "Kansu: Tan-chang Hsien, Hwaer-po, alt. 3260 m, 13. 08. 1969, Y. P. Shu 1887" (holotype WUK).

Distribution in China: NW: Gansu; N: Ningxia Huizu A.R.; SW: Sichuan. Endemic.

Heracleum dissectum Ledeb., 1829, Fl. Altaic. 1: 301 .

$\equiv$ Pastinaca dissecta (Ledeb.) Koso-Pol., 1916, Bull. Soc. Imp. Naturalistes Moscou, s.n. 29: 113, nom. illeg. (Art. 53.1), non Vent. (1803).

$\equiv$ Heracleum sphondylium L. f. dissectum (Ledeb.) H.Ohba, 1994, J. Jap. Bot. 69(2): 119.

Type: KAZAKHSTAN. "In pratis prope pagum Tschetschulicha, metallifodinam Riddersk et alibi [Prope Tschetschulicha], Ledebour 580, 242" (lectotype LE, designated by Vinogradova, 2005: 962; isolectotype $\mathrm{P} !$ - barcode P02272123).
= Heracleum barbatum Ledeb., 1829, Fl. Altaic. 1: 300 .

= Pastinaca barbata (Ledeb.) Koso-Pol., 1916, Bull. Soc. Imp. Naturalistes Moscou, s.n. 29: 113.

= Heracleum dissectum Ledeb. var. barbatum (Ledeb.) Krylov, 1904, Fl. Alt. Tomsk. Gub. 3: 534.

Type: RUSSIA. "[Altai] In pratis montanis [Riv. Pichtovka prope Buchtarminsk] 20. 06. 1826, Meyer 295" (lectotype LE, designated by Vinogradova, 2005: 962).

= Heracleum microcarpum Franch., 1884, Nuov. Arch. Mus. Hist. Nat. (sér. 2) 6 (Pl. David. 1): 144.

Type: CHINA. "Mongolie, Gehol, dans les hautes prairies de Nantachan, 08. 1864, David 2092" (lectotype P - barcode P02272124, designated here; isolectotypes P - barcodes P02516466, P04101987, US).

- Heracleum moellendorffii auct. non Hance: Gorovoj, 1966, Umbell. Prim. Priam.: 212, tab. $146-148$

Distribution in China: NW: Xinjiang Weiwuer A. R.; N:Neimenggu A. R., Hebei; NE: Heilongjiang, Jilin, Liaoning.

Distribution outside China: Russia, Korea, Mongolia, Kazakhstan, Kirghizia, Tajikistan, Uzbekistan.

Heracleum fargesii $\mathrm{H}$. Boissieu, 1903, Bull. Herb. Boissier (2 sér.) 3(10): 853.

Type: CHINA. "Su-Tchuen oriental, à TchenKéou-Tin, Farges" (lectotype P! - barcode P03810953, designated here; isolectotypes B! barcode B $100146583, \mathrm{~K}, \mathrm{P}$ ! - barcodes P00245525, P00245526, P03810954, PE!).

Distribution in China: SW: Sichuan. Endemic.

Heracleum forrestii H. Wolff, 1934. Repert. Spec. Nov. Regni Veg. 33: 75.

Type: CHINA. "Yunnan, West China. Chungtien plateau. Lat. $27^{\circ} 30^{\prime}$. Open rocky situations on the margins of thickets, 08. 1914, Forrest 13115" (holotype E!).

Distribution in China: SW: Sichuan, Yunnan. Endemic.

Heracleum franchetii M. Hiroe, 1979, Umbelliferae World: 1749.

$\equiv$ Heracleum acuminatum Franch., 1894, Bull. Annuel Soc. Philom. Paris (sér. 8) 6: 144, nom. illeg. (Art. 53.1), non Schleich. (1821).

Type: CHINA. "Yun-nan, in umbrosis montis La-long-tong, supra collum Yen-tze-hay, alt. 3500 m, 18. 07. 1889, Delavay 3982" (lectotype P! - 
barcode P00245490, designated here; isolectotypes P! - barcodes P00245487, P00245488, P04330945, P03810945, P03810946); "Yun-nan, in collibus montis Tsang-Chan, alt. $4000 \mathrm{~m}, 29$. 08. 1889, Delavay 4059" (syntype P!); "Yun-nan, in monte Ma-eul-chan, 10. 09. 1889. Delavay" (syntype P! barcode P00245486).

Distribution in China: NW: Gansu; Tibet: Qinghai; Centr.: Hubei; SW: Sichuan, Yunnan. Endemic.

Heracleum hemsleyanum Diels, 1900, Bot. Jahrb. Syst. 29 (3-4) (Fl. Centr. China): 503.

Type: CHINA. "Central-China, O [Hsingshan and Fang], Henry 6469" (lectotype K! - barcodes K000075542, K000075544, designated here; isolectotype E - barcodes E00168002, E00168003).

Distribution in China: Centr.: Henan, Hubei; SW: Sichuan. Endemic.

Heracleum henryi H. Wolff, 1934, Repert. Spec. Nov. Regni Veg. 33: 76.

Type: CHINA. "Yunnan, 5000' s.m., Henry 12486B" (holotype E! - barcode E00002772).

Distribution in China: SW: Yunnan. Endemic.

Heracleum kansuense Diels, 1906, Repert. Spec. Nov. Regni Veg. 2(18): 66.

Type: CHINA. "China occidentalis: prov. Kansu: Hsi ming fu, 1904, Filchner 21".

Distribution in China: NW: Gansu. Endemic.

Heracleum kingdonii H. Wolff, 1934, Repert. Spec. Nov. Regni Veg. 33: 76.

Type: CHINA. "Yunnan, Laktang, $9000 \mathrm{ft}$. $26^{\circ} 10^{\circ}$ N, $98^{\circ}$ E, 27. 06. 1919, Kingdon-Ward 3278" (holotype E!).

- Heracleum burmanicum auct. non Kurz: Wang Tiehseng, 1992,in Shen Renhwa \& Sheh Menglan (eds.), Fl. Reipubl. Popularis Sin. 55(3): 199, tab. 87, fig. 7-8.

Distribution in China: Tibet: Xizang A. R.; SW: Guizhou, Yunnan; S: Guangxi Zhuang A. R. Endemic.

Heracleum likiangense $\mathrm{H}$. Wolff, 1933, Repert. Spec. Nov. Regni Veg. 33: 78.

Type: CHINA. "Yunnan: Yangtze watershed, prefectural district of Likiang, eastern slopes of Likiang Snow Range, alpine meadows, 13500', 08. 07. 1922, Rock 4957" (holotype US).

Distribution in China: SW: Yunnan. Endemic.
Heracleum moellendorffii Hance, 1878, J. Bot.

16: 12 .

$\equiv$ Heracleum barbatum Ledeb. subsp. moellendorffii (Hance) M. Hiroe, 1949, Acta Phytotax. Geobot. 14(1): 4 .

$\equiv$ Heracleum lanatum Michx. subsp. moellendorffii (Hance) H. Hara, 1954, Enum. Sperm. Jap. 3: 310 .

Type: CHINA. "In m. Po Hua shan Chinae bor., 06. 10. 1874, Moellendorff 19197" (lectotype BM!, designated here; isolectotype LE!).

= Heracleum morifolium $\mathrm{H}$. Wolff, 1926, Acta Horti Gothob. 2: 326.

Type: CHINA. "Chili: Hsiao-wu-tai-shan, Yang-kia-p'ing, Tung-lin, am Bache, $1200 \mathrm{~m}$ [Ad rivulum in valle herbosa], 05. 09. 1921, H. Smith 112" (lectotype UPS!, designated here); "Chili: Hsiao-wu-tai-shan, Yang-kia-p'ing. 1000 m. In silva frondosa, 09. 1921, H. Smith 438" (syntypes GB! barcode GB-0048812, UPS!).

= Heracleum inperpastum Koidz. [1938,Acta Phytotax. Geobot. 7: 267, nom inval., in Japanese (Art. 39.1)], 1939, Acta Phytotax. Geobot. 8: 53.

Type: JAPAN. "Japonia, prov. Tamba, Johwoh., 02. 06. 1929, Araki 9709” (holotype KYO!).

= Heracleum tabuchii Y. Akasawa, 1987, Phytatax. Rep. Akasawa Bot. Lab. 1(1): 7.

Type: JAPAN. "In Fagi-silvis montis Ishidate circiter 1659 m supra mare, 10. 08. 1986, Tabuchi".

Distribution in China: NW: Gansu; N: Neimenggu A. R., Shaanxi, Hebei, Shandong; NE: Heilongjiang, Jilin, Liaoning; Centr.: Henan, Jiangsu, Anhui, Hubei, Hunan, Jiangxi, Zhejiang; SW: Guizhou.

Distribution outside China: Korea, Japan.

Heracleum nepalense D. Don, 1825, Prodr. Fl. Nepal.: 185.

$\equiv$ Tetrataenium nepalense (D. Don) Manden., 1959, Trudy Tbilis. Bot. Inst. 20: 18.

Type: NEPAL. "Mont. alt. Nepalia, 06.1821, Wallich [576]" (lectotype BM! - barcode BM000521533, designated by Watson, 1998: 374; isolectotypes CAL, E - barcodes E00002938, E00002939, K - barcodes K000075500, K000075501, K000075502, K-WALLICH! barcodes K001111321, K001111323, LD! - barcode LD1221930, LE!, M! - barcode M0173037).

= Heracleum nepalense D. Don var. bivittatum C. B. Clarke, 1979, in Hooker f. (ed.), Fl. Brit. India 2: 714 .

Type: INDIA/BHUTAN. "Sikkim and Bhotan". 
$=$ Heracleum nyalamense Shan Renhwa et Wang Tiehseng, 1980, Acta Phytotax. Sin. 18(3): 378.

Type: CHINA. "Xizang: Nyalam Xian, alt. 2300 m, 13. 08. 1972, Tibet Exped. Chin. Med. 1055" (holotype PE).

Distribution in China: Tibet: Xizang A. R.; SW: Yunnan.

Distribution outside China: Myanmar, Bhutan, Nepal, India.

Heracleum obtusifolium Wall. ex DC., 1830, Prodr. 4: 191.

$\equiv$ Tetrataenium obtusifolium (Wall. ex DC.) Manden., 1991, Zametki Sist. Geogr. Rast. 42: 13.

$\equiv$ Heracleum candicans Wall. ex DC. var. obtusifolium (Wall. ex DC.) Pu Fating et M. F. Watson, 2004, Acta Phytotax. Sin. 42(6): 562.

Type: NEPAL. "In Nepalia, 1821, Wallich [577]" (lectotype G-DC! - barcode G00324695, designated by Watson, 1998: 374; isolectotypes CAL, K-WALLICH! - barcode K001111324).

Distribution in China: Tibet: Xizang A. R.; SW: Sichuan, Yunnan.

Distribution outside China: Bhutan, Nepal, India.

Heracleum oncosepalum (Hand.-Mazz.) Pimenov et Kljuykov, 2003, Willdenowia 33(1): 132, fig. 7.

$\equiv$ Angelica oncosepala Hand.-Mazz., 1933, Symb. Sin. 7(3): 726.

Type: CHINA. "Yunnan bor.-occid.: in montium inter fluvios Langsang-djiang (Mekong) et Ludjiang (Salween), ca. 28 9', regione alpinis in cen. spite inter lacum et jugum Yigoru, 4200-4300 m, 06. 08. 1916, Handel-Mazzetti 9713" (lectotype WU! - barcode WU0025988, designated here; isolectotypes E! - barcode E00002379, W!).

Distribution in China: SW: Yunnan. Endemic.

Heracleum oreocharis H. Wolff, 1933, Repert. Spec. Nov. Regni Veg. 33: 77.

Type: CHINA. "Yunnan: Yangtze watershed, prefectural district of Likiang, eastern slopes of Likiang Snow Range, 12500-14000' s.m., 13. 08. 1922, Rock 5801" (syntypes E!, US).

Distribution in China: SW: Yunnan. Endemic.

Heracleum pinnatum C. B. Clarke, 1879, in Hooker f. (ed.), Fl. Brit. India 2: 712.

$\equiv$ Tetrataenium pinnatum (C. B. Clarke) Manden., 1991, Zametki Sist. Geogr. Rast. 42: 12.

Type: INDIA. "Tibet occ. [Kashmir northwards to the Karakorum], Piti, alt. 10000-13000 ft., 05.
09. 1847, Thomson 14" (lectotype K! - barcode K000685813, designated here; isolectotypes BM!, CAL, E - barcode E00002942, G!, K! - barcode K000685810), LE!, M! - barcodes M0173034, M0173035, NY - barcode NY00405968, P barcode P00753133, UC, W!); "Tibet occ., Sassar, 15000', 15. 08. 1848, Thomson" (syntype K!); "Le [Leh to Kaltse], 12. 07. 1848, Thomson" (syntypes BM - barcode BM000885313, K! - barcode K000685809); “Tibet, prov. Nubra, Liagtsung to Panamuk, left side of the Nubra valley, 24. 07. 1856, Thomson" (syntype BM! - barcode BM000944792).

$=$ Heracleum subtomentellum $\mathrm{Wu}$ Zhengyi et Sheh Menglan, 1991, Acta Bot. Yunnan. 13(3): 274.

Type: CHINA. "Xizang: Ali, alt. 4400 m, 05. 09. 1976, Qinghai-Xizang Exped. 76-9163" (holotype KUN).

Distribution in China: Tibet: Xizang A. R.

Distribution outside China: India, Pakistan.

Heracleum rapula Franch., 1894, Bull. Annuel Soc. Philom. Paris (sér. 8) 6: 145.

Type: CHINA. "Yun-nan, ad collum Pi-iouse supra Tapin-tze, alt. $2000 \mathrm{~m}, 15.10 .1886$, Delavay 2516" (lectotype P! - barcode P00245538, designated here; isolectotype P! - barcodes P00245537, P002455379).

Distribution in China: SW: Yunnan. Endemic.

Heracleum scabridum Franch., 1894, Bull. Annuel Soc. Philom. Paris (sér. 8) 6: 145.

Type: CHINA. "Yun-nan: in pratis ad Mo-soyn [Lan Kong], 2200 m, 30.05.1884, Delavay 676" (lectotype P! - barcode P00245549, designated here; isolectotypes $\mathrm{K}$ ! - barcode K000075531, P! barcode P00245548, P00245550).

Distribution in China: Centr.: Henan; SW: Sichuan, Yunnan. Endemic.

Heracleum schansianum Fedde ex H. Wolff, 1934, Repert. Spec. Nov. Regni Veg. 33: 78.

Type: CHINA. "Shansi: 1800 m, 03. 10. 1924, H. Smith 7632" (lectotype UPS!, designated here; isolectotype GB?).

Distribution in China: N: Shanxi. Endemic.

Heracleum souliei H. Boissieu, 1903, Bull. Herb. Boissier (2 sér.) 3(10): 852.

Type: CHINA. "Ta-Tsien-Lou, princ. de Kia-La [Thibet oriental, Tongolo (principalité de Kuala)], 20. 07. 1893, Soulié 299" (lectotype P! - barcode P00245556, designated here; isolectotypes MPU - 
barcode MPU021077, P! - barcodes P00245554, P00245555, P00245557).

Distribution in China: SW: Sichuan. Endemic.

Heracleum stenopteroides Fedde ex H. Wolff, 1934, Repert. Spec. Nov. Regni Veg. 33: 79.

Type: CHINA. "Yunnan: Divide between the Shweli and valleys; Lat. 20¹'. Alt. 6-7000'. Amongst scrub, 05. 1912, Forrest 7911" (holotype E! - barcodes E00002740, E00002741).

Distribution in China: SW: Yunnan. Endemic.

Heracleum stenopterum Diels, 1912, Notes Roy. Bot. Gard. Edinburgh 5: 291.

Type: CHINA. "[Yunnan] Open, shady situations in shady pine forests on the eastern flank of the Likiang Range. Lat. $27^{\circ} 15^{\prime}$. Alt. 10-11000 ft.,15. 08. 1906, Forrest 2847" (lectotype E! barcodes E00002763, E00002764, designated here; isolectotype BM - barcode BM000826892).

Distribution in China: SW: Sichuan, Yunnan. Endemic.

Heracleum sublineare C. B. Clarke, 1879, in Hooker f. (ed.), Fl. Brit. India 2: 713.

$\equiv$ Tetrataenium sublineare (C. B. Clarke) Manden. ex Farille et al., 1985, Candollea 40(2): 562.

Type: INDIA. "Sikkim, Lachen, alt. 1000013000 ft., 30. 07. 1849. Hooker Pastinaca 4" (lectotype K - barcodes K000075507, K000075508, designated by Watson, 1998: 374; isolectotype P! barcode P00753127).

Distribution in China: Tibet: Xizang A. R.

Distribution outside China: Bhutan, Nepal, India.

Heracleum tiliifolium H. Wolff, 1933, Repert. Spec. Nov. Regni Veg. 33: 80.

Type: CHINA. "Kiangsi: Lu Shan, damp brushy ground; Temple in the clouds, 30. 08. 1922, Steward 2677" (lectotype E! - barcode E00002765, designated here; isolectotype K! - barcode K000075539).

Distribution in China: Centr.: Anhui, Hunan, Jiangxi. Endemic.

Heracleum vicinum H. Boissieu, 1903, Bull. Herb. Boissier (2 sér.) 3(10): 853.

Type: CHINA. "Su-Tchuen oriental, à TchenKeou-Tin, Farges 265" (lectotype P! - barcode P00245566, designated here; isolectotypes K! barcode K000075538, P! - barcodes P00245567,
P00245568, P00245569, P00245570, P03810947, P03810948).

Distribution in China: SW: Sichuan. Endemic.

Heracleum wenchuanense $\mathrm{Pu}$ Fating et $\mathrm{He}$ Xingjin, 1993, Acta Phytotax. Sin. 31(4): 368, fig. 1.

Type: CHINA. "Sichuan: Wenchuan, Balang Mt., alt. apout $3500 \mathrm{~m}$ at the edge of forests, 26. 09. 1990, Pu Fating et He Xingjin 9063" (holotype CDBI!; isotype NAS).

Distribution in China: SW: Sichuan. Endemic.

Heracleum wolongense $\mathrm{Pu}$ Fating et He Xingjin, 1993, Acta Phytotax. Sin. 31(4): 372, fig. 2.

Type: CHINA. "Sichuan: Wenchuan, Wolong, Shawang, in schrubs or grassland, alt. $2200 \mathrm{~m}, 28$. 07. 1990, He Xingin H-017" (holotype CDBI!).

Distribution in China: SW: Sichuan. Endemic.

Heracleum yungningense Hand.-Mazz., 1933, Symb. Sin. 7(3): 729.

Type: CHINA. "Prov.Yunnan bor.-occid.: prope pagum Yungning in regione temperatae herbosis saltus trans jugum Fongkou, 3225 m, 16. 07. 1915, Handel-Mazzetti 7050" (lectotype WU! - barcodes WU0025958, WU0025959, designated here; isolectotype $\mathrm{W}$ !).

Distribution in China: Centr.: Hubei; SW: Sichuan, Yunnan. Endemic.

Heracleum yunnanense Franch., 1894, Bull. Annuel Soc. Philom. Paris (sér. 8) 6: 143.

$\equiv$ Tetrataenium yunnanense (Franch.) Manden. ex Pan Zehui, 1997, in Wu Zhengyu et al. (ed.), Fl. Yunnan. 7: 638, tab. 208, fig. 6-9.

Type: CHINA. "Yun-nan, in pascuis ad collum montis Lopin-chan, 31. 08. 1888, Delavay 3206" (lectotype P! - barcode P03227159, designated here; isolectotype P! - barcode P03227158).

Distribution in China: SW: Yunnan Endemic.

Hyalolaena bupleuroides (Schrenk ex Fisch. et C. A. Mey.) Pimenov et Kljuykov, 1982, Bot. Zhurn. (Leningrad) 67(7): 887, fig. 9, map 1, 2.

$\equiv$ Carum bupleuroides Schrenk ex Fisch. et C. A. Mey., 1845, Bull. Phys.-Math. Acad. Petersb. 3: 305.

इ Hymenolyma bupleuroides (Schrenk ex Fisch. et C. A. Mey.) Korovin, 1948, Not. Syst. Herb. Inst. Bot. \& Zool. Acad. Sci. Uzbekistan 12: 51.

Type: KAZAKHSTAN. "Songaria, am Balchasch, 16. 06. 1843, Schrenk 357" (holotype $\mathrm{LE}$ !; isotype MANCH!). 
A. R.

Distribution in China: NW: Xinjiang Weiwuer

Distribution outside China: Kazakhstan, Kirghizia, Tajikistan.

Hyalolaena issykkulensis Pimenov et Kljuykov, 1990, Bot. Zhurn. (Leningrad) 75(1): 92, fig. 3, 4.

Type: KIRGHIZIA. "in declivi boreali jugi Terskei Alatau, in valle fl. Chon-Kysylsu, 14. 07. 1974, Pimenov et Kljuykov 907" (holotype LE!; isotype MW!).

A. R.

Distribution in China: NW: Xinjiang Weiwuer

Distribution outside China: Kirghizia.

Hyalolaena trichophylla (Schrenk) Pimenov et Kljuykov, 1982, Bot. Zhurn. (Leningrad) 67(7): 887, fig. 8, map 1, 1 .

$\equiv$ Carum trichophyllum Schrenk, 1841, Enum. P1. Nov. 1: 61.

$\equiv$ Conopodium trichophyllum (Schrenk) Korovin, 1924, Bull. Sredne-Asiatsk. Gosud. Univ. 7, Suppl. (Sched. Herb. Fl. As. Med. 1-2): 25.

$\equiv$ Bunium trichophyllum (Schrenk) H. Wolff, 1927, in Engler (ed.), Pflanzenreich 90(IV, 228): 210.

$\equiv$ Hymenolyma trichophyllum (Schrenk) Korovin, 1948, Not. Syst. Herb. Inst. Bot. \& Zool. Acad. Sci. Uzbekistan 12: 31.

Type: KAZAKHSTAN. "In desertis versus fl. Aksu [Songaria, Aksu], 10. 06. 1840, Schrenk" (lectotype LE!, designated by Vinogradova, 1997a: 99; isolectotype P! - barcode P03237366).

= Seseli scariosum Kar. et Kir., 1842, Bull. Soc. Imp. Naturalistes Moscou 15: 361.

= Hymenolyma scariosum (Kar. et Kir.) Korovin, 1948, Not. Syst. Herb. Inst. Bot. \& Zool. Acad. Sci. Uzbekistan 12: 31.

Type: KAZAKHSTAN. "In argillosis Songoriae ad rivulum Ai, 1841, Karelin et Kirilov 1526" (syntypes LE!, MW!, P - barcode P02496402, TK); "Ad fl. Lepsa circa radicem montium Alatau, 06. 1841, Karelin et Kirilov 1526" (lectotype LE!, designated by Vinogradova, 2002b: 135).

$=$ Korovinia goloskokovii Bajtenov, 1977, Bot. Mater. Gerb. Inst. Bot. Akad. Nauk Kazakhsk. SSR 10: 37 .

Type: KAZAKHSTAN. "Regio ripae dextrae adjacens fl. Ili inter Panfinov and Chorgos, in demissis loessaceis ad marginem arenis Muyunkum, 04. 07. 1971, Goloskokov" (holotype AA!; isotypes AA!, LE!).

Distribution in China: NW: Xinjiang Weiwuer A. R.
Distribution outside China: Kazakhstan, Kirghizia, Turkmenistan.

Hydrocotyle batrachium Hance, 1862, Ann. Sci. Nat. (Paris), Bot. (4 sér.) 18: 220.

$\equiv$ Hydrocotyle rotundifolia Roxb. ex DC. var. batrachium (Hance) Cherm., 1921, Bull. Soc. Bot. France 68: 508.

$\equiv$ Hydrocotyle sibthorpioides Lam. var. batrachium (Hance) Hand.-Mazz. ex Shan Renhwa, 1936, Sinensia 7: 480.

Type: CHINA. "In locis irriguis ad Whampoam, Hance 7431" (holotype BM! - barcode BM000798552).

= Hydrocotyle formosana Masam., 1930, J. Soc. Trop. Agric. Taiwan 2: 51.

Type: CHINA. "Formosa, Taihoku, 06. 1930, Masamune".

Distribution in China: Centr.: Hunan, Hubei, Jiangxi, Fujiang; S: Taiwan, Guangdong.

Distribution outside China: Japan (Ryukyu Ils.), Vietnam.

Hydrocotyle benguetensis Elmer, 1909, Leafl. Philipp. Bot. 2: 628.

Type: PHILIPPINES. "Baguio, Province of Benguet, Luzon, 03. 1907, Elmer 8903".

= Hydrocotyle ranunculifolia Ohwi, 1933, Acta Phytotax. Geobot. 2: 151.

Type: CHINA. "Formosa, m. Shichiseizan in Taihokushi, 17. 05. 1933, Ohwi 2018" (lectotype $\mathrm{KYO}$ !, designated here; isolectotypes $\mathrm{K}$ ! - barcode K000686214, TI!, TNS!).

Distribution in China: S: Taiwan.

Distribution outside China: Japan, Philippines.

Hydrocotyle changanensis Du Xichun et Ren Yi, 2010, Ann. Bot. Fennici, 47(5): 404.

Type: CHINA. "China. Chaanxi Province: Chang'an district, Xi'fn, 400-600 m, in damp or slightly most areas. 08. 02. 2005, Du Xichun 20050629" (holotype SANU; isotype SANU).

Distribution in China: N: Shaanxi. Endemic.

Hydrocotyle craibii H. Eichler, 1987, Feddes Repert. 98(3-4): 146.

$\equiv$ Hydrocotyle javanica Thunb. var. chinensis Dunn ex Shan Renhwa et Liou Shoulu, 1964, Acta Phytotax. Sin. 9(2): 129.

$\equiv$ Hydrocotyle shanii Boufford, 1990, Acta Phytotax. Sin. 28(4): 331.

$\equiv$ Hydrocotyle burmanica Kurz subsp. chinensis (Dunn ex Shan Renhwa et Liou Shoulu) Pu Fating, 
1993, in Wang Wentsai (ed.), Vasc. Pl. Hengduan Mount.: 1277, comb. inval.

$\equiv$ Hydrocotyle burmanica Kurz subsp. craibii (H. Eichler) Wu Zhengyi et Pu Fating, 1998, Novon 8(1): 70.

$\equiv$ Hydrocotyle hookeri (C. B. Clarke) Craib subsp. chinensis (Dunn ex Shan Renhwa et Liou Shoulu) M. F. Watson et Sheh Menglan, 2004, Acta Phytotax. Sin. 42(6): 562.

Type: CHINA. "Yunnan: Mengzi, Mts. to 8500', Henry 10244" (holotype K! - barcode K000686213).

- Hydrocotyle chinensis auct. non L.: Craib, 1911, Kew Bull. 1911: 58.

Distribution in China: Centr.: Hubei, Hunan, Zhejiang; SW: Sichuan, Guizhou, Yunnan; S: Guangdong.

Distribution outside China: Vietnam.

Hydrocotyle dichondroides Makino, 1910, Bot. Mag. (Tokyo) 24: 242.

$\equiv$ Hydrocotyle sibthorpioides Lam. var. dichondroides (Makino) M. Hiroe, 1950, Acta Phytotax. Geobot. 14(2): 40.

Type: JAPAN. "Kagoshima, prov. Satsuma, Kyusyu, 08-09. 1909, Makino" (syntype TI); "Sezaki in Ibusuki-gori, 23. 08. 1910, Makino" (syntype TI).

Distribution in China: S: Taiwan.

Distribution outside China: Japan.

Hydrocotyle dielsiana H. Wolff, 1929, Repert. Spec. Nov. Regni Veg. 27: 112.

Type: CHINA. "China, West-Hupeh, Chienshi, 08.1901, Wilson 1592" (lectotype K! - barcode K000686211, designated here); "China, Hubei, Junghu, 1885-1888, Henry 6405B" (syntypes K! barcode K000686212, US).

Distribution in China: Centr.: Hubei; SW: Sichuan. Endemic.

Hydrocotyle handelii $\mathrm{H}$. Wolff ex Hand.-Mazz., 1933, Symb. Sin. 7(3): 707.

$\equiv$ Hydrocotyle burmanica Kurz subsp. handelii (H. Wolff ex Hand.-Mazz.) Wu Zhengyi et Pu Fating, 1993, in Wang Wentsai (ed.), Vasc. Pl. Hengduan Mount.: 1277.

$\equiv$ Hydrocotyle hookeri (C. B. Clarke) Craib subsp. handelii (H. Wolff) M. F. Watson et Sheh Menglan, 2004, Acta Phytotax. Sin. 42(6): 563.

Type: CHINA. "Prov. Yunnan: prope vicum Hsinlung trans flumen Pudu-ho ad septentr. urbis Yunnanfu, $25^{\circ} 34^{\prime}$ lat., in regione calede temperatae, ca. 2350 m, 01. 10. 1914, Handel-Mazzetti 5694" (holotype WU! - barcode WU0060770).
Distribution in China: SW: Sichuan, Guizhou, Yunnan. Endemic.

Hydrocotyle himalaica P. K. Mukh., 1969, Indian Forester 95(7): 470, tab. 1.

$\equiv$ Hydrocotyle javanica Thunb. var. podantha $\mathrm{C}$. B. Clarke, 1879, in Hooker f. (ed.), Fl. Brit. India 2: 668 , non H. podantha Molk.

Type: INDIA. "Khursiong, 1645 m, 24. 09. 1884, Clarke 35825A" (holotype CAL; isotype LE!).

- Hydrocotyle podantha auct. non Molk.: H. Hara, 1966, Fl. East Himal.: 230.

Distribution in China: Tibet: Xizang A. R.; SW: Sichuan, Guizhou, Yunnan; S: Taiwan, Guangdong, Hainan Dao.

Distribution outside China: Myanmar, Bhutan, Nepal, India.

Hydrocotyle hookeri (C. B. Clarke) Craib, 1911, Bull. Misc. Inform. Kew 1911: 59.

$\equiv$ Hydrocotyle javanica Thunb. var. hookeri C.

B. Clarke, 1879, in Hooker f., Fl. Brit. India 2: 668.

Type: INDIA. "Khasia, 5000-6000 ft, 01. 08. 1850, Hooker et Thomson" (lectotype K!, designated here; isolectotypes CAL, MANCH!).

$=$ Hydrocotyle siamica Craib, 1911, Bull. Misc. Inform. Kew 1911: 58.

Type: THAILAND. "Chiengmai, Doi Sootep, 1200-1650 m, 06.06.1909, Kerr 670" (lectotype K!barcode K000686194, designated here; isolectotype BM! - barcode BM000944685); "Chiengmai, Doi Sootep, 13. 12. 1904. Hosseus 192" (syntypes E! barcode E00000016), M - barcode M0168575, P barcode P00757659).

= Hydrocotyle siamensis H. Wolff, 1921, Repert. Spec. Nov. Regni Veg. 17: 155.

Type: THAILAND. "Chiengmai, Gipgel des Doi Sutep, 1700 m. u. M, 17. 04. 1904, Hosseus 192" (holotype ?; isotype E!).

= Hydrocotyle forrestii $\mathrm{H}$. Wolff, 1929, Repert. Spec. Nov. Regni Veg. 27: 113.

$=$ Hydrocotyle burmanica Kurz subsp. forrestii (H. Wolff) Wu Zhengyi et Pu Fating, 1993, in Wang Wentsai (ed.), Vasc. Pl. Hengduan Mount.: 1277.

Type: CHINA. "Mid. West-Yunnan, ShmeliSalwin divide, $25^{\circ} 30^{\prime} \mathrm{N}, 90^{\circ} 38^{\prime}$ E, in shady forests, 1924-1925, Forrest 24834" (lectotype E!, designated by Watson in E; isolectotypes BM! - barcode BM000798551, NY - barcode NY00405996, P barcode P00757653).

- Hydrocotyle burmanica auct. non Kurz: Shan Renhwa et Liou Shoulu, 1964, Acta Phytotax. Sin. 9(2): 128. 
Distribution in China: Tibet: Xizang A. R.; Centr.: Hunan; SW: Sichuan, Yunnan; S: Guangdong.

Distribution outside China: Laos, Thailand, Myanmar, India.

Hydrocotyle maritima Honda, 1934, Bot. \& Zool. (Tokyo) 2: 1825.

$\equiv$ Hydrocotyle ramiflora Maxim. var. maritima (Honda) M. Hiroe, 1950, Acta Phytotax. Geobot. 14(2): 40.

Type: JAPAN. "Honshu, ins. Oshima, prov. Kii, 27. 12. 1933, Jotani" (holotype TI!).

Distribution in China: SW: Yunnan.

Distribution outside China: Japan, Korea.

Hydrocotyle nepalensis Hook., 1822, Exot. F1. 1(2): tab. 30.

$\equiv$ Hydrocotyle hispida Buch.-Ham., 1825, Prodr.

F1. Nepal.: 182, nom. illeg. (Art. 52.1).

$\equiv$ Hydrocotyle nepalensis Hook. var. longipes DC., 1830, Prodr. 4: 65.

Type: NEPAL. "Nepal, 1821. Wallich [561]" (lectotype K! - barcode K001111284, designated by Matthew in $\mathrm{K}$; isolectotypes $\mathrm{BM}, \mathrm{E}$ ! barcode E00041205, K-WALLICH! - barcodes K000686200, K001111283, K001111286, M, P barcode P00757656).

= Hydrocotyle zeylanica DC., 1830, Prodr. 4: 67.

Type: SRI-LANKA. "In Zeylonae circa Kandy locis fertilibus, 1823, Leschenault" (holotype G-DC!).

$=$ Hydrocotyle nepalensis Hook. var. brevipes DC., 1830, Prodr. 4: 65.

Type: not indicated.

= Hydrocotyle polycephala Wight et Arn., 1834, Prodr. Fl. Penins. Ind. Orient. 1: 366.

$=$ Hydrocotyle javanica Thunb. var. polycephala (Wight et Arn.) Masam., 1932, J. Soc. Trop. Agric. Taiwan 4: 300.

Type: INDIA. "Peninsular India Orientalis, Wight 1187" (lectotype K!, designated here; isolectotypes E!, NY, P).

= Hydrocotyle laxiflora Masam., 1932, J. Soc. Trop. Agric. Taiwan 4: 300, nom. illeg., non DC. (1830).

= Hydrocotyle masamunei M. Hiroe, 1979, Umbelliferae World: 156.

Type: CHINA. "[Taiwan] Taiheizan, 07. 08. 1928, Suzuki" (holotype TAI).

= Hydrocotyle javanica Thunb. var. laxa Masam., 1932, J. Soc. Trop. Agric. Taiwan 4: 300.

Type: CHINA. "Isl. Yaku, coll. unclear" (holotype TAI?).
Distribution in China: NW: Gansu; Tibet: Xizang A. R.; N: Shaanxi; Centr.: Henan, Anhui, Hubei, Hunan, Jiangxi, Zhejiang, Fujiang; SW: Sichuan, Guizhou, Yunnan; S: Taiwan, Guangxi Zhuang A.R., Guangdong, Hainan Dao.

Distribution outside China: Japan, Korea, Vietnam, Myanmar, Bhutan, Nepal, India, SriLanka.

Hydrocotyle peltiformis Li Rong et Li Heng, 2013, J. Syst. Evol. 51(2): 223.

Type: CHINA. "Yunnan: Baoshan Prefecture, Longyang, Margkuang township, Baihualing village, spring pool, east side of Gaoligong Shan, second evergreen broad-leaved forest, growing along trail, $28^{\circ} 18^{\prime} 20.9^{\prime \prime} \mathrm{N}, 98^{\circ} 47^{\prime} 59.7^{\prime \prime}$ E, alt. 1600 m, 12. 10. 2007, Li, Li et Shi 1337" (holotype KUN).

Distribution in China: SW: Yunnan. Endemic.

Hydrocotyle pseudoconferta Masam., 1932, J. Soc. Trop. Agric. Taiwan 4: 301.

Type: CHINA. "[Taiwan] Taiheizan, 19. 08. 1925, Suzuki" (syntype TAI); "[Taiwan] Inter Ugan et Pianananbu, 03. 07. 1930, Suzuki" (syntype TAI).

- Hydrocotyle conferta auct. non Wight: Matsum. \& Hayata, 1906, Enum. Pl. Formosan.: 170.

- Hydrocotyle nepalensis auct. non Hook.: Liu Tangshui \& Kao Muhtsien, in Li Huilin et al. (eds.), Fl. Taiwan 3: 956. 1977, p.p.

Distribution in China: Centr.: Jiangsu, Zhejiang; SW: Yunnan; S: Taiwan.

Distribution outside China: Japan, Philippines, Myanmar.

Hydrocotyle ramiflora Maxim., 1886, Bull. Acad. Imp. Sci. Saint-Petersbourg 31: 46.

Type: JAPAN. "Japonia, circa Hakodate, in humidis vulgaris, 27. 06. 1861, Maximowicz" (lectotype LE!, designated here; isolectotypes BM! barcode BM0000944687, M - barcode M0172688, $\mathrm{P}$ - barcode P00757662).

Distribution in China: Centr.: Jiangxi, Zhejiang, Fujiang; SW: Sichuan, Guizhou, Yunnan; S: Taiwan, Guangxi Zhuang A. R., Guangdong, Hainan Dao.

Distribution outside China: Russia, Japan, Korea, Vietnam, India, Georgia, Turkey.

Hydrocotyle salwinica Shan Renhwa et Liou Shoulu, 1964, Acta Phytotax. Sin. 9(2): 131, tab. 8, fig. $1-5$.

$\equiv$ Hydrocotyle himalaica P.K.Mukh subsp. salwinica (Shan Renhwa et Liou Shoulu) Wu Zhengyi et Pu Fating, 1993, in Wang Wentsai (ed.), Vasc. P1. Hengduan Mount.: 1278. 
Type: CHINA. "Yunnan, Salwin valley, SW of Champutong, alt. $1850 \mathrm{~m}$., waste land, 24. 06. 1938, Yu Tsetsun 19142" (holotype E! - barcode E00000018; isotypes A, KUN!, NAS!, PE!).

Distribution in China: Tibet: Xizang A. R.; SW: Yunnan. Endemic.

Hydrocotyle salwinica Shan Renhwa et Liou Shoulu var. obtusiloba Liou Shoulu, 1990, Acta Phytotax. Sin. 28(2): 152.

Type: CHINA. "Xizang: Mailing, 2500-3100 m alt., 26. 07. 1972, Xizang Exped. Plant. Officin. 4181" (holotype PE).

Distribution in China: Tibet: Xizang A. R. Endemic.

Hydrocotyle setulosa Hayata, 1908, J. Coll. Sci. Imp. Univ. Tokyo 25(19): 102.

Type: CHINA. "[Taiwan] Arisan, in montibus Morrison, 09. 11. 1906, Nakahara" (holotype TI!).

Distribution in China: S: Taiwan. Endemic.

Hydrocotyle sibthorpioides Lam., 1789, in Lamarck et al. (eds.), Encycl. 3(1): 153.

$\equiv$ Chondrocarpus sibthorpioides (Lam.) Sweet, 1826, Hort. Brit. 1: 185.

Type: MAURITIUS. "Île de France [Mauritius], Sonnerat, Commerson (Herb. Lamarck)" (syntypes P - barcode P00312676, MPU - barcodes MPU019295, MPU020467); "Mauritius, Du PetitThouars" (syntype P).

$=$ Hydrocotyle nitidula Rich., 1820, Ann. Gen. Sci. Phys. 4: 200, tab. 63, fig. 33.

Type: INDONESIA. "L'île de Java".

= Hydrocotyle tenella D. Don, 1825, Prodr. F1. Nepal.: 183.

Type: NEPAL. "In Nepalia, Buchanan-Hamilton [562.2]" (lectotype K-WALLICH!, designated here; isolectotype BM!).

= Hydrocotyle splendens Blume, 1826, Bijdr. fl. Ned. Ind. 15: 884.

Type: INDONESIA. "In graminosis humidis circa Buitenzorg" (holotype L?).

$=$ Hydrocotyle ranunculoides L.f. var. incisa Blume, 1826, Bijdr. fl. Ned. Ind. 15: 884.

Type: INDONESIA. "In graminosis humidis circa Buitenzorg" (holotype L?).

= Hydrocotyle rotundifolia Roxb. ex DC., 1830, Prodr. 4: 64.

Type: INDIA/BANGLADESH. "In Sylhet Bengali orientalis, Wallich [562]" (lectotype G-DC!, designated here; isolectotypes K! barcode K001111287, K-WALLICH! - barcodes
K001111288, K001111289, LE! - barcode LE00015623).

= Hydrocotyle hirsuta Blume, 1826, Bijdr. Fl. Ned. Ind. 15: 884. 1826; et ex DC., 1830, Prodr. 4: 67, nom. illeg. (Art. 53.1), non Swartz (1788).

Type: INDONESIA. "In graminosis maritimis Bataviae" (holotype L?).

= Hydrocotyle zollingeri Molk., 1851, P1. Jungh. 1: 91.

Type: INDONESIA. "Hab. Javam insulam, Zollinger 834" (lectotype K! - barcode K000686197, designated here; isolectotypes K! - barcode K000686196; LE!); "In Sumatrae Provincia Padang-Lawa's, Junghuhn" (syntype); "In sylvis intactis Javanicis provinciae Medini ad latus septentr. montis Ungaran, alt. 3-5000', Junghuhn" (syntype).

= Hydrocotyle perexigua Hance, in Walpers, 1852, Ann. Bot. Syst. 2: 691.

Type: CHINA. "In insula Hongkong locis humidis subumbrosis, Hance 564" (lectotype $\mathrm{K}$ !, designated here; isolectotype $\mathrm{BM}$ ! - barcode BM000798553).

= Hydrocotyle latisecta Zoll., 1854, Syst. Verz. 2: $139,140$.

Type: INDONESIA. "Samangi. In graminosis, ad vias prope Gebok klakka. Pr. Passaruan, 2-4000', H.2512".

= Hydrocotyle puncticulata Miq., 1855, Fl. Ned. Ind. 1(1): 732.

Type: INDONESIA. "Java, in Soerakarta door Horsfield ontdekt [Java], Horstfield 1109" (type K!).

Distribution in China: Tibet: Xizang A. R.; N: Shaanxi, Shandong; Centr.: Henan, Jiangsu, Anhui, Hubei, Hunan, Jiangxi, Zhejiang, Fujiang; SW: Sichuan, Guizhou, Yunnan; S: Taiwan, Guangxi Zhuang A. R., Guangdong, Hainan Dao.

Distribution outside China: Japan, Korea, Philippines, Papua-New Guinea, Indonesia, Malaysia, Vietnam, Thailand, Myanmar, Bangladesh, Bhutan, Nepal, India, Sri-Lanka, Pakistan, Georgia, Yemen, Syria, Israel. Europe, Africa, N. America, Australia and Oceania.

Hydrocotyle tuberifera Ohwi, 1951, J. Jap. Bot. 26: 233.

$\equiv$ Hydrocotyle sibthorpioides Lam. var. tuberifera (Ohwi) T. Yamaz., 1994, J. Jap. Bot. 69(2): 112.

Type: JAPAN. "Liukiu. Ins. Okinawa, Shuri, 26. 07. 1894, Ito 792" (lectotype TNS!, designated here; isolectotype TI!).

$=$ Hydrocotyle keelungensis Liu Tangshui, Chao Chuangying et Chuang Tsaniang, 1961, Quart. J. Taiwan Mus. 14(1-2): 29. 
Type: CHINA. "Taiwan, Keelung, Chuang 3980" (holotype TAI).

Distribution in China: S: Taiwan,

Distribution outside China: Japan.

Hydrocotyle wilfordii Maxim., 1887, Bull. Acad. Imp. Sci. Saint-Petersburg 31: 45.

Type: JAPAN. "Japonia, circa Hakodate, 26. 06. 1861, Maximowicz" (syntype LE!); "Nippon, circa Yokohama, Maximowicz" (syntype LE?). KOREA. "Corea, Portu Chusan, 1859, Wilford" (lectotype LE!, designated by Grubov (Vinogradova, 2004: 29); isolectotype GH).

Distribution in China: Tibet: Xizang A. R.; Centr.: Hunan, Hubei, Jiangxi, Zhejiang, Fujiang; SW: Sichuan, Guizhou, Yunnan; S: Taiwan, Guangxi Zhuang A.R., Guangdong, Hainan Dao.

Distribution outside China: Japan, Korea, Vietnam.

Hydrocotyle wilsonii Diels ex Shan Renhwa et Liou Shoulu, 1964, Acta Phytotax. Sin. 9(2): 128.

Type: CHINA. "Hupeh: Patung district, Henry 4725" (lectotype K!, designated here); "W China, Changyang, Wilson 1465" (syntype K!).

Distribution in China: Centr.: Hubei. Endemic.

Hymenidium album (C. B. Clarke ex H. Wolff) Pimenov et Kljuykov, 2000, Feddes Repert. 111(78): 549.

$\equiv$ Pleurospermum album C. B. Clarke ex H. Wolff, 1929, Repert. Spec. Nov. Regni Veg. 27: 113.

Type: CHINA. "Flora of Chumbi et Parhi, IoKir-La , 07. 1879, Dungboo" (holotype K! - barcode K000685302; photo E).

Distribution in China: Tibet: Xizang A. R.

Distribution outside China: Bhutan, Nepal, India.

Hymenidium amabile (Craib et W. W. Sm.) Pimenov et Kljuykov, 2000, Feddes Repert. 111(78): 545.

$\equiv$ Pleurospermum amabile Craib et W. W. Sm., 1913, Trans. Bot. Soc. Edinburgh 26: 154.

Type: CHINA. "East Himalaya. At Chulong in the Chumbi valley, Tibet, at an elevation of 15000 feet, 08. 1912, Rohmoo Lepcha 207" (lectotype E! barcode E00000206, designated here; isolectotypes CAL, E, K! - barcode K000685343).

Distribution in China: Tibet: Xizang A. R.; SW: Sichuan, Yunnan.

Distribution outside China: Bhutan, India.
Hymenidium apiolens (C. B. Clarke) Pimenov et Kljuykov, 2000, Feddes Repert. 111(7-8): 546.

$\equiv$ Pleurospermum apiolens C. B. Clarke, 1879, in Hooker f. (ed.), Fl. Brit. India 2: 705.

Type: INDIA. "Sikkim, Regio Alp., alt. 1100014000 ped [Tunju Ortoba, Tongsi], Hooker" (lectotype K! - barcode K000685319, designated by Mukherjee, Constance, 1993: 191; isolectotypes BM!, G! - barcode G00367224, MH!, NY barcode NY00406200, P! - barcodes P008344572, P008344573, P008344574, W!); "Sikkim, Regio temp., Yeumting, 05-12.09.1849, Hooker" (syntypes K - barcodes K000685323, K000685324); "Sikkim, Lachuang, 30. 08. 1849, Hooker" (syntype K barcode K000685322).

= Pleurospermum atropurpureum $\mathrm{Fu}$ Kuntsun et Ho Yehchi, in Shan Renhwa \& Sheh Menglan, 1979, Fl. Reipubl. Popularis Sin. 55(1): 298,161, tab. 82, fig. 1-9.

Type: CHINA. "Tibet, Nelamu Hsien, alt. 3800 m, 02. 09. 1972, Tibetan medicinal plant collection 1723 ".

= Pleurospermum apiolens C. B. Clarke var. nipaulensis Farille et Malla, 1985, Candollea 40(2): 524, fig. 9 (3-5).

Type: NEPAL. "E Nepal: Magar ko Danda-la, $3500 \mathrm{~m}$. Lieux herbeux extrêmement pentueux, 17. 10. 1981, Farille 81-697" (holotype KATH; isotypes E! - barcode E00000208, G! - barcode G00367226).

Distribution in China: Tibet: Xizang A. R.

Distribution outside China: Bhutan, Nepal, India.

Hymenidium astrantioideum (H. Boissieu) Pimenov et Kljuykov, 2000, Feddes Repert. 111(78): 545

$\equiv$ Trachydium astrantioideum H. Boissieu, 1906, Bull. Soc. Bot. France 53: 422.

$\equiv$ Pleurospermum astrantioideum (H. Boissieu) Fu Kuntsun et Ho Yehchi, 1979, in Shan Renhwa \& Sheh Menglan (eds.), Fl. Reipubl. Popularis Sin. 55(1): 178, tab. 94, fig. 1-3.

Type: CHINA. "Thibet oriental: Province de Batang, Yargong, eboulis des terrains sablonneux des hautes montagnes, 07. 1903, Soulié 3204" (lectotype P! - barcode P00834662, designated here; isolectotype $\mathrm{P}$ - barcode P00245433).

= Pleurospermum thalictrifolium $\mathrm{H}$. Wolff, 1926, Acta Horti Gothob. 2: 297.

Type: CHINA. "Sze-ch'uan bor.: Dongrergo, auf Schutthalden, 4700 m, 09. 08. 1922, H. Smith 3151" (syntype UPS!); “Sze-ch'uan, reg. bor.: Dongrergo, 
4900-5000 m, 09. 08. 1922, H. Smith 3972" (syntypes GB! - barcode GB-0048825, UPS!); "Sze-ch'uan, reg. bor.: Dongrergo, in declivitate microlapidosa, Huang lung ssu, 4700-4800 m, 11. 08. 1922, H. Smith 3233" (lectotype GB! - barcode GB-0048824, designated by Pimenov, Kljuykov, 2000: 545; isolectotypes UPS!, W!).

Distribution in China: Tibet: Xizang A. R.; SW: Sichuan. Endemic.

Hymenidium bicolor (Franch.) Pimenov et Kljuykov, 2000, Feddes Repert. 111(7-8): 545.

$\equiv$ Pleurospermum govanianum (DC.) Benth. ex C. B. Clarke var. bicolor Franch., 1894, Bull. Annuel Soc. Philom. Paris (sér. 8) 6: $137 .$.

$\equiv$ Pleurospermum bicolor (Franch.) C. Norman ex Pan Zehui et M. F. Watson, 2004, Acta Phytotax. Sin. 42(6): 564.

Type: CHINA. "[Yunnan] Mt. Tsangchan, supra Tali, alt. 4000 m, 29. 09. 1884, Delavay 193" (lectotype P! - barcodes P00834599, P00834600, designated by Pimenov, Kljuykov, 2000: 545; isolectotypes K!, P - barcode P00834598); "[Yunnan] Ma-eul-chan. Alt. 3500 m, 23. 10. 1889, Delavay" (syntypes K! - barcode K000685341, P! barcode P00834601); "Yunnan, in monte Koua-lapo, in pratis, alt. $3200 \mathrm{~m}, 26.08 .1884$, Delavay 949” (syntype P! - barcodes P00834593, P00834594, P00834595, P00834597).

= Pleurospermum govanianum (DC.) Benth. ex C. B. Clarke var. bicolor H. Wolff, 1929, Repert. Spec. Nov. Regni Veg. 27: 115.

Type: CHINA. "Eastern slopes of Likiang Snow range, Yangtze watershed, 19. 09. 1923, Rock 10781" (holotype UC!; isotype US!).

Distribution in China: Tibet: Xizang A. R.; SW: Sichuan, Yunnan. Endemic.

Hymenidium chloroleucum (Diels) Pimenov et Kljuykov, 2000, Feddes Repert. 111(7-8): 548.

$\equiv$ Trachydium chloroleucum Diels, 1912, Notes Roy. Bot. Gard. Edinburgh 5: 290.

Type: CHINA. "Grassy openings in pine forests on the eastern flank of the Lichiang Range. Lat. $27^{\circ} 25^{\text {' }}$ N. Alt. 11000-12000 ft., 09. 1906, Forrest 2886" (lectotype E! - barcode E00000280, designated by Pimenov, Kljuykov, 2000: 548; isolectotypes BM! barcode BM000559385, P! - barcode P00834540); "Stony, mountain pastureland on the eastern flank of the Lichiang Range. Lat. $27^{\circ} 20^{\prime}$ N. Alt. 11-12000 ft., 08. 1906, Forrest 2873" (syntypes BM - barcode BM000944689, E! - barcode E00000279, P).

= Pleurospermum likiangense H. Wolff, 1930, Repert. Spec. Nov. Regni Veg. 27: 321, nom. inval.
= Pleurospermum yulungense $\mathrm{Wu}$ Zhengyi, 1984, Index Fl. Yunnan. 1: 923.

Type: CHINA. "Yangtze watershed, prefectural district of Likiang, eastern slopes of Likiang Snow Range, Rock 9854" (syntypes E! - barcode E00041466, P! - barcode P00245449, UC!); "Yangtze watershed, prefectiral district of Likiang, eastern slopes of Likiang Snow Range, Rock 5261" (lectotype US!, designated by Pimenov, Kljuykov, 2000: 548; isolectotype W!).

= Ligusticum sikiangense M. Hiroe, 1958, Umbell. Asia 1: 107, "sikiangensis".

Type: CHINA. "Prov. Sikang: under wood, alt. 3650 m, Ching-Chang, Wachin, Mu-li, 21. 06. 1937, T. T. Yu 6538" (holotype UC! - barcode UC792285; isotype PE!).

Distribution in China: Tibet: Qinghai; SW: Sichuan, Yunnan. Endemic.

Hymenidium cristatum (H. Boissieu) Pimenov et Kljuykov, 2000, Feddes Repert. 111(7-8): 542.

$\equiv$ Pleurospermum cristatum H. Boissieu, 1906, Bull. Soc. Bot. France 53: 434.

Type: CHINA. "Prov. Hupeh, Ichang, Hsing shan, Henry 6510" (lectotype K! - barcode K000685350, designated here; isolectotype P! barcode P00834577).

Distribution in China: NW: Gansu; Tibet: Qinghai; N: Ningxia Huizu A.R., Shaanxi, Shanxi; Centr.: Henan, Anhui, Hubei; SW: Sichuan. Endemic.

Hymenidium davidii (Franch.) Pimenov et Kljuykov, 2000, Feddes Repert. 111(7-8): 542.

$\equiv$ Pleurospermum davidii Franch., 1885, Nuov. Arch. Mus. Hist. Nat. (sér. 2) 8 (Pl. David. 2): 247, tab. 8, fig. B.

Type: CHINA. "Thibet oriental, Moupine, haute prairie à plus de $4000 \mathrm{~m}, 08.1869$, David" (lectotype P! - barcode P00834575, designated here; isolectotype $\mathrm{P}$ - barcode $\mathrm{P} 00834576$ ).

= Pleurospermum franchetianum Hems1., 1892, J. Linn. Soc. Bot. 29: 308.

Type: CHINA. "West Szechuen and Tibetan frontier, chiefly near Tachienlu, at 9000-15000 ft. [Ta-Tsien-Lou (Setchuen)], Pratt 552" (lectotype P! - barcode P00834592, designated here; isolectotypes BM! - barcode BM000944694, K! barcode K000685348).

= Pleurospermum grandifolium H. Wolff, 1925, Repert. Spec. Nov. Regni Veg. 21: 244..

Type: CHINA. "Tibetia orientalis: Ta-tsien-lu, 08. 1894, Soulié 2182[2183?]" (holotype P?). 
= Pleurospermum tanacetifolium $\mathrm{H}$. Wolff, 1926, Acta Horti Gothob. 2: 293.

Type: CHINA. "Sze-ch'uan, reg. bor:: O von Sung-pan, bustliang. $3300 \mathrm{~m}, 13.07 .1922$, H. Smith 2942" (lectotype GB! - barcode GB0048823, designated by Pimenov, Kljuykov, 2000: 543; isolectotypes UPS!, W!); 'Sze-ch'uan bor: Hsioeh-shan, in prato alpino, 3900-4200 m, 24. 07. 1922, H. Smith 3694" (syntype UPS!); "Sze-ch'uan bor.: San-ch'a-tzu, in prato, $4000 \mathrm{~m}, 10.08 .1922$, H. Smith 3412" (syntype UPS!).

= Pleurospermum pilgerianum Fedde ex $\mathrm{H}$. Wolff, 1929, Repert. Spec. Nov. Regni Veg. 27: 121.

Type: CHINA. "SW-Kansu: Tao River basin, below Mt. Lissedzadza, on conglomerate cliffs, in crevices, $11500 \mathrm{ft}$., 07. 07. 1925, Rock 12614"(lectotype GH - barcode GH00062616, designated here; isolectotype BM! - barcode BN000944692).

= Pleurospermum rockii Fedde ex H. Wolff, 1929, Repert. Spec. Nov. Regni Veg. 27: 120.

Type: CHINA. "SW-Kansu: Tao river basin, banks of Tao river, below Choni, grassy slopes, 8300 ft.,06. 1925, Rock 12227" (lectotype BM! - barcode BM000944693, designated here; isolectotypes E!, K - barcode K000685347, GH - barcode GH00062615, US - barcode US00730805).

- Pleurospermum benthamii auct. non C. B. Clarke: Pan Zehui et M. F. Watson, 2005, in Wu Zhengyi \& Raven (eds.), Fl. China 14: 46.

Distribution in China: NW: Gansu; Tibet: Xizang A. R., Qinghai; N: Ningxia Huizu A.R., Shaanxi; Centr.: Henan, Hubei; SW: Sichuan, Yunnan. Endemic.

Hymenidium decurrens (Franch.) Pimenov et Kljuykov, 2000, Feddes Repert. 111(7-8): 542.

$\equiv$ Pleurospermum decurrens Franch., 1894, Bull. Annuel Soc. Philom. Paris (sér. 8) 6: 138.

Type: CHINA. "Province du Yun-nan, Likiang Suee chan, in pratis, alt. $4000 \mathrm{~m}, 14.08 .1886$, Delavay" (syntype P! - barcode P00834586); "Province du Yun-nan, les prairies de Tsang chan au-dessus de Ta-li, 4. 07. 1882, Delavay 451" (lectotype P! - barcode P00834578, designated by Pimenov, Kljuykov, 2000: 542; isolectotype P barcode P00834579); "Province du Yun-nan, parmi les brouillards au pied du Tsang chan, 30. 08. 1889, Delavay" (syntypes K!, P!); "Province du Yun-nan, parmi les brouillards du Tsang chan, au dessus de Ta-li, 26. 09. 1884, Delavay 674" (syntypes K barcode K000685349, P! - barcodes P00834582, P00834583, P00834584).
Distribution in China: Tibet: Xizang A. R.; SW: Sichuan, Yunnan. Endemic.

Hymenidium delavayi (Franch.) Pimenov et Kljuykov, 1999, Feddes Repert. 110(7-8): 484, fig. $1, \mathrm{C}, \mathrm{D}$.

$\equiv$ Ligusticum delavayi Franch., 1894, Bull. Annuel Soc. Philom. Paris (sér. 8) 6: 131.

Type: CHINA. "Au côte de Yen-tze-hay (Lankong), $3000 \mathrm{~m}$ d'altid., 18. 09. 1885, Delavay 675" (lectotype P! - barcode P03224808, designated by Leute, 1970: 491; isolectotypes B!, K!, P - barcodes $\mathrm{P} 03224571, \mathrm{P} 03224584, \mathrm{P} 03224585, \mathrm{P} 03224812$, P03224813); "Yun-nan, in pratis ad collum Yentze-hay, prope lacum, alt. $3200 \mathrm{~m}, 19.07 .1887$, Delavay 3087" (syntype K! - barcode K000695863, P! - barcode P03224572); "Yun-nan, in humidis ad collum Hec chan men, 28. 10. 1889, Delavay 4221" (syntype P! - barcodes P03224809, P03225473).

$=$ Pleurospermum meoides Diels, 1900, Bot. Jahrb. Syst. 29 (3-4) (Fl. Centr. China): 493.

Type: CHINA. "Setchuen, Nan Ch'uan: Hei wan Ch`i, 25. 09. 1891, von Rosthorn 1013" (holotype $\mathrm{O}$ !).

Distribution in China: Tibet: Xizang A. R.; SW: Sichuan, Yunnan. Endemic.

Hymenidium dentatum (DC.) Pimenov et Kljuykov, 2000, Feddes Repert. 111(7-8): 546.

$\equiv$ Hymenolaena dentata DC., 1830, Prodr. 4: 245.

$\equiv$ Pleurospermum dentatum (DC.) Benth. ex C. B. Clarke, 1879, in Hooker f. (ed.), Fl. Brit. India 2: 704 .

Type: NEPAL. "Gossain Than. 1821, Wallich [547]" (lectotype G-DC!, designated by Pimenov, Kljuykov, 2000: 546; isolectotypes BM!, CAL, E, K!, K-WALLICH!, LE!).

= Hymenolaena pumila DC., 1830, Prodr. 4: 245.

= Pleurospermum pumilum (DC.) Benth. ex C.

B. Clarke, 1879, in Hooker f. (ed.), Fl. Brit. India 2: 704 .

Type: NEPAL. "Ad Gossain-Than Nepalensium, Wallich [550]" (lectotype G-DC!, designated by Pimenov, Kljuykov, 2000: 547; isolectotypes K!, K-WALLICH!).

= Pleurospermum dentatum (DC.) Benth. ex C. B. Clarke var. erosum (DC.) C. B. Clarke, 1879, in Hooker f. (ed.), Fl. Brit. India 2: 704.

Type: INDIA/NEPAL. "In Nepalia ad Kamaon et Gossain-Than, Wallich" (holotype G-DC).

= Hymenolaena dentata DC. var. erosa DC., 1830, Prodr. 4: 245. 
= Hymenolaena erosa (DC.) Steud., 1840, Nomencl. Bot. (ed. 2) 1: 784.

= Pleurospermum erosum (DC.) P. K. Mukh., 1978, in Cauwet-Marc \& Carbonier (eds.), Ombel. Contr. Pluridisc. Syst.: 61, "erosa", nom. inval. (Art. 41.5), sine basion.

Type: NEPAL. "Napaul, Wallich" (lectotype G-DC!, designated by Pimenov, Kljuykov, 2000: 547 ; isolectotypes $\mathrm{K}$ !, CAL).

Distribution in China: Tibet: Xizang A. R.

Distribution outside China: Bhutan, Nepal, India.

Hymenidium foetens (Franch.) Pimenov et Kljuykov, 2000, Feddes Repert. 111(7-8): 544.

$\equiv$ Pleurospermum foetens Franch., 1894, Bull. Annuel Soc. Philom. Paris (sér. 8) 6: 140.

Type: CHINA. "Yun-nan, Likiang in collibus calcareis prope nives perpetuas, alt. $3800-4000 \mathrm{~m}$ [Côteaux pierreux et calcairs a $3800 \mathrm{~m}$, glacier de Likiang], 11. 07. 1884, Delavay 677" (lectotype P! - barcode P00834590, designated by Pimenov, Kljuykov, 2000: 544; isolectotype P - barcode P00834591); "Province du Yun-nan, côteaux pierreux et calcairs, glacier de Likiang, 09. 07. 1884, Delavay 835" (syntype P! - barcodes P00834588, P00834589).

Distribution in China: NW: Gansu; Tibet: Xizang A. R.; SW: Sichuan, Yunnan. Endemic.

Hymenidium giraldii (Diels) Pimenov et Kljuykov, 2000, Feddes Repert. 111(7-8): 548.

$\equiv$ Pleurospermum giraldii Diels, 1900. Bot. Jahrb. Syst. 29 (3-4) (Fl. Centr. China): 492.

Type: CHINA. "Gipfel des T'ai pa shan [China inferior, provincia Shan-si septentr. M-te Thae pei san], 10-20. 08. 1897, Giraldi 1337" (lectotype FI!, designated here; isolectotype FI!, K! - barcode K000685337).

= Pleurospermum limprichtii H. Wolff, 1922, Repert. Spec. Nov. Regni Veg. Beih. 12 (Limpr. Bot. Reise Hochgeb. China Ost-Tibet): 477.

Type: CHINA. "Schenhsi: Tsin ling schan, Tai pai schan, 3500 m, 01. 08. 1916, Limpricht 2769" (holotype WU!).

Distribution in China: NW: Gansu; N: Shaanxi; Centr.: Hubei; SW: Sichuan. Endemic.

Hymenidium hedinii (Diels) Pimenov et Kljuykov, 2000, Feddes Repert. 111(7-8): 550.

$\equiv$ Pleurospermum hedinii Diels, 1922, in Hedin (ed.), S.Tibet 6(3): 52, tab. 6, fig. 5-6.

$\equiv$ Cortiella hedinii (Diels) C. Norman, 1937, J. Bot. 75: 95.
Type: CHINA. "Tibetia orientalis, prope campus XLIV, 5127 m s.m., 18. 08. 1901, Hedin, Ostenfeld et Paulsen" (syntypes C!, S! - barcodes S-G-4922, S-G-4923); "N Tibet, Camp 26, 4946 m, 30. 06. 1901, Hedin" (lectotype S!, designated here).

Distribution in China: Tibet: Xizang A. R., Qinghai; SW: Sichuan, Yunnan. Endemic.

Hymenidium heracleifolium (Franch. ex H. Boissieu) Pimenov et Kljuykov, 2000, Feddes Repert. 111(7-8): 543.

$\equiv$ Pleurospermum heracleifolium Franch. ex H. Boissieu, 1906, Bull. Soc. Bot. France 53: 433.

Type: CHINA. "Yunnan, 11. 07. 1890, Prince Henri d'Orleans" (holotype P! - barcode P00834539).

Distribution in China: Tibet: Xizang A. R.; SW: Sichuan, Yunnan. Endemic.

Hymenidium heterosciadium ( $\mathrm{H}$. Wolff) Pimenov et Kljuykov, 2000, Feddes Repert. 111(78): 548.

= Pleurospermum heterosciadium H. Wolff, 1925, Repert. Spec. Nov. Regni Veg. 21: 243.

Type: CHINA. "Tibetia orientalis: Tongolo, 07. 1894, Soulié 2611" (holotype P?).

Distribution in China: Tibet: Xizang A. R.; SW: Sichuan, Yunnan. Endemic.

Hymenidium hookeri (C. B. Clarke) Pimenov et Kljuykov, 2000, Feddes Repert. 111(7-8): 547.

$\equiv$ Pleurospermum hookeri C. B. Clarke, 1879, in Hooker f. (ed.), Fl. Brit. India 2: 705.

$\equiv$ Aulacospermum hookeri (C. B. Clarke) Farille et Malla, 1985, Candollea 40(2): 525, fig. 3, 4.

Type: INDIA. "Sikkim, alt. 14000-16000 ft., Hooker" (lectotype K! - barcode K000685316, designated by Constance in $\mathrm{K}$; isolectotypes BM! barcode BM000622355, photo E); "Sikkim, Hooker 08” (syntypes K! - barcode K000685305, P! barcode P00834542, W!).

- Pleurospermum hookeri C. B. Clarke var. thomsonii C. B. Clarke, 1879, in Hooker f. (ed.), Fl. Brit. India 2: 705.

$=$ Aulacospermum hookeri (C. B. Clarke) Farille et Malla var. thomsonii (C. B. Clarke) Karthik et Moorthy, 2009, Fl. Pl. India: 101.

Type: INDIA. "West Tibet, 14-16000 ft., 1213. 09. 1847, Thomson" (lectotype BM! - barcode BM000944725, designated here; isolectotypes G! barcodes G00367228, G00367241, K - barcodes K000685309, K000685311， K000685312); "Shelshel River, 15500 feet, Strachey, Winterbottom 4" (syntypes BM!, K!). 
= Pleurospermum tibetanicum H. Wolff, 1922, Repert. Spec. Nov. Regni Veg. Beih. 12 (Limpr. Bot. Reise China Ost-Tibet): 448.

Type: CHINA. "Ost-Tibet: Batang-Litang, Pung-tschamu, Matten und Geroll des DshagalaPasses, 5000 m, Limpricht 2258" (holotype WU! barcode WU0025955).

= Pleurospermum markgrafianum $\mathrm{H}$. Wolff, 1926, Acta Horti Gothob. 2: 294.

Type: CHINA. "Sze-ch'uan bor.: Hsioeh-shan, auf alpinen Wiesen, 4100-4300 m, 11. 08. 1922, H. Smith 3411" (lectotype UPS!, designated here; isolectotype GB! - barcode GB-0048822).

= Pleurospermum affine H. Wolff, 1926, Acta Horti Gothob. 2: 295.

Type: CHINA. "Sze-chu'an, reg. bor.-occid.: Matang, montes bor--orientales in prato alpino, 4800 m, 15. 09. 1922, H. Smith 4454" (lectotype UPS!, designated here; isolectotypes BM! - barcode BM000944690, GB! - barcode GB-0048820, PE!, $\mathrm{W}$ !).

= Pleurospermum wolffianum Fedde ex H. Wolff, 1929, Repert. Spec. Nov. Regni Veg. 27: 119.

Type: INDIA. "East Himalaya: Kalaeree, 16000 ft., 1912, Rohmoo Lepcha 441" (holotype K!; photo E00265124; isotype E - barcode E00000215).

= Pleurospermum hookeri C. B. Clarke var. haidongense Pan Jintang, 1999, in Fl. Qinghaica 2: 434, 396.

Type: CHINA. "Qinghai: Huangzhong, in pratis alpinis, alt. $3940 \mathrm{~m}, 19.07 .1993$, T. N. Ho, Bartholomew et Gilbert 2" (holotype HNWP).

Distribution in China: Tibet: Xizang A. R., Qinghai; SW: Sichuan, Yunnan.

Distribution outside China: Bhutan, Nepal, India, Pakistan, Afghanistan.

Hymenidium huzhihaoi Pimenov et Kljuykov, 2004, Bot. Zhurn. (St. Petersburg) 89(10): 1659, fig. 6,7 .

Type: CHINA. "Sichuan, Kanding Co., near Tongolo (Dongolou). Lat. 3003' N. Long. 101²9' E. Alt. 3700 m, 21. 09. 1998, Pimenov, Kljuykov, Hu Zhihao et Liu Qixing 304" (holotype MW!; isotype LE!).

Distribution in China: SW: Sichuan. Endemic.

Hymenidium kansuense (H. Wolff) Pimenov et Kljuykov, 2000, Feddes Repert. 111(7-8): 547.

$\equiv$ Pleurospermum kansuense H. Wolff, 1929, Repert. Spec. Nov. Regni Veg. 27: 115.

Type: CHINA. "Kansu: Dar-Pan, near Sining, alt. 3000-3350 m, 26. 07. 1923, R. C. Ching 650" (lectotype UC!, designated here; isolectotype US).
= Pleurospermum pseudoinvolucratum $\mathrm{H}$. Wolff, 1929, Repert. Spec. Nov. Regni Veg. 27: 119.

Type: CHINA. "SW-Kansu: Upper Tebbu country, southern slopes of Minshan, foot of limestone cliffs, 10600 ft., 06. 1925, Rock 12496" (holotype GH; isotype BM! - barcode BM000944691).

Distribution in China: China (NW: Gansu; Tibet: Qinghai).

Distribution outside China: Endemic to Central Asia.

Hymenidium ladyginii Pimenov et Kljuykov, 2011, Bot. Zhurn. (St. Petersburg) 96(5): 648.

Type: CHINA. "Qinghai, jugum Kukunor australi [Qinghai Nanshan], declive australe, amniculus Usunbin-gol, in rupis fluminis argillosis et argilloso-arenosis, $\mathrm{h}=10500$ pes, 16. 08. 1901, Ladygin 434" (holotype LE!).

Distribution in China: Tibet: Qinghai. Endemic.

Hymenidium Ihasanum Pimenov et Kljuykov, 2004, Bot. Zhurn. (St. Petersburg) 89(10): 1657, fig. $4,5$.

Type: CHINA. " Xizang, SE Tibet, Nyainqentanglha Shan. Yangbajain - Damxung, NW of Lhasa. Valley S of Nyainqentanglha Feng. N 30²0', E 90³4'. 5250 m. Among large granite boulders, alpine, 13. 08. 1989, Dickore 3917" (holotype MW!; isotype GOET!).

Distribution in China: Tibet: Xizang A. R. Endemic.

Hymenidium linearilobum (W. W. Sm.) Pimenov et Kljuykov, 2000, Feddes Repert. 111(7-8): 543.

$\equiv$ Pleurospermum linearilobum W. W. Sm., 1915, Notes Roy. Bot. Gard. Edinburgh 8: 342.

Type: CHINA. "Yunnan, West China. Eastern flank of the Lichang Range. Lat. $2720^{\prime}$ N. Alt. 10000-11000 ft. Shady situations amongst scrub, 07. 1910, Forrest 6181"(lectotype E! - barcode E00000146, designated by Pimenov, Kljuykov, 2000: 543; isolectotypes K - barcode K000685346, US!); "Yunnan. Shady openings in pine and mixed forests on the eastern flank of the Likiang Range, Lat. $27^{\circ} 20^{\prime}$ N. Alt. 10000-11000 ft., 07. 1910, Forrest 6051" (syntypes BM! - barcode BM 000944729, E! barcode E00000147).

= Pleurospermum calcareum H. Wolff, 1929, Repert. Spec. Nov. Regni Veg. 27: 114.

Type: CHINA. "Yunnan: prefectural district of Likiang, Yangtze watershed, eastern slopes of Likiang Snow Range, 9500 ft., 27. 06. 1922, Rock 4722" (holotype US). 
= Pleurospermum dielsianum Fedde ex H. Wolff, 1929, Repert. Spec. Nov. Regni Veg. 27: 121.

Type: CHINA. "Eastern Tibet: grasslands between Labrang and Yellow River, on gravelly eastern slopes of Wago (Waro) Kandom Pap, 13000 ft., 25. 07. 1926, Rock 14466" (syntypes BM! - barcode BM000559386, GH - barcode GH00062641, US); "Eastern Tibet: Radja and Yellow River gorges: meadows of mountain southwest of Radja. Alt. 12000 ft., 06.1926, Rock 14178" (lectotype US!, designated by Pimenov, Kljuykov, 2000: 544; isolectotypes BM!, E!, K barcode K000685345).

Distribution in China: Tibet: Qinghai; SW: Sichuan, Yunnan. Endemic.

Distribution outside China: Endemic to Eastern Asia.

Hymenidium macrochlaenum (Fu Kuntsun et Ho Yehchi) Pimenov et Kljuykov, 2000, Feddes Repert. 111(7-8): 543.

$\equiv$ Pleurospermum macrochlaenum Fu Kuntsun et Ho Yehchi, 1979, in Shan Renhwa \& Sheh Menglan (eds.), Fl. Reipubl. Popularis Sin. 55(1): 298, 169, tab. 88 , fig. $1-8$.

Type: CHINA. "Tibet, Chi-lung Hsien, To-tang village, alt. $3500 \mathrm{~m}, 22.06 .1972$, Tibetan medicinal plant collection 397".

Distribution in China: Tibet: Xizang A. R.; SW: Yunnan. Endemic.

Hymenidium mieheanum Pimenov et Kljuykov, 2004, Bot. Zhurn. (St. Petersburg) 89(10): 1661, fig. 8,9 .

Type: CHINA. "Xizang A. R. (Tibet): N side of Kampa La strongly grazed Artemisia-Lonicera dwart-scrub on $\mathrm{N}$-facing moraine slope. $29^{\circ} 20^{\prime} 27$ N, 90³'0 E. 4030 m, 21. 08. 1998, G. et S. Miehe 98-04321" (holotype GOET!).

Distribution in China: Tibet: Xizang A. R. Endemic.

Hymenidium nanum (Rupr.) Pimenov et Kljuykov, 2000, Feddes Repert. 111(7-8): 549.

$\equiv$ Hymenolaena nana Rupr., 1869, Mém. Acad. Imp. Sci. Saint-Petersbourg (ser. 7) 14(4) (Sert. Tiansch.): 50 .

$\equiv$ Hymenolaena lindleyana Klotzsch var. nana (Rupr.) B. Fedtsch., 1911, Beih. Bot. Centralbl. 28(2): 64.

Type: KIRGHIZIA. "Tian-Schan, TaschrobatSchlucht, 04. 08. 1867, Osten-Saken" (lectotype LE!, designated by Vinogradova, 1997b: 54).
Distribution in China: China: NW: Xinjiang Weiwuer A. R.

Distribution outside China: Kazakhstan, Kirghizia, Tajikistan. Endemic to Central Asia.

Hymenidium nubigenum ( $\mathrm{H}$. Wolff) Pimenov et Kljuykov, 2000, Feddes Repert. 111(7-8): 549.

$\equiv$ Pleurospermum nubigenum H. Wolff, 1922, Repert. Spec. Nov. Regni Veg. Beih. 12 (Limpr. Bot. Reise China Ost-Tibet): 448.

Type: CHINA. "Ost-Tibet: Kanse-Dege, Gerollhalden des Passes Tschola. 5150 m, 03. 08. 1914, Limpricht 2149" (holotype WU!).

Distribution in China: Tibet: Xizang A. R.; SW: Sichuan, Yunnan. Endemic.

Hymenidium pachycaule Pimenov et Kljuykov, 1996, Edinburgh J. Bot. 53(2): 275.

Type: CHINA. "Central Kansu. Lien hoa shan: high rocky alpine meadows. Alt. $11500 \mathrm{ft}$., 14-20. 07. 1925, Rock 12703" (holotype E! barcode K000685342; isotypes BM - barcode BM000563961, GH - barcode GH00299256, K!, NAS!).

Distribution in China: NW: Gansu. Endemic.

Hymenidium pilosum (C. B. Clarke ex H. Wolff) Pimenov et Kljuykov, 2000, Feddes Repert. 111(78): 546.

$\equiv$ Pleurospermum pilosum C. B. Clarke ex H. Wolff, 1929, Repert. Spec. Nov. Regni Veg. 27: 117.

Type: CHINA. "Himalaya, Chumbi: Ka-poofo, 28. 07. 1884, Dr. King's collector 70" (holotype K! barcode K000685340; photo E!).

Distribution in China: Tibet: Xizang A. R.

Distribution outside China: Bhutan, India.

Hymenidium pulszkyi (Kanitz) Pimenov et Kljuykov, 2000, Feddes Repert. 111(7-8): 544.

$\equiv$ Pleurospermum pulszkyi Kanitz, 1891, Növényt. Gyujtesek Eredm. Grof Szechenyi Bela Keletazsiai Utjobol.: 25.

Type: CHINA. "Kansu, in jugi Kvetchiensis [Khaji-san-iensis] latere septentr. et merid., 9000 ped., 08. 1879, de Loszy" (holotype BP!).

Distribution in China: NW: Gansu; Tibet: Xizang A. R., Qinghai; SW: Sichuan, Yunnan. Endemic.

Hymenidium sikkimense (C. B. Clarke) Pimenov et Kljuykov, 2000, Feddes Repert. 111(7-8): 545.

$\equiv$ Pleurospermum sikkimense C. B. Clarke, 1879 , in Hooker f. (ed.), Fl. Brit. India 2: 702. 
$\equiv$ Pleurospermopsis sikkimensis (C. B. Clarke) C. Norman, 1938, J. Bot. 76: 200.

Type: INDIA. "Sikkim, alt. 13000-16000 ft. [Yongse], 06. 09. 1849, Hooker" (lectotype K! barcode K000685380, designated by Watson in K; isolectotypes $\mathrm{K}, \mathrm{LE}$ !, P, W!); "Sikkim, Jongri, 15. 10. 1875, Clarke 26164B, 26207A-C, F" (syntypes K).

Distribution in China: Tibet: Xizang A. R.

Distribution outside China: Bhutan, Nepal, India.

Hymenidium stellatum (D. Don) Pimenov et Kljuykov, 2000, Feddes Repert. 111(7-8): 549.

$\equiv$ Selinum stellatum D. Don, 1825 , Prodr. F1. Nepal.: 185 .

$\equiv$ Hymenolaena stellata (D. Don) Lindl., 1835, in Royle, Ill. Bot. Himal. Mts.: 233.

$\equiv$ Pleurospermum stellatum (D. Don) Benth. ex C. B. Clarke, 1879, in Hooker f. (ed.), Fl. Brit. India 2: 704

Type: NEPAL. "Nepal, Buchanan-Hamilton".

= Hymenolaena govaniana DC., 1830, Prodr. 4: 246.

= Pleurospermum govanianum (DC.) Benth. ex C. B. Clarke, 1879, in Hooker f. (ed.), Fl. Brit. India 2: 702 .

Type: INDIA. "In montibus nivosis Sirmore, Govan [546]" (lectotype G-DC!, designated here; isolectotype K-WALLICH!).

Distribution in China: Tibet: Xizang A. R.

Distribution outside China: Nepal, India, Pakistan.

Hymenidium szechenyi (Kanitz) Pimenov et Kljuykov, 2000, Feddes Repert. 111(7-8): 544.

$\equiv$ Pleurospermum szechenyi Kanitz, 1891, in Növényt. Gyujtesek Eredm. Grof Szechenyi Bela Keletazsiai Utjobol.: 25.

Type: CHINA. "Kansu, in jugi Khaji-san-iensis, latere septentr. et merid., 08. 1879, de Loszy" (holotype BP, probably lost).

Distribution in China: NW: Gansu; Tibet: Xizang A. R., Qinghai; SW: Sichuan. Endemic.

Hymenidium tsekuense (Shan Renhwa ex Liou Shoulu) Pimenov et Kljuykov, 2000, Feddes Repert. 111(7-8): 545.

$\equiv$ Pleurospermum tsekuense Shan Renhwa ex Liou Shoulu, 1979, in Shan Renhwa \& Sheh Menglan (eds.), Fl. Reipubl. Popularis Sin. 55(1): 298, 143, tab. 71, fig. 1-5.
Type: CHINA. "Chinghai, Tseku Hsien, L. W. Chou et L. L. Sun 1396".

Distribution in China: Tibet: Qinghai. Endemic.

Hymenidium virgatum Pimenov et Kljuykov, 2004, Bot. Zhurn. (St. Petersburg) 89(10): 1654, fig. 2,3 .

Type: CHINA. "Sichuan, Kanding Co., pass across Daxue Shan between Xinduquao and Kanding, E of Shuizheotzi Lat. 30 04' N, Long. $101^{\circ} 46^{\prime}$ E. Alt. 4340 m, 19. 09. 1998, Pimenov, Kljuykov, Hu Zhihao and Liu Qixing 258" (holotype MW!; isotype LE!).

- Ligusticum franchetii auct. non H. Boissieu: Pu Fating, 1991, Acta Phytotax. Sin. 29(6): 541, fig. 8.

Distribution in China: SW: Sichuan. Endemic.

Hymenidium wilsonii ( $\mathrm{H}$. Boissieu) Pimenov et Kljuykov, 2000, Feddes Repert. 111(7-8): 549.

$\equiv$ Pleurospermum wilsonii H. Boissieu, 1906, Bull. Soc. Bot. France 53: 433.

Type: CHINA. "Western China, 14000 ft., 09. 1904, Wilson 3682" (lectotype P! - barcode P00834554, designated by Pimenov, Kljuykov, 2000: 549; isolectotypes A - barcode A00062640, HBG - barcode HBG510910, K! - barcode K000685336); "Thibet oriental, 07. 1894, Soulié 2185" (syntype P!).

= Pleurospermum wilsonii $\mathrm{H}$. Boissieu var. foliosum H. Boissieu, 1906, Bull. Soc. Bot. France 53: 433.

Type: CHINA. "Ta-Tsien-Lou, 1894, Soulié" (holotype P?).

= Pleurospermum crassicaule H. Wolff, 1925, Repert. Spec. Nov. Regni Veg. 21: 241.

Type: CHINA. "Tibetia orientalis, loco non indicato, 07. 1894, Soulié 2185" (holotype P! barcode P00834555).

= Pleurospermum cnidiifolium H. Wolff, 1926, Acta Horti Gothob. 2: 292.

Type: CHINA. "Sze-ch'uan, reg. bor.: Huangch'en-kuan, orc. versus in calicatis herbosis, 3300 m, 19. 08. 1922, H. Smith 4068" (syntypes PE, UPS!); “Sze-ch'uan, reg. bor--occid.: Dalgang cia. $50 \mathrm{~km}$ VSV von Merge, $3500 \mathrm{~m}, 03$. 09. 1922, H. Smith 4313" (lectotype GB! - barcode GB0048821, designated by Pimenov, Kljuykov, 2000: 550; isolectotype UPS!).

= Pleurospermum lecomteanum H. Wolff, 1929, Repert. Spec. Nov. Regni Veg. 27: 116.

Type: CHINA. "Su-tchuen: Bassin de Tongho (M.Thibet), Dzenla, roches metamorph., 3500 
m, prairies alpines, 24. 09. 1911, Legendre 1537" (lectotype P! - barcode P00834556, designated by Pimenov, Kljuykov, 2000: 550); "Su-tchuen: Bassin de Tong-ho (M.Thibet) Tse minuda, terrain schistos., 4500 m, 04.10.1911, Legendre 1603" (syntype P! barcode P00834557).

Distribution in China: Tibet: Xizang A. R., Qinghai; SW: Sichuan.

\section{Distribution outside China: Nepal.}

Hymenidium wrightianum ( $\mathrm{H}$. Boissieu) Pimenov et Kljuykov, 2000, Feddes Repert. 111(78): 544.

$\equiv$ Pleurospermum wrightianum H. Boissieu, 1903, Bull. Herb. Boissier (2 sér.) 3(10): 847.

Type: CHINA. "Thibet oriental: Ta-Tsien-Lou, Principalité de Kia-La [Tongolo, bord des champes, pelouses riches], 06. 07. 1892, Soulié 467" (lectotype P! - barcode P00834558; isolectotype P - barcode P00834559).

= Pleurospermum prattii $\mathrm{H}$. Wolff, 1929, Repert. Spec. Nov. Regni Veg. 27: 118.

Type: CHINA. "West Szechuan et Tibetian frontier: chiefly near Tachienlu, at 9000-13000 feet, Pratt 566" (holotype K! - barcode K000685344).

Distribution in China: Tibet: Xizang A. R., Qinghai; SW: Sichuan, Yunnan. Endemic.

Hymenidium yunnanense (Franch.) Pimenov et Kljuykov, 2000, Feddes Repert. 111(7-8): 550.

$\equiv$ Pleurospermum yunnanense Franch., 1894, Bull. Annuel Soc. Philom. Paris (sér. 8) 6: 137.

Type: CHINA. "Province du Yun-nan, in dumetis supra Kan-ho et Fong-yang-tchang, alt. 3500 m, 08. 08. 1888, Delavay 3386" (syntypes GH barcode GH00062638, P! - barcodes P00834568, P00834569, P00834570); "Province du Yunnan, in herbosis montis Tsang-chan, alt. $4000 \mathrm{~m}$. [Lieux herbeux sur le Tsang chan, au dessus de Tali, a 4000 m], 19. 08. 1887, Delavay 2655, 3173" (lectotype P! - barcode P00834562, designated by Pimenov, Kljuykov, 2000: 550; isolectotypes K! barcode K000685334; P! - barcodes P008345643, P00834564, P00834565, P00834566, P00834567).

= Pleurospermum pseudoyunnanense $\mathrm{H}$. Wolff, 1929, Repert. Spec. Nov. Regni Veg. 27: 118.

Type: CHINA. "Yunnan [Thibet]: Tsekou, Manberg" (lectotype P! - barcode P00834571, designated here; isolectotype $\mathrm{K}$ ! - barcode K000685335).

Distribution in China: SW: Sichuan, Yunnan.

Distribution outside China: Myanmar.
Kadenia salina (Turcz.) Lavrova et V. N. Tikhom., 1986, Byull. Moskovsk. Obšč. Isp. Prir. Otd. Biol. 91(2): 93.

$\equiv$ Cnidium salinum Turcz., 1844, Bull. Soc. Imp. Naturalistes Moscou 17: 733.

Type: RUSSIA. "In salsis Dahuriae atque prope salinas Selenginenses [Salinae Selenginenses], 1829, Turczaninow" (lectotype LE!, designated by Vinogradova, 2005: 961; isolectotypes KW!, LE!, M - barcode M0172890, P! - barcodes P00758148, P03224457, P03224458, P03237375).

=Ligusticum tibetanicum $\mathrm{H}$. Wolff, 1930, Repert. Spec. Nov. Regni Veg. 27: 317.

Type: CHINA. "Tibet altissima: Kokonor. K. Futterer, Holderer 96" (syntype); "China, Kansu, ca. 4800 m s.m., 10. 09. 1918, Licent 4810" (lectotype $\mathrm{P}$ !, designated by Pimenov in Pimenov, Ostroumova, 2012: 220; isolectotypes PE!, W!); "Kokonor, 10. 08. 1923, Andersson 1215" (syntype S! - barcode S12-21686).

= Cnidium salinum auct. non Turcz.: Liou Shoulu et al. var. rhizomaticum Ma Yuchuan, 1979, Fl. Intramong. 4: 207, 179.

Type: CHINA. "Nei Mongghol, Uxin Qi, Ih Ju Meng, meadows among hills, Mong. Medic. Exped. 271" (holotype HIMC).

Distribution in China: NW: Xinjiang Weiwuer A. R., Gansu; Tibet: Qinghai; N: Neimenggu A. R., Ningxia Huizu A. R., Hebei; NE: Heilongjiang.

Distribution outside China: Russia, Mongolia.

Kailashia xizangensis (Chang Hotseng et Shan Renhwa) Pimenov et Kljuykov, 2005, Feddes Repert. 116(1-2): 84.

$\equiv$ Pachypleurum xizangense Chang Hotseng et Shan Renhwa, 1980, Acta Phytotax. Sin. 18 (3): 376.

Type: CHINA. "Xizang: Burang Xian, alt. 4600 m, 24. 08. 1974, Xizang Exped. (NW Plateau Inst. Biol.) 4223" (holotype HNWP; isotype XJBI!).

Distribution in China: Tibet: Xizang A. R. Endemic.

Keraymonia cortiformis Cauwet et S. B. Malla, 1985, Candollea 40(2): 531, fig. 6 (6-9).

Type: NEPAL. "C. Nepal: haute Kali Gandaki, entre Muktinath et Thorung-la, vers 4900-5000 m, 19. 09. 1981, Farille 81456B" (holotype KATH; isotype E! - barcode E00000246).

Distribution in China: Tibet: Xizang A. R.

Distribution outside China: Nepal.

Kitagawia baicalensis (Redow. ex Willd.) Pimenov, 1986, Bot. Zhurn. (Leningrad) 71(7): 944. 
$\equiv$ Selinum baicalense Redow. ex Willd., 1809, Enum. P1.: 306.

$\equiv$ Peucedanum baicalense (Redow. ex Willd.) W. D. J. Koch, 1824, Nova Acta Phys.-Med. Acad. Caes. Leop.-Carol. Nat. Cur. 12, 1: 94.

Type: RUSSIA. "Culta in H.B. Berol. [Origin: ad lacum Baical. Redowsky [5719]" (holotype B-Willd! - barcode B-W 05719 -01 0).

= Selinum obscurum Fisch. ex Trev., 1821, Index Sem. Horti Wratisl. App. 3: 3.

= Peucedanum polyphyllum Ledeb., 1829, Fl. Altaic. 1: 314.

$=$ Peucedanum polyphyllum Ledeb. var. pubescens Ledeb., 1829, Fl. Altaic. 1: 310.

Type: KAZAKHSTAN. "Altai. In rupestribus siccis ad fl. Tscharysch et Kerlyk [In rupestribus ad fl. Tscharysch]. 1826, Ledebour 848" (lectotype LE!, designated by Vinogradova, 2005: 964; isolectotypes CGE!, HAL - barcode HAL0098500, $\mathrm{P}$ - barcodes P007311609, P007311610, P03223233, P03223234, P03223236, P03223238, P03223240); "prope Riddersk, Loktewsk et alibi” (L); "in collibus arenosis circa Schulbinsk ad fl. Irtysch" (M).

= Peucedanum polyphyllum Ledeb. var. glabrum Ledeb., 1829, Fl. Altaic. 1: 310.

Type: KAZAKHSTAN/RUSSIA. "In sabulosis prope Schubinsk (M.) et Loktewsk (L.) [In collibus arenosis pinetis inter redout Schubinsk et Oserki non procul a fortalintis Semipalatinsk. 09. 07. 1826. Meyer" (lectotype LE!, designated by Vinogradova, 2005: 964). RUSSIA. "Prope Loktevsk (L.), Ledebour 1195" (syntype LE!).

$=$ Peucedanum polyphyllum Ledeb. var. lineare Ledeb., 1829, Fl. Altaic. 1: 310.

Type: KAZAKHSTAN. "In rupestribus siccis prope Ridersk [Pr. Riddersk], Ledebour" (holotype LE!).

= Peucedanum caespitosum H. Wolff, 1926, Acta Horti Gothob. 2: 323.

Type: CHINA. "Chili: Hsiao-wu-tai-shan, Tienlin-ssu, Uferabhang. Ad rivulum in declivitate aprica, lapidosa; strandbrink, 23. 09. 1921, H. Smith 66" (lectotype UPS!, designated here; isolectotype GB!barcode GB-0048816).

= Peucedanum trinioides $\mathrm{H}$. Wolff, 1926, Acta Horti Gothob. 2: 325.

Type: CHINA. "Chili: Hsiao-wu-tai-shan, Yangkia-p'ing, Tung-lin. auf Gebirgstriften, 1700-1900 m, 27. 08. 1921, H. Smith 452" (syntypes BM barcode BM000885398, GB! - barcode GB0048818, UPS!); “Chili: zwischen Tao-lai-shui und
Kuo-che-wai, 1300-1600 m, 15. 09. 1921, H. Smith 364" (lectotype UPS!, designated here by Pimenov).

Distribution in China: (N: Neimenggu A. R., Shanxi, Hebei; NE: Heilongjiang).

Distribution outside China: Russia, Mongolia, Kazakhstan.

Kitagawia formosana (Hayata) Pimenov, comb. nov.

$\equiv$ Peucedanum formosanum Hayata, 1921, Icon. P1. Formosan. 10: 22, tab. 13.

Type: CHINA. "[Taiwan] Mt. Niitaka, ad 10000 ped. alt. [Nanto], 19. 10. 1906, Kawakami, Mori 2052" (holotype TAIF).

Distribution in China: Centr.: Jiangxi, Fujiang; S: Taiwan, Guangxi Zhuang A.R., Guangdong. Endemic.

Kitagawia komarovii Pimenov, 1986, Bot. Zhurn. (Leningrad) 71(7): 948.

三 Peucedanum elegans Kom. 1901, Trudy Imp. S.-Peterburgsk. Bot. Sada 18: 430, nom. illeg. (Art. 53.1), non Sweet (1830).

Type: KOREA. "In saxosis montium secus fluvii Jalu-dsian decursum superiorem saepe crescit. Karami ad ripam sinistram, 25. 07. 1896, Komarov 1295" (lectotype LE!, designated by Pimenov, 1986: 948, as "typus"; isolectotypes K! - barcode P02272007, LE!, P); "In saxosis montium secus fluvii Jalu-dsian decursum superiorem saepe crescit. Ad ripam dextram paulum infra ostium fl. Czandshin-gan, 13. 08. 1896, Komarov" (syntypes K, LE!).

= Peucedanum hakuunense Nakai, 1939, J. Jap. Bot. 15: 740 .

Type: KOREA. "Prov. Zennan: in herbidis montis Hakuunzan, 20. 08. 1934, Nakai" (holotype TI!).

Distribution in China: N: Hebei; NE: Heilongjiang, Jilin, Liaoning.

Distribution outside China: Russia, Korea.

Kitagawia macilenta (Franch.) Pimenov, comb. nov.

$\equiv$ Peucedanum macilentum Franch., 1894, Bull. Annuel Soc. Philom. Paris (sér. 8) 6: 142.

Type: CHINA. "Province du Yun-nan, Hokin, prope Koua-la-po, alt. $2800 \mathrm{~m}, 18$. 08. 1886, Delavay 2476" (lectotype P! - barcode P02272026, designated here; isolectotypes $\mathrm{K}$ ! - barcode K000685420, NY - barcode NY00406176, P barcode P02272027). 
= Ligusticum glaucescens Franch., 1894, Bull. Annuel Soc. Philom. Paris (sér. 8) 6: 134.

Type: CHINA. "Yunnan, in pratis adumbratis prope Mo-che-tschin, ad cataractas supra Tapin-tze [près de la cascade du Mo-che-tsin, au-dessus de Ta-pin-tze], 03. 10. 1882, Delavay" (syntype P! barcodes P03224789, P03224792, P03224793, P03224795); "Yunnan, in pratis montis Pee-ngaytze, supra Tapin-tze [prairie du Mt Pee ngay tze audessus de Ta-pin tze], 01. 09. 1882, Delavay 446" (lectotype P! - barcode P03224790, designated here; isolectotype $\mathrm{P}$ - barcode P03224794, syntype P!).

Distribution in China: China (SW: Sichuan, Yunnan).

Distribution outside China: Endemic to Eastern Asia.

Kitagawia pilifera (Hand.-Mazz.) Pimenov, comb. nov.

$\equiv$ Peucedanum piliferum Hand. Mazz., 1933, Oesterr. Bot. Z. 82: 252.

Type: CHINA. "SE Mandschurei, TygroveGebiet, auf Wiesen, 350 m, 03. 08. 1931, Fenzel 352" (holotype W!).

Distribution in China: China (NE).

Distribution outside China: Endemic to Eastern Asia.

\section{nov.}

Kitagawia praeruptora (Dunn) Pimenov, comb.

$\equiv$ Peucedanum praeruptorum Dunn, 1903, J. Linn. Soc. Bot. 35: 497.

Type: CHINA. "Hupeh, Ichang, 1887. Henry 2911" (syntypes K!, P - barcodes P02272036, P02272037); "Changyang, Henry 7505“ (syntype K!); "Szechuan, North Wushan, 07. 1888, Henry 7475 " (lectotype E! - barcodes E00002618, E00002619, designated here; isolectotypes BM! - barcode BM000885390, G!, K! - barcodes K000685408, K000685409, K000685411, K000685412， P - barcodes P022722038, $\mathrm{P} 022722039)$.

= Peucedanum turgeniifolium H. Wolff, 1926, Acta Horti Gothob. 2: 323.

Type: CHINA. "Sze-ch'uan bor-occid.: Ch'osodjo, Ö om älven. Torr buskäng, 18. 10. 1922, H. Smith 4826" (lectotype UPS!, designated here; isolectotype GB!).

= Peucedanum pulchrum H. Wolff, 1926, Acta Horti Gothob. 2: 324.

Type: CHINA. "Sze-ch'uan, reg. bor.: ad austro-occid. versus a Sung-pan åt SV, alt. 3900 m. Torr ängsmark, in prato aprico, 30. 07. 1922, H. Smith 3743" (lectotype UPS!, designated here; isolectotypes BM! - barcode BM000885420, GB! barcode GB-0048817, LD! - barcode LD1042582, $\mathrm{W}$ !).

Distribution in China: NW: Xinjiang Weiwuer A. R., Gansu; Tibet: Xizang A. R., Qinghai; N: Ningxia Huizu A.R., Shaanxi, Shanxi; Centr.: Henan, Jiangsu, Anhui, Hubei, Hunan, Jiangxi, Zhejiang, Fujiang; SW: Sichuan, Guizhou; S: Guangxi Zhuang A. R., Guangdong. Endemic.

Kitagawia stepposa (Huang Yhuei) Pimenov, comb. nov.

三Peucedanum stepposum Huang Yhuei, 1977, Fl. Pl. Herb. Chin. Bor.-Or. 6: 273, 294, tab. 112, fig. 1-5.

Type: CHINA. "Jilin, Shuangliao Xian, Zhengjiatun, 09. 09. 1938, coll. unclear 6931" (holotype IFP).

- Cnidium salinum auct. non Turcz.: Liou Shoulu et al., 1959, Clav. Pl. Chin. Bor.-Orient.: 256, tab. 82, fig. 5.

Distribution in China: N: Neimenggu A. R.; NE: Heilongjiang, Jilin, Liaoning. Endemic.

Kitagawia terebinthacea (Fisch. ex Trev.) Pimenov, 1986, Bot. Zhurn. (Leningrad) 71(7): 944.

इSelinum terebinthaceum Fisch. ex Trev., 1821, Ind. Sem. Hort. Vratisl. App. 3: [3].

$\equiv$ Peucedanum terebinthaceum (Fisch. ex Trev.) Ledeb., 1844, Fl. Ross. 2(1): 314.

Type: RUSSIA. "Sibir".

= Oreoselinum dahuricum Besser ex Steud., 1841, Nomencl. Bot. (ed. 2) 2: 226.

= Peucedanum davuricum Turcz. ex Ledeb., 1844, Fl. Ross. 2: 314.

Type: RUSSIA. "In pratis Dahuriae, 1831, Turczaninow" (lectotype LE?; isolectotypes K!, P! barcode P03227748).

= Peucedanum deltoideum Makino ex Yabe, 1902, J. Coll. Sci. Imp. Univ. Tokyo 16(4) (Rev. Umbell. Jap.): 99, tab. 3, fig. 60.

= Peucedanum terebinthaceum (Fisch. ex Trev.) Ledeb. var. deltoideum (Makino ex Yabe) Makino, 1907, in Iinuma (ed.), Somoku-dzusetsu (3rd ed.) 1: 357, tab. 10.

= Peucedanum terebinthaceum (Fisch. ex Trev.) Ledeb. subsp. deltoideum (Makino ex Yabe) Vorosch., 1985, in Skvortzov (ed.), Florist. issl. v razn reionakh SSSR: 184.

Type: JAPAN. "Prov. Omi: in monte Ibukiyama, Tsuji" (syntype); "Shikoku, in pago Kamibun. Makino" (syntype MAK?). 
$=$ Seseli libanotis (L.) W. D. J. Koch var. kurilense Takeda, 1914, J. Linn. Soc. Bot. 42: 467.

$=$ Libanotis ugoensis (Koidz.) Kitag. var. kurilensis (Takeda) T. Yamaz., 2001, J. Jap. Bot. 76(5): 283.

Type: RUSSIA. "Kuril Islands, Shikotan, Anama, in grassy places [on grassy slopes], Takeda" (holotype ?)

= Peucedanum paishanense Nakai, 1917, Bot. Mag. (Tokyo) 31: 101.

= Peucedanum terebinthaceum (Fisch. ex Trev.) Ledeb. var. paishanense (Nakai) Huang Yhuei, 1977, Fl. Pl. Herb. Chin. Bor.-Or. 6: 277.

Type: KOREA. "Korea sept.: Districtu montis Paik-tu-san: in silvis Laricis inter Nong-sa-dong et Mu-bon, 13. 08. 1914, Nakai 4012" (lectotype TI!, designated by Pimenov in Pimenov, Ostroumova, 2012: 299); "Korea sept.: in silvis Laricis inter Hohang-ryöng et Shin-mu-jyang, Nakai 4013" (syntype TI); "In silvis circa Paik-tu-san, Mori 119, 162" (syntype TI?); "Korea media: in rupibus montium Kum-gang-san, Nakai 5721" (syntype TI?).

= Peucedanum terebinthaceum (Fisch. ex Trev.) Ledeb. var. flagellare Nakai, 1917, Bot. Mag. (Tokyo) 31: 101.

Type: JAPAN. "Yezo, Horomanbetsu prov. Hidaka, Tokubuchi" (syntype); "Yezo, Toyohiragawara circa Sapporo prov. Ishikari, Matsumura" (syntype); "KOREA. In monte circa Ouensan, 07. 06. 1909, Nakai" (syntype TI!); "Musang, Mori 319" (syntype).

= Peucedanum fauriei Fedde ex H. Wolff, 1934, Repert. Spec. Nov. Regni Veg. 33: 250.

Type: KOREA. "Quelpaert, in rupestribus litoralibus prope Mong no, 02. 11. 1906, Faurie 1020" (lectotype P! - barcode P02272045, designated here; isolectotypes BM!, E!, P!).

= Peucedanum terebinthaceum (Fisch. ex Trev.) Ledeb. var. aciculare T. Inoue, 1957, J. Jap. Bot. 32(6).

= Peucedanum terebinthaceum (Fisch. ex Trev.) Ledeb. f. aciculare (T. Inoue) H.Ohba, 1999, in Iwatsuki et al. (eds.), Fl. Jap. IIc: 301.

Type: JAPAN. "[Hokkaido] Prov. Ishikari: in saxo septentrionalis partis montis Moiwa, 14. 10. 1932, Inoue" (holotype Herb. Inoue).

Distribution in China: N: Neimenggu A. R., Hebei; NE: Heilongjiang, Jilin, Liaoning; Centr.: Henan.

Distribution outside China: Russia, Korea, Mongolia, Japan (Hokkaido; Honshu; Shikoku; Kyushu).
Krasnovia longiloba (Kar. et Kir.) Popov, 1950, in Schischkin (ed.), Fl. URSS 16: 118, 592, tab. 15, fig. 1.

इSphallerocarpus longilobus Kar. et Kir., 1841, Bull. Soc. Imp. Naturalistes Moscou 14: 432.

$\equiv$ Chaerophyllum sphallerocarpus Kar. et Kir., 1842, Bull. Soc. Imp. Naturalistes Moscou 15: 367, nom. illeg. (Art. 52.1).

$\equiv$ Chaerophyllum longilobum (Kar. et Kir.) O. et B. Fedtsch., 1909, Consp. Fl. Turkest. 3: 120.

$\equiv$ Conopodium longilobum (Kar. et Kir.) KosoPol., 1916, Bull. Soc. Imp. Naturalistes Moscou, s.n. 29: 206.

$\equiv$ Kozlovia longiloba (Kar. et Kir.) Spalik et S. R. Downie, 2001, Ann. Missouri Bot. Gard. 88(2): 289.

Type: KAZAKHSTAN. "In rupestribus montium Tarbagatai ad torrentem Dschanybek, 1840, Karelin, Kirilov 58" (lectotype LE!, designated by Vinogradova, 2002b: 137).

Distribution in China: NW: Xinjiang Weiwuer A. R.

Distribution outside China: Kazakhstan, Kirghizia.

Ledebouriella multiflora (Ledeb.) H. Wolff, 1910, in Engler (ed.), Pflanzenreich 43(IV, 228): 191.

$\equiv$ Rumia multiflora Ledeb., 1844, Fl. Ross. 2: 281.

Type: KAZAKHSTAN. "[Altai?] In montibus ... Deserti Kirghisici ... Sievers, 1623 (10)" (holotype B-Willd, see Vinogradova (2007: 270); isotype LE!).

= Stenocoelium tenuifolium Korovin, 1962, Trudy Inst. Bot. Akad. Nauk Kazakh. SSR 13: 256.

Type: KAZAKHSTAN. "Altai, mons Mramornaja, ad declivitatem lepidosam non procul pag. Alexeevka, 17. 07. 1958, Stepanova" (syntype AA!); "Tarbagatai, in angustis riv. Tschitlosty, alt. 1200 m, ad locos lapidosos, 19. 07. 1955, Stepanova" (syntype AA!).

Distribution in China: NW: Xinjiang Weiwuer A. R.

Distribution outside China: Kazakhstan.

Levisticum officinale W. D. J. Koch, 1824, Nova Acta Phys.-Med. Acad. Caes. Leop.-Carol. Nat. Cur. 12, 1: 101, fig. 41.

$\equiv$ Ligusticum levisticum L., 1753, Sp. pl.: 250.

三Angelica levisticum (L.) All., 1785, Fl. Pedem.

2: 10 .

三Levisticum levisticum (L.) Karst., 1882, Deut. Fl. 9: 844, nom. inval. (Art. 23.4). 
$\equiv$ Levisticum vulgare Bubani, 1900, Fl. Pyren. 2: 384, nom. illeg. (Art. 52.1).

$\equiv$ Selinum levisticum (L.) E. H. L. Krause, 1904, in Sturm (ed.), Deutschl. Fl. (ed. 2) 12: 116.

$\equiv$ Hipposelinum levisticum Britt., 1913, in Britton \& Brown (eds.), Ill. Fl. North Un. St. (ed. 2) 2: 635, fig. 3124.

Type: EUROPE, ITALY. "In Apenninis Liguriae, 97, Ligusticum 2" (lectotype BM-Cliff, designated by Reduron in Jarvis et al., 2006: 213).

$=$ Levisticum persicum Freyn et Bornm., 1897, Bull. Herb. Boissier 5: 619.

$=$ Levisticum officinale W. D. J. Koch subsp. persicum (Freyn et Bornm.) Thell., 1923, Verh. Naturf. Ges. Basel 35(1): 33.

Type: IRAN. "Persiae austr. provinc. Kerman, in monte Kuh-i Häsar inter urbem Kerman et Bender Abbas, 3400 m. supra mare, 10.08. 1892, Bornmüller 3834" (lectotype BRNM!, designated by Pimenov, Sutorý (2014: 1107); isolectotypes B!, BM! - barcode BM000944778, BP!, E! - barcodes E00002377, E00002378, G - barcode G00366976, JE - barcode JE00007587, K! - barcode K001097171, LD! - barcode LD1420437, MPU - barcode MPU020978, W barcode W18970001256, Z).

Distribution in China: N: Neimenggu A. R., Shaanxi, Shanxi, Hebei, Shandong; NE: Liaoning; Centr.: Henan, Fujiang. In China the species is cultivated.

Distribution outside China: Russia, Afghanistan, Iran, Europe, N. America.

Libanotis laoshanensis Zhou Wei et Liu Qixin, 2015, Plant Resources Environm. 24(3): 107.

Type: CHINA. "Jiangsu, Jiangpu in Nanjing, 400 m. 01. 08. 1964. Liu S. L. et al. 1670" (holotype NAS).

Distribution in China: Centr.: Jiangsu. Endemic.

Note: Recently described and little known species, probably related to Seseli seseloides.

Ligusticopsis acaulis (Shan Renhwa et Sheh Menglan) Pimenov, comb. nov.

$=$ Peucedanum acaule Shan Renhwa et Sheh Menglan, 1986, Acta Phytotax. Sin. 24(4): 308.

Type: CHINA. "Yunnan: Huize, alt. 3450 [3500] m, 26. 09. 1963, Exped. Dangchuan 63-014" (holotype KUN!; isotype NAS!).

Distribution in China: SW: Yunnan. Endemic.

Ligusticopsis brachyloba (Franch.) Leute, 1969, Ann. Naturhist. Mus. Wien 73: 71, tab. 3, fig. 3. $\equiv$ Ligusticum brachylobum Franch., 1894, Bull. Annuel Soc. Philom. Paris (sér. 8) 6: 134.

Type: CHINA. "Collines calcareis au dessus de Mo-so-yn, 10. 08. 1889, Delavay 4092" (lectotype P! - barcode P03224546, designated by Leute, 1969: 71); "Côteaux calcaires au pied du Yang-in-chan (Lankong), à $2500 \mathrm{~m}, 06.08 .1886$, Delavay 2466" (syntype P! - barcodes P03237446, P03237447); "Côteaux calcarais au pied du Yangin-chan (Lankong), 15. 09. 1885, Delavay 2027" (syntypes K, P! - barcodes P03224548, P03237441, P03237445).

= Peucedanum cavaleriei $\mathrm{H}$. Wolff, 1925, Repert. Spec. Nov. Regni Veg. 21: 246.

Type: CHINA. "Prov. Yunnan, reg. bot. Yunnanfu, Cavalerie" (holotype P!).

Distribution in China: Tibet: Xizang A. R., Qinghai; N: Shanxi, Shaanxi; Centr.: Hubei; SW: Sichuan, Guizhou, Yunnan. Endemic.

Ligusticopsis capillacea Leute, 1969, Ann. Naturhist. Mus. Wien 73: 71, tab. 3, fig. e.

三Ligusticum capillaceum H. Wolff, 1930, Repert. Spec. Nov. Regni Veg. 27: 311, nom. illeg. (Art. 53.1), non Lam. (1779).

$\equiv$ Pleurospermum capillaceum (H. Wolff) M. Hiroe, 1958, Umbell. Asia 1: 122.

Type: CHINA. "Yunnan: eastern slopes of Likiang Snow Range, Yangtze watershed, 15000 ft., limestone gravel, 19. 09. 1923, Rock 10791" (lectotype US! - barcode US00611150, designated here; isolectotypes E! - barcode E00000230, P!, UC!, US!); "Yunnan, Yangtze watershed, prefectural district of Likiang, eastern slopes of Likiang Snow Range, 01. 08. 1922, Rock 5266" (syntypes UC!, US! - barcode US00611149); "Yunnan, eastern slopes of Mount Dyinaloko, northern peak of the Likiang Snow Range, 14-15000 ft. s.m., 08. 1923, Rock 10378" (syntypes P!, UC!, US).

Distribution in China: SW: Sichuan, Yunnan. Endemic.

Ligusticopsis cryptotaenia (H. Boissieu) Lavrova, 1998, Abstr. II(IX) Meet. Russ. Bot. Soc. 2: 167. "cryptotaenium".

$\equiv$ Selinum cryptotaenium H. Boissieu, 1903, Bull. Herb. Boissier (2 sér.) 3(10): 847.

Type: CHINA. "Les prairies du Yo-lin-Chan, 08. 1893, Delavay 6722" (holotype P!).

Distribution in China: SW: Yunnan. Endemic.

Ligusticopsis daucoides (Franch.) Lavrova [1996, Mat. IX Moscow Conf. Phylogenet. Plant.: 
78, comb. inval. Art. 33.3)] ex Pimenov et Kljuykov, 2004, Bot. Zhurn. (St. Petersbung) 89: 1654.

$\equiv$ Trachydium daucoides Franch., 1885, Nouv.

Arch. Mus. Hist. Nat. (sér. 2) 8 (Pl. David. 2): 245.

$\equiv$ Ligusticum daucoides (Franch.) Franch., 1894,

Bull. Annuel Soc. Philom. Paris (sér. 8) 6: 135.

$\equiv$ Angelica daucoides (Franch.) M. Hiroe, 1958, Umbell. Asia 1: 173.

Type: CHINA. "Thibet oriental, Moupine, in pratis montium altissimarum [haute prairie], 08 . 1869, David" (holotype P! - barcode P03224855).

= Selinum oliverianum H. Boissieu, 1903, Bull.

Herb. Boissier (2 sér.) 3(10): 846.

$=$ Ligusticum oliverianum $(\mathrm{H}$. Boissieu) Shan Renhwa, 1941, Sinensia 12: 175.

Type: CHINA. "Su-Tchuen oriental, district de Tchen-Kéou-Tin, Farges" (lectotype P!, designated here; isolectotypes $\mathrm{P}$ !).

= Ligusticum daucoides (Franch.) Franch. var. souliei H. Boissieu, 1903, Bull. Herb. Boissier (2 sér.) 3(11): 845.

= Ligusticum franchetii $\mathrm{H}$. Boissieu, 1906, Bull. Soc. Bot. France 53: 432.

$=$ Ligusticopsis franchetii $(\mathrm{H}$. Boissieu) Leute, 1969, Ann. Naturhist. Mus. Wien 73: 72, tab. 3, fig. 4.

Type: CHINA. "Thibet oriental: Tse Kou (Haut Mekong), 1893(5?), Soulié 1065” (lectotype P! barcode P04197628; isolectotypes $\mathrm{P}$ - barcodes P03224801, P03224802).

= Ligusticum mairei M. Hiroe, 1958, Umbell. Asia 1: 108.

Type: CHINA. "Prov. Yunnan: pâturages des montagnes arides de Pe-long-tsin, alt. $3300 \mathrm{~m}$, Maire 7371" (holotype UC! - barcode UC385796).

Distribution in China: Tibet: Xizang A. R.; Centr.: Hubei; SW: Sichuan, Guizhou, Yunnan. Endemic.

Ligusticopsis deqenensis (Shan Renhwa et Pan Zehui) Pimenov, comb. nov.

$\equiv$ Heracleum deqenense Shan Renhwa et Pan Zehui, 1997, in Wu Zhengyi (ed.), Fl. Yunnan. 7: 629, tab. 204, fig. 1-4.

Type: CHINA. "Yunnan, Deqen, alt. 4300 m, 1984, C. Q. Yuan 84-135" (holotype HJ).

Distribution in China: SW: Yunnan. Endemic.

Ligusticopsis dielsiana (H. Wolff) Pimenov et Kljuykov, 1999, Feddes Repert. 110(7-8): 484.

三Ligusticum dielsianum H. Wolff, 1930, Repert. Spec. Nov. Regni Veg. 27: 323.

Type: CHINA. "Yunnan: pâturages plateau de Ta-hai, 3200 m, 1913, Maire 1027” (lectotype E! - barcode E00002346, designated here; isolectotype $\mathrm{G} !)$.

= Ligusticopsis rechingerana Leute, 1969, Ann. Naturhist. Mus. Wien 73: 75, tab. 4, fig. 3.

= Ligusticum rechingeranum (Leute) Shan Renhwa et Pu Fating, Acta Phytotax. Sin. 29(6): 544. 1991.

Type: CHINA. "Prov. Yunnan bor.-occid.: prope urbem Lidjiang (Likiang), imprimis in monte Yulung-schan, collectores indigeni 3845" (holotype $\mathrm{W}$ !; isotype $\mathrm{WU}$ !).

Distribution in China: SW: Sichuan, Yunnan. Endemic.

Ligusticopsis hispida (Franch.) Lavrova et Kljuykov, 1994, Bot. Zhurn. (St. Petersburg) 79(10): 105.

$\equiv$ Trachydium hispidum Franch., 1894, Bull. Annuel Soc. Philom. Paris (sér. 8) 6: 113.

$\equiv$ Ligusticum hispidum (Franch.) H. Wolff ex Hand.-Mazz., 1933, Symb. Sin. 7, 3: 723.

Type: CHINA. "Yun-nan, in pratis ad cacumina montis Ki-chan, alt. $2800 \mathrm{~m}$ supra Tali [Prairies montagn., montagne de Kichan près de Ta-li], 10. 09. 1884, Delavay 180" (lectotype P! - barcode P02284821, designated here; isolectotypes NY! barcode NY00406295, P! - barcode P03224777).

= Trachydium rockii $\mathrm{H}$. Wolff, 1929, Repert. Spec. Nov. Regni Veg. 27: 123.

Type: CHINA. "Yunnan, Yangtze watershed, prefectural district of Likiang, eastern slopes of Likiang Snow range, below Mahoang Patze, 11500 ft., 27. 08. 1922, Rock 6075" (holotype US!).

= Ligusticum changii M. Hiroe, 1958, Umbell. Asia 1: 112.

Type: CHINA. "Prov. Yunnan: rocky place, alt. $4000 \mathrm{~m}, \mathrm{Wu}$ Mong mountain, 01. 11. 1940, Chang 635" (holotype UC! - barcode UC792328; isotype PE!).

Distribution in China: SW: Sichuan, Yunnan. Endemic.

Ligusticopsis integrifolia (H. Wolff) Leute, 1969, Ann. Naturhist. Mus. Wien 73: 77, tab. 5, fig. 1.

$\equiv$ Ligusticum integrifolium H. Wolff, 1930, Repert. Spec. Nov. Regni Veg. 27: 307.

Type: CHINA. "Yunnan, eastern flank of the Likiang Range. Lat. $27^{\circ} 30^{\prime}$. Alt. $11000 \mathrm{ft}$., open stony pasture, 08. 1910, Forrest 6357" (holotype E!).

= Peucedanum heterophyllum Franch., 1894, Bull. Annuel Soc. Philom. Paris (sér. 8) 6: 141, nom. illeg. (Art. 53.1), non Vis. (1836). 
$=$ Peucedanum franchetii $\mathrm{Wu}$ Zhengyi et $\mathrm{Pu}$ Fating, 1993, in Wang Wentsai (ed.), Vasc. Pl. Hengduan Mount.: 1349.

= Peucedanum paucipartitum Sheh Menglan 1997, in Wu Zhengyi (ed.), Fl. Yunnan. 7: 617, tab. 199, fig. 7-11.

Type: CHINA. "Yun-nan, in pratis calcareis montis Hee-chan-men, alt. 3000 m, 03. 10. 1884, Delavay 192" (lectotype P! - barcode P02272008, designated here; isolectotype P! barcode P02272009); "Yun-nan, prairies des hautes montagnes de Koua la po, à 3000 m. d'altit., 26. 08. 1884, Delavay 950" (syntypes P! - barcodes P02272010, P02272011).

Distribution in China: SW: Sichuan, Yunnan. Endemic.

Ligusticopsis involucrata (Franch.) Lavrova [1996, Mat. IX Moscow Conf. Phylogenet. Plant.: 78, comb. inval. (Art. 41.5)] ex Pimenov, comb. nov.

$\equiv$ Ligusticum involucratum Franch., 1894, Bull. Annuel Soc. Philom. Paris (sér. 8) 6: 136.

Type: CHINA. "Yunnan, Fentes de roches calcaires, au sud et près du col de Yen-tze-hay (Lan-kong) à $3200 \mathrm{~m}$ d'altit., 27. 08. 1886, Delavay 2330" (lectotype P! - barcode P03224970, designated by Leute, 1970: 492; isolectotypes K! barcode K000685171, P - barcodes P03224582, P03224971, P03224976); "Yun-nan, in fissuris rupium calcarearum ad cacumina montis Che-tchotze supra Tapin-tze. 10. 10. 1882, Delavay 448" (syntype P - barcodes P03224580, P03224974, P03224975); “Ad cacumina montis Ki-chan, prope Tali, alt. 2800 m, 10. 08. 1884, Delavay" (syntype $\mathrm{P}$ - barcode P03224972); "In pascuis ad cacuminal montis Hee-chan-men, alt. 3300 m,11. 09. 1885, Delavay 2022" (syntype P - barcode P03224581); "In rupibus calcareis montis Ma-eul-chan, alt. 2500 m, 09. 09. 1889, Delavay 3910" (syntype GH barcode GH00112220).

= Pleurospermum likiangense H. Wolff, 1929, Repert. Spec. Nov. Regni Veg. 27: 116.

$=$ Ligusticopsis likiangensis (H. Wolff) Lavrova et Kljuykov, 1994, Bot. Zhurn. (Leningrad) 79(10): 104.

$=$ Ligusticum likiangense $(\mathrm{H}$. Wolff) Pu Fating et M. F. Watson, 1994, Acta Phytotax. Sin. 42(6): 563.

Type: CHINA. "Yunnan, Yangtze watershed, prefectural district of Likiang, eastern slopes of Likiang Snow Range, $13500 \mathrm{ft}$., above Mahang Patze, 18. 08. 1922, Rock 5953A" (holotype US!).

= Ligusticum calophlebicum H. Wolff, 1930, Repert. Spec. Nov. Regni Veg. 27: 310.
= Pleurospermum calophlebicum (H. Wolff) M. Hiroe, 1958, Umbell. Asia 1: 122.

Type: CHINA. "Yunnan: Yangtze watershed, prefectural district of Likiang, eastern slopes of Likiang Snow Range, 9000-12000', 16. 08. 1922, Rock 5844" (syntypes E! - barcode E00002348, UC!, US - barcode US00127274); "Yunnan, Yangtze watershed; prefectural district of Likiang, eastern slopes of Likiang Snow Range, 11000' s.m., 19. 08. 1922, Rock 5912" (syntype); "Yunnan: Yangtze watershed; prefectural district of Likiang, eastern slopes of Likiang Snow Range, 9000' s.m., 22. 08. 1922, Rock 6056" (syntype).

- Peucedanum delavayi auct. non Franch.: Sheh Menglan, 1992, in Shan Renhwa \& Sheh Menglan (eds.), Fl. Reipubl. Popularis Sin. 55(3): 130, tab. 55, fig. 1-5.

Distribution in China: Tibet: Xizang A. R.; SW: Sichuan, Yunnan. Endemic.

Ligusticopsis longicalycia (Sheh Menglan) Pimenov et Kljuykov, 1999. Feddes Repert. 110(78): 484.

ESelinum longicalycium Sheh Menglan, 1992, J. P1. Resourc. Environ. 1(3): 1.

Type: CHINA. "Yunnan, Weixi, alt. 3600 m, under alpine conisilvae, 25. 10. 1960, Kunming Inst. Bot. Exped. 25257" (holotype KUN!).

Distribution in China: SW: Yunnan. Endemic.

Ligusticopsis modesta (Diels) Leute, 1969, Ann. Naturhist. Mus. Wien 73: 78, tab. 5, fig. 3.

इLigusticum modestum Diels, 1912, Notes Roy. Bot. Gard. Edinburgh 5: 289.

Type: CHINA. "[Yunnan] grassy openings in pine forests on the eastern flank of Lichiang Range. Lat. $27^{\circ} 20^{\prime}$ N. Alt. 10-11000 ft., 08. 1906, Forrest 2856" (lectotype E! - barcode E00265238, designated here; isolectotypes BM! - barcode BM000885419, P! - barcode P03224938).

Distribution in China: Tibet: Xizang A. R.; SW: Sichuan, Yunnan. Endemic.

Ligusticopsis multivittata (Franch.) Leute, 1969, Ann. Naturhist. Mus. Wien 73: 74, tab. 4, fig. 2.

इLigusticum multivittatum Franch., 1894, Bull. Annuel Soc. Philom. Paris (sér. 8) 6: 133.

Type: CHINA. "In collibus lapidosis ad montem Ma-eul-chan, alt. $3800 \mathrm{~m}$ [Côteaux rocalleux du Ma eul chan, à $3300 \mathrm{~m}$ d'alt.], 23. 10. 1889, Delavay" (holotype P! - barcode P03224923).

= Trachydium hispidum H. Wolff, 1930, Repert. Spec. Nov. Regni Veg. 27: 329, nom. inval. (Art. 53.1), non Franch. (1894). 
= Trachydium lichiangense Wu Zhengyi, 1984, Index Fl. Yunnan. 1: 929.

Type: CHINA. "Yunnan, Yangtze watershed, prefectural district of Likiang, eastern slopes of Likiang Snow range, 05-10. 1922, Rock 5353" (holotype US; isotype E!).

Distribution in China: SW: Sichuan, Yunnan. Endemic.

Ligusticopsis pseudodaucoides (Peng Hua et Wang Yinzheng) Pimenov et Kljuykov, 1999, Feddes Repert. 110(7-8): 485.

$\equiv$ Ligusticum pseudodaucoides Peng Hua et Wang Yinzheng, 1998, Novon 8: 50, fig. 1.

Type: CHINA. "Yunnan: Jingdong Yi Nationality Autonomous Country, heath on top of Mt. Maotou, Wulianshan Range, 2413'-11 N, 100'39'-46 E, 3306 m, 19. 11. 1996, Peng Hua 2579" (holotype KUN).

Distribution in China: SW: Yunnan. Endemic.

Distribution outside China: Endemic to Eastern Asia.

Ligusticopsis purpurascens Lavrova et Kljuykov, 1994, Bot. Zhurn. (Leningrad) 79(10): 106.

Type: CHINA. "Prov. Sze-Ch'uan, reg. bor.occid. Tsipula, ca $4000 \mathrm{~m}$ s.m., 05. 08. 1922, H. Smith 4197" (holotype GB!).

Distribution in China: SW: Sichuan. Endemic.

Ligusticopsis scapiformis (H. Wolff) Leute, 1969, Ann. Naturhist. Mus. Wien 73: 77, tab. 4, fig. 4.

三Ligusticum scapiforme H. Wolff, 1930. Repert. Spec. Nov. Regni Veg. 27: 308.

Type: CHINA. "Yunnan bor.-occid.: prope urbem Lidjang (Likiang) imprimis in monte Yulung-schan, 1914-16, collectores indigeni 3843" (syntypes W!, WU! - barcode WU0025132); "Yunnan: Yangtze watershed, prefectural district of Likiang, eastern slopes of Likiang Snow Range, 27. 06. 1922, Rock 4744" (syntypes E! - barcode E00002343, UC!, US).

= Ligusticum maxonianum H. Wolff, 1930, Repert. Spec. Nov. Regni Veg. 27: 316.

Type: CHINA. "Yunnan: Eastern slopes of Mount Dyinaloko, northern peak of the Likiang Snow Range, 14000' s.m., 08. 1923, Rock 10380" (lectotype E! - barcode E00002349, designated here; isolectotypes UC!, US! - barcode US00127287).

= Ligusticum pseudomodestum H. Wolff, 1930, Repert. Spec. Nov. Regni Veg. 27: 325.
Type: CHINA. "Tibet: Glacier Lake Camp, N $28^{\circ} 5^{\prime}$, E $100^{\circ} 45^{\prime}, 13000$ ft., Kingdon-Ward 4664” (holotype E! - barcode E00002396).

Distribution in China: Tibet: Xizang A. R.; SW: Sichuan, Yunnan. Endemic.

Ligusticopsis wallichiana (DC.) Pimenov et Kljuykov, 2001, Willdenowia 31(1): 122.

$\equiv$ Peucedanum wallichianum DC., 1830, Prodr. 4: 181 .

इSelinum tenuifolium Wall. ex C. B. Clarke, 1879, in Hooker f. (ed.), Fl. Brit. India 2: 700, nom. illeg. (Art. 53.1), non Salisb. (1796).

三Selinum wallichianum (DC.) Raizada et H. O. Saxena, 1966, Indian Forester 92(5): 323.

Type: INDIA. "In India orientalis ad Sirinagar, Wallich [579.1]" (syntypes E!, G-DC!, K!, K-WALLICH!, M). NEPAL. "In Nepaliae monte Emodo ad Gossain-Than, Wallich [579.2]" (lectotype G-DC!, designated by Watson, 1988: 380; isolectotypes BM, CAL, K!).

Distribution in China: Tibet: Xizang A. R., Qinghai; SW: Yunnan.

Distribution outside China: Myanmar, Bhutan, Nepal, India.

Ligusticopsis xizangensis (Pan Zehui et Sheh Menglan) Pimenov et Kljuykov, comb. nov.

$\equiv$ Ligusticum xizangense Pan Zehui et Sheh Menglan, 1992, Acta Phytotax. Sin. 30(3): 265, fig. 2.

Type: CHINA. "Xizang: Nyingchi, alt. 4500 m, among grasses, 10. 10. 1989, Yao Gan et al. 2409" (holotype NAS).

Distribution in China: Tibet: Xizang A. R. Endemic.

Ligusticum gongshanense Pu Fating, Li Rong et Li Heng, 2012, Nordic J. Bot. 30: 181.

Type: CHINA. "Yunnan Province, Gongshan Xian, Cikai Zheng, about 1.2 direct $\mathrm{km}$ by southeast of the Heipu Pass tunnel on the new road from Gongshan to the Dulong Jiang valley, east side of Gaoligong Shan, wet meadow with stream, some scattered rocks, growing along stream, $27^{\circ} 45^{\prime} 41.7^{\prime} ' \mathrm{~N}, 98^{\circ} 27^{\prime} 2.3$ 'E, $3350 \mathrm{~m}$ a.s.1., 13. 08. 2006, Gaoligong Shan Biodiversity Survey 32097" (holotype KUN; isotype CDBI).

Distribution in China: S: Yunnan. Endemic.

Note: The species can belong to Ligusticopsis Leute.

Ligusticum kiangsiense H. Wolff, 1930, Repert. Spec. Nov. Regni Veg. 27: 326. 
Type: CHINA. "Nord-Kiangsi, Du BoisReymond 733" (holotype B?).

Distribution in China: Centr.: Jiangsu, Anhui, Jiangxi. Endemic.

Ligusticum kulingense H. Wolff, 1930, Repert. Spec. Nov. Regni Veg. 27: 314.

Type: CHINA. "Nord-Kiangsi: Kuling Suschangebirge, $1100 \mathrm{~m}, 07$. 1915, Du BoisReymond" (holotype B?).

Distribution in China: Centr.: Jiangxi. Endemic.

Ligusticum littledalei Fedde ex H. Wolff, 1930, Repert. Spec. Nov. Regni Veg. 27: 327.

Type: CHINA. "Central-Tibet, Littledale".

Distribution in China: Tibet: Xizang A. R. Endemic.

Ligusticum yanyuanense Pu Fating, 1991, Acta Phytotax. Sin. 29(6): 526, fig. 2.

Type: CHINA. "Sichuan: Yanyuan, alt. 3800 m, in bush-woods and grassland, 21. 07. 1983, Exped. Qinghai-Xizang 83-12179" (holotype PE; isotype KUN).

Distribution in China: SW: Sichuan. Endemic.

Ligusticum yunnanense Pu Fating, 1991, Acta Phytotax. Sin. 29(6): 543, fig. 10.

Type: CHINA. "Yunnan, 1938, Liou Tchenngo 2576" (holotype KUN).

Distribution in China: SW: Yunnan. Endemic.

Ligusticum yushuense Pan Jintang, 1999, Fl. Qinghaica 2: 433, 400, tab. 67, fig. 8-14.

Type: CHINA. "Qinghai: Yushu, ad margines sylvarum alt. $3550 \mathrm{~m}, 26.07 .1980$, Z. D. Wei 21892" (holotype HNWP).

Distribution in China: Tibet: Qinghai. Endemic.

Lithosciadium kamelinii (V. M. Vinogr.) Pimenov, 1996, in Gubanov, Konsp. Fl. Vneshnei Mongolii (Sosud. Rast.): 79.

$\equiv$ Cnidium kamelinii V. M. Vinogr., 1988, Novosti Sist. Vyssh. Rast. 25: 122.

Type: MONGOLIA. "Altai Mongolicus, systema fl. Bulgan-Gol, in fluxu superiore angustiarum fl. Uljastyjn-Gol, ad affluxiones sinistras, 09. 07. 1984, Kamelin, Darijimaa 306" (holotype LE!).

Distribution in China: NW: Xinjiang Weiwuer A. R.

Distribution outside China: Mongolia.
Lomatocarpa albomarginata (Schrenk) Pimenov et Lavrova, 1987, Bot. Zhurn. (Leningrad) 72(1): 35 .

$\equiv$ Neogaya simplex (L.) Meisn. var. albomarginata Schrenk, 1842, Enum. Pl. Nov. 2: 41.

$\equiv$ Pachypleurum albomarginatum (Schrenk) Rupr., 1869, Mém. Acad. Imp. Sci. Saint-Petersbourg (ser. 7) 14(4) (Sert. Tiansch.): 49.

$\equiv$ Neogaya albomarginata (Schrenk) O. et B. Fedtsch., 1909, Consp. Fl. Turkest. 3: 97.

$\equiv$ Alposelinum albomarginatum (Schrenk) Pimenov, 1982, Byull. Moskovsk. Obšč. Isp. Prir. Otd. Biol. 87, 1: 113.

Type: KAZAKHSTAN. "Songarei, Dshatyk, 22. 07. 1841, Schrenk 851" (holotype LE!).

= Ligusticum wolffianum Fedde ex H. Wolff, 1930, Repert. Spec. Nov. Regni Veg. 27: 318.

Type: AFGHANISTAN. "Pamir [Pamir Boundary Commission], in nullahs alongside running water, 14000-15000 ft.,1895, Alcock 17712" (lectotype BM! - barcode BM000826930, designated here; isolectotype DD!).

= Pachypleurum aemulans Korovin, 1962, in Pavlov (ed.), Fl. Kazakhst. 6: 309, nom. inval. (Art. 39.1), descr. ross.

= Cortia schmidii Nasir, 1972, in Nasir \& Ali (eds.), Fl. W Pakistan 20: 115, fig. 34 (A-C).

$=$ Ligusticum schmidii (Nasir) Farille et S. B. Malla, 1985, Candollea 40(2): 551.

Type: PAKISTAN. "Chitral, upper Yarkhun Vy, Schmid 2365" (holotype RAW).

- Neogaya simplex auct. non Meisn.: O. et B. Fedtsch., 1911, Beih. Bot. Centralbl. 28(2): 42.

- Pachypleurum alpinum auct. non Ledeb.: Schischk., 1950, in Schischkin (ed.), Fl. URSS 16: 579, p.p.

Distribution in China: NW: Xinjiang Weiwuer A. R.

Distribution outside China: Pakistan, Kazakhstan, Kirghizia, Tajikistan, Uzbekistan, Afghanistan.

Meeboldia achilleifolia (DC.) P. K. Mukh. et Constance, 1991, Edinburgh J. Bot. 48(1): 44.

EPtychotis achilleifolia DC., 1830, Prodr. 4: 109.

$\equiv$ Pimpinella achilleifolia (DC.) C. B. Clarke, 1879, in Hooker f. (ed.), Fl. Brit. India 2: 684.

三Vicatia achilleifolia (DC.) P. K. Mukh. [1982, Candollea 37(2): 560, nom. inval. (Art. 41.5), without basionym ref.] 1983, Bull. Bot. Surv. India 24(1-4): 43.

$\equiv$ Tongoloa achilleifolia (DC.) Pimenov et Kljuykov, 1991, Feddes Repert. 102(5-6): 383. 
Type: INDIA. "In Napalem, Kamaon, 1821, Wallich [568.1]" (lectotype G-DC!, designated by Watson, 1996: 137; isolectotypes BM! barcodes BM000049863, BM000049866, C! barcode C10008514, CAL, CGE!, E! - barcodes E00000237, E00000238, G - barcode G00367118, K! - barcode K000697384, K-WALLICH! - barcode K000697385, LE!, M! - barcode M-0172952).

= Meeboldia selinoides H. Wolff, 1924, Repert. Spec. Nov. Regni Veg. 19: 313.

Type: INDIA. "Himalaya: in valle Naini, 6500' s.m., Meebold 3402" (holotype BM - barcode BM00062295).

Distribution in China: Tibet: Xizang A. R.; SW: Yunnan.

Distribution outside China: Nepal, India.

Melanosciadium bipinnatum (Shan Renhwa et Pu Fating) Pimenov et Kljuykov, 2006, Feddes Repert. 117(7-8): 471, fig. 2, 3, 13.

$\equiv$ Vicatia bipinnata Shan Renhwa et Pu Fating, 1986, Acta Phytotax. Sin. 24(4): 313, fig. 9.

$\equiv$ Sinodielsia bipinnata (Shan Renhwa et $\mathrm{Pu}$ Fating) Pimenov et Kljuykov, 1991 Feddes Repert. 102(5-6): 383.

Type: CHINA. "Sichuan, Maerkang County, on the hills behind the hospital, alt. $2700 \mathrm{~m}, 27.09$. 1964, Yue Jinsan, Song Guojie, Zhuang Tide 6405" (holotype NAS!; isotype NAS).

= Pimpinella rhomboidea Diels var. tenuiloba Shan Renhwa et Pu Fating, 1989, Acta Phytotax. Sin. 27(1): 63, tab. 2.

Type: CHINA. "Sichuan Province, Lixian, Putouxiang, Suoluogou, alt. $3400 \mathrm{~m}$, in alpine meadows, 03. 09. 1983, Lixian Medic. Herb. Exped. 20800" (holotype CDBI!).

Distribution in China: SW: Sichuan, Yunnan. Endemic.

Melanosciadium genuflexum Pimenov et Kljuykov, 2006, Feddes Repert. 117(7-8): 473, fig. 4-7, 14, 15.

Type: CHINA. "Yunnan, Midu, 10. 11. 1961, Yang Tingsheng 610337" (holotype KUN!).

Distribution in China: SW: Sichuan, Yunnan. Endemic.

Melanosciadium pimpinelloideum $\mathrm{H}$. Boissieu, 1902, Bull. Herb. Boissier (2 sér.) 2: 804.

$\equiv$ Pimpinella pimpinelloidea (H. Boissieu) M. Hiroe, 1979, Umbelliferae World: 835, "pimpinelloideum".

Type: CHINA. "Su-tschuen à Tchen-Kéou-Tin, Farges 469" (lectotype P! - barcode P00752796, designated here; isolectotypes E!, K, P! - barcodes P00752797, P00752798, P00752799, P00752800, PE!).

= Angelica involucellata Diels, 1900, Bot. Jahrb. Syst. 29 (Fl. Centr. China): 499.

Type: CHINA. "Setchuen, Nan ch'uan: Yachihpa, Wald, 16. 08. 1891, von Rosthorn 484" (lectotype O!, designated here); "Setchuen, Nan ch'uan: T'ai ho-tung, 13. 09. 1891, von Rosthorn 890" (syntype O!).

Distribution in China: Centr.: Hubei, Hunan; SW: Sichuan, Guizhou, Yunnan. Endemic.

Nothosmyrnium japonicum Miq., 1867, Ann. Mus. Bot. Lugduno-Batavi 3: 58.

Type: JAPAN. "Japonia, Siebold" (lectotype Lbarcode L01754259, designated in L; isolectotypes L - barcodes L0175429, L0175431); "Japonia. Bürger" (syntypes L - barcodes L0175427, L0175430); "Japonia. Mohnike" (syntype L barcode L0175426).

= Macrochlaena glaucocarpa Hand.-Mazz., 1933, Symb. Sin. 7(3): 720, fig. 22 (1-3).

Type: CHINA. "SW-Hunan, Hochstauden Fluren im wtp Laubwaldes des Yun-schan bei Wukang, Tonschiefer, 1200-1350 m, 09-13. 08. 1918, Handel-Mazzetti 12422" (lectotype WU!, designated here; isolectotypes C!, E! - barcode E00000550, W!, WU!).

Distribution in China: NW: Gansu; N: Shaanxi, Hebei; Centr.: Henan, Hubei, Jiangsu, Anhui, Hunan, Jiangxi, Zhejiang, Fujiang; SW: Sichuan, Guizhou; S: Guangxi Zhuang A. R., Guangdong Endemic.

Nothosmyrnium japonicum Miq. var. sutchuenense H. Boissieu, 1909, Bull. Soc. Bot. France 56: 349.

Type: CHINA. "Su-tschuen à Tchen-KéouTin, Farges" (lectotype P! - barcode P00752794, designated here; isolectotypes $\mathrm{B}$ !, $\mathrm{K}$ !).

Distribution in China: SW: Sichuan, Yunnan; S: Guangxi Zhuang A. R. Endemic.

Nothosmyrnium xizangense Shan Renhwa et Wang Tiehseng, 1980, Acta Phytotax. Sin. 18(3): 375.

Type: CHINA. "Xizang: Mailing Xian, alt. 3200 $\mathrm{m}$, in montibus faucibus et in sylvis crescens, 26. 08. 1974, Qinghai-Xizang Exped. 74-1985" (holotype $\mathrm{PE})$.

Distribution in China: Tibet: Xizang A. R.; SW: Sichuan. Endemic. 
Nothosmyrnium xizangense Shan Renhwa et Wang Tiehseng var. simpliciorum Shan Renhwa et Wang Tiehseng, 1980, Acta Phytotax. Sin. 18(3): 376.

Type: CHINA. "Xizang: Mailing Xian, alt. 3400 $\mathrm{m}$, in montanis clivis et in sylvis crescens, 13. 09. 1974, Qinghai-Xizang Exped. 4695” (holotype PE).

Distribution in China: Tibet: Xizang A. R. Endemic.

Notopterygium tenuifolium Sheh Menglan et $\mathrm{Pu}$ Fating [1997, J. P1. Resourc. Environ. 6(2): 41, fig. 1, nom. inval.] 2000, Acta Phytotax. Sin. 38(5): 435.

Type: CHINA. "Sichuan: Litang to Yajiang on the way Karazi Shan, alt. 4300 m, among grass places in mountain valley, 09. 1984, Pu Fating, Yao Gan 697, 623" (holotype NAS).

Distribution in China: SW: Sichuan, Endemic.

Note: A little-known species of unclear generic placement.

Oenanthe benghalensis (DC.) Miq., 1870, Ill. F1. Arch. Ind. 1: 42. 140.

E Dasyloma benghalense DC., 1830, Prodr. 4:

三 Seseli bengalense Roxb., 1814, Hort. Beng.: 22, nom. inval.; 1832, Fl. Ind. (ed. 2) 2: 94.

Type: INDIA. "In Bengali orientalis prov. Sillet, De Silva [582]" (lectotype G-DC, designated here; isolectotypes K! - barcodes K0006813219, K000681323, K000681324, K-WALLICH! barcodes K000681320,000681321, K0006811325).

= Dasyloma glaucum DC., 1830, Prodr. 4: 140.

Type: INDIA. "Ex Bengalo verosimiliter ortum, in horto Calcuttensi cultum, Blakeworth [587]" (lectotype G-DC!, designated here; isolectotypes $\mathrm{K}$ !, K-WALLICH!).

Distribution in China: Centr.: Zhejiang; SW: Yunnan; S: Taiwan, Guangxi Zhuang A.R., Guangdong, Hainan Dao.

Distribution outside China: Vietnam, Thailand, Bangladesh, India.

\section{7.}

Oenanthe caudata C. Norman, 1929, J. Bot. 67:

Type: CHINA. "Szechuan, 1885-1889, Henry 7152” (lectotype BM! - barcode BM000944751, designated here; isolectotypes E! - barcodes E00000560, E00000561, E00000569, K! - barcode K000681339, US).

$=$ Oenanthe dielsii $\mathrm{H}$. Boissieu var. stenophylla H. Boissieu, 1906, Bull. Acad. Int. Geogr. Bot. 16: 185.
$=$ Oenanthe dielsii $\mathrm{H}$. Boissieu subsp. stenophylla (H. Boissieu) Wu Zhengyi et Pu Fating, 1993, in Wang Wentsai (ed.), Vasc. P1. Hengduan Mount.: 1333.

= Oenanthe thomsonii C. B. Clarke subsp. stenophylla (H. Boissieu) Pu Fating, 1998, Novon 8(1): 71.

Type: CHINA. "Sichuan: Cheng kou [Su-Tchuen oriental, district de Tchen-Kéou-Tin], Farges [79]" (lectotype P! - barcode P00835221, designated here; isolectotypes $\mathrm{K}$ ! - barcodes K000681331, K000681333, P! - barcodes P00835219, P00835220, P02518583, P02518791).

Distribution in China: Centr.: Hubei; SW: Sichuan. Endemic.

Oenanthe dielsii H. Boissieu, 1906, Bull. Acad. Int. Geogr. Bot. 15: 184.

Type: CHINA. "Yunnan-sen, district PinFa, près la chute d'eau, 08. 1902, Cavalerie 240" (lectotype P!, designated here; isolectotypes E! barcode E00002341, P! - barcode P00835217).

Distribution in China: Tibet: Xizang A. R.; N: Shaanxi; Centr.: Henan, Hubei, Jiangxi, Zhejiang, Fujiang; SW: Sichuan, Guizhou, Yunnan; S: Guangxi Zhuang A. R., Guangdong.

Distribution outside China: Vietnam.

Oenanthe hookeri C. B. Clarke, 1879, in Hooker f. (ed.), Fl. Brit. India 2: 697.

Type: BANGLADESH. "Khasia Mts., alt. 5000-6000 ft. 05. 07. 1850, Hooker and Thomson [1489]" (lectotype K! - barcode K000681313, designated by Watson, 1998: 375; isolectotypes E! - barcode E00265212, G! - barcode G00367115, K! - barcodes K000681315, K000681316, M barcode M0172909, NY - barcode NY00406087, P! - barcodes P02518781, P02518782, P02518783, P02518784, STU).

Distribution in China: Tibet: Xizang A. R.; SW: Sichuan, Guizhou, Yunnan.

Distribution outside China: Bhutan, Nepal, India.

Oenanthe javanica (Blume) DC., 1830, Prodr. 4: 138 .

三Sium javanicum Blume, 1826, Bijdr. Fl. Ned. Ind. 15: 881.

EFalcaria javanica (Blume) DC., 1830, Prodr. 4: 110 .

三 Dasyloma javanicum (Blume) Miq., 1855, Fl. Ned. Ind. 1(1): 741.

$\equiv$ Oenanthe stolonifera DC. var. javanica (Blume) Kuntze, 1891, Revis. Gen. P1. 1: 269. 
Type: INDONESIA. "Java, Zippelius" (syntypes L! - barcodes L0008366, L0008367, L0008368); "In paludosis Javae ubi dicitur Tespong awa Barat, Tespong [Java Tespong]. Blume" (lectotype L! barcode L0008364, designated here; isolectotypes K!, L! - barcodes L0008363, L0008365, L0008370, L0008372, NY).

= Sium laciniatum Blume, 1826, Bijdr. Fl. Ned. Ind. 15: 881.

$=$ Falcaria laciniata (Blume) DC., 1830, Prodr. 4: 110 .

= Oenanthe laciniata (Blume) Zoll., 1854, Syst. Verz. 2: 139 .

= Dasyloma laciniatum (Blume) Miq., 1855, Fl. Ned. Ind. 1(1): 741.

Type: INDONESIA. "In paludosis Tjisere provinciae Bataviae [Java], Blume" (lectotype L! barcode L0008373, designated here; isolectotypes $\mathrm{K}$ !, L!).

$=$ Oenanthe stolonifera DC., 1830, Prodr. 4: 138.

$=$ Oenanthe javanica (Blume) DC. subsp. stolonifera (DC.) Murata, 1973, Acta Phytotax. Geobot. 25(4-6): 105, tab. 2, fig. 3.

Type: BANGLADESH. "Silhet Bengali orientale [Cherrapungee et Silhet], 08. 1830, De Silva [585] (lectotype G-DC!, designated here; isolectotypes CAL, E! - barcodes E00041853, E00041854, K! barcodes K001111342, K001111343, K001111345, K-WALLICH! - barcode K0006811329, LE!).

= Dasyloma latifolium Lindl., 1835, in Royle, Ill. Bot. Himal. Mts.: 232.

Type: INDIA. "[NW India] Choor, Royle" (lectotype K!, designated here; isolectotype LE!).

$=$ Oenanthe corticata Edgew., 1846, Trans. Linn. Soc. London 20(1): 53.

= Dasyloma corticatum (Edgew.) Miq., 1855, Fl. Ned. Ind. 1(1): 742.

$=$ Oenanthe stolonifera DC. var. corticata (Edgew.) C. B. Clarke, 1879, in Hooker f. (ed.), Fl. Brit. India 2: 696.

Type: INDIA. "In India Boreali-Occidentali, rivulis stagnisque prov. Sirhind, Raduur, Edgeworth 169" (holotype K!).

$=$ Cyssopetalum javanicum Zoll. ex Turcz., 1849, Bull. Soc. Imp. Naturalistes Moscou 22(2): 26.

Type: INDONESIA. "Java [Plantae javanicae a cl. Zollinger lectae] Zollinger 2588" (syntypes KW, M).

= Dasyloma subbipinnatum Miq., 1867, Ann. Mus. Bot. Lugduno-Batavi 3: 59.

= Oenanthe subbipinnata (Miq.) Drude, 1898, in Engler \& Prantl (eds.), Nat. Pflanzenfam. 3(8): 204.

Type: JAPAN. "Probabiliter in Nippon, Keiske, Textor, Mohnike".
= Dasyloma japonicum Miq., 1867, Ann. Mus. Bot. Lugduno-Batavi 3: 59.

$=$ Oenanthe stolonifera DC. var. japonica (Miq.) Maxim. ex Franch. et Sav., 1875, Enum. P1. Jap. 1: 185.

= Oenanthe japonica (Miq.) Drude, 1898, in Engler \& Prantl (eds.), Nat. Pflanzenfam. 3(8): 204.

$=$ Oenanthe javanica (Blume) DC. var. japonica (Miq.) Honda, 1939, Nom. Pl. Japon.: 511.

Type: JAPAN. "Prope $\mathrm{Na}[n] g a s a k i$, Oldham 296".

= Oenanthe stolonifera DC. var. khasiana C. B. Clarke, 1879, in Hooker f. (ed.), Fl. Brit. India 2: 696.

Type: INDIA. "Khasia Mts., near Moflong, alt. $6000 \mathrm{ft}$., Hooker, Thomson" (holotype CAL).

= Oenanthe schlechteri H. Wolff, 1905, in Schum. \& Laut., Nachtr. Fl. deut. Schutzgeb. Sudsee: 333, fig. 14.

Type: PAPUA-NEW GUINEA. "New Guinea, Schumann River, Schlechter 13821".

= Oenanthe decumbens Koso-Pol., 1916, Bull. Soc. Imp. Naturalistes Moscou, s.n. 29: 130, fig. 7 , nom. illeg., non Sium decumbens Thunb.

= Oenanthe kudoi Suzuki et Yamam., 1932, Trans. Nat. Hist. Soc. Formosa 22: 408.

Type: CHINA. "[Taiwan] In monte Taihei, 17. 07. 1929, Suzuki 1066".

= Oenanthe normanii F.P.Metcalf, 1934, Lingnan Sci. J. 13(3): 517, tab. 57.

Type: CHINA. "Near Mong-so-lan, Hinghuo, Fukuen [Hingwa and vicinity], 14-26. 07. 1926, Lin Pi 6039" (holotype Fukuen Un.; isotypes A barcode A00077103, Lingnan Univ.).

$=$ Oenanthe stolonifera DC. f. elongata F. P. Metcalf, 1934, Lingnan Sci. J. 13(3): 518.

Type: CHINA. "[Guandong], Sai Sha, Sz Ooi, $500 \mathrm{ft}$., fruit orchard, at base of tree [Kwong Tung Province, North River Region]. 04. 1918, Peng 2401" (holotype A - barcode A00135100).

Distribution in China: NW: Gansu; Tibet: Xizang A. R.; N: Neimenggu A. R., Ningxia Huizu A. R., Shaanxi, Shanxi, Hebei, Shandong; NE: Heilongjiang, Jilin, Liaoning; Centr.: Henan, Jiangsu, Anhui, Hubei, Hunan, Jiangxi, Zhejiang, Fujiang; SW: Sichuan, Guizhou, Yunnan; S: Taiwan, Guangxi Zhuang A.R., Guangdong, Hainan Dao.

Distribution outside China: Russia, Japan, Korea, Philippines, Papua-New Guinea, Indonesia, Malaysia, Vietnam, Laos, Thailand, Myanmar, Bangladesh, Bhutan, Nepal, India, Pakistan, Afghanistan, Australia and Oceania. 
Oenanthe linearis Wall ex DC., 1830, Prodr. 4: 138.

$\equiv$ Oenanthe javanica (Blume) DC. subsp. linearis (Wall. ex DC.) Murata, 1973, Acta Phytotax. Geobot. 25(4-6): 103, tab. 2, fig. 4.

Type: NEPAL. "In agris orizaceis vallis Nepaliae [Silhet], 05.1821, Wallich [586]" (lectotype G-DC!, designated here; isolectotypes $\mathrm{BM}, \mathrm{C}$ ! - barcode C10008499, E! - barcodes E00002362, E00002363, K! - barcodes K000681317, K000681318, K-WALLICH! - barcodes K001111340, K001111341，K001111347， LE!，P - barcode P02518599).

= Oenanthe rivularis Dunn, 1903, J. Linn. Soc. Bot. 35: 496.

$=$ Oenanthe linearis Wall. ex DC. subsp. rivularis (Dunn) Wu Zhengyi et Pu Fating, 1993, in Wang Wentsai (ed.), Vasc. Pl. Hengduan Mount.: 1332.

Type: CHINA. "Yunnan, Mengtze, in moist places, often in small rills, 5000'. Henry 10822" (lectotype $\mathrm{K}$ !, designated here; isolectotypes E! barcode E00002342, MO - barcode MO-345461, NY - barcode NY 00406085, PE!, US).

= Oenanthe sinensis Dunn, 1903, J. Linn. Soc. Bot. 35: 496.

$=$ Oenanthe linearis Wall ex DC. subsp. sinensis (Dunn) Wu Zhengyi et Pu Fating, 1993, in Wang Wentsai (ed.), Vasc. Pl. Hengduan Mount.: 1333.

Type: CHINA. "Hupeh: Ichang, ricefields,05.1888, Henry 1663, 4089" (lectotype $\mathrm{K}$ ! - barcode K000681337, designated here; isolectotypes $\mathrm{E}$ ! - barcode E00002367, $\mathrm{K}$ - barcode K000681334, K000681336, LE!, P! - barcodes P02284808, P025118581); "Szechuen: Min River, Faber 874" (syntype K! - barcode K000681335).

Distribution in China: Tibet: Xizang A. R.; N: Shandong; Centr.: Henan, Jiangsu, Anhui, Hubei, Hunan, Jiangxi, Zhejiang, Fujiang; SW: Sichuan, Guizhou, Yunnan; S: Taiwan, Guangxi Zhuang A. R.

Distribution outside China: Japan, Indonesia, Vietnam, Myanmar, Nepal, India.

Oenanthe pterocaulon Liu Tangshui, Chao Chuangying et Chuang Tsaniang, 1961, Quart. J. Taiwan Mus. 14(1-2): 31.

Type: CHINA. "[Taiwan] Tapinlin, Taipei Hsien, 05. 07. 1957, M. T .Kao K3579” (holotype TAI).

Distribution in China: S: Taiwan. Endemic.

Oenanthe rosthornii Diels, 1900, Bot. Jahrb. Syst. 29 (3-4) (Fl. Centr. China): 498. $\equiv$ Oenanthe javanica (Blume) DC. subsp. rosthornii (Diels) Pu Fating, 1998, Novon 8(1): 70.

Type: CHINA. "Setchuen. Nan ch'uan: Mahu tzi wan. 20. 09. 1891, von Rosthorn 979" (lectotype O!, designated here); "Setchuen, Nan ch'uan: Shan tzu p'ing. 06. 08. 1891, von Rosthorn 307' (syntype O!)

Note: Pu Fating (1998: 70) proposed to designate a neotype (Guizhou: Ping-fa, 21. 08. 1902, Cavalerie 176 (if so, an isoneotype kept in K! - barcode K000681338). I found the valid type specimens in Oslo herbarium; so the designation of neotype is not needed.

= Oenanthe alatinervis Qian Yiyong, 1989, Guihaia 9(2): 117, fig. 1-5.

Type: CHINA. "Yunnan; Simao, 1400 m alt., in paludidus, 20. 08. 1987, Qian Yiyong 1410" (holotype Simao).

Distribution in China: Centr.: Hubei, Jiangsu, Hunan, Jiangxi, Fujiang; SW: Sichuan, Guizhou, Yunnan; S: Taiwan, Guangxi Zhuang A. R., Guangdong.

\section{Distribution outside China: Vietnam.}

Oenanthe thomsonii C. B. Clarke, 1879, in Hooker f. (ed.), Fl. Brit. India 2: 697.

Type:INDIA. "KhasiaMts., Churra, RegioTemp., alt. 5-6000 ft.,12. 06. 1850, Hooker, Thomson 585" (lectotype K! - barcode K000681310, designated by Watson, 1998: 375; isolectotypes G! - barcode G00367073, K - barcode K000681306, MH!, NY, P! - barcodes P02518658, P03518661, P03518662, S - barcodes S13-13577, S13-13579); "Sikkim, alt. 5000-8000 ft.,1857, Thomson" (syntypes E, K!, NYbarcode NY0046089).

Distribution in China: Tibet: Xizang A. R.; Centr.: Hubei, Hunan, Jiangxi; SW: Sichuan, Guizhou, Yunnan; S: Guandong.

Distribution outside China: Vietnam, Myanmar, Bhutan, Nepal, India.

Note: C. Norman in $\mathrm{K}$ determined many gatherings from China as Oenanthe caudata.

Oreocome limprichtii (H. Wolff) Pimenov et Kljuykov, 2001, Willdenowia 31(1): 118.

$\equiv$ Ligusticum limprichtii H. Wolff, 1922, Repert. Spec. Nov. Regni Veg. Beih. 12 (Limpr. Bot. Reise Hochgeb. China Ost-Tibet): 452.

Type: CHINA. "Yunnan: Tali fu, Tsang shan, 3000 m, Limpricht 1053" (holotype ?).

= Pleurospermum glaucescens H. Wolff, 1929, Repert. Spec. Nov. Regni Veg. 27: 114.

= Ligusticopsis glaucescens $(\mathrm{H}$. Wolff) Lavrova et Kljuykov, 1994, Bot. Zhurn. (St. Perersburg) 79(10): 105. 
Type: CHINA. "Yunnan, Yangtze watershed, prefectural district of Likiang, eastern slopes of Likiang Snow Range, Lu Kago, alpine meadow, 12000 ft., 16. 06. 1922, Rock 4516" (lectotype US!, designated by Pimenov et al., 2001: 120); "Yunnan, Yangtze watershed, prefectural district of Likiang, eastern slopes of Likiang Snow Range, 1922, Rock 4481" (syntype E! - barcode E00000157).

= Ligusticum kingdon-wardii H. Wolff, 1930, Repert. Spec. Nov. Regni Veg. 27: 306.

Type: CHINA. "Yunnan, A-tun-tsi, 13000', screes, 01. 08. 1913, Kingdon-Ward 925" (lectotype E! - barcode E00002397, designated here); "Mountains in NE of the Yangtze, Lat. 27'45', 10000', Forrest 10926" (syntype E! - barcode E00002398).

- Ligusticum sinense auct. non Oliv.: Hand.Mazz., 1933, Symb. Sin. 7(3): 723, coll. number 7824.

- Ligusticum discolor auct. non Ledeb.: Leute, 1970, Ann. Naturhist. Mus. Wien 74: 487, p.p., quoad pl. ex NW Yunnan.

Distribution in China: SW: Sichuan, Guizhou, Yunnan. Endemic.

Oreocome striata (DC.) Pimenov et Kljuykov, 2001, Willdenowia 31(1): 115.

$\equiv$ Ligusticum striatum DC., 1830, Prodr. 4: 158.

$\equiv$ Selinum striatum (DC.) Benth. ex C. B. Clarke, 1879, in Hook. f. (ed.), Fl. Brit. Ind. 2(6): 699.

$\equiv$ Ligusticum wallichii Franch., 1894, Bull.

Annuel Soc. Philom. Paris (sér. 8) 6: 136, quoad nomen, excl. pl.

$\equiv$ Cortia striata (DC.) Leute, 1969, Ann. Naturhist. Mus. Wien 73: 85.

Type: NEPAL. "Argos oryzaceos vallis Nepauliae magnae, 1821, Wallich [583]" (lectotype G-DC - barcode G00367289, designated by Watson, 1998: 380; isolectotypes BM! - barcode BM000049868, CAL, E! - barcode E00002353, G, K! - barcode, K-WALLICH! - barcodes K000685168, K000685169, LE!).

= Peucedanum dissectum DC., 1830, Prodr. 4: 178, p.p., nom. illeg. (Art. 53.1), non Ledeb. (1829).

$=$ Selinum dissectum (DC.) Wall. ex C. B. Clarke, 1879, in Hooker f. (ed.), Fl. Brit. India 2: 701, p.p.

Type: NEPAL. "In Emodo seu Himalaya Nepalensi ad Gossain-Than, 27. 10. 1821, Wallich [581]" (lectotype G-DC, designated by Watson, 1998: 379; isolectotypes CAL, CGE, K!, K-WALLICH!).

= Pimpinella bengalensis $\mathrm{H}$. Wolff, 1921, Repert. Spec. Nov. Regni Veg. 17: 170.
Type: INDIA. "Bengalia orientalis sine loco indic., Griffith 2615/1" (lectotype K, designated here; isolectotypes CAL, LE!, W!).

= Ligusticum gyirongense Shan Renhwa et Chang Hotseng, 1986, Acta Phytotax. Sin. 24(4): 315, fig. 11.

Type: CHINA. "Xizang, Gyirong, 04. 11. 1967, Jiang, Zhao 345" (holotype PE!).

Distribution in China: NW: Xinjiang Weiwuer A. R.; Tibet: Xizang A. R.; SW: Yunnan.

Distribution outside China: Vietnam, Thailand, Myanmar, Bhutan, Nepal, India.

Oreocomopsis dochenensis (W. W. Sm.) Pimenov \& Kljuykov, comb. nov.

$\equiv$ Pleurospermum dochenense W. W. Sm., 1914, Rec. Bot. Surv. India 6: 100.

Type: CHINA. "Tibet: Dochen plain (near Yantze?), 13. 08. 1907, Stewart 155" (lectotype E! barcode E00265239, designated here; isolectotype K! - barcode K000685339).

= Pleurospermum aromaticum W. W. Sm., 1915, Notes Roy. Bot. Gard. Edinburgh 8: 341.

$=$ Oreocomopsis aromatica $(\mathrm{W} . \mathrm{W} . \mathrm{Sm}$.) Pimenov et Kljuykov, 2000, Feddes Repert. 111(7-8): 519.

Type: CHINA. "Yunnan, West China, western flank of Lichiang Range. $27^{\circ} 30^{\prime}$ N. Ledges of cliffs and rocky alpine pasture 12000-13000 ft., 07. 1910, Forrest 6188" (lectotype E! - barcodes E00000159, E00000169, designated here; isolectotypes BM! barcode BN000944695, K!, PE!).

$=$ Ostericum scaberulum (Franch.) Yuan Changchi et Shan Renhwa var. longiinvolucellatum $\mathrm{Wu}$ Zhengyi et Pu Fating [in Wang Wentsai (ed.), 1993, Vasc. Pl. Hengduan Mts.: 1347, nom. inval (Art. 39.1), descr. chin.], 1998, Novon, 8(1): 70.

Type: CHINA. "Yunnan, Bai-mar-shan, A-tuntze, alt. $3400 \mathrm{~m}$, mountain slope [Degen, Baima Snow Mount, among shrubs], 09. 1935, C. W. Wang 69408" (holotype KUN; isotype NAS!).

Distribution in China: Tibet: Xizang A. R.; SW: Sichuan, Yunnan. Endemic.

Oreocomopsis xizangensis Pimenov et Kljuykov, 1996, Acta Phytotax. Sin. 34(1): 3, fig. 1, 3.

Type: CHINA. "Xizang, China: alt. 5100-5300 m s.l., declivum septentrionale montis Qomolamgma, prope monasterium Rongbuk, declivum shistosum moraenae recentis, 10. 09. 1991, Tischkov" (holotype MW!; isotype E!).

Distribution in China: Tibet: Xizang A. R. Endemic. 
Oreomyrrhis involucrata Hayata, 1911, J. Coll.

Sci. Univ. Tokyo 30(1): 128.

$\equiv$ Chaerophyllum involucratum (Hayata) Chung

Kuofang, 2007, Syst. Bot. 32(3): 678.

Type: CHINA. "[Taiwan] In monte Morrison [Yushan], ad 12000 ped. alt., 20. 10. 1906, Kawakami, Mori 2249" (lectotype TI, designated by Chen Chihhsiung, Wang Jeenche, 2001: 306; isolectotype TAIF); "[Taiwan] Im monte Morrison, 11. 1905, Nagasawa 756" (syntype TI!).

= Oreomyrrhis gracilis Masam., 1931, J. Soc.

Trop. Agric. 3(1): 20.

$=$ Oreomyrrhis involucrata Hayata var. gracilis Masam., 1938, Trans. Nat. Hist. Soc. Taiwan 28: 139.

Type: CHINA. "Taiwan, Sikayotai-zan, ca. 3300 m, 07. 07. 1930, Masamune 1239" (holotype TAI).

=Oreomyrrhis involucrata Hayata var. pubescens Masam., 1938, Trans. Nat. Hist. Soc. Taiwan 28: 138.

Type: CHINA. "Taiwan: Tyosenzan Be Sipau, 2800 m, 28. 07. 1936, Suzuki 16258” (holotype TI).

Distribution in China: S: Taiwan. Endemic.

Oreomyrrhis nanhuensis Chen Chihhsiung et Wang Jennche, 2001, Bot. Bull. Acad. Sin. (Taipei) 42(4): 308, fig. 5.

$\equiv$ Chaerophyllum nanhuense (Chen Chihhsiung et Wang Jennche) Chung Kuofang, 2007, Syst. Bot. 32(3): 679.

Type: CHINA. "Taiwan, Ilan Hsien, Tatung Hsiang, Taroko National Park, Nanhutashan cirque, ca 3400-3600 m, 23. 06. 1998, Chen Chihhsiung et al. 2403" (holotype TNU).

Distribution in China: S: Taiwan. Endemic.

Oreomyrrhis taiwaniana Masam., 1938, Trans. Nat. Hist. Soc. Formosa 28: 139.

$\equiv$ Chaerophyllum taiwanianum (Masam.) Chung Kuofang, 2007, Syst. Bot. 32(3): 679.

Type: CHINA. "[Taiwan] Mt. Nankotaizan [Nanhutashan], 17. 07. 1937, Suzuki" (holotype TAI).

Distribution in China: S: Taiwan. Endemic.

Osmorhiza aristata (Thunb.) Rydb., 1894, Bot. Surv. Nebraska 3: 37.

$\equiv$ Chaerophyllum aristatum Thunb. ex A.Murray, 1784, Syst. Veg. (ed. 14): 288; Thunb., 1784, Fl. Jap.: 119.

$\equiv$ Myrrhis aristata (Thunb.) Spreng., 1813, Neue Schr. Naturf. Ges. Halle 2, 1 (Pl. Umb. Prodr.): 29.

三Osmorhiza japonica Sieb. et Zucc., 1843, Abh. Akad. Muench. 4(2): 203, nom. illeg. (Art. 52.1) $\equiv$ Uraspermum aristatum (Thunb.) Kuntze, 1891, Revis. Gen. P1. 1: 270.

$\equiv$ Scandix aristata (Thunb.) Koso-Pol., 1916, Bull. Soc. Imp. Naturalistes Moscou, s.n. 29: 143.

Type: JAPAN. "[presumably from Japan, exact locality unknown]. Thunberg" (holotype UPSThunb.).

= Osmorhiza laxa Lindl., in Royle, 1835, Ill. Bot. Himal. Mts.: 233, tab. 52, fig. 1.

= Washingtonia laxa (Lindl.) Koso-Pol., 1920, Izv. Glavn. Bot. Sada RSFSR 36(1) (Fl. As. Ross. 15): 52.

= Osmorhiza aristata (Thunb.) Rydb. var. laxa (Lindl.) Shan Renhwa et Constance, 1948, Univ. Calif. Publ. Bot. 23(3): 130.

Type: INDIA. "Punjab, Simore in the Himalayan Mountains [NW India], Royle" (lectotype K! barcode K000695697, designated by Lowry, Jones, 1984: 1150; photo E; isolectotype K - barcode K000695688).

= Osmorhiza amurensis F. Schmidt ex Maxim., 1859, Mém. Acad. Imp. Sci. Saint-Petersbourg Divers Savants 9 (Prim. Fl. Amur.): 129.

$=$ Scandix amurensis (F. Schmidt ex Maxim.) Koso-Pol., 1916, Bull. Soc. Imp. Naturalistes Moscou, s.n. 29: 143.

= Washingtonia amurensis (F. Schmidt ex Maxim.) Koso-Pol., 1920, Izv. Glavn. Bot. Sada RSFSR 36, 1 (Fl. As. Ross. 15): 50, nom. inval., pro syn.

Type: RUSSIA. "Am untern Amur, in der Nähe der Dondon-Mündung, bei Dshare, an schattigen Laubwaldstellen, zerstreut, 18. 07. 1855, Maximowicz" (lectotype LE, designated here; isolectotype K! - barcode K000695689).

= Washingtonia claytonii (Michx.) Britton subsp. orientalis Koso-Pol., 1920, Izv. Glavn. Bot. Sada RSFSR 36, 1 (Fl. As. Ross. 15): 51.

Type: not indicated.

= Washingtonia claytonii (Michx.) Britton subsp. occidentalis Koso-Pol., 1920, Izv. Glavn. Bot. Sada RSFSR 36, 1 (Fl. As. Ross. 15): 51.

Type: not indicated.

- Osmorhiza longistylis auct. non DC.: Miq., 1867, Ann. Mus. Bot. Lugduno-Batavi 3: 64.

- Osmorhiza claytonii auct. non Michx.: C. B. Clarke, 1879, in Hooker f., Fl. Brit. India 2: 690.

Distribution in China: NW: Gansu; Tibet: Xizang A. R.; N: Ningxia Huizu A.R., Shaanxi, Shanxi, Hebei; NE: Heilongjiang, Jilin, Liaoning; Centr.: Henan, Jiangsu, Anhui, Hubei, Jiangxi, Zhejiang; SW: Sichuan, Guizhou, Yunnan; S: Taiwan.

Distribution outside China: Russia, Japan, Korea, Bhutan, Nepal, India, Pakistan, Kazakhstan. 
Note: Koso-Poljansky (1920) distinguished within Washingtonia claytonii, besides two subspecies cited above, also two varieties, differing in indumentum, and two forms, differing in involucre development. I doubt they could be treated as taxa.

Ostericum atropurpureum Li Genyou, Xia Guoxia et Xie Wenyuan, 2013, Nordic J. Bot. 31(4): 414.

Type: CHINA. "Zhejiang Province, Yuyuo County, Mt. Simingshan, growing in a mixed pineoak forest, $29^{\circ} 43^{\prime} 38.34^{\prime \prime} \mathrm{N}, 121^{\circ} 04^{\prime} 53.15^{\prime \prime} \mathrm{E}, 811$ m a.s.1., 11. 10. 2010, Xie Wenyuan, Li Genyou, Xia Guoxia 0128" (holotype ZJFC).

Distribution in China: Centr.: Zhejiang. Endemic.

Ostericum citriodorum (Hance) Yuan Changchi et Shan Renhwa, 1985, Bull. Nanjing Bot. Gard. Mem. Sun Yat Sen, 1984-85: 3.

$\equiv$ Angelica citriodora Hance, 1871, J. Bot. 9: 131.

Type: CHINA. "Ad cacumine montium Pakwan, supra Cantonem, 08. 10. 1869, Sampson, Hance 16393" (lectotype BM!, designated here; isolectotypes K! - barcode K001097164, LE!, W!).

Distribution in China: Centr.: Hubei, Hunan, Jiangxi, Zhejiang, Fujiang; S: Guangxi Zhuang A. R., Guangdong. Endemic.

Ostericum grosseserratum (Maxim.) Kitag., 1936, J. Jap. Bot. 12(4-5): 233.

$\equiv$ Angelica grosseserrata Maxim., 1873, Bull. Acad. Imp. Sci. Saint-Petersbourg 19: 275.

Type: CHINA. "In China boreali, non procul a Pekini, in montibus prope coemeterium militare Dun-lin, 08. 1856, Tatarinov" (holotype LE!).

= Angelica mongolica Franch., 1883, Nuov. Arch. Mus. Hist. Nat. (sér. 2) 6 (Pl. David. 1): 141.

Type: CHINA. "Mongolie: Gehol, bords des ruisseaux des montagnes, 09. 1864, David 2180" (lectotype P! - barcode P02573483, designated here; isoclectotypes $\mathrm{P}$ - barcodes P02573482, P02573484).

= Angelica koreana Maxim., 1886, Bull. Acad. Imp. Sci. Saint-Petersbourg 31: 51.

= Ostericum koreanum (Maxim.) Kitag., 1936, J. Jap. Bot. 12(4-5): 235.

Type: RUSSIA. "Ad Koreae limites: locis lapidosis circa sinum Possiet [Mandshuria austroorientalis, Bai Victoria, Possjet],12/24. 09. 1860, Maximowicz" (lectotype LE!, designated by Vinogradova, 2006: 1280; isolectotypes LE!).
= Angelica uchiyamae Yabe, 1903, Bot. Mag. (Tokyo) 17: 107.

Type: KOREA. "Phyöng-an-do: Muranbong, 12-20. 08. 1902, Uchiyama" (holotype TI!).

= Peucedanum mirabile $\mathrm{H}$. Wolff, 1925, Repert. Spec. Nov. Regni Veg. 21: 248.

Type: KOREA. "Corea: Quelpaert, in herbidis, 20. 10. 1908, Taquet 846" (holotype E).

= Angelica peucedanoides H. Wolff, 1926, Acta Horti Gothob. 2: 321.

Type: CHINA. "Chili: Hsiao-wu-tai-shan, Yangkia-p'ing, Pei-ko, auf feuchten Feldern, 11. 09. 1921, H. Smith 610" (lectotype UPS!, designated here; isolectotypes GB! - barcode GB0048797, W!).

= Ligusticum wawrae H. Wolff, 1930, Repert. Spec. Nov. Regni Veg. 27: 318.

Type: CHINA. "Peking, Tse-tai-ssu, Wawra 1065" (holotype W!).

Distribution in China: Tibet: Qinghai; N: Shaanxi, Shanxi, Hebei, Shandong; NE: Jilin, Liaoning; Centr.: Henan, Jiangsu, Anhui, Hubei, Jiangxi, Zhejiang, Fujiang; SW: Sichuan.

Distribution outside China: Russia, Korea.

Ostericum huadongense Pan Zehui et Li Xinhua, 1996, J. Pl. Resourc. Environ. 5(2): 45, "huadongensis".

Type: CHINA. "Anhui: Guangde, Baidian, alt. 260 m, roadside, 06. 10. 1992, Li Xinhua 92006" (holotype NAS).

Distribution in China: Centr.: Jiangsu, Anhui, Zhejiang. Endemic.

Ostericum longipedicellatum (H. Wolff) Pimenov et Kljuykov, 2003, Willdenowia 33(1): 128, fig. 4.

$\equiv$ Porphyroscias longipedicellata H. Wolff, 1930, Repert. Spec. Nov. Regni Veg. 27: 306.

$\equiv$ Angelica longipedicellata (H. Wolff) M. Hiroe, 1979, Umbelliferae World: 1430.

Type: CHINA. "Yunnan, on the Tong-Shan in the Yangtze bend. Lat. 27²0', 10000', 08. 1913, Forrest 10949" (lectotype E!, designated here; isolectotypes BM, K!).

Distribution in China: SW: Yunnan. Endemic.

Ostericum maximowiczii (F. Schmidt ex Maxim.) Kitag., 1936, J. Jap. Bot. 12(4-5): 232.

$\equiv$ Gomphopetalum maximowiczii F. Schmidt ex Maxim., 1859, Mém. Acad. Imp. Sci. SaintPetersbourg Divers Savans 9 (Prim. Fl. Amur.): 126.

$\equiv$ Angelica maximowiczii (F. Schmidt ex Maxim.) Benth. ex Maxim., 1873, Bull. Acad. Imp. Sci. Saint-Petersbourg 19: 274. 
Type: RUSSIA. "Mandshuria, ad fl. Amur [bei Tschjutscha unterhalb der Garin-Mündung, 04. 08. 1855, Maack" (lectotype LE!, designated by Vinogradova, 2006: 1282); "In der Küstenregion: im Nadelwalde um die Bai de Castries, 24. 07. 1854, Maximowicz" (syntype LE!); “Am untern Amur: in Gehölzen zwischen Nikolajevsk und Tschhirkrach, 22. 08. 1854, Maximowicz" (syntype LE!); "In hohen Amurufer gegenüber Nikolajevsk, 18. 08. 1854, Maximowicz" (syntype LE!); "Im Nadelwalde um Kitsi und Kotton (Mariinsk), 01. 09. 1854, Maximowicz" (syntype LE!); "Im Bureja-Gebirge, am Fusse nach Nord gelegener Abhänge unweit Chinganskoi Piket, 20. 08. 1856, Maximowicz" (syntype LE!) (Vinogradova, 2006: 1282; Yi U Ch'ol, 2008: 699).

= Angelica maximowiczii (F. Schmidt) Benth. ex Maxim. f. australis Kom., 1907, Trudy Imp. S.-Peterburgsk. Bot. Sada 25 (Fl. Mansh. 3): 165.

$=$ Ostericum maximowiczii (F. Schmidt ex Maxim.) Kitag. f. australe (Kom.) Kitag., 1936, J. Jap. Bot. 12 (4-5): 233.

= Ostericum maximowiczii (F. Schmidt) Kitag. var. australe (Kom.) Kitag., 1939, Rep. Inst. Sci. Res. Manch. 3(1) (Lin. fl. Manshur.): 340.

$=$ Angelica maximowiczii (F. Schmidt) Benth. ex Maxim. var. australis (Kom.) Gorovoj, 1966, Umbell. Prim. Priam.: 154, fig. 100.

Type: KOREA/CHINA. "In Korea septentr. et in Mansh. finitima".

Distribution in China: N: Neimenggu A. R.; NE: Heilongjiang, Jilin.

Distribution outside China: Russia, Korea.

Ostericum muliense (Shan Renhwa et Pu Fating) Pimenov et Kljuykov, 2003, Willdenowia 33(1): 129, fig. 5.

$\equiv$ Pachypleurum muliense Shan Renhwa et $\mathrm{Pu}$ Fating, 1989, Acta Phytotax. Sin. 27(1): 62, fig. 1.

Type: CHINA. "Sichuan, Muli, alt. 2600 m, in bush-woods by streams, 19. 08. 1978, Zhoo Qingheng 1569" (holotype CDBI!).

= Ostericum maximowiczii (F. Schmidt) Kitag. var. alpinum Shan Renhwa et Yuan Changchi, 1985, Bull. Nanjing Bot. Gard. Mem. Sun Yat Sen, 198485: 3.

Type: CHINA. "Sichuan: Hatoowan, Muli Xian, alt. 2250 m, 23. 10. 1982, Pu Fating, Yao Gan 386" (holotype NAS; isotype CDBI!).

Distribution in China: SW: Sichuan. Endemic.

Ostericum scaberulum (Franch.) Yuan Changchi et Shan Renhwa, 1985, Bull. Nanjing Bot. Gard. Mem. Sun Yat Sen, 1984-85: 3. $\equiv$ Angelica scaberula Franch., 1894, Bull. Annuel Soc. Philom. Paris (sér. 8) 6: 144.

Type: CHINA. "Yun-nan, in dumetis ad inram basin montis Ma-eul-chan, alt. $2300 \mathrm{~m}$. [Les broussailes au pied du Ma eul chan a $2300 \mathrm{~m}$ d'alt., 09. 09. 1889, Delavay 3901" (lectotype P! - barcode P02573468, designated here; isolectotypes K! barcode K001097149, P! - barcodes P02573469, P02573470, P02573474, P02573477); "Yun-nan, in paludosis ad Gnou-hay [ Les marais de Gnou Kay], 05. 08. 1889, Delavay 3937" (syntype P! - barcode P02573476).

Distribution in China: SW: Sichuan, Yunnan. Endemic.

Ostericum sieboldii (Miq.) Nakai, 1942, J. Jap. Bot. 18: 219.

三Peucedanum sieboldii Miq., 1867, Ann. Mus. Bot. Lugduno-Batavi 3: 63; 1867, Prolus. Fl. Jap.: 251.

Type: JAPAN. “Japonia, Siebold” (holotype L!). = Angelica miqueliana Maxim., 1873, Bull. Acad. Sci. Petersb. 19: 276.

= Peucedanum miquelianum (Maxim.) H. Wolff, 1925, Repert. Spec. Nov. Regni Veg. 21: 248.

= Ostericum miquelianum (Maxim.) Kitag., 1936, J. Jap. Bot. 12(4-5): 236.

Type: JAPAN. "In Kiusiu inferiore: silvis frondosis udis jugi Kundsho-san, 10. 1863, Maximovicz" (syntype LE!); "Nippon, jugo Hakone, 10. 1863, Maximovicz" (syntypes C! LE!, P! - barcode P02573487); "Godeng, 10. 1862, Maximowicz" (lectotype LE!, designated by Grubov in LE (Vinogradova, 2004: 27); isolectotype C!); "Nagasaki, 1863, Maximowicz" (syntype P! - barcodes P00835232, P00835233); "Kanazawa et Yokohama, in silvis, 09-10. 1862, Maximowicz" (syntypes C - barcode C10008501, LE!, P! - barcodes P02573488, P02573489); "Nippon media, Prov. Nambu, Tschonoski [Sugawa] 154" (syntypes C! barcodes C10008501, C10008502, FI!, LE!).

= Ligusticum nipponicum H. Wolff, 1925, Repert. Spec. Nov. Regni Veg. 21: 245.

Type: JAPAN. "Nippon, secus litis maris, Annori [Aomori, Honshu], 10. 1902, Faurie 5004" (holotype E?).

Distribution in China: N: Neimenggu A. R., Shaanxi, Shanxi, Hebei, Shandong; NE: Heilongjiang, Jilin, Liaoning; Centr.: Jiangsu, Anhui, Zhejiang.

Distribution outside China: Russia, Japan, Korea. 
Ostericum tenuifolium (Pall. ex Spreng.) Chu Youchang, 1995, Clavis P1. Chinae Bor.-Or. (ed. 2): 478, "tenuifolia".

$\equiv$ Athamanta tenuifolia Pall. ex Spreng., 1820, in Roemer \& Schultes (eds.), Syst. Veg. (ed. 15 bis) 6: 495.

$\equiv$ Libanotis tenuifolia (Pall. ex Spreng.) DC., 1830, Prodr. 4: 151.

$\equiv$ Angelica tenuifolia (Pall. ex Spreng.) Pimenov, 1985, Bot. Zhurn. (Leningrad) 70(11): 1494, fig. 1.

Type: RUSSIA. "In Sibiria versus Ochotsk. Herb. Pallas [Steller?]" (holotype B-Willd!; isotype HAL?).

= Peucedanum salinum Pall. ex Spreng. (ed.), 1824, Syst. Veg. (ed. 16) 1: 910.

Type: RUSSIA. "Sibir."

= Oreoselinum humile Besser ex Steud., 1840, Nomencl. bot. (ed. 2) 1: 226.

= Peucedanum humile Turcz. [1838, Bull. Soc. Imp. Naturalistes Moscou 11: 93, nom inval. (Art. 39.1)], 1844, Bull. Soc. Imp. Naturalistes Moscou 17: 745 .

Type: RUSSIA. "[Baikal et Transbaikalia] In umbrosis sylvaticis ad rivulorum margines [Ircutia], 1830, 1831, Turczaninow" (syntypes FI!, K! barcode K001097159, M!, P!).

= Ostericum filisectum Chu Youchang et Liou, 1977, Fl. Pl. Herb. Chin. Bor.-Or. 6: 294, 245, tab. 99.

= Ostericum maximowiczii (F. Schmidt) Kitag. var. filisectum (Chu Youchang) Yuan Changchi et Shan Renhwa, 1985, Bull. Nanjing Bot. Gard. Mem. Sun Yat Sen, 1984-85: 3.

Type: CHINA. "Nei Mongol: Ergun Banner, 16. 08. 1951, Wang Zhan 1970" (holotype IFP).

Distribution in China: N: Neimenggu A. R.; NE: Heilongjiang.

Distribution outside China: Russia, Mongolia, Kazakhstan.

Ostericum viridiflorum (Turcz.) Kitag., 1936, J. Jap. Bot. 12(4-5): 235.

$\equiv$ Gomphopetalum viridiflorum Turcz. [ex Besser, 1834, Flora 17(1, Beibl.): 14, nom. inval. (Art. 39.1)], 1841, Bull. Soc. Imp. Naturalistes Moscou 14: 540.

$\equiv$ Angelica viridiflora (Turcz.) Benth. ex Maxim., 1873, Bull. Acad. Imp. Sci. Saint-Petersbourg 19: 275.

Type: RUSSIA. "In pratis humidis Dahuriae inter Czindant, Nerczinsk et Nerczinskoi Zavod [Ad margines rivulorum inter Czindant, Nerczinsk et Nerczinskoi Zavod], 1831, Turczaninow" (lectotype LE!, designated by Vinogradova (2005:
952); isolectotypes G! - barcodes G00359722, G00359723, K! - barcode K001097158, LE!, M barcode M0173107, P! - barcodes P03239500, P03239501, P03239502); “ In pratis Dahuriae. 1831. Turczaninow" (syntype LE!); "Dahuria. 1832. Turczaninow" (syntype LE!).

Distribution in China: N: Neimenggu A. R.; NE: Heilongjiang, Jilin, Liaoning.

Distribution outside China: Russia.

Pachypleurum alpinum Ledeb., 1829, Fl. Altaic. 1: 297.

इLigusticum alpinum (Ledeb.) Kurtz, 1894, Bot. Jahrb. Syst. 19: 464, nom. illeg., non Spreng. (1824).

$\equiv$ Ligusticum mutellinoides (Crantz) Vill. subsp. alpinum (Ledeb.) Thell., 1926, in Hegi (ed), Ill. Fl. Mittel.-Eur. 5(2): 1323.

Type: KAZAKHSTAN. "In monte crucis prope Riddersk, regionis subalpinis, Ledebour 248"(syntypes LE!, P! - barcode P03776963, PH?). RUSSIA. "[Altai] In alpe Aigulacense. Bunge" (lectotype LE!, designated by Vinogradova, 2005: 963; isolectotypes $\mathrm{K}$ - barcode K000695883, P! barcodes P02284825, P03776972, P03776974).

= Conioselinum gayoides Less., 1834, Linnaea 9: 178.

Type: RUSSIA. "In madidis submuscosis montis Iremel per totam regionem alpinam, altit. 40004700 ped.,1832, Lessing" (holotype LE?).

= Pachypleurum schischkinii Serg., 1956, Sist. Zametki Mater. Gerb. Krylova Tomsk Gosud. Univ. 79-80: 7.

= Pachypleurum alpinum Ledeb. var. schischkinii (Serg.) Malyschev, 1965, Vysokogorn. Fl. Vostochn. Sajana: 188.

Type: KAZAKHSTAN. "Kasachstania orientalis. Systema flum. Kaba, Muzbel, in pratis alpinis, 14. 07. 1920, Saposhnikov" (lectotype TK!, designated by Polozhij, Balashova (1989: 26), as "type"; isolectotypes TK).

- Ligusticum mutellinoides auct. non Vill.: Hultén, 1929, Fl. Kamtchatka 3: 164, map 591.

Distribution in China: NW: Xinjiang Weiwuer A. R.

Distribution outside China: Russia, Mongolia, Kazakhstan. Europe.

Palimbia defoliata (Ledeb.) Korovin, 1963, in Pavlov (ed.), Fl. Kazakhst. 6: 382.

ESeseli defoliatum Ledeb., 1829, Fl. Altaic. 1: 343.

Type: KAZAKHSTAN. "In arenoso-limosis salsis deserti soongoro-kirghisici occidentalis [Inter fl. Irtysch et montes Tschingistau], 06. 1826, 
Meyer 267 (369.17)" (lectotype LE!, designated by Vinogradova, 2002b: 134; isolectotypes C! - barcode C10008579, P!, PH - barcode PH00023775).

Distribution in China: NW: Xinjiang Weiwuer A. R.

Distribution outside China: Kazakhstan.

Paraligusticum discolor (Ledeb.) V. N. Tikhom., 1973, Byull. Moskovsk. Obšč. Isp. Prir. Otd. Biol. 78(1): 107, fig. 4-7.

三Ligusticum discolor Ledeb., 1829, Fl. Altaic. 1: 321 .

$\equiv$ Pleurospermum discolor (Ledeb.) M. Hiroe, 1958, Umbell. Asia 1: 122.

Type: KAZAKHSTAN. "In pratis v.gr. prope Riddersk, Ledebour 233" (syntype LE!); "Alexandrowsk, 21. 06. 1826, Meyer 266" (lectotype LE!, designated by Vinogradova, 2005: 963; isolectotype $\mathrm{P}$ - barcode P03224819); "Pagum Belaya ad fl. Buchtorma, Ledebour" (syntype LE!).

Distribution in China: NW: Xinjiang Weiwuer A. R.

Distribution outside China: Mongolia, Kazakhstan, Kirghizia, Tajikistan, Uzbekistan.

Pastinaca sativa L. subsp. sylvestris (Mill.) Rouy et Camus, 1901, in Rouy \& Foucaud (ed.), F1. France 7: 372, "silvestris".

$\equiv$ Pastinaca sylvestris Mill., 1768, Gard. Dict. (ed. 8): Pastinaca N 1.

$\equiv$ Pastinaca sativa L. var. sylvestris (Mill.) DC., 1830, Prodr. 4: 189.

Type: EUROPE, GREAT BRITAIN. "On the side of banks, and on dry land, in many parts of England".

$=$ Pastinaca sativa L. var. arvensis Pers., 1805, Syn. Pl. 1: 322.

Distribution in China: Centr.: Jiangsu.

Distribution outside China: Russia, Japan, Kazakhstan, Kirghizia, Uzbekistan, Turkey. Europe, Africa, N. America, S. America, Australia and Oceania.

Pastinacopsis glacialis Golosk., Bot. Mater. Gerb. Bot. Inst. Komarova Akad. Nauk SSSR 12: 198. 1950.

三Pastinaca glacialis (Golosk.) M. Hiroe, 1979, Umbelliferae World: 1759.

Type: KAZAKHSTAN. "Alatau Transiliensis, angustiae fl. M. Alma-Atinka, morenae recentes glacierum Tujuk-su, declivum lapidosum, $3850 \mathrm{~m}$, 06. 08. 1943, Goloskokov" (holotype LE!; isotypes AA!, TASH!).
A. R.

Distribution in China: NW: Xinjiang Weiwuer

Distribution outside China: Kazakhstan, Kirghizia.

Peucedanum ampliatum Fu Kuntsun, 1981, Fl. Tsinling. 1(3): 462, 427, fig. 363.

Type: CHINA. "Shensi: Shan-yang Hsien, Tientu-shan, alt. $1660 \mathrm{~m}, 03.07 .1964$, Y. M. Liang 3126" (holotype WUK).

Distribution in China: N: Shaanxi. Endemic.

Peucedanum dielsianum Fedde ex H. Wolff, 1934, Repert. Spec. Nov. Regni Veg. 33: 246.

Type: CHINA. "Hupeh, Ichang, Henry 4381".

Distribution in China: Centr.: Hubei; SW: Sichuan. Endemic.

Peucedanum diversifolium H. Wolff, 1934, Repert. Spec. Nov. Regni Veg. 33: 247.

$\equiv$ Peucedanum chinense M. Hiroe, 1979, Umbelliferae World: 1572, nom. illeg., "chinensis".

Type: CHINA. "Western-China, Woodlands, Wilson 3671, 3705".

Distribution in China: SW. Endemic.

Peucedanum guangxiense Shan Renhwa et Sheh Menglan, 1986, Acta Phytotax. Sin. 24(4): 308, fig. 5, "quangxiense".

Type: CHINA. "Guangxi: Jingxi, alt. 300 m, 08. 11. 1961, X. F. Wu 23493" (holotype NAS!).

Distribution in China: S: Guangxi Zhuang A. R. Endemic.

Peucedanum harrysmithii Fedde ex H. Wolff, 1933, Repert. Spec. Nov. Regni Veg. 33: 247.

Type: CHINA. "Shansi centr.: Chieh-hsiuh Distr. Sung-lin-miao, Cho mei shan, 1000', in prato aprico, 03. 10. 1924, H. Smith 7609" (lectotype UPS!, designated here; isolectotypes MO - barcode MO-150814, PE!).

= Peucedanum praeruptorum Dunn subsp. hirsutiusculum Ma Yuchuan, 1979, Fl. Intramong. 4: 208, 198, tab. 92.

= Peucedanum hirsutiusculum (Ma Yuchuan) Shan Renhwa et Sheh Menglan [1986, Acta Phytotax. Sin. 24, 4: 310, comb. inval.] ex V. M. Vinogr., 1994, Rast. Tsentr. Azii 10: 64.

Type: CHINA. "Nei Monggol, Daqinshan, Halawugou, alt. $1300 \mathrm{~m}$, by valley stream, 16. 08 . 1964, Ma Yuchuan 64007" (holotype HIMC).

Distribution in China: NW: Gansu; N: Neimenggu A. R., Ningxia Huizu A. R., Shaanxi, 
Shanxi, Hebei; Centr.: Henan; SW: Sichuan. Endemic.

Peucedanum harrysmithii Fedde ex H. Wolff var. grande (Fu Kuntsun) Shan Renhwa et Sheh Menglan, 1992, in Fl. Reipubl. Popularis Sin. 55(3): 164.

$\equiv$ Peucedanum praeruptorum Dunn var. grande Fu Kuntsun, 1981, in Fl. Tsinling. 1(3): 428.

Type: CHINA. "Shensi: Hwa-in Hsien, Hwashan, alt. 1580 m, 05. 08. 1973, Fu Kuntsun 16884" (holotype WUK).

Distribution in China: N: Shaanxi. Endemic.

Peucedanum harrysmithii Fedde ex H. Wolff var. subglabrum (Shan Renhwa et Sheh Menglan) Shan Renhwa et Sheh Menglan, 1992, in Fl. Reipubl. Popularis Sin. 55(3): 164.

$\equiv$ Peucedanum hirsutiusculum (Ma Yuchuan) Shan Renhwa et Sheh Menglan var. subglabrum Shan Renhwa et Sheh Menglan, 1986, Acta Phytotax. Sin. 24(4): 310.

Type: CHINA. "Henan: Song Xian, alt. 1000 m, 15. 09. 1965, P. G. Wu, W. L. Wang 651161" (holotype NAS).

Distribution in China: N: Shanxi, Hebei; Centr.: Henan. Endemic.

Peucedanum henryi H. Wolff, 1933, Repert. Spec. Nov. Regni Veg. 33: 248.

Type: CHINA. "Hupeh: Ichang, 18851888, Henry 3604" (lectotype BM! - barcode BM000885451, designated here; isolectotypes P barcodes P02272030, P02272031, US! - barcode US00127464).

Distribution in China: Centr.: Hubei. Endemic.

Peucedanum japonicum Thunb., 1783, Nova Acta Regiae Soc. Sci. Upsal. 4: 38; ex A. Murray, 1784, Syst. Veg. (ed. 14): 280; Thunb., 1784, Fl. Jap.: 117.

$\equiv$ Anethum japonicum (Thunb.) Koso-Pol., 1916, Bull. Soc. Imp. Naturalistes Moscou, s.n. 29: 117.

Type: JAPAN. "Crescit in littoribus maris circa Nagasaki et adiacentium regionum insularumque, Kosido, Papenberg et al." (UPS?).

$=$ Peucedanum japonicum Thunb. var. purpurascens Makino, 1926, J. Jap. Bot. 3(6): 24.

=Peucedanumjaponicum Thunb. f.purpurascens (Makino) Honda, 1939, Nom. Pl. Japon.: 250, 512.

Type: JAPAN. "Hayama, prov. Sagami, 1926, Makino".

Distribution in China: N: Shandong; Centr.: Jiangsu, Zhejiang, Fujiang; S: Taiwan.
Distribution outside China: Japan, Korea, Philippines.

Peucedanum japonicum Thunb. f. album Q. H. Yang et Q. Tian, 2008, Acta Bot. Boreal.-Occid. Sin. 28(2): 399.

Type: CHINA. "Zhejiang, Dongbailian Island, 8 m. 22. 06. 2007, Q. H. Yang, Q. Tian 07-0331" (holotype CSH).

Distribution in China: Centr.: Zhejiang. Endemic.

Peucedanum ledebourielloides $\mathrm{Fu}$ Kuntsun, 1981, Fl. Tsinling. 1(3): 463, 428, fig. 365.

Type: CHINA. "Shensi: Hwa Shan, Wu-likwan, alt. 650 m, 19. 09. 1973, Wang 22" (holotype WUK).

Distribution in China: N: Shaanxi; Centr.: Henan. Endemic.

Peucedanum longshengense Shan Renhwa et Sheh Menglan, 1986, Acta Phytotax. Sin. 24(4): 306, fig. 3.

Type: CHINA. "Guangsi, Longshang, alt. 1850 m, 30. 09. 1956, S. L. Yu, J. F. Qing 700634" (holotype SCBI).

Distribution in China: Centr.: Hunan, Jiangxi; S: Guangxi Zhuang A. R. Endemic.

Peucedanum mashanense Shan Renhwa et Sheh Menglan, 1986, Acta Phytotax. Sin. 24(4): 304, fig. 1.

Type: CHINA. "Guangxi, Mashan, alt. $300 \mathrm{~m}$, 07. 09. 1958, S. Q. Zhong 301547" (holotype SCBI; isotype KUN!).

Distribution in China: S: Guangxi Zhuang A. R. Endemic.

Peucedanum medicum Dunn, 1903, J. Linn. Soc. Bot. 35: 496.

Type: CHINA. "Hupeh: Ichang and intermediate neighbourhood, 02. 1887, Henry 1546, 2006" (lectotype K! - barcodes K000685413, K000685414, designated here; isolectotypes BM!, E!, K - barcode K000685416, NY - barcode NY00406177, P! - barcode P00752971); "Hupeh, Nam-T'o, 1887, Henry 1906" (syntype K! - barcode K000685415); "Prov. Hupeh: Fang, Henry 5868A" (syntypes K! - barcode K000685417, LE!, NY barcode NY00406176, P! - barcode P00752968); "Szechuen: South Wushan, Henry 7473" (syntypes LE!, P! - barcode P02272028). 
Distribution in China: Centr.: Hubei, Hunan, Jiangxi; SW: Sichuan, Guizhou; S: Guangxi Zhuang A. R., Guangdong. Endemic.

Peucedanum medicum Dunn var. gracile Dunn ex Shan Renhwa et al., 1986, Acta Phytotax. Sin. 24(4): 310 .

Type: CHINA. "Szechuan, Wulong, alt. 1100 m, 27. 09. 1964, Sheh Menglan, Yuan Changchi, S. L. Huang 6468" (holotype NAS!).

Distribution in China: SW: Sichuan. Endemic.

Peucedanum morisonii Besser ex Schult., in Roemer \& Schultes (eds.), 1820, Syst. Veg. (ed. 15 bis) $6: 567$.

= Ligusticum longifolium Willd., 1798, Sp. pl. (ed. 4), 1 (2): 1428, non Peucedanum longifolium Waldst. et Kit.

= Peucedanum ledebourii G. Don, 1834, Gen. Hist. 3: 330.

Type: RUSSIA. "Native in Siberia, everywhere in open situations, particularly on rocks" (holotype B-Willd).

= Peucedanum songoricum Schischk., 1951, in Schischkin (ed.), Fl. URSS 17: 354, 176, nom. illeg., non Peucedanum soongaricum G. Don (1834).

Type: KAZAKHSTAN. "Kazachstania. In jugo Dshungarski Alatau, montes Bajan-Dshurjuk, 03. 07. 1909, Lipsky 1798" (holotype LE!).

- Peucedanum officinale auct. non L.: Ledeb., 1829, Fl. Altaic. 1: 304.

- Peucedanum ruthenicum auct. non M. Bieb.: Krylov, 1935, Fl. Zapadnoi Sibiri 8: 2045.

Distribution in China: NW: Xinjiang Weiwuer A. R.

Distribution outside China: Russia, Kazakhstan.

Peucedanum muliense Sheh Menglan, 1992, J. P1. Resourc. Environ. 1(3): 3.

Type: CHINA. "Sichuan, Muli, alt. 4000 m, 22. 10. 1937, T. T. Yu 14605" (holotype PE).

Distribution in China: China (SW: Sichuan). Endemic.

Peucedanum nanum Shan Renhwa et Sheh Menglan, 1980, Acta Phytotax. Sin. 18(3): 377.

Type: CHINA. "Xizang, Lhasa, in clivis montibus, alt. 3500-3700 m, 16. 09. 1970, Kuo 8109" (holotype HNWP; isotype NAS!).

Distribution in China: Tibet: Xizang A. R. Endemic.
Peucedanum pubescens Hand.-Mazz., 1933, Symb. Sin. 7(3): 728, tab. 12, fig. 5.

Type: CHINA. "Prov. Yunnan centralis: In regionis calide temperatae ad orientem fluminis Dsolin-ho, declivibus siccis inter vicos Mabou schan et Bölu, 1900-2000 m, 09. 11. 1916, HandelMazzetti 13043" (lectotype WU! - barcode WU0029560, designated here; isolectotypes E barcode E00002620, W!).

Distribution in China: SW: Sichuan, Yunnan. Endemic.

Peucedanum rubricaule Shan Renhwa et Sheh Menglan, 1986, Acta Phytotax. Sin. 24(4): 305, fig. 2, nom. illeg. (Art. 53.1), non Baill. (1879).

Type: CHINA. "Sichuan: Huidong, alt. 2000 3000 m, 09. 10. 1964, Yue Jinsan, K. J. Song, T. D. Zhuang 64199" (holotype NAS!).

Distribution in China: SW: Sichuan, Yunnan. Endemic.

Peucedanum songpanense Shan Renhwa et $\mathrm{Pu}$ Fating, 1989, Acta Phytotax. Sin. 27(1): 65, fig. 4.

Type: CHINA. "Sichuan: Songpan, alt. 2800$3000 \mathrm{~m}$, under thining forests or on side of agrarian field, 06. 10. 1983, Pu Fating 056" (holotype CDBI!).

Distribution in China: SW: Sichuan. Endemic.

Peucedanum violaceum Shan Renhwa et Sheh Menglan, 1980, Acta Phytotax. Sin. 18(3): 378.

Type: CHINA. "Xizang, Mainling Xian, in locis arenosis montis Do Hsium, alt. 2980 m, 11. 08. 1975, Qinghai-Xizang Exped. 751309" (holotype $\mathrm{PE}$; isotype KUN!).

Distribution in China: Tibet: Xizang A. R. Endemic.

Peucedanum wulongense Shan Renhwa et Sheh Menglan, 1986, Acta Phytotax. Sin. 24(4): 309, fig. 6.

Type: CHINA. "Wulong Baima, Sichuan, alt. 580 m, 26. 09. 1964, Sheh Menglan, Yuan Changchi, S. L. Huang 6455" (holotype NAS!).

Distribution in China: SW: Sichuan. Endemic.

Peucedanum yunnanense H. Wolff, 1925, Repert. Spec. Nov. Regni Veg. 21: 247.

Type: CHINA. "Prov. Yunnan, Yunnanfu, Cavalerie" (holotype P?).

Distribution in China: SW: Yunnan. Endemic.

Phlojodicarpus sibiricus (Fisch. ex Spreng.) Koso-Pol., 1922, Schedae Herb. Fl. Ross. 8: 117. 
$\equiv$ Cachrys sibirica Fisch. ex Spreng., 1824, Syst. Veg. (ed. 16) 1: 892.

$\equiv$ Angelica sibirica (Fisch. ex Spreng.) M. Hiroe, 1958, Umbell. Asia 1: 168.

Type: RUSSIA. "[Sibir] [ex Hort. Bot. Wratislav.], Herb. Mertens" (lectotype LE!, designated by Vinogradova, 2005: 960; isolectotype $\mathrm{P}$ !).

= Seseli vaginatum Ledeb., 1829, Fl. Altaic. 1: 336.

Type: RUSSIA. "[Transbaikalia] In Davuria prope Nertschinsk, Herb. Ledeb. 369.15" (holotype LE!).

=Libanotis cachroides DC., Prodr. 4: 151. 1830.

$=$ Peucedanum cachroides (DC.) Koso-Pol., 1920, Izv. Glavn. Bot. Sada RSFSR 36(1) (Fl. As. Ross.15): 24.

Type: RUSSIA. "In Dahuria prope Nerstinski Savod" (holotype G-DC!).

= Phlojodicarpus davuricus Turcz. ex Ledeb., 1844, Fl. Ross. 2: 331.

= Phlojodicarpus eudahuricus Popov, 1957, Fl. Srednei Sibiri 1: 174.

Type: RUSSIA. "In lapidosis Sibiriae transbaicalensis [Nertschinsk], Turczaninow" (holotype KW!; isotypes M - barcode M0173083, $\mathrm{P}$ - barcode P03223941).

= Phlojodicarpus baicalensis Popov, 1955, Spisok Rast. Gerb. Fl. SSSR 13: 32.

= Phlojodicarpus popovii Sipliv., 1971, Novosti Sist. Vyssh. Rast. 7: 259.

Type: RUSSIA. "Lac. Baical, ripa occidentalis, prope pag. B. Koty, $52^{\circ}$ alt. bor., in declivibus lapidosis ad lacum, 07-08. 1952, Popova 3843" (holotype LE!; isotypes A - barcode A00077442, BM!, C! - barcode C10008511, DAO - barcode DAO00455722, E! - barcode E00002473, GH, JE barcodes JE00007099, JE00007100, K!, M! barcode M0173084, MHA!, MO - barcode MO345441, MW!, NSK - barcode NSK0000252, NY barcode NY00406182, S! - barcode S-G-4787, TK).

$=$ Phlojodicarpus sibiricus (Fisch. ex Spreng.)

Koso-Pol. var. villosus Chu Weiming, 1977, Pl. Herb. Chin. Bor.-Or. 6: 287, tab. 119, fig. 5-6, non Ph. villosus Turcz.

$=$ Phlojodicarpus villosus auct. non Ledeb.: Sheh Menglan, in Shan Renhwa et Sheh Menglan, 1992, Fl. Reipubl. Popularis Sin. 55(3): 121, tab. 51, fig. $1-6$.

=Phlojodicarpus baicalensis Popov var. arenarius Peschkova, 1972, Stepnaya Fl. Baikal'skoi Sibiri: 83, nom. inval. (Art. 39.1).

Distribution in China: N: Neimenggu A. R., Hebei; NE: Heilongjiang, Mongolia.
Distribution outside China: Russia, Mongolia.

Physospermopsis alepidioides (H. Wolff et Hand.-Mazz.) Shan Renhwa, 1936, Sinensia 7: 187, "alepioides".

$\equiv$ Haploseseli alepidioides $\mathrm{H}$. Wolff et Hand.Mazz., 1933, Symb. Sin. 7(3): 722, tab. 12, fig. 2-4.

Type: CHINA. "Prov. Setschwan austro-occid.: Zwischen Yenyuen und Kwapi, in Gebüschen der tr. St. zwischen Tangetu und Hwangliangdse, 27 ${ }^{\circ} 45^{\circ}$ N, 3300 m, 04. 10. 1914, Handel-Mazzetti 5483" (syntypes BM!, PE!, WU); "Prov. Setschwan austrooccid.: prope vicum Kalapa, 27 $40^{`}$ N, 2700-2800 $\mathrm{m}$, inter oppidum Venyiien et castellum Kwapi, in regionis temperatae et calide semp. limite steppis, substr. calceo, 07. 10. 1914, Handel-Mazzetti 5562" (syntypes WU!).

Distribution in China: SW: Sichuan. Endemic.

Physospermopsis delavayi (Franch.) H. Wolff, 1925, Notizbl. Bot. Gart. Berlin-Dahlem 9: 278.

= Arracacia delavayi Franch., 1894, Bull. Annuel Soc. Philom. Paris (sér. 8) 6: 115.

$=$ Pleurospermum delavayi (Franch.) M. Hiroe, 1958, Umbell. Asia 1: 120.

Type: CHINA. "Yun-nan, in collibus ad pedem montis Yang-in-chan, prope Mo-so-yn [Sur côteaux au pied du Yang in chan, près de Mo so pin (Lan Kong), 15. 09. 1885, Delavay 2017" (lectotype P! - barcode P00245424, designated by Pimenov, Kljuykov, 2000: 537); “ In pratis montis Pee-ngaytze [Prairies du Mt. Pee ngay tze (au dessus de Tapin-tze), 01. 09. 1882, Delavay 447" (syntype P! barcode P00245423).

Distribution in China: Centr.: Hubei; SW: Sichuan, Yunnan. Endemic.

Physospermopsis fuscopurpurea (Hand.-Mazz.) Pimenov et Kljuykov, 2000, Feddes Repert. 111(78): 538.

$\equiv$ Trachydium fuscopurpureum Hand.-Mazz., 1933, Symb. Sin. 7(3): 711.

Type: CHINA. "Prov. Yunnan bor--occid.: prope fines Tibeto-Birmanicas inter fluvios $\mathrm{Lu}-$ djiang (Salween) et Djiou-djiang (Irawadi or. sup.), in regione alpina retro montem Gomba-la supra Tschamutong, ad occid. lacus Tsukul. 4000-4100 m, 15-17. 08. 1916, Handel-Mazzetti 9900" ((holotype WU! (barcode WU0025957).

Distribution in China: SW: Yunnan. Endemic.

Physospermopsis handelii (H. Wolff ex Hand.Mazz.) Pimenov et Kljuykov, 2000, Feddes Repert. 111(7-8): 538. 
= Pleurospermum handelii H. Wolff, 1933, in Handel-Mazzetti, Symb. Sin. 7(3): 710, tab. 12, fig. 1.

Type: CHINA. "Prov. Yunnan bor--occid.: Prope fines Tibeto-Birmanicas inter fluvios Ludjiang (Salween) et Djion-djiang (Irawadi or. sup.), in regione alpina retro montem Gomba-la supra Tschamutong, ad occid. lacus Tsukue, 4000-4100 m, 15-17. 08. 1916, Collectores indigeni 9903" (lectotype WU! - barcode WU0025945, designated here; isolectotype E!).

Distribution in China: SW: Yunnan. Endemic.

Physospermopsis hirsutula (C. B. Clarke) Farille, 1985, Candollea 40(2): 513, fig. 1 (7-11).

$\equiv$ Trachydium hirsutulum C. B. Clarke, 1879, in Hooker f. (ed.), Fl. Brit. India 2: 672.

三Aulacospermum hirsutulum (C. B. Clarke) A. R. Naqshi, U. Dhar et Kachroo, 1995, Central Asia \& Kashmir Himalaya, Archeobot. \& Flor.: 107.

Type: INDIA. "Sikkim, Tungu, alt. 1300014000 ft., 10. 1849, Hooker" (lectotype K! barcode K000697265, designated here; isolectotype K! - barcode K000697266).

$=$ Physospermopsis farillei P. K. Mukh. et Constance, 1991, Edinburgh J. Bot. 48(1): 41.

Type: NEPAL. "C. Nepal, Lamjing Himal, au pied sud du col de Namun, vers 4000-4150 m, 26. 09. 1981, Farille 81-604" (holotype E!).

Distribution in China: Tibet: Xizang A. R.

Distribution outside China: Nepal, India.

Physospermopsis muliensis Shan Renhwa et Liou Shoulu, 1979, in Shan Renhwa \& Sheh Menglan (eds.), Fl. Reipubl. Popularis Sin. 55(1): 297, 105, tab. 48, fig. 1-5.

Type: CHINA. "Szechuan, Muli Hsien, 20. 10. 1937, T. T. Yu 14579” (holotype PE; isotype UC!).

Distribution in China: SW: Sichuan, Yunnan. Endemic.

Physospermopsis nana (Franch.) Pimenov et Kljuykov, 2000, Feddes Repert. 111(7-8): 538.

= Pleurospermum nanum Franch., 1894, Bull. Annuel Soc. Philom. Paris (sér. 8) 6: 110.

Type: CHINA. "Province du Yun-nan, in pratis humidis montis Tsong-chan, alt. $4000 \mathrm{~m}$ [Prairies humid. au bord du lac sur la Tsong-chan], 30. 08. 1889, Delavay 4066" (lectotype P! - barcode P00834546, designated by Pimenov, Kljuykov, 2000: 538; isolectotype P! - barcode P00834544, P00834547); "Sommet du Tsong-chan, au dessus de Ta-li, 25. 09. 1884, Delavay 197" (syntypes P! barcodes P00834544, P00834545).
Distribution in China: Tibet: Xizang A. R.; SW: Yunnan. Endemic.

Physospermopsis obtusiuscula (DC.) C. Norman, 1938, J. Bot. 76: 231.

三 Hymenolaena obtusiuscula DC., 1830, Prodr. 4: 246 .

ETrachydium obtusiusculum (DC.) C. B. Clarke, 1879, in Hooker f. (ed.), Fl. Brit. India 2: 673.

$\equiv$ Pleurospermum obtusiusculum (DC.) M. Hiroe, 1979, Umbelliferae World: 741.

三 Aulacospermum obtusiusculum (DC.) A. R. Naqshi, U. Dhar et Kachroo, 1995, Central Asia \& Kashmir Himalaya, Archeobot. \& Flor.: 107, nom. inval. (Art. 41.5), sine basyon.

Type: NEPAL. "Ad Gossain-Than Nepalensium, Wallich [543]" (lectotype G-DC!, designated by M. F. Watson, 1998: 376; isolectotypes BM - barcode BM000622303, BM000944782, CAL, K! - barcode K000697363, K-WALLICH!, LE!).

= Trachydium kingdon-wardii H. Wolff, 1929, Repert. Spec. Nov. Regni Veg. 27: 124.

$=$ Physospermopsis kingdon-wardii (H. Wolff)

C. Norman, 1938, J. Bot. 76: 231.

= Pleurospermum kingdon-wardii $(\mathrm{H}$. Wolff $) \mathrm{M}$. Hiroe, 1979, Umbelliferae World: 747.

Type: CHINA. "Tibet: A-tun-tsi, screes, turf, 14000 ft. s.m., 07. 08. 1913, Kingdon-Ward 992” (lectotype E, designated by M. F. Watson, 1998: 375).

Distribution in China: Tibet: Xizang A. R.; SW: Sichuan, Yunnan.

Distribution outside China: Bhutan, Nepal, India.

Physospermopsis purpurascens (Franch.) Pimenov et Kljuykov, 2000, Feddes Repert. 111(78): 538 .

三Trachydium purpurascens Franch., 1894, Bull. Annuel Soc. Philom. Paris (sér. 8) 6: 112.

Type: CHINA. "Yun-nan, ad collum montium Yen-tze-hay (Lan kong), alt. 3200 m [Sur côteaux, au col de Yen tze hay], 18. 09. 1885, Delavay 2015" (lectotype P! - barcode P00834548, designated as "type" in P by M. F. Watson; isolectotypes K!, P barcodes P00834549, P00834550).

Distribution in China: SW: Sichuan, Yunnan. Endemic.

Physospermopsis rubrinervis (Franch.) C. Norman, 1938, J. Bot. 76: 231.

三 Trachydium rubrinerve Franch., 1894, Bull. Annuel Soc. Philom. Paris (sér. 8) 6: 112. 
$\equiv$ Pleurospermum rubrinerve (Franch.) M. Hiroe, 1979. Umbelliferae World: 747.

Type: CHINA. "Yun-nan, in pascuis ad collum montis Lopin-chan, alt. $3200 \mathrm{~m}$ [Les paturages au col de Lo Pin chan], 31. 08. 1888, Delavay 3235" (lectotype P! - barcode P00245453, designated here; isolectotypes $\mathrm{K}$ !, $\mathrm{P}$ ! - barcodes P00245454, P00834665).

Distribution in China: SW: Sichuan, Yunnan.

Distribution outside China: Nepal.

Physospermopsis shaniana Wu Zhengyi et $\mathrm{Pu}$ Fating, 1993, in Wang Wentsai (ed.), Vasc. Pl. Hengduan Mount.: 1285.

$\equiv$ Trachydium forrestii Diels, 1912, Notes Roy. Bot. Gard. Edinburgh 5: 291.

$\equiv$ Physospermopsis forrestii (Diels) C. Norman, 1938, J. Bot. 76: 231, nom. illeg. (Art. 53.1), non Fedde ex H. Wolff (1929).

$\equiv$ Pleurospermum forrestii (Diels) M. Hiroe, 1958, Umbell. Asia 1: 123.

Type: CHINA. "Yunnan, shady, grassy openings in pine forests on the eastern flank of the Lichiang range. Lat. $27^{\circ} 20^{\prime}$ N. Alt.10-11000 ft., 08. 1906, Forrest 2855" (lectotype E! - barcode E00000219, designated here; isolectotype P! - barcode P00245432).

Distribution in China: Tibet: Xizang A. R.; SW: Sichuan, Yunnan.

Distribution outside China: Myanmar.

Pimpinella acuminata (Edgew.) C. B. Clarke, 1879, in Hooker f. (ed.), Fl. Brit. India 2: 686.

$\equiv$ Reutera acuminata Edgew., 1846, Trans. Linn. Soc. London 20(1): 52.

$\equiv$ Carum acuminatum (Edgew.) Franch., 1894, Bull. Annuel Soc. Philom. Paris (sér. 8) 6: 23, in obs.

Type: INDIA. "Himala, ad alt. ped. 5000-8000; near Chepal, 1844, Edgeworth 149" (lectotype K! barcode K000685502, designated here); "Himala, Masuri, Edgeworth 149" (syntype K! - barcode K000685501).

= Pimpinella hazariensis $\mathrm{H}$. Wolff, 1930, Repert. Spec. Nov. Regni Veg. 27: 331.

Type: PAKISTAN. "Kashmir: Kund, Hazara, 15. 08. 1899, Inayat Khan" (holotype DD!).

Distribution in China: Tibet: Xizang A. R., Qinghai; SW: Sichuan, Yunnan.

Distribution outside China: Nepal, India, Pakistan.

Pimpinella anisum L., 1753, Sp. pl.: 264.

$\equiv$ Apium anisum (L.) Crantz, 1767, Class. Umbell. Emend.: 101. $\equiv$ Anisum vulgare Gaertn., 1788, Fr. Sem. P1. 1: 102, tab. 23, fig. 1.

$\equiv$ Anisum officinarum Moench, 1794, Methodus: 100, nom. illeg. (Art.. 52.1)

三Sison anisum (L.) Spreng., 1812, Mag. Neuersten Entdesk. Gesammten Naturk. Ges. Naturf. Freude Berlin 6: 260.

$\equiv$ Tragium anisum (L.) Link, 1821, Enum. Hort. Berol. Alt. 1: 285.

$\equiv$ Carum anisum (L.) Baill., 1879, Hist. Pl. 7: 119, 178.

三Selinum anisum (L.) E. H. L. Krause, 1904, in Sturm (ed.), Fl. Deutschl. (ed. 2) 12: 56.

Type:AFRICA. "InAegypto, 373.10"(lectotypeLINN, designated by Yurtzeva, 1995: 66).

Distribution in China: cultivated in different regions.

Distribution outside China: Russia, Azerbaijan, Georgia, Armenia, Turkey, Oman, Yemen, Israel, Cyprus, Greece, Europe, Australia and Oceania (cult.).

Pimpinella asianensis M. Hiroe, 1979, Umbelliferae World: 833.

$\equiv$ Pimpinella engleriana Fedde ex H. Wolff, 1930, Repert. Spec. Nov. Regni Veg. 27: 320, nom. illeg. (Art. 52.1), non H. Wolff (1927).

$\equiv$ Pimpinella feddei Wu Wencheng et $\mathrm{Wu}$ Zhengyi, 1984, Index Fl. Yunnan. 1: 920, nom. illeg.

Type: CHINA. "Yunnan: Hills to the northwest of Teng Yueh, $25^{\circ} \mathrm{N}$ lat., on the margins of thickets, Forrest 8800" (holotype E! - barcode E00000447).

Distribution in China: SW: Yunnan. Endemic.

Pimpinella atropurpurea Wu Zhengyi, 1983, Acta Phytotax. Sin. 21(1): 81, tab. 1, fig. 1-5.

Type: CHINA. "Yunnan, Chengkang, Snow Range, alt. $3500 \mathrm{~m}$, on open and grassy slope, 02. 08. 1938, T. T. Yu 17136" (holotype PE!).

Distribution in China: SW: Yunnan. Endemic.

Pimpinella bialata H. Wolff, 1929, Repert. Spec. Nov. Regni Veg. 27: 188.

Type: CHINA. "Hubei, 09. 1907, Henry 2649".

Distribution in China: Centr.: Hubei. Endemic.

Pimpinella bisinuata H. Wolff, 1930, Repert. Spec. Nov. Regni Veg. 27: 332.

Type: CHINA. "Yunnan, Do ay Regions de Tsing Tchouan, 30. 08. 1911, Ducloux 7156".

Distribution in China: SW: Sichuan, Yunnan. Endemic.

Pimpinella candolleana Wight et Arn., 1834, Prodr. Fl. Penins. Ind.Orient. 1: 369. 
Type: INDIA. "Peninsular India. Mountains in the south of the Peninsula, 09. 1836, Wight 1173, 1194, 1202" (lectotype K!, designated here; isolectotypes E! - barcode E00174681, NY barcode NY00406195, P - barcode P00834778).

Distribution in China: SW: Sichuan, Guizhou, Yunnan; S: Guangxi Zhuang A.R., Guangdong.

Distribution outside China: Vietnam, India.

Pimpinella candolleana Wight et Arn. var. rotundifolia Shan Renhwa et Pan Zehui, 1997, in Wu Zhengyi (ed.), Fl. Yunnan. 7: 495, tab. 148, fig. 4-5.

Type: CHINA. "Yunnan, Yongren, 03. 08. 1976, Wu Zhengyi 4024" (holotype KUN).

Distribution in China: SW: Yunnan. Endemic.

Pimpinella caudata (Franch.) H. Wolff, 1927, in Engler (ed.), Pflanzenreich 90(IV, 228): 279.

$\equiv$ Carum caudatum Franch., 1894, Bull. Annuel Soc. Philom. Paris (sér. 8) 6: 126.

Type: CHINA. "Yun-nan, Ma-eul-chan, in silvis [Les bois de Ma eul chan], 10 .09. 1889, Delavay 3905" (lectotype P! - barcode P00752670, designated here; isolectotypes $\mathrm{K}$ ! - barcode K000685541, NY, P! - barcode P00752671).

Distribution in China: Tibet: Xizang A. R.; SW: Sichuan, Yunnan. Endemic.

Pimpinella chungdienensis Wu Zhengyi, 1980, Acta Phytotax. Sin. 18(3): 375.

Type: CHINA. "Yunnan: Zhongdian Xian, HaBa jugum, alt. $3100 \mathrm{~m}, 25.08$. 1962, Wu Zhengyi 1351" (holotype KUN; isotype PE!).

Distribution in China: Tibet: Xizang A. R.; SW: Sichuan, Yunnan. Endemic.

Pimpinella cnidioides H. Pearson ex H. Wolff, 1929, Repert. Spec. Nov. Regni Veg. 27: 183.

Type: CHINA. "Environs of Peking. Hort. Kew, 07. 1900, seeds from Vilmorin 1444" (lectotype K! barcode K000685526; isolectotypes $\mathrm{K}$ - barcodes K000685527, K000685528).

$=$ Pimpinella thellungiana $\mathrm{H}$. Wolff var. tenuisecta Chu Youchang, 1977, Fl. Pl. Herb. Chin. Bor.-Or. 6: 293, 208, tab. 82, fig. 6.

Type: CHINA. "Nei Mongghol, Horqin Youyi Qianqi, 11. 07. 1963, Wang Zhan 1439" (holotype IFP).

Distribution in China: N: Neimenggu A. R., Hebei; NE: Heilongjiang, Jilin. Endemic.

Pimpinella coriacea (Franch.) H. Boissieu, 1909, Bull. Soc. Bot. France 56: 351.
ECarum coriaceum Franch., 1894, Bull. Annuel Soc. Philom. Paris (sér. 8) 6: 127.

Type: CHINA. "Yun-nan, in pratis ad Hia-mati, supra Tapin-tze [Les prairies de Hia ma ti audessus de Ta pin tze], 04. 08. 1887, Delavay 2771" (lectotype P! - barcode P00854781, designated here; isolectotypes NY - barcode NY00405776, P! - barcodes P00854779, P00854780, P00854783 P00854784); "[Yunnan] Les prairies de Hee chan men au-dessus de Lan Kong], 05. 11. 1887, Delavay 445" (syntype P! - barcode P00834782).

= Pimpinella pseudocandolleana $\mathrm{H}$. Wolff, 1929, Repert. Spec. Nov. Regni Veg. 27: 189.

Type: CHINA. "Yunnan, Mengtze, 5000', in grass, Henry 9588" (lectotype K! - barcode K000685525, designated here; isolectotypes E! barcode E00000486, LE!,US).

= Pimpinella rockii $\mathrm{H}$. Wolff, 1929, Repert. Spec. Nov. Regni Veg. 27: 191.

Type: CHINA. "Yunnan: Yangtze watershad, prefectural district of Likiang, eastern slopes of Likiang Snow Range, alt. 13-14000 ft., 04-10. 1922, Rock 4533, 4635, 4669, 5225" (lectotype K! - barcode K000685523, designated here; isolectotypes $\mathrm{E}$ ! - barcode E00000503, K!, NY barcode NY00406188, US! - barcode US01107964).

= Pimpinella wolffiana Fedde ex H. Wolff, 1929, Repert. Spec. Nov. Regni Veg. 27: 185.

Type: CHINA. "Yunnan: Szemao, W mts. 5000' s.m., Henry 12485" (lectotype E! - barcode E00000494, designated here; isolectotypes K!, LE!).

Distribution in China: SW: Sichuan, Guizhou, Yunnan; S: Guangxi Zhuang A. R. Endemic.

Pimpinella crispulifolia H. Boissieu, 1909, Bull. Soc. Bot. France 56: 351.

Type: CHINA. "Yun-nan, Lao-Kouy-Chan, près Tsang-Le, 15. 07. 1906, Ducloux 4083" (holotype P! - barcode P00752662).

Distribution in China: SW: Yunnan. Endemic.

Pimpinella decursiva H. Wolff, 1920, Repert. Spec. Nov. Regni Veg. 16: 237.

Type: CHINA. "Tsingtau: Kaiserl. Oberforsterei Iltisberg, 232" (syntype); "Prov. Schantung, Clarabucht, am sandigen Meeresstrande, Krug 463".

Distribution in China: N: Shandong. Endemic.

Pimpinella diversifolia DC., 1830, Prodr. 4: 122. Type: INDIA. "[In Sirmore Indiae orientalis] introduced in Calcutta Bot. Garden, Wallich [574.2]" (lectotype G-DC! - barcode G00316025, designated by Watson, 1998: 376; isolectotypes CAL, K). 

106.

=Helosciadium pubescens DC., 1830, Prodr. 4:

Type: INDIA. "Ad Kamoon Indiae orientalis, 1829, Wallich [567]" (lectotype G-DC! - barcode P00753112, designated here; isolectotypes BM! barcode BM000622362, CAL, G! - barcode G00358202, K-WALLICH,! LE!, P!).

$=$ Helosciadium trifoliatum DC., 1830, Prodr. 4: 106.

= Pimpinella diversifolia DC. var. trifoliata (DC.) Kuntze, 1891, Revis. Gen. Pl. 1: 269.

Type: NEPAL. "Nepaliae montibus excelsis et circa Gossainthan, Wallich [565]" (lectotype G-DCbarcode G00358201, designated here; isolectotypes $\mathrm{K}$ !, LE!, WU!).

= Platyrhaphe japonica Miq., 1867, Ann. Mus. Bot. Lugduno-Batavi 3: 56. 1867; Prolus. Fl. Jap.: 244.

Type: JAPAN. "In valle montis Mamiwara in prov. Higo, Siebold (lectotype L! - barcode L0008386, designated here; isolectotypes L! - barcodes L0008384, L0008385).

= Pimpinella sinica Hance, 1868, J. Bot. 6: 113 .

Type: CHINA. "Inter gramina, solo arenoso, ad angiportum Tsing-yune, fl. North River, prov. Cantonensis, 18. 09. 1866, Sampson et Hance 13739" (lectotype K! - barcode K000685543, designated here; isolectotypes FI!, LE!, P!); "In montium Pakwan supra Cantonem, 03. 09. 1869, Sampson 13739" (syntypes BM, FI!, K! - barcode K000685542, NY?, P!).

$=$ Pimpinella diversifolia DC. var. simplicifolia Kuntze, 1891, Revis. Gen. Pl. 1: 269.

Type: INDIA. "Sikkim, Tungloo $3000 \mathrm{~m}$, 11. 1875. Kuntze 6890" (holotype NY - barcode NY00406196).

= Pimpinella tonkinensis Cherm., 1922, Bull. Soc. Bot. France 68: 511.

Type: VIETNAM. "Tonkin: prov. De Lao-Kay, Cha-pa, 1400-1500 m, 06. 12. 1913, Chevalier 29430" (syntype P! - barcode P00752850); ibid. "06. 12. 1913, Chevalier 29406" (lectotype P! barcode P00752842, designated here); ibid., "Hautefeuille 55,113,125,187,189" (syntypes P! barcodes P00754845, P00754846, P00754847, P00754848, P00754849); ibid., "Lecompte, Finet 498, 544" (syntypes P! - barcode P00752843).

= Trachydium cambodgianum $(\mathrm{H}$. Boissieu) $\mathrm{M}$. Hiroe var. flotoi M. Hiroe, 1962, Dansk Bot. Ark. 20: 194.

Type: THAILAND. "Poo Kradeng. Open grassy pine forest. Alt. 1300 m, 12. 07. 1958, Floto 7549" (holotype KYO!; isotype C!).
= Aegopodium burttii Nasir, 1972, in Nasir \& Ali, Fl. W Pakistan 20: 68, fig. 20, A, B.

Type: PAKISTAN. "B-7 Tribal Hazara, Chial Sar to Ganja Kandao, $8500 \mathrm{ft}$. Burtt 1222" (holotype E - barcode E00000546).

Distribution in China: NW: Gansu; Tibet: Xizang A. R., Qinghai; N: Shaanxi, Hebei, Shandong; Centr.: Henan, Jiangsu, Anhui, Hubei, Hunan, Jiangxi, Zhejiang, Fujiang; SW: Sichuan, Guizhou, Yunnan; S: Taiwan, Guangxi Zhuang A.R., Guangdong, Hainan Dao.

Distribution outside China: Japan, Vietnam, Cambodia, Thailand, Myanmar, Bhutan, Nepal, India, Pakistan, Afghanistan.

Pimpinella diversifolia DC. var. angustipetala Shan Renhwa et Pu Fating, 1983, Acta Phytotax. Sin. 21(1): 81.

Type: CHINA. "Sichuan: Omei Shan, Ley 005" (holotype NAS).

Distribution in China: N: Shanxi; SW: Sichuan. Endemic.

Pimpinella diversifolia DC. var. stolonifera Hand.-Mazz., 1933, Symb. Sin. 7(3): 714.

Type: CHINA. "NW Yunnan: Im Regenmischwalde und Hochstaudenfluren des birm. Mons. zwischen Mekong und Salwin unter dem Schondsula gegen Londjre, 22. 09. 1915, Handel-Mazzetti 8283" (holotype WU! - barcode WU0025989).

Distribution in China: SW: Yunnan. India.

Distribution outside China: Bhutan, Nepal,

Pimpinella duclouxii H. Boissieu, 1909, Bull. Soc. Bot. France 56: 351.

Type: CHINA. "Yun-nan, Lou-pou, près de Tong-Tchouen, 01. 09. 1907, Ducloux 5404" (holotype P! - barcode P00753111).

Distribution in China: SW: Yunnan. Endemic.

Pimpinella fargesii H. Boissieu, 1902, Bull. Herb. Boissier (2 sér.) 2: 808.

Type: CHINA. "Su-Tchuen oriental, district Tchen-Kéou-Tin, Farges" (lectotype P! - barcode P00834788, designated here; isolectotypes B! barcode B 10 0367218, K! - barcode K000685540, L!, P! - barcodes P00834789, P00834790, PE!); "Prov. Szechwan/S.Wushian, 03. 1889, Henry 7331" (syntype P! - barcode P00834792).

= Pimpinella fargesii $\mathrm{H}$. Boissieu var. alba $\mathrm{H}$. Boissieu, 1909, Bull. Soc. Bot. France 56: 350.

Type: CHINA. "Su-Tchuen oriental a TchenKéou-Tin, Farges" (holotype P!). 
Distribution in China: Centr.: Anhui, Hubei, Jiangxi; SW: Sichuan. Endemic.

Pimpinella filipedicellata Liou Shoulu, 1990, Acta Phytotax. Sin. 28(2): 145.

Type: CHINA. "Xizang: Cui-Ji-la-shan, 06. 09. 1952, Tsoong Puchin 5337" (holotype PE).

Distribution in China: Tibet: Xizang A. R. Endemic.

Pimpinella flaccida C. B. Clarke, 1889, J. Linn. Soc. Bot. 25: 28, tab. 15.

$\equiv$ Carum flaccidum (C. B. Clarke) Franch., 1894, Bull. Annuel Soc. Philom. Paris (sér. 8) 6: 126.

Type: INDIA. "Kohima, alt. 5300 feet [Nagaland], 1885, Clarke 41139" (holotype K! barcode K000685495).

= Pimpinella refracta $\mathrm{H}$. Wolff, 1929, Repert. Spec. Nov. Regni Veg. 27: 190.

Type: CHINA. "Yunnan, West China: Banks of the Mekong. 6000'. Lat. 28 $12^{\circ}$ ', shady thicket, 09. 1917, Forrest 14885" (holotype E! - barcode E00000497).

Distribution in China: SW: Sichuan, Guizhou, Yunnan.

Distribution outside China: India.

Pimpinella grisea H. Wolff, 1929, Repert. Spec. Nov. Regni Veg. 27: 184.

Type: CHINA. "Tibet: A-tun-tsi, 12-13000' s.m., 01. 08. 1913, Kingdon-Ward 924" (holotype $\mathrm{E}$ !).

Distribution in China: Tibet: Xizang A. R.; SW: Yunnan. Endemic.

Pimpinella heliosciadea H. Boissieu, 1902, Bull. Herb. Boissier (2 sér.) 2: 809.

Type: CHINA. "Su-Tchuen oriental, district Tchen-Kéou-Tin, Farges" (holotype P! - barcode P00753107).

= Pimpinella smithii $\mathrm{H}$. Wolff, 1926, Acta Horti Gothob. 2: 307.

Type: CHINA. "Sze-ch'uan bor.: Sung-pan, gegen Westen, auf üppigen Buschwiesen, 3100 $\mathrm{m}$ [In prato herboso-fruticoso], 31. 07. 1922, H. Smith 3556" (lectotype UPS!, designated here; isolectotype GB! - barcode GB-0048819).

= Pimpinella stricta H. Wolff, 1926, Acta Horti Gothob. 2: 308.

Type: CHINA. "Sze-ch'uan bor-occid.: Drogochi, 3500 m, 26. 09. 1922, H. Smith 4676" (holotype UPS!).

Distribution in China: NW: Gansu; Tibet: Xizang A. R., Qinghai; N: Neimenggu A. R.,
Shaanxi, Shanxi, Hebei; Centr.: Henan, Anhui, Hubei; SW: Sichuan, Yunnan; S: Guangxi Zhuang A. R. Endemic.

Pimpinella henryi Diels, 1900, Bot. Jahrb. Syst. 29 (3-4) (Fl. Centr. China): 495.

$\equiv$ Pimpinella sutchuensis H. Boissieu, 1902, Bull. Herb. Boissier (2 sér.) 2: 808.

Type: CHINA. "Ichang, prov. Hupeh, 18851888, Henry 7101" (lectotype E! - barcode E00000474, designated here; isolectotypes E!, K barcode K000685530), P! - barcode P00602139, US!); "Prov. Szechuan (N Wushan), 03. 1889, Henry 7101" (syntype K!); "Nan ch'uan: K'u leitzu pa, Berghänge, Rosthorn 1033" (syntype); "Setchuen: Nan ch'uan, Fang shui pa, Hochwald, 17. 09. 1891. Rosthorn 942" (syntype O!).

Distribution in China: NW: Gansu; N: Shaanxi, Shanxi; Centr.: Hubei; SW: Sichuan. Endemic.

Pimpinella kingdon-wardii H. Wolff, 1929, Repert. Spec. Nov. Regni Veg. 27: 184.

Type: CHINA. "Tibet, Doker-la. Forest undergrowth., 10-11000 ft., Kingdon-Ward 1100" (holotype E!).

$=$ Pimpinella weishanensis Shan Renhwa et $\mathrm{Pu}$ Fating, 1983, Acta Phytotax. Sin. 21(1): 79, tab. 1, fig. 6-10.

Type: CHINA. "Yunnan: Weishan, alt. 2300 $\mathrm{m}$, in open ravine, 08. 09. 1933, Tsiang 11891" (holotype NAS; isotype KUN!).

- Pimpinella monoica auct. non Dalzell: Shan Renhwa, 1940, Sinensia 11(1-2): 170.

Distribution in China: Tibet: Xizang A. R.; SW: Sichuan, Yunnan. Endemic. 122.

Pimpinella leschenaultii DC., 1830, Prodr. 4:

Type: INDIA. "In Indiae orientalis montibus Nellygerry [Nilghiri], Leschenault 180" (holotype P! - barcode P00753101).

= Carum candolleanum (Wight et Arn.) Franch., 1894, Bull. Annuel Soc. Philom. Paris (sér. 8) 6: 128, non Pimpinella candolleana Wight et Arn.

= Pimpinella pulneyensis Gamble, 1919, Bull. Misc. Inform. Kew: 228.

Type: INDIA. "Pulney Hills, Bear Shola stream, Kodaikanal, Tamil Nadu, 24. 06. 1898, Bourne" (lectotype K! - barcode K000685490, designated here; isolectotype CAL).

Distribution in China: SW: Sichuan, Guizhou, Yunnan; S: Guangxi Zhuang A. R., Guangdong.

Distribution outside China: Vietnam, India, Sri-Lanka. 
Pimpinella liiana M. Hiroe, 1958, Umbell. Asia 1: 60 .

Type: CHINA. "Prov. Yunnan: Grassy mountain slope, Bon-mai, alt. ca. 2400 m, Chingtung, 21. 12. 1939, M. K. Li 2568" (holotype UC).

Distribution in China: SW: Yunnan. Endemic.

Pimpinella limprichtii H. Wolff, 1922, Repert. Spec. Nov. Regni Veg. Beih. 12 (Limpr. Bot. Reise China Ost-Tibet): 450.

Type: CHINA. "Tschili: Jehol, auf dem Wu ling schan, im Bannwalde der östlichen Kaisergraber, 2000 m, Limpricht 2930".

Distribution in China: N: Hebei. Endemic.

Pimpinella niitakayamensis Hayata, 1917, Gen. Ind. Fl. Formos.: 33; 1921, Icon. Pl. Formosan. 10: 20, fig. 11.

Type: CHINA. "[Taiwan] Monte Morrisson, ad 13000 ped. alt., 20. 10. 1906, Kawakami, Mori" (holotype TAIF).

= Pimpinella astilbifolia Hayata, 1921, Icon. P1. Formosan. 10: 20, fig. 10.

Type: CHINA. "[Taiwan] Niitakayama, Nokozan, Nishigundaizan, Matsuda" (syntype TAIF).

Distribution in China: S: Taiwan. Endemic.

Pimpinella nyingchiensis Pan Zehui et Yao Gan, 1992, Acta Phytotax. Sin. 30(3): 263, fig. 1.

Type: CHINA. "Xizang: Nyingchi, alt. 3150 m, under forests, 22. 08. 1989, Yao Gan et al. 2117" (holotype NAS).

Distribution in China: Tibet: Xizang A. R. Endemic.

Pimpinella pimpinellisimulacrum (Farille et $\mathrm{S}$. B. Malla) Farille, 1985, Candollea 40(2): 554.

$\equiv$ Similisinocarum pimpinellisimulacrum Farille et S. B. Malla, 1984, Bull. Soc. Bot. France, Lett. Bot. 131(1): 70.

Type: NEPAL. "Haute vallée de la Kali Gandaki, spigolo au SEE de Muktinath (Muktinath-Range, à nord de l'Annapurna), dans la lande à Rhododendron chamephytiques, alt. 4500 m, 18. 09. 1981, Farille 81422 (holotype KATH; isotypes BM, P?).

Distribution in China: Tibet: Xizang A. R.

Distribution outside China: Nepal.

Pimpinella puberula (DC.) Boiss., 1844, Ann. Sci. Nat. Paris (sér. 3, Bot.) 1 (Pl. Aucher.): 129.

EPtychotis puberula DC., 1830, Prodr. 4: 109.

Type: IRAQ. "In Oriente circa Baghdad [de Bagdad à Aleppo; de Baghdad à Kermansha],
Olivier, Bruguière" (lectotype G-DC!, designated here; isolectotype P! - barcodes P00752874, P00752875, P00752882).

= Pimpinella petraea Nábělek, 1923, Spisy Prir. Fak. Masarikovy Univ. 35: 122, tab. 13, fig. 2., nom. illeg. (Art. 53.1), non Willd. ex Spreng. (1818).

= Pimpinella cretica Poir. var. petraea Zohary, 1972, Fl. Palaestina 2: 427.

= Pimpinella nabelekii M. Hiroe, 1979, Umbelliferae World: 834.

Type: EGYPT, SINAI. "In Arabia Petraea in valle Seil ed Dra inter El-Kerak et Et-Tafile, alt. ca. 500 m, 15. 06. 1909, Nábělek 441" (lectotype SAV! barcode SAV0002324; isolectotype SAV - barcodes SAV0002322, SAV0002323, SAV0002325).

= Pimpinella cretica Poir. var. lasiopetala Feinbr., 1931, Repert. Spec. Nov. Regni Veg. 29: 134.

Type: JORDAN. "Mejdel (Gilead), 09. 05. 1927, Eig, Feinbrun, Zohary" (syntype); "Umgebung von Amman, 08. 05. 1927, Eig, Feinbrun, Zohary" (syntype); "Zwischen Jordan und Es-Salt, 06. 05. 1927, Eig, Feinbrun, Zohary" (syntype); "Callirrhoe, 03. 04. 1925, Eig, Feinbrun, Zohary” (syntype).

Distribution in China: NW: Xinjiang Weiwuer A. R.

Distribution outside China: Pakistan, Kazakhstan, Kirghizia, Tajikistan, Uzbekistan, Turkmenistan, Afghanistan, Iran, Azerbaijan, Turkey, Iraq, Kuwait, Qatar, Saudi Arabia, UAE, Yemen, Syria, Israel, Jordan.

Pimpinella purpurea (Franch.) H. Boissieu, 1906, Bull. Soc. Bot. France 53: 428.

$\equiv$ Carum purpureum Franch., 1894, Bull. Annuel Soc. Philom. Paris (sér. 8) 6: 127.

Type: CHINA. "Prov. du Yunnan, m. Tsang-chan, 2500 m, 05. 08. 1884, Delavay 673" (lectotype P! barcode P02284790, designated here; isolectotypes K!, NY - barcode NY00405780, P! - barcodes P02284791, P02284792, P02284793); "Ma-eulchan, in silvis supra Gnou-kay, alt. 2500-3000 m, 06. 08. 1889, Delavay 3850" (syntypes P! - barcodes $\mathrm{P} 02519825, \mathrm{P} 02519827, \mathrm{P} 02519830, \mathrm{P} 02519831$, $\mathrm{P} 02519832, \mathrm{P} 02519834)$.

= Pimpinella markgrafiana Fedde ex H. Wolff, 1929, Repert. Spec. Nov. Regni Veg. 27: 189.

Type: CHINA. "Yangtze watershed, prefectural district of Likiang, eastern slopes of Likiang Snow Range, 10. 07. 1922, Rock 5005a" (syntype E!); "Yangtze watershed, prefectural district of Likiang, eastern slopes of Mt. Dyinaloko, northern peak of the Likiang Snow Range, 3962 m, 10. 07. 1922, Rock 10434" (syntype E! - barcode E00000484, US!). 
Distribution in China: SW: Yunnan.

Distribution outside China: Myanmar.

Pimpinella renifolia $\mathrm{H}$. Wolff, 1929, Repert. Spec. Nov. Regni Veg. 27: 191.

Type: CHINA. "Hupeh: Ichang and immediate neighbourhood, 02. 1887, Henry 2923" (holotype K! - barcode K000685524).

Distribution in China: Centr.: Hubei. Endemic.

Pimpinella rhomboidea Diels, 1900, Bot. Jahrb. Syst. 29 (3-4) (Fl. Centr. China): 496.

Type: CHINA. "Setchuen, Nan ch'uan: Kufu tung, 16. 09. 1891, Rosthorn 930" (holotype O!).

Distribution in China: NW: Gansu; N: Shaanxi, Hebei; Centr.: Henan, Hubei; SW: Sichuan, Guizhou. Endemic.

Pimpinella rhomboidea Diels var. tenuiloba Shan Renhwa et Pu Fating, 1989, Acta Phytotax. Sin. 27(1): 63, tab. 2.

Type: CHINA. "Sichuan: Lixian, alt. 3400 m, in alpine meadows, 03. 09. 1983, Lixian Medic. Herb. Exped. 20800" (holotype CDBI!).

Distribution in China: SW: Sichuan. Endemic.

Pimpinella rubescens (Franch.) H. Wolff, 1933, in Hand.-Mazz., Symb. Sin. 7(3): 715.

$\equiv$ Hydrocotyle rubescens Franch., 1894, Bull. Annuel Soc. Philom. Paris (sér. 8) 6: 108.

$\equiv$ Centella rubescens (Franch.) Domin, 1908, Bot. Jahrb. Syst. 41(3): 157.

Type: CHINA. "Yunnan, in umbrosis humidis ad Likiang, haud procul a nivibus perpetuis, alt. 3500 $\mathrm{m}$ [Lieux ombrages et un peu humides, glacier de Li Kiang, a 3500 mètres d'altitude], 09. 07. 1884, Delavay 181" (lectotype P! - barcode P00752874, designated here; isolectotype P! - barcode P00752873); "Yunnan, ad collum Yen-tze-hay, alt. $3200 \mathrm{~m}$ [Lieux ombrages et humides a $3200 \mathrm{~m}$ d'altid., col de Yen tze hay], 18. 07. 1887, Delavay" (syntypes K! - barcode K000685522, P! - barcodes P000685522, P02519798, P02519799, P02519800).

Distribution in China: SW: Sichuan, Yunnan. Endemic.

Pimpinella scaberula (Franch.) H. Boissieu, 1906, Bull. Soc. Bot. France 53: 428.

$\equiv$ Carum scaberulum Franch., 1894, Bull. Annuel Soc. Philom. Paris (sér. 8) 6: 125.

$\equiv$ Trachyspermum scaberulum (Franch.) H. Wolff, 1933, in Hand.-Mazz., Symb. Sin. 7(3): 713.

Type: CHINA. "Yun-nan, in silvis ad fauces Santchang-kiou supra Ho kin, 03. 09. 1887, Delavay
2716" (lectotype P!-barcodeP00752746, designated here; isolectotypes K!, NY - barcode NY00405781, P! - barcodes P01818126, P01818127); "Les gorges de Tchong Kiou, 11. 09. 1889, Delavay 3908" (syntype P! - barcode P00752748); "Yun-nan, in sterilibus inter Tcheou Choui et Ta-long-tan, alt. $1800 \mathrm{~m}$, 13-22. 09. 1887, Delavay 2736" (syntypes G, P! barcodes P00752747, P00752869, P00752870).

= Trachyspermum triradiatum $\mathrm{H}$. Wolff, 1926. Acta Horti Gothob. 2: 305.

Type: CHINA. "Sze-ch'uan bor-occid.: Drogochi, auf trockenen, sonnigen, bebuschten Hügeln, 3200 m. In collo aprico, 25. 09. 1922, H. Smith 4489" (holotype UPS!).

Distribution in China: SW: Sichuan, Guizhou, Yunnan; S: Guangxi Zhuang A. R. Endemic.

Pimpinella scaberula (Franch.) H. Boissieu var. ambrosiifolia (Franch.) H. Wolff, 1927, in Engler (ed.), Pflanzenreich 90(IV, 228): 274.

ECarum scaberulum Franch. var. ambrosiifolium Franch., 1894, Bull. Annuel Soc. Philom. Paris (sér. 8) 6: 126.

$\equiv$ Trachyspermum scaberulum (Franch.) H. Wolff var. ambrosiifolium (Franch.) Shan Renhwa, 1940, Sinensia 11(1-2): 166.

Type: CHINA. "Yun-nan, inter Tcheou-Choui et Ta-long, 16. 08. 1886, Delavay 3115" (syntypes P! - barcodes P00854705, P00854706, P02519888, P02519890, P02519891, K!); "Yun-nan, in pascuis prope Pe-cha-kay, haud procul a Likiang, 16. 08. 1886, Delavay 2457" (syntypes P! - barcodes P02519882, P02519884, P02519885, P00834704, P02519887, NY - barcode NY00405782).

Distribution in China: SW: Yunnan. Endemic.

Pimpinella serra Franch. et Sav., 1879, Enum. P1. Jap. 2: 372.

三 Sium serrum (Franch. et Sav.) Kitag., 1941, J. Jap. Bot. 17: 562.

Type: JAPAN. "Nikko, Prov. Shimotsuke, Savatier 515" (holotype P! - barcode P00752868).

= Sium ternifolium Lee Byoung Youn et Ko Sung Chul, 2009, Korean J. Pl. Taxon. 39(3): 130, fig. $1-2$.

Type: KOREA. "Gangwon-do Province, Wonjusi city, understory in the forests of Carpinus laxiflora and Betula chinensis, near stream, alt. 480-495 m, 20. 08. 2009, Ko 064185" (holotype HNHM; isotypes $\mathrm{HNHM}, \mathrm{KB}, \mathrm{NH}$ ).

Distribution in China: Centr.: Anhui.

Distribution outside China: Japan, Korea. 
Pimpinella silvatica Hand.-Mazz., 1933, Symb. Sin. 7(3): 714.

Type: CHINA. "Prov. Setschwan austro-occid.: in districtus monasterii Muli in septentr. pagi Yunnanensis Yungning regione temperatae, in silva loco Gumadi dicto, 04. 08. 1915, Handel-Mazzetti 7439" (holotype WU! - barcode WU0025946).

Distribution in China: SW: Sichuan, Yunnan. Endemic.

Pimpinella souliei H. Boissieu, 1902, Bull. Herb. Boissier (2 sér.) 2: 810 .

= Tongoloa souliei $($ H. Boissieu) H. Wolff, 1927, in Engler (ed.), Pflanzenreich 90(IV, 228): 319.

Type: CHINA. "Su-Tchuen oriental a Tongolo [Thibet oriental, principauté du Kia-la (Ta-tsienlou), Tongolo], 06. 1892, Soulié 464" (lectotype P! barcode P00752876, designated here; isolectotype $\mathrm{P}$ !).

Distribution in China: SW: Sichuan. Endemic.

Note: Probably the species could be transferred to Trachyspermum.

Pimpinella tagawae M. Hiroe, 1958, Umbell. Asia 1: 61.

Type: CHINA. "Formosa, prov. Taihoku (Taipeh): Rato-gun, inter Ekizyu-kei et Piyanananbu, alt. 1000-2000 m, 27. 07. 1934, Tagawa 105" (holotype KYO!).

Distribution in China: S: Taiwan. Endemic.

Pimpinella thellungiana H. Wolff, 1927, in Engler (ed.), Pflanzenreich 90(IV, 228): 304.

Type: CHINA. "Sud-Shansi, Yuan-chu District, auf Wiesen $2800 \mathrm{~m}$ u. M., bluhend im Juli, H. Smith 6395" (syntype); "Zentral-Shansi, Chiaoching-District, 2100-2300 m u. M., H. Smith 623" (syntype); "Mandschurei, Amurgebiet, Komarov 1127" (syntype LE?). RUSSIA. "Dahuria, Turczaninow" (syntype LE?). JAPAN. "Japonia, Kundsso-san, in pratis alpinis. 29.09-11. 10. 1863. Maximowicz" (syntype LE!).

Distribution in China: N: Neimenggu A. R., Shaanxi, Shanxi, Hebei, Shandong; NE: Heilongjiang, Jilin, Liaoning; Centr.: Henan, Hubei.

Distribution outside China: Russia, Mongolia.

Pimpinella thyrsiflora H. Wolff, 1930, Repert. Spec. Nov. Regni Veg. 27: 320.

Type: CHINA. "Yunnan, Mengtze, Mts to N, 7000', Henry 10225" (lectotype E! - barcode E00000477, designated here; isolectotype LE!).

Distribution in China: SW: Yunnan. Endemic.
Pimpinella tibetanica H. Wolff, 1930, Repert. Spec. Nov. Regni Veg. 27: 319.

Type: CHINA. "Tibet: Yatung 27 $51^{\prime}$ Lat., $38^{\circ} 35^{\prime}$ Long. or., 1897, Hobson" (holotype K! barcode K000685471).

Distribution in China: Tibet: Xizang A. R.; SW: Sichuan, Yunnan.

Distribution outside China: Bhutan, Nepal, India.

Pimpinella triternata Diels, 1900, Bot. Jahrb. Syst. 29 (3-4) (Fl. Centr. China): 496.

Type: CHINA. "Central-China: Sze-ch'uan, Nan ch'uan: Ton shihai, Wiesen [Setchuen, Nan ch'uan, Ton shin ai. 30. 09. 1891, von Rosthorn 1070" (holotype O!; photo LE).

Distribution in China: SW: Sichuan. Endemic.

Pimpinella urbaniana Fedde ex H. Wolff, 1930, Repert. Spec. Nov. Regni Veg. 27: 330.

Type: CHINA. "Yunnan: Lou pou près Tongtchouan, 07. 1909, Ducloux 6492" (holotype P! barcode P00752858).

Distribution in China: SW: Yunnan. Endemic.

Pimpinella valleculosa $\mathrm{Fu}$ Kuntsun, 1981, Fl. Tsinling. 1(3): 457, 405, fig. 348.

Type: CHINA. "Kansu: Wen Hsien, prope Pikow, alt. 620 m, 20. 10. 1974, Fu Kuntsun 17287" (holotype WUK).

Distribution in China: NW: Gansu; N: Shaanxi; Centr.: Hubei; SW: Sichuan. Endemic.

Pimpinella xizangensis Shan Renhwa et $\mathrm{Pu}$ Fating, 1986, Acta Phytotax. Sin. 24(4): 311, fig. 7.

Type: CHINA. "Xizang: Lhunze, alt. $2700 \mathrm{~m}$, 16. 07. 1975, Exped. Qinghai-Xizang 750624" (holotype PE; isotype KUN!).

Distribution in China: Tibet: Xizang A. R. Endemic.

Pimpinella yunnanensis (Franch.) H. Wolff, 1927, in Engler (ed.), Pflanzenreich 90(IV, 228): 266.

$\equiv$ Carum yunnanense Franch., 1894, Bull. Annuel Soc. Philom. Paris (sér. 8) 6: 128.

Type: CHINA. "Yun-nan, in pratis humidis supra Sin-tsen, prope Mo-so-yn, 14. 09. 1889, Delavay 3903" (lectotype P!, designated here; isolectotypes K! - barcode K000685538, NY barcode NY00405783).

Distribution in China: SW: Sichuan, Yunnan. Endemic. 
Pleurospermum albimarginatum H. Wolff, 1925, Repert. Spec. Nov. Regni Veg. 21: 243.

Type: CHINA. "Tibetia orientalis: Tongolo, 06. 1894, Soulié 2618”' (holotype P?).

Distribution in China: SW: Sichuan. Endemic.

Pleurospermum longipetiolatum H. Wolff, 1925, Repert. Spec. Nov. Regni Veg. 21: 242.

Type: CHINA. "Tibetia orientalis: Ta-tsien-lu, 1894, Soulié 2178" (holotype P?).

Distribution in China: SW: Sichuan. Endemic.

Pleurospermum microphyllum H. Wolff, 1925, Repert. Spec. Nov. Regni Veg. 21: 242.

Type: CHINA. "Tibetia orientalis: Tongolo, Soulié 2605" (holotype P?).

Distribution in China: SW: Sichuan. Endemic.

Pleurospermum microsciadium H. Wolff, 1925, Repert. Spec. Nov. Regni Veg. 21: 241.

Type: CHINA. "Tibetia orientalis: Tongolo: Tizu, 07. 1894, Soulié 2614" (holotype P?).

Distribution in China: SW: Sichuan. Endemic.

Pleurospermum souliei H. Wolff, 1924, Repert. Spec. Nov. Regni Veg. 19: 309.

Type: CHINA. "Tibetia orientalis: Tatsien-lu, Soulié 2186" (holotype P?).

Distribution in China: SW: Sichuan. Endemic.

Pleurospermum tripartitum Pu Fating, Li Rong et Li Heng, 2012, Nordic J. Bot. 30: 178.

Type: CHINA. "Yunnan Province, Fugong Xian, Lumadeng Xiang, Yaping Cun, below Amero Pass along the road back down to the confluence of the north and south fork of Yamu He, east side of Gaoligong Shan, coniferous forest, Abies forest with Rhododendron and bamboo thickets, growing along stream in sun, $27^{\circ} 4^{\prime} 49.5^{\prime \prime} \mathrm{N}, 98^{\circ} 44^{\prime} 52.2^{\prime \prime} \mathrm{E}, 3120$ $\mathrm{m}$ a.s.1., 13. 08. 2005, Gaoligong Shan Biodiversity Survey 27178" (holotype KUN; isotypes CDBI, $\mathrm{GH}$ - barcode GH 00353471).

Distribution in China: SW: Yunnan. Endemic.

Pleurospermum uralense Hoffm., 1814, Gen. Pl. Umbell.: IX, tab. 1B, 2.

三Pleurospermum austriacum (L.) Hoffm. subsp. uralense (Hoffm.) Horn, 1946, Svensk Bot. Tidskr. 40: 182. 1.

Type: RUSSIA. "Hortum Gorenk. et botanicum Mosquens introducto F.G.Helm."

= Pleurospermum camtschaticum Hoffm., 1814, Gen. pl. Umbell.: X.
= Ligusticum camtschaticum (Hoffm.) Steud., 1821, Nomencl. bot.: 478.

Type: RUSSIA. "Kamtschatka, Langsdorff" (holotype LE!).

= Laserpitium athamantae Spreng., 1820, in Roemer \& Schultes (eds.), Syst. Veg. (ed. 15 bis) 6: 624.

Type: RUSSIA. "In Sibiria".

= Pleurospermum archangelica Ledeb., 1829 ., F1. Altaic. 1: 369.

Type: KAZAKHSTAN. "In Sibiria altaica ad rivulorum margines circa Riddersk".

- Pleurospermum austriacum auct. non Hoffm.: Ledeb., 1829, Fl. Altaic. 1: 368.

Distribution in China: NW: Xinjiang Weiwuer A. R.; N: Neimenggu A. R., Shaanxi, Shanxi, Hebei; NE: Heilongjiang, Jilin, Liaoning; Centr.: Henan.

Distribution outside China: Russia, Japan, Korea, Mongolia, Kazakhstan. Europe.

Prangos cachroides (Schrenk) Pimenov et V. N. Tikhom., 1981, in Czerepanov (ed.), Sosud. Rast. SSSR: 28 .

$\equiv$ Cryptodiscus cachroides Schrenk, 1841, Enum. P1. Nov. 1: 65.

ECachrys cryptodiscus Koso-Pol., 1916, Bull. Soc. Imp. Naturalistes Moscou, s.n. 29: 163, nom. illeg. (Art. 52.1).

三Neocryptodiscus cachroides (Schrenk) V. M. Vinogr., 1994, in Grubov (ed.), Rast. Tsentral'noi Azii 10: 59.

Type: KAZAKHSTAN. "Semina matura ad lacum Alakul lecta. 13. 07. 1840 [In collibus sabulosis Songoriae, inter fontem Sassykpastau et montes Arganaty, 1841], Schrenk" (holotype LE).

= Cryptodiscus rutifolius Bunge, 1851, Beitr. Fl. Russl. (A1. Lehmann Reliq. Bot.): 139.

= Cachrys rutifolia (Bunge) Koso-Pol., 1851, Bull. Soc. Imp. Naturalistes Moscou, s.n. 29: 163.

Type: KAZAKHSTAN. "Auf der Lehmsteppe zwischen dem Kuwan- und Syr-Darja. 09. 05. 1842, Lehmann" (syntype); "Häufig im Sande bei Malibasch am Syr-Darja, 13. 05. 1842, Lehmann 566" (lectotype LE!, designated by Vinogradova, 1999: 84); "Zwischen Malibasch und dem See Aigirak und auf Lehmflächen und Sandhügeln am Syr-Darja und Aigirak-See, 15. 05. 1842, Lehmann" (syntype).

Distribution in China: NW: Xinjiang Weiwuer A. R.

Distribution outside China: Russia, Kazakhstan, Kirghizia, Uzbekistan, Turkmenistan. 
Prangos didyma (Regel) Pimenov et V. N. Tikhom., 1981, in Czerepanov (ed.), Sosud. Rast. SSSR: 28.

$\equiv$ Cachrys didyma Regel, 1878, Trudy Imp. S.Peterburgsk. Bot. Sada 5(2): 601.

$\equiv$ Cryptodiscus didymus (Regel) Korovin, 1924, Bull. Sredne-Asiatsk. Gosud. Univ. 7, Suppl. (Sched. Herb. Fl. As. Med. 1-2): 23.

$\equiv$ Neocryptodiscus didymus (Regel) Hedge et Lamond, 1987, in Rechinger (ed.), Fl. Iranica 162: 208.

Type: TAJIKISTAN. "In montibus karatavicis Mogol-tau, 20. 04. 1868, Sewerzov" (lectotype LE!, designated by Geldykhanov, 1992: 104).

$=$ Cachrys didyma Regel var. glabra Regel, 1878, Trudy Imp. S.-Peterburgsk. Bot. Sada 5(2): 601.

Type: KAZAKHSTAN. "Desertum Aralense. Regio fluv. Ssyr-Darja, inter Murtur et Kara-bogut, 14. 05. 1858, Borszczow 729" (lectotype LE!, designated here; isolectotype LE!).

Distribution in China: NW: Xinjiang Weiwuer A. R.

Distribution outside China: Kazakhstan, Kirghizia, Tajikistan, Uzbekistan, Turkmenistan, Afghanistan.

Note: Closely related and probably identical with the previous species.

Prangos herderi (Regel) Herrnst. et Heyn, 1977, Boissiera 26: 63, fig. 17.

$\equiv$ Cachrys herderi Regel, 1877, Trudy Imp. S.Peterburgsk. Bot. Sada 5(2): 601.

Type: KAZAKHSTAN. "In Turkestaniae orientalis montibus alatavicis cis-iliensibus Kara Tschek, Semenov" (holotype LE!). A. R.

Distribution in China: NW: Xinjiang Weiwuer

Distribution outside China: Kazakhstan.

Prangos herderi (Regel) Herrnst. et Heyn subsp. xinjiangensis Chen Xiaoya et Liu Qixing, 1989, Bull. Bot. Res., Harbin 9(3): 99, fig. 1-5.

Type: CHINA. "Xinjiang, Guozigou, Tianshan Mt., alt. 1100 m, 13. 06. 1987, Chen Xiaoya, Liu Qixing 87366" (holotype NAS).

Distribution in China: NW: Xinjiang Weiwuer A. R. Endemic.

Prangos ledebourii Herrnst. et Heyn, 1977, Boissiera 26: 68, fig. 20.

$\equiv$ Cachrys macrocarpa Ledeb., 1829, Fl. Altaic. 1: 364 .

Type: KAZAKHSTAN. "In collibus apricis et rupestribus deserti songoro-kirghisici ex adverso fortalitii Ustkamenogorsk et alibi, Ledebour" (syntypes HAL?, LE); "Inter fortalitium Buchtorminsk et lacum, qui Noor-Saisan vocatur [Altai. In campestribus et collibus apricis petrosis a fortalitio Buchtarminsk orientem versus usque ad montes Dolen-Kara et Arkaul], 1826, Meyer 228" (lectotype LE!, designated by Vinogradova, 1999: 83).

Distribution in China: NW: Xinjiang Weiwuer A. R.

Distribution outside China: Mongolia, Kazakhstan.

Pternopetalum bipinnatum Wang Lisong, 2005, Bot. Zhurn. (St. Perersburg) 90(12): 1898.

Type: CHINA. "China, Guangxi Province, Leye County, Tonglezheng, Yangling, alt. $900 \mathrm{~m}$, on brushy limestome slope, 07. 05. 1989, Plant Expedition of upper reaches in Hong-shui River 89-1019" (holotype KUN).

Distribution in China: S: Guangxi Zhuang A. R. Endemic.

Pternopetalum botrychioides (Dunn) Hand.Mazz., 1933, Symb. Sin. 7(3): 718.

$\equiv$ Cryptotaeniopsis botrychioides Dunn, 1903, J. Linn. Soc. Bot. 35: 494.

Type: CHINA. "West Szechuen, Tachienlu, at 9000-13500 ft., Pratt 839" (lectotype K - barcode K000913311, designated by Wang Lisong, 2008: 289; isolectotype P! - barcode P00432442); "Mt. Omei at $8000 \mathrm{ft}$, Faber 629" (syntype).

= Carum delicatulum H. Wolff, 1922, Repert. Spec. Nov. Regni Veg. Beih. 12 (Limpr. Bot. Reise China Ost-Tibet): 449 ..

$=$ Cryptotaeniopsis delicatula $(\mathrm{H}$. Wolff $) \mathrm{H}$. Wolff, 1926, Acta Horti Gothob. 2: 306.

= Pternopetalum delicatulum (H. Wolff) Hand.Mazz., 1933, Symb. Sin. 7(3): 718.

Type: CHINA. "Szetchuan: Kianting fu, Omi schan. Felsen, 3000 m, Limpricht 1498" (holotype WU! - barcode WU0025968).

$=$ Cryptotaeniopsis affinis H. Wolff, 1930, Repert. Spec. Nov. Regni Veg. 27: 328.

$=$ Pternopetalum affine (H. Wolff) M. Hiroe, 1979, Umbelliferae World: 1013, "affinum".

Type: CHINA. "Yunnan: Vallon de Ku-longtchang, alt. $800 \mathrm{~m}$ s.l., Maire" (holotype P! - barcode P00752728).

=Pternopetalum botrychioides (Dunn) Hand.Mazz var. latipinnulatum Shan Renhwa, 1940, Sinensia 11(1-2): 158.

Type: CHINA. "Szechuan: Kuan Hsien, alt. 3000-3600 ft., 06. 07. 1928, W. P. Fang 2062". 
Distribution in China: SW: Sichuan, Guizhou, Yunnan. Endemic.

Pternopetalum caespitosum Shan Renhwa, 1943, Sinensia 14: 113, fig. 2.

Type: CHINA. "Sikang: Tan-pa Hsien, Mouniu, Cha-si-kou, on shady woods, 10. 08. 1940, K. L. Chu 7642B" (holotype NAS!).

Distribution in China: NW: Gansu; Tibet: Xizang A. R.; N: Shaanxi; SW: Sichuan. Endemic.

Pternopetalum cuneifolium (H. Wolff) Hand.Mazz., 1933, Symb. Sin. 7(3): 718.

三 Cryptotaeniopsis cuneifolia H. Wolff, 1929, Repert. Spec. Nov. Regni Veg. 27: 181.

Type: CHINA. "[Yunnan] Vallées-rives des torrents à Tien-sin, alt. 3000 m, 1914, Maire 620” (holotype E!).

= Pternopetalum cartilagineum Wu Zhengyi, 1978, Acta Phytotax. Sin. 16(3): 70, fig. 1.

Type: CHINA. "Yunnan, Zhengkang [Yongde] Xian, alt. 2500 m, oak forest, along river, 03. 1936, C. W. Wang 72593" (holotype KUN; isotypes NAS, $\mathrm{PE}$ !).

= Pternopetalum molle (Franch.) Hand.-Mazz. var. crenulatum Shan Renhwa et Pu Fating, 1978, Acta Phytotax. Sin. 16(3): 72.

Type: CHINA. "Yunnan, H. T. Tsai 55935A" (lectotype IBSC, designated by Wang Lisong (2008: 285); isolectotypes GH! - barcode GH00077635, NAS, PE!, SZ).

Distribution in China: SW: Yunnan. Endemic.

Pternopetalum davidii Franch., 1888, Nuov. Arch. Mus. Hist. Nat. (sér. 2) 10 (P1. David. 2): 64 (246). "davidi".

E Cryptotaeniopsis davidii (Franch.) H. Wolff, 1927, in Engler (ed.), Pflanzenreich 90(IV, 228): 175.

Type: CHINA. "Moupine, in montibus editissimis, secus rivulos, 04. 1869, David" (lectotype P! - barcode P00492877, designated here; isolectotypes $\mathrm{K}$ ! - barcode K000685614, P! barcodes P00492875, P00492876).

Distribution in China: NW: Gansu; Tibet: Xizang A. R.; N: Shaanxi; Centr.: Hubei; SW: Sichuan, Guizhou, Yunnan. Endemic.

Pternopetalum delavayi (Franch.) Hand.-Mazz., 1933, Symb. Sin. 7(3): 718.

三 Carum delavayi Franch., 1894, Annuel Soc. Philom. Paris (sér. 8) 6: 120.

E Cryptotaeniopsis delavayi (Franch.) Dunn, 1903, J. Linn. Soc. Bot. 35: 495.
Type: CHINA. "Yunnan, in silvis montanis ad Yang in chan supra Lankong, 01. 08. 1883, Delavay 97" (lectotype P - barcode P00432463, designated by Wang Lisong, 2008a: 110; isolectotypes K, P barcode P00432464); "Yunnan, in sylvis ad faucem San-tschang-kiou, prope Hokin, alt. 2500 m [Le bois, dans les gorges de San tschang kiou (Ho Kin), à $2500 \mathrm{~m}$ d'altid.], 06. 08. 1885, Delavay 2020" (syntypes P! - barcodes P00752725, P00432461, P00432462); "Yunnan, in umbrosis prope Koutoui, supra Mo-so-yn [Lieux frais près de Koutoui, audessus de Mo so yn] 10. 08. 1889, Delavay 4097" (syntypes P! - barcodes P00432460, P00752726, P00752727).

= Carum cardiocarpum Franch., 1894, Bull. Annuel Soc. Philom. Paris (sér. 8) 6: 120.

= Cryptotaeniopsis cardiocarpa (Franch.) Dunn, 1903, J. Linn. Soc. Bot. 35: 495.

= Pternopetalum cardiocarpum (Franch.) Hand.Mazz., 1933, Symb. Sin. 7(3): 718.

Type: CHINA. "Yunnan, in silvis ad Ma-eulchan, 10. 09. 1889, Delavay 3907” (lectotype P! barcode P00432469, designated by Wang Lisong, 2008a: 110; isolectotypes K - barcode K001091317, L, NY, P! - barcode P00432468); "Yunnan, Likiang Suee-chan, alt. 4000 m, 13. 08. 1886, Delavay" (syntype P! - barcode P00432465); "Yunnan, in silvis ad San-tcha-ho supra Mo-so-yn, 04. 09. 1889, Delavay 3946" (syntype P - barcode P00432467).

= Carum sinense Franch., 1894, Bull. Annuel Soc. Philom. Paris (sér. 8) 6: 119.

= Cryptotaeniopsis sinensis (Franch.) H. Wolff, 1927, in Engler (ed.), Pflanzenreich 90(IV, 228): 177.

= Pternopetalum sinense (Franch.) Hand.-Mazz., 1933, Symb. Sin. 7(3): 719.

Type: CHINA. "Yun-nan, in silvis ad Sau-tchaho supra Mo-so-yn, 10. 08. 1889, Delavay 4098" (lectotype P! - barcode P00432450, designated here; isolectotypes NAS, P! - barcodes P00432448, P00432449, PE).

= Cryptotaeniopsis mairei Diels ex H. Wolff, 1927, in Engler (ed.), Pflanzenreich 90(IV, 228): 180.

= Pternopetalum mairei (Diels ex H. Wolff) Hand.-Mazz., 1933, Symb. Sin. 7(3): 719, "mairii”.

= Carum mairei (Diels ex H. Wolff) M. Hiroe, 1958, Umbell. Asia 1: 72, "Maireii”.

Type: CHINA. "Yünnan: südlich von Tongtchuan, auf Bergwiesen [Yunnan, south of Tong Tihouan], 2600 m ü. M., Maire 3652" (holotype UCbarcode UC386082).

= Pternopetalum longicaule Shan Renhwa, 1940, Sinensia 11(1-2): 161, tab. 2. 
Type: CHINA. "Szechuan, Sien-tian-tze, 3560 m, slope grassy place, 01. 09. 1934, C. S. Liu 1477" (holotype NAS!; isotypes NAS, PE!, SZ).

Distribution in China: NW: Gansu; Tibet: Xizang A. R.; N: Ningxia Huizu A. R., Shaanxi; SW: Sichuan, Guizhou, Yunnan. Endemic.

Pternopetalum gracillimum (H. Wolff) Hand.Mazz., 1933, Symb. Sin. 7(3): 719.

$\equiv$ Cryptotaeniopsis gracillima $\mathrm{H}$. Wolff, 1926, Acta Horti Gothob. 2: 306.

Type: CHINA. "Sze-ch'uan austr.: Ta-hsiangling, 2000-2500 m. In rupibus muscosis, 28. 05. 1922, H. Smith 2147" (lectotype UPS!, designated here; isolectotype WU).

$=$ Pternopetalum wangianum Hand.-Mazz., 1941, Oesterr. Bot. Z. 90: 123.

Type: CHINA. "W-Setschwan: Paohsing (Mupin), 30. 06. 1936, K. L. Chu 3023" (holotype WU?).

Distribution in China: NW: Gansu; Tibet: Xizang A. R.; Centr.: Hubei; SW: Sichuan, Guizhou, Yunnan. Endemic.

Pternopetalum lamellosociliare $\mathrm{Fu}$ Kuntsun, 1981, Fl. Tsinling. 1(3): 457, 401, fig. 345.

Type: CHINA. "Kansu: Wen Hsien, Tieh-lou Commune, Yang-ka-shan, Liang-ho-kou-kow, alt. 3150 m, 02. 08. 1967, C. L. Tang 1684" (holotype WUK).

Distribution in China: NW: Gansu. Endemic.

Pternopetalum latipinnulatum (Shan Renhwa) Tan Jinbo et He Xingjin, 2015, Phytotaxa 226(3): 241.

= Pternopetalum botrychioides (Dunn) Hand.Mazz var. latipinnulatum Shan Renhwa, 1940, Sinensia 11(1-2): 158.

Type: CHINA. "Szechuan: Kuan Hsien, alt. 3000-3600 ft., 06. 07. 1928, Fang Wenpei 2062" (lectotype NAS, designated here; isolectotype NAS).

Distribution in China: SW: Sichuan. Endemic.

Pternopetalum leptophyllum (Dunn) Hand.Mazz., 1933, Symb. Sin. 7(3): 719.

$\equiv$ Cryptotaeniopsis leptophylla Dunn, 1903, J. Linn. Soc. Bot. 35: 495.

Type: CHINA. "Szechuan: ravine on Mt. Omei, $4000 \mathrm{ft}$., Faber 628" (holotype K).

$=$ Cryptotaeniopsis viridis C. Norman, 1929, J. Bot. 67: 146.

$=$ Pternopetalum viride (C. Norman) Hand.Mazz., 1933, Symb. Sin. 7(3): 719.
Type: CHINA. "Western China: Mount Omi, Wilson 4931" (holotype BM; isotypes GH, K! barcode K000075730).

= Pternopetalum confusum C. Norman, 1940, J. Bot. 78: 231.

Type: CHINA. "Western China [Sichuan], shady rocks, 05. 1904, Wilson 3669" (holotype BM!).

Distribution in China: SW: Sichuan. Endemic.

Pternopetalum molle (Franch.) Hand.-Mazz., 1933, Symb. Sin. 7(3): 718.

ECarum molle Franch., 1894, Bull. Annuel Soc. Philom. Paris (sér. 8) 6: 120.

$\equiv$ Cryptotaeniopsis mollis (Franch.) Dunn, 1903, J. Linn. Soc. Bot. 35: 496.

Type: CHINA. "Yun-nan, in silvis ad Sau-tchaho, supra Mo-so-yn, 10. 08. 1889, Delavay 4095" (lectotype P! - barcode P00432455, designated here; isolectotypes K! - barcodes K001091318, K001091319, NY - barcode NY004-05779, P! barcodes P00432456, P00432457).

= Pimpinella radiata $\mathrm{W}$. W. Sm., 1911 Rec. Bot. Surv. India 4(5): 266.

= Acronema radiatum (W. W. Sm.) H. Wolff, 1927, in Engler (ed.), Pflanzenreich 90(IV, 228): 324.

= Pternopetalum radiatum (W. W. Sm.) P. K. Mukh., 1971, Indian Forester 97(1): 55.

Type: INDIA. "Sikkim, Yeumtong (Lachung), 11000 ft., 23. 08. 1892, Gammie 992" (lectotype CAL, designated by Mukherjee, 1971: 55; isolectotypes E!, K).

= Pternopetalum longicaule Shan Renhwa var. humile Shan Renhwa et Pu Fating, 1978, Acta Phytotax. Sin. 16(3): 76.

$=$ Pternopetalum brevium (Shan Renhwa et $\mathrm{Pu}$ Fating) Fu Kuntsun, 1981, Fl. Tsinling. 1(3): 400, fig. 344.

Type: CHINA. "Shaansi: Meixian, Mt. Taibai, Dou-Mu-Gong, east gorge, 28. 09. 1937, Liou, Tsoong 1310" (holotype PE!).

$=$ Pternopetalum trifoliatum Shan Renhwa et $\mathrm{Pu}$ Fating, 1989, Acta Phytotax. Sin. 27(1): 64, fig. 3.

Type: CHINA. "Sichuan: Ma-erh-k'ang, Mt. Tihu, $3500 \mathrm{~m}$, on moss under Abies forest, 01. 09. 1984, Zhou, Pu Fating 211" (holotype CDBI!).

Distribution in China: NW: Gansu; Tibet: Xizang A. R., Qinghai; N: Ningxia Huizu A. R., Shaanxi; Centr. Hubei; SW: Sichuan, Yunnan.

Distribution outside China: Bhutan, India.

Pternopetalum monophyllum Tan Jinbo et $\mathrm{He}$ Xingjin, 2014, Ann. Bot. Fennici 51(6): 415, figs. $1-5$. 
Type: CHINA. "Sichuan Province, Tianquan County, Erlang Mountain, Maliuping, on grassy slopes in forests, $2100 \mathrm{~m}$ a.s.1., 14. 04. 2013, Tan, Ma T2013041401" (holotype SZ; isotype SZ).

Distribution in China: SW: Sichuan. Endemic.

Pternopetalum nudicaule (H. Boissieu) Hand.Mazz., 1933, Symb. Sin. 7(3): 718.

$\equiv$ Cryptotaeniopsis nudicaulis H. Boissieu, 1906, Bull. Soc. Bot. France 53: 427; et 1906, Bull. Acad. Geogr. Bot. 16: 184.

Type: CHINA. "Yunnan-sen distr., près d'une grotte Kouy-Tcheou, 05. 03. 1904, Cavalerie 2113" (lectotype P! - barcode P00432471, designated here; isolectotypes E!, P! - barcode P00432472).

= Pternopetalum nudicaule $(\mathrm{H}$. Boissieu) Hand.Mazz. var. esetosum Hand.-Mazz., 1933, Symb. Sin. 7(3): 718 .

Type: CHINA. "China: Hunan: Im schattigen wpt. Laubhochwalde des Yun-schan bei Wukang, Tonschiefer, 900-1200 m, 09. 08. 1917, HandelMazzetti 11224" (lectotype WU! - barcode WU0025939, designated here; isolectotypes E!, WU - barcode WU0025938).

Distribution in China: Centr.: Hunan, Jiangxi; SW: Sichuan, Guizhou, Yunnan; S: Guangxi Zhuang A. R., Guangdong.

Distribution outside China: Vietnam.

Pternopetalum rosthornii (Diels) Hand.-Mazz., 1933, Symb. Sin. 7(3): 719.

三 Pimpinella rosthornii Diels, 1901, Bot. Jahrb. Syst. 29 (3-4) (Fl. Centr. China): 495.

$\equiv$ Cryptotaeniopsis rosthornii (Diels) H. Wolff, 1926, Acta Horti Gothob. 2: 306.

Type: CHINA. "Sze-ch'uen, Kin shan [Nanchuan Xian, Mt. JinFo], in ditsh. 23. 07. 1891, von Rosthorn 2" (lectotype O!, designated here; isolectotypes $\mathrm{W}$; photo LE).

Distribution in China: Centr. Hubei; SW: Sichuan Endemic.

Pternopetalum subalpinum Hand.-Mazz., 1933, Symb. Sin. 7(3): 718, tab. 11, fig. 1.

Type: CHINA. "Prov. Yunnan bor--occid.: in regione frigide temperate jugi Nisselaka inter fluvios Landsang-djiang (Mekong) et Lu-djiang (Salween), $28^{\circ}$ in Abietetis lateris orient. 3700-4100 m, 18. 06. 1916, Handel-Mazzetti 8975" (lectotype WU! barcode WU0025940, designated here; isolectotypes E!, LE!, NAS, US).

Distribution in China: SW: Yunnan.

Distribution outside China: Bhutan, India.
Pternopetalum tanakae (Franch. et Sav.) Hand.Mazz., 1933, Symb. Sin. 7(3): 719.

$\equiv$ Chamaele tanakae Franch. et Sav., 1875, Enum. Pl. Jap. 1: 185.

$\equiv$ Carum tanakae Franch. et Sav., 1878, Enum.

P1. Jap. 2: 371.

$\equiv$ Pimpinella tanakae (Franch. et Sav.) Diels, 1901, Bot. Jahrb. Syst. 29 (3-4) (Fl. Centr. China): 494.

$\equiv$ Cryptotaeniopsis tanakae (Franch. et Sav.) H. Boissieu, 1902, Bull. Herb. Boissier (2 sér.) 2: 806.

Type: JAPAN. "In locis umbrosis regionis montanae: Nippon media, in silva montis Fudsiyama, 06. 1874, Savatier 3436" (holotype P!).

= Carum filicinum Franch., 1894, Bull. Annuel Soc. Philom. Paris (sér. 8) 6:121.

= Pimpinella filicina (Franch.) Diels, 1901, Bot. Jahrb. Syst. 29 (3-4) (Fl. Centr. China): 494.

= Cryptotaeniopsis filicina (Franch.) H. Boissieu, 1902, Bull. Herb. Boissier (2 sér.) 2: 806.

$=$ Pternopetalum filicinum (Franch.) Hand.Mazz., 1933, Symb. Sin. 7(3): 718.

Type: CHINA. "Central China, prov. Hupeh, Ichang, Henry 6600" (lectotype P - barcode P00432458, designated here; isolectotypes E!, LE!, $\mathrm{P}$ - barcode P00432459).

= Cryptotaeniopsis asplenioides H. Boissieu, 1902, Bull. Herb. Boissier (2 sér.) 2: 807.

$=$ Pternopetalum asplenioides $(\mathrm{H}$. Boissieu) Hand.-Mazz., 1933, Symb. Sin. 7(3): 718.

Type: CHINA. "Su-Tchien oriental, district de Tchen-Kéou-Tin, Farges" (lectotype P!, designated here; isolectotypes E, IBSC).

$=$ Pternopetalum tanakae (Franch. et Sav.) Hand.-Mazz. f. conforme Honda, 1939, Nom. Pl. Japon.: 514.

= Pternopetalum heterophyllum Hand.-Mazz., 1941, Oesterr. Bot. Z. 90: 122.

Type: CHINA. "W-Setschwan: Paohsing-hsieh (Mupin), unter Büschen, $2850 \mathrm{~m}, 07.08$. 1936, K. L. Chu 3454" (lectotype WU, designated here; isolectotypes E! - barcode E00265241, IBSC, PE, $\mathrm{SZ}$ ).

$=$ Pternopetalum tanakae (Franch. et Sav.) Hand.-Mazz. var. fulcrantum Zhang Yuhua, 1989, Bull. Bot. Res., Harbin 9(3): 59.

Type: CHINA. "Zhejiang: Suichang Xian, Mt. Jiulong, Lin 3385" (holotype ZJMA; isotype HZU).

Distribution in China: NW: Gansu; Tibet: Xizang A. R., Qinghai; N: Shanxi, Shaanxi, Hebei; Centr.: Henan, Anhui, Hubei, Hunan, Jiangxi, Zhejiang, Fujiang, SW: Sichuan, Guizhou, Yunnan.

Distribution outside China: Japan, Korea, Nepal, Bhutan?, India. 
Pternopetalum trichomanifolium (Franch.) Hand.-Mazz., 1933, Symb. Sin. 7(3): 719.

$\equiv$ Carum trichomanifolium Franch., 1895, Bull. Mus. Hist. Nat. (Paris) 1: 64.

$\equiv$ Pimpinella trichomanifolia (Franch.) Diels, 1901, Bot. Jahrb. Syst. 29 (3-4) (Fl. Centr. China): 495.

$\equiv$ Cryptotaeniopsis trichomanifolia (Franch.) H. Boissieu, 1902, Bull. Herb. Boissier (2 sér.) 2: 807.

Type: CHINA. "Yunnan, in silvis circa Longki [Les bois à Long ki], 09. 1893, Delavay [4909]" (lectotype P! - barcode P00432444, designated here; isolectotypes C! - barcode C10008535, G barcode G00367394, P00432443, P).

$=$ Cryptotaeniopsis kiangsiensis H. Wolff, 1927, in Engler (ed.), Pflanzenreich 90(IV, 228): 182.

$=$ Pternopetalum kiangsiense $(\mathrm{H}$. Wolff) Hand.Mazz., 1933, Symb. Sin. 7(3): 719.

Type: CHINA. "Südost-China: Kiang-si, Kung Shan, in feuchten Felsspalten, bei 4700` ü. M., H. H. Hu 733" (holotype K).

$=$ Cryptotaeniopsis decipiens C. Norman, 1929, J. Bot. 67: 146.

$=$ Pternopetalum decipiens $(\mathrm{C}$. Norman) $\mathrm{M}$. Hiroe, 1979, Umbelliferae World: 1013.

Type: CHINA. "Yunnan, Shweli-Salwin divide, Lat. 2540', Gongshan Xian, Forrest 18096" (lectotype BM!, designated here; isolectotypes E!, NAS).

= Pternopetalum trachycarpum Wu Zhengyi [1997, in Fl. Yunnan. 7: 535, tab. 165, fig. 1-8 (contrary to Art 37.5 of the ICBN (1994)] ex Shan Renhwa et Pan Zehui, 2007, in S.Y.Jin (ed.), Catalogue of Type Specimens (Cormophyta) in Herbaria of China, Supp. 3: 179.

Type: CHINA. "Yunnan, Jingdong, M. K. Li 3442" (holotype KUN; isotype NAS).

Distribution in China: Tibet: Xizang A. R.; Centr.: Hubei, Hunan, Jiangxi; SW: Sichuan, Guizhou, Yunnan; S: Guangxi Zhuang A.R., Guangdong. Endemic.

Pternopetalum vulgare (Dunn) Hand.-Mazz., 1933, Symb. Sin. 7(3): 719.

$\equiv$ Cryptotaeniopsis vulgaris Dunn, 1902, in Hooker's Icon. P1. 28: tab. 2737.

$\equiv$ Deringa vulgaris (Dunn) Koso-Pol., 1916, Vestnik Tiflissk. Bot. Sada 11 [1915]: 139.

= Pimpinella clarkeana Watt ex Banerji, 1951, J. Bombay Nat. Hist. Soc. 50: 88.

Type: CHINA. "Yunnan, Feng-Chen-Lin, forests, 7000', Henry 10675" (lectotype K, designated by Wang Lisong, 2008: 292; isolectotypes E!, K,
LE!, MO - barcode MO-345435); "Szechuen, Tatsienlu, 9000 to 13500 feet, Pratt 831" (syntype); "Mount Omei, 4000 to 8000 feet, Faber 60, 627, 632 (syntypes K!); "Hupeh, Chienshin, Henry 5384, 5444" (syntypes P! - barcodes P00432446, P00432447); "Patung, Henry 5406" (syntype). INDIA. "Manipur, Ching Sow, at 8000 feet, Watt 6556" (syntypes CAL, E, K!);

= Cryptotaeniopsis wolffiana Fedde ex H. Wolff, 1930, Repert. Spec. Nov. Regni Veg. 27: 327.

= Pternopetalum wolffianum (Fedde ex H. Wolff) Hand.-Mazz., 1933, Symb. Sin. 7(3): 719.

Type: CHINA. "Yunnan: Shweli-Salwin divide, $25^{\circ} 12^{\prime} \mathrm{N}, 98^{\circ} 58^{\prime}$ ' E, in shady thicket, 04. 1924, Forrest 24098" (lectotype E!, designated here; isolectotypes NY - barcode NY00405829, P! barcode P00752723, PE!).

$=$ Pternopetalum yiliangense Shan Renhwa et $\mathrm{Pu}$ Fating, 1978, Acta Phytotax. Sin. 16(3): 72, fig. 4.

Type: CHINA. "Yunnan, Yiliang Xian [NE Yunnan], alt. 1900 m, along river, 30. 05. 1932, H. T. Tsai 52081A" (lectotype IBSC, designated by Wang Lisong, 2012: 559; isolectotypes GH - barcode GH00077637, IBSC, KUN, LBG, NAS, PE!, SZ).

= Pternopetalum vulgare (Dunn) Hand.-Mazz. var. foliosum Shan Renhwa et Pu Fating, 1978, Acta Phytotax. Sin. 16(3): 69.

Type: CHINA. "Yunnan, Yongshan, under forest, 2500 m, 20. 06. 1932, H. T. Tsai 51056A" (holotype KUN; isotypes GH - barcode GH00077636, IBSC, LBG, NAS, PE, SZ).

= Pternopetalum vulgare (Dunn) Hand.-Mazz. var. strigosum Shan Renhwa et Pu Fating, 1978, Acta Phytotax. Sin. 16(3): 68.

Type: CHINA. "Sichuan: Shimian, Mt. Haizi, under forest, 18. 04. 1955, T. T. Xie 3994" (holotype $\mathrm{SZ}$; isotypes IBSC, PE).

= Pternopetalum vulgare (Dunn) Hand.-Mazz. var. acuminatum Wu Zhengyi, 1978, Acta Phytotax. Sin. 16(3): 68.

Type: CHINA. "Yunnan, Xi-shuo Co., 05. 07. 1959, Wu Quanan 7937" (holotype KUN!; isotype NAS).

= Pternopetalum molle (Franch.) Hand.-Mazz. var. dissectum Shan Renhwa et Pu Fating, 1978, Acta Phytotax. Sin. 16(3): 72.

Type: CHINA. "Yunnan: Weixi, streamside under forest, 3000-3200 m, 17. 06. 1940, K. M. Feng 4800" (holotype KUN; isotype PE!).

Distribution in China: NW: Gansu; N: Shanxi, Shaanxi, Hebei; Centr.: Hubei, Hunan, Jiangxi; SW: Sichuan, Guizhou, Yunnan; S: Guangxi Zhuang A. R. 


\section{Distribution outside China: Myanmar, Nepal, India. \\ Note: Holotype of Pimpinella clarkeana Watt ex Banerjee (Watt 6556) is among syntypes of Pternopetalum vulgare Dunn, i.e. the first name is a nomenclatural synonym of the second one.}

Pterocyclus angelicoides (DC.) Klotzsch, 1862, in Klotzsch \& Garcke (eds.), Bot. Ergebn. Reise Waldemar: 150, tab. 47.

$\equiv$ Hymenolaena angelicoides DC., 1830, Prodr. 4: 245.

$\equiv$ Pleurospermum angelicoides (DC.) Benth. ex C. B. Clarke, 1879, in Hooker f. (ed.), Fl. Brit. India 2: 703.

Type: INDIA. "Kumaon [In Nepaliae montibus altissimis, ad Kamaon et Gossain Than], Wallich [548]" (lectotype G-DC!, designated by Pimenov, Kljuykov, 2000: 522; isolectotypes BM!, CAL, E - barcode E00000207, G!, K!, K-WALLICH! barcodes K000685357, K000685358, LE!). NEPAL. "Gossain Than, Wallich [648]" (syntypes CAL, G-DC!).

Distribution in China: Tibet: Xizang A. R.; SW: Sichuan, Yunnan. India.

Distribution outside China: Bhutan, Nepal,

Pterocyclus forrestii (Diels) Pimenov et Kljuykov, 1999, Feddes Repert. 110(7-8): 485, fig. 2.

$\equiv$ Angelica forrestii Diels, 1912, Notes Roy. Bot. Gard. Edinburgh 5: 289.

Type: CHINA. "Yunnan, W. China. Shady, open situations on margins of pine forests on the eastern flank of the Lichiang Range. Lat. $27^{\circ} 20^{\prime}$ N. Alt. $11000 \mathrm{ft} ., 07$. 1901, Forrest 2580" (lectotype E! barcodes E00000165, E00000166, designated here; isolectotype $\mathrm{K}$ !).

= Pterocyclus wolffianus Fedde ex H. Wolff, 1930, Repert. Spec. Nov. Regni Veg. 27: 321.

= Pleurospermum longicarpum Shan Renhwa et Pan Zehui, 1984, Index Fl. Yunnan. 1: 923.

Type: CHINA. "Yunnan: western flank of the Shweli-Salwin divide. Lat. $25^{\circ} 20^{\prime}$ N, 10-11000', open stony pasture, 08. 1912, Forrest 8958" (lectotype E! - barcode E00000149, designated here; isolectotypes K!, NAS!).

Distribution in China: Tibet: Xizang A. R.; SW: Sichuan, Yunnan. Endemic.

Pterocyclus rivulorum (Diels) H. Wolff, in Hand.-Mazz., 1933, Symb. Sin. 7(3): 717. $\equiv$ Angelica rivulorum Diels, 1912, Notes Roy.

Bot. Gard. Edinburgh 5: 288.

$\equiv$ Pleurospermum rivulorum (Diels) Fu Kuntsun et Ho Yehchi, 1979, in Shan Renhwa \& Sheh Menglan (eds.), Fl. Reipubl. Popularis Sin. 55(1): 152, tab. 75, fig. $1-5$.

Type: CHINA. "NW Yunnan, China: Gravely boggy ground in running water on mountain meadows on the eastern flank of the Lichiang Range. Lat. $27^{\circ} 25^{\prime}$ N. Alt. 11-12000 ft., 08. 1906, Forrest 2849" (lectotype E! - barcode E00000142, designated here; isolectotype BM! - barcode BM000559387).

Distribution in China: SW: Sichuan, Yunnan. Endemic.

Pterygopleurum neurophyllum (Maxim.) Kitag., 1937, Bot. Mag. (Tokyo) 51: 655.

E Edosmia neurophylla Maxim., 1873, Bull. Acad. Imp. Sci. Saint-Petersbourg 18: 285.

$\equiv$ Carum neurophyllum (Maxim.) Franch. et Sav., 1875, Enum. Pl. Jap. 1: 180.

三Sium neurophyllum (Maxim.) H. Hara, 1954, Enum. Sperm. Jap. 3: 323.

$\equiv$ Perideridia neurophylla (Maxim.) Chuang et Constance, 1969, Univ. Calif. Publ. Bot. 55: 28, fig. 9.

Type: JAPAN. "In Kiusiu jugi centralis Kundshosan sylvis Cryptomeriae udis [Japonia, Nagasaki, Kunssho-san], 29. 09. 1863, Maximowicz" (holotype LE!).

Distribution in China: Centr.: Jiangsu, Anhui, Zhejiang.

Distribution outside China: Japan, Korea.

Rupiphila tachiroei (Franch. et Sav.) Pimenov et Lavrova, 1986, Byull. Moskovsk. Obšč. Isp. Prir. Otd. Biol. 91(2): 97.

ESeseli tachiroei Franch. et Sav., 1879, Enum. P1. Jap. 2: 373.

$\equiv$ Cnidium tachiroei (Franch. et Sav.) Makino, 1906, Bot. Mag. (Tokyo) 20: 94.

$\equiv$ Tilingia tachiroei (Franch. et Sav.) Kitag., 1937, Bot. Mag. (Tokyo) 51: 656.

$\equiv$ Ligusticum tachiroei (Franch. et Sav.) M. Hiroe et Constance, 1958, Univ. Calif. Publ. Bot. 30(1) (Umbell. Japan): 74, fig. 38.

Type: JAPAN. "In monte Asama yama, provinciae Kotske, insulae Nippon, Savatier 3790" (holotype P! - barcode P02496406).

= Ligusticum koreanum H. Wolff, 1920, Repert. Spec. Nov. Regni Veg. 17: 154.

Type: KOREA. "Hallaisan, in rupestribus, 2000 m, 10. 1907, Taquet 108" (holotype W!). 
= Cnidium affine H. Wolff, 1922, Repert. Spec. Nov. Regni Veg. Beih. 12 (Limpr. Bot. Reise China Ost-Tibet): 451, nom. illeg. (Art. 53.1), non C. A. Mey. (1849).

= Cnidium wolffii M. Hiroe, 1979, Umbelliferae World: 1325.

Type: CHINA. "Tschili: Hsiao Wu Tai Schan, felsige Abhänge oberhalb des Passes Hsien miao ling bei Li yi orl, 2200 m, Limpricht 545".

= Cnidium limprichtii H. Wolff, 1922, Repert. Spec. Nov. Regni Veg. Beih. 12 (Limpr. Bot. Reise China Ost-Tibet): 451.

$=$ Tilingia limprichtii (H. Wolff) Leute, 1970, Ann. Naturhist. Mus. Wien 74: 511.

Type: CHINA. "Schanhsi: Felsen des Tschwang ling zwischen Yi ma ling und Ling tsiu, $1600 \mathrm{~m}$, Limpricht 637" (holotype WU! - barcode WU0025962).

=Ligusticum elegans $\mathrm{H}$. Wolff, 1926, Acta Horti Gothob. 2: 312.

Type: CHINA. "Chili: Hsiao-wu-tai-shan, Yangkia-p'ing, Hsi-lin, $1900 \mathrm{~m}$, in prato alpino, 01. 09. 1921, H. Smith 1167" (lectotype UPS!, designated by M. G. Pimenov in Pimenov, Ostroumova, 2012: 226; isolectotypes GB! - barcode GB-0048814, $\mathrm{W}$ !).

= Carum bretschneideri H. Wolff, 1927, in Engler (ed.), Pflanzenreich 90(IV, 228): 369.

Type: CHINA. "Peking, 07. 1905, Schindler 74a" (syntype BM! - barcode BM000885476); "Peking, Pa hua shan, 07. 1905, Schindler 179, 500" (syntype BM!); "Berge bei Tachiaosse, Moellendorff” (syntype).

= Carum wolffianum Fedde ex H. Wolff, 1930, Repert. Spec. Nov. Regni Veg. 27: 303.

Type: CHINA. "Tchili, S. S. Chien 123".

= Carum seselifolium H. Wolff, 1930, Repert. Spec. Nov. Regni Veg. 27: 303.

Type: CHINA. "Shansi centralis: Chich-hsiehDistr., Mien-shan, in rupibus muscosis umbrosis, ca $1500 \mathrm{~m}$ s.l., 03. 10. 1924, H. Smith 7827" (lectotype UPS!, designated by M. G. Pimenov in Pimenov, Ostroumova, 2012: 226; isolectotypes BM! - barcode BM000885461, PE!, S! - barcode S-G-1189, TI!); "Shansi australis: Yuan-chu-Distr., Shui-wangping, in prato alpino harboso ca. $2400 \mathrm{~m}$ s.l., 24. 07. 1924, H. Smith 6531" (syntypes BM! - barcode BM000885462, UPS!).

= Cnidium filisectum Nakai et Kitag., 1934, Rep. First Sci. Exped. Manchoukuo, sect. IV, 1: 35, tab. 11.

$=$ Tilingia filisecta (Nakai et Kitag.) Nakai et Kitag., 1937, Bot. Mag. (Tokyo) 51: 657.

= Ligusticum filisectum (Nakai et Kitag.) M. Hiroe, 1958, Umbell. Asia 1: 105.
= Ligusticum tachiroei (Franch. et Sav.) M. Hiroe et Constance var. filisectum (Nakai et Kitag.) S. Y. He et W. T. Fan, 1988, Fl. Hebeiensis 2: 272.

Type: CHINA. "In declivitate aprica montis prope Hsing-lung, 31. 08. 1933, Nakai, Honda, Kitagawa" (holotype TI!).

Distribution in China: N: Neimenggu A. R., Ningxia Huizu A. R., Shanxi, Hebei, Shandong; NE: Heilongjiang, Jilin, Liaoning; Centr.: Henan, Anhui, Hubei, Zhejiang.

Distribution outside China: Russia, Japan, Korea.

Sanicula astrantiifolia Kretschmer ex H. Wolff, 1930, Repert. Spec. Nov. Regni Veg. 27: 308.

Type: CHINA. "Yunnan, pâtures des vallons derriere Tong-tchoua.Alt 2600 m, Maire" (lectotype P; isolectotype UC); "Yunnan, 26. 09. 1915, S. Ten 287" (syntype); "Yunnan $\mathrm{Fu}$, in silva mixta prope pagodam Tai hoa sse, $2200 \mathrm{~m}, 09$. 07. 1916, Schoch 217" (syntypes K! - barcode K000697288, US).

Distribution in China: Tibet: Xizang A. R.; Centr.: Hubei; SW: Sichuan, Yunnan. Endemic.

Sanicula caerulescens Franch., 1894, Bull. Annuel Soc. Philom. Paris, (sér. 8) 6: 109.

Type: CHINA. "Yun-nan sept., in silvis ad Tchenfong-chan, 05. 1882, Delavay 456" (lectotype P! barcode P00835131, designated here; isolectotypes K!, NY, P - barcode P00835132).

= Sanicula dielsiana H. Wolff, 1910, Repert. Spec. Nov. Regni Veg. 8: 524.

Type: CHINA. "China centralis: Provinz Sze ch'uan: Nan ch'uan, von Rosthorn 114".

= Sanicula stapfiana H. Wolff, 1913, in Engler (ed.), Pflanzenreich 61(IV, 228): 58.

Type: CHINA. "Sze ch'uan, auf dem Berge Omei, ca 2000 m hoch, Faber 887" (holotype K! barcode K000697292).

= Sanicula erythrophylla Bobr., 1950, Bot. Mater. Gerb. Bot. Inst. Komarova Akad. Nauk SSSR 13: 167.

Type: CHINA. "Prov.Szechuan, in valle fl. Ja, infra urb. Jaczchou, 03. 04. 1893, Potanin" (holotype LE!).

Distribution in China: Centr.: Hubei; SW: Sichuan, Guizhou, Yunnan.

\section{Distribution outside China: Vietnam.}

Sanicula chinensis Bunge, 1833, Mém. Acad. Imp. Sci. St.-Petersburg Divers Savans 2 (Enum. Pl. Chin. Bor.): 106.

$\equiv$ Sanicula europaea L. var. chinensis (Bunge) Diels, 1901, Bot. Jahrb. Syst. 29 (3-4) (Fl. Centr. China): 491. 
$\equiv$ Sanicula europaea L. subsp. chinensis (Bunge) Hultén, 1971, Kungl. Svenska Vetens.-akad. Handl. 13(1): 363.

Type: CHINA. "In umbrosis [montium] PaiSchan, China borealis, 1830, Bunge" (lectotype P! barcode P00602127, designated here; isolectotype LE!).

= Sanicula elata Buch.-Ham. ex D.Don var. japonica Koidz., 1930, Bot. Mag. (Tokyo) 44: 95.

$=$ Sanicula chinensis Bunge var. involucrata Nakai, 1952, Bull. Natl. Sci. Mus. 31 (Syn. Sk. Kor. Fl.): 18.

= Sanicula chinensis Bunge f. involucrata $(\mathrm{Na}-$ kai) Kitag., 1964, J. Jap. Bot. 39(9): 269.

Type: KOREA. "Prov. Zennan, monte Tjiisan, Bok-Mankei, 17. 08. 1927, Nakai" (holotype TNS!).

= Sanicula chinensis Bunge var. paupera Nakai, 1952, Bull. Natl. Sci. Mus. 31 (Syn. Sk. Kor. Fl.): 18.

= Sanicula chinensis Bunge f. paupera (Nakai) Kitag., 1964, J. Jap. Bot. 39(9): 268.

Type: KOREA. "Ins. Chejudo [Quelpaert], Mt. Hallasan, Hasawa" (holotype TNS!).

= Sanicula kurilensis Pobed., 1961, Bot. Zhurn. (Leningrad) 46(9): 1343.

Type: RUSSIA. "Ins. Kurilensis, ins. Kunashir, inter urbem Judshno-Kurilsk et pagum Gorjatchi Klutch, in silvis ad litora Pacificas, 19. 07. 1959, Pobedimova, Konovalova 848" (holotype LE!).

- Sanicula elata auct. non Buch.-Ham. ex D.

Don: Franch. \& Sav., 1875, Enum. P1. Jap. 1: 178.

- Sanicula europaea auct. non L.: Yabe, 1902,

J. Coll. Sci. Imp. Univ. Tokyo 16(2) (Rev. Umbell. Jap.): 19.

Distribution in China: NW: Gansu; N: Neimenggu A. R., Shaanxi, Shanxi, Hebei, Shandong; NE: Heilongjiang, Jilin, Liaoning; Centr.: Henan, Jiangsu, Anhui, Hubei, Hunan, Jiangxi, Zhejiang, Fujiang; SW: Sichuan, Guizhou, Yunnan; S: Guangxi Zhuang A. R. Korea.

Distribution outside China: Russia, Japan,

Sanicula elata Buch.-Ham. ex D. Don, 1825, Prodr. Fl. Nepal.: 183.

$\equiv$ Sanicula europaea L. var. elata (Buch.-Ham. ex D. Don) H. Boissieu, 1906, Bull. Soc. Bot. France 53: 421.

三 Sanicula europaea L. subsp. elata (Buch.Ham. ex D. Don) Hultén, 1971, Kungl. Svenska Vetens.-akad. Handl. 13(1): 363.

Type: NEPAL. "Narainhetty Nepalensium, 18. 11. 1802, Buchanan-Hamilton” (lectotype BM! - barcode BM000521539, designated by Shrestha in $\mathrm{BM}$; isolectotype CAL).

= Sanicula hermaphrodita Buch.-Ham. ex D. Don, 1825, Prodr. Fl. Nepal.: 183.

Type: NEPAL. "Suembu, 01. 07. 1802, Buchanan-Hamilton" (holotype BM - barcode BM000521540).

$=$ Sanicula montana Blume, 1826, Bijdr. Fl. Ned. Ind. 15: 882.

Type: INDONESIA. "[Java] in sylvis montanis locis humidis, Reinwald?"

$=$ Sanicula javanica Blume, 1826, Bijdr. Fl. Ned. Ind. 15: 882.

$=$ Sanicula europaea L. var. javanica (Blume) H. Wolff, 1913, in Engler (ed.), Pflanzenreich 61(IV, 228): 64

Type: INDONESIA. "In cacumine montis Gede [Java], Blume" (holotype L; isotype NY - barcode NY004506260).

= Sanicula elata Buch.-Ham. ex D. Don var. partita Kuntze, 1891, Revis. Gen. Pl. 1: 269.

= Sanicula europaea L. var. partita (Kuntze) M. Hiroe, 1979, Umbelliferae World: 294.

Type: INDONESIA. "Java. Gede".

= Sanicula europaea L. var. pseudoyunnanensis H. Wolff, 1929, Repert. Spec. Nov. Regni Veg. 27: 113.

Type: INDIA. "Panchmarhi, Water's meet, 23. 02. 1891, Duthie 10406".

= Eryngium moluccanum Van Steenis, 1948, Fl. Males. (ser. 1 Spermat.) 4: 595, fig. 5a.

Type: MALAYSIA. "Malaysia: Moluuucas (Central Ceram, G.Binaye, below The Gate). Eyma 2286" (holotype BO; isotypes A, K, L).

- Sanicula europaea auct. non L.: C. B. Clarke, 1879, in Hooker f. (ed.), Fl. Brit. India 2: 670.

Distribution in China: NW: Gansu; Tibet: Xizang A. R.; Centr.: Hubei; SW: Sichuan, Guizhou, Yunnan; S: Guangxi Zhuang A. R.

Distribution outside China: Philippines, Indonesia, Malaysia, Vietnam, Laos, Myanmar, Bhutan, Nepal, India, Sri-Lanka, Pakistan, Yemen, Africa.

Sanicula elongata Fu Kuntsun, 1979, in Shan Renhwa \& Sheh Menglan (eds.), Fl. Reipubl. Popularis Sin. 55(1): 297, 45, tab. 17, fig. 1-3.

Type: CHINA. "Shensi, Mei Hsien, Lao-chunLing, 17. 06. 1952. Kuo Penchao 1424" (holotype WUK).

Distribution in China: NW: Gansu; N: Shaanxi; Centr.: Henan. Endemic. 
Sanicula giraldii $\mathrm{H}$. Wolff, 1913, in Engler (ed.),

Pflanzenreich 61(IV, 228): 60.

Type: CHINA. "Nord-Schensi, südl. Lao-y-san, auf dem Berge Sciu-ian-san, am Kan-y-huo [China interior, provincia Schensi septentr. Monte Sciu-iansan, lungo il fiume Kan-y-huo, a Sud del Lao-y-san], 15. 05. 1899, Giraldi 5838" [herbarium A. Biondi] (holotype FI!).

$=$ Sanicula uralensis Kleop. ex Kamelin, Czubarov et Shmakov, 2002, Turczaninowia 5(2): 18, fig. 1, map 3 .

Type: EUROPE, RUSSIA. "Provincia Ufa, silvae montanae prope officinam metallurgicam Simsky, 20. 07. 1888, Antonov" (holotype LE!).

Distribution in China: NW: Gansu; Tibet: Xizang A. R., Qinghai; N: Ningxia Huizu A. R., Shaanxi, Shanxi, Hebei; Centr.: Henan; SW: Sichuan.

Distribution outside China: Russia, Kazakhstan, Europe.

Sanicula giraldii $\mathrm{H}$. Wolff var. ovicalycina Shan Renhwa et Liou Shoulu, 1979, in Shan Renhwa \& Sheh Menglan (eds.), Fl. Reipubl. Popularis Sin. 55(1): 297, 63, tab. 27, fig. 5-6.

$\equiv$ Sanicula subgiraldii Shan Renhwa, 1943, Sinensia 14: 112.

Type: CHINA. "Szechwan, Nanchuan Hsien, 01. 05. 1930, Chang 277" (holotype NAS).

Distribution in China: SW: Sichuan. Endemic.

Sanicula hacquetioides Franch., 1894, Bull. Annuel Soc. Philom. Paris (sér. 8) 6: 110.

Type: CHINA. "Yun-nan, in valle supra Fangyang-tchang, alt. $3300 \mathrm{~m}, 07.06 .1888$, Delavay 3470" (syntypes GH, K! - barcode K000697279, NY, P - barcode P03226509); "Yun-nan, in lapidosis calcareis septentrionalem versus, prope collum Yentze-hay, alt. 3300 m, 25. 05. 1887, Delavay 2261" (lectotype P! - barcode P03226504, designated here; isolectotype K! - barcode K000697278).

Distribution in China: NW: Gansu; Tibet: Xizang A. R., Qinghai; SW: Sichuan, Guizhou, Yunnan. Endemic.

Sanicula lamelligera Hance, 1878, J. Bot., n.s. 7(16): 11.

Type: CHINA. "Secus fluvium, North River, prov. Cantonensis, 03. 1877, Bullock (Hance) 19942" (lectotype K!, designated here; isolectotype BM - barcode BM000944682).

= Sanicula satsumana Maxim., 1886, Bull. Acad. Imp. Sci. Saint-Petersbourg 31: 47.
Type: JAPAN. "Japonia [Kiusiu], prov. Satsuma, in ipsa urbe Kago-sima, sub arboribus, 10. 1883, Tashiro" (holotype LE!).

= Sanicula yunnanensis Franch., 1894, Bull. Annuel Soc. Philom. Paris (sér. 8) 6: 108.

Type: CHINA. "Yun-nan sept., in pratis secus flumen Yang-tze-kiang, ad Kiou-pa-tan, 29. 03. 1882, Delavay 453" (lectotype P! - barcode P03226688, designated here; isolectotypes A barcode A00589886, NY - barcode NY00406259, $\mathrm{P}$ - barcode P03226687).

= Sanicula ichangensis H. Wolff, 1913, in Engler (ed.), Pflanzenreich 61(IV, 228): 54.

Type: CHINA. "Prov. Hupeh: Ichang, Henry 3525a" (lectotype K!, designated here; isolectotypes GH, P).

= Sanicula orthacantha S.Moore var. longispina H. Wolff, 1913, in Engler (ed.), Pflanzenreich 61(IV, 228): 55.

Type: CHINA. "West Hupeh, Ichang. 16. 03. 1900, Wilson 156" (lectotype K!, designated here; isolectotype E!); "Tien-Tai, in einen Hohe von 3000 m, Faber" (syntype); "Che-kiang: Ning-po, Schindler 456a" (syntype B-destr.).

= Sanicula wakayamensis Masam., 1941, Trans. Nat. Hist. Soc. Formosa 31: 340.

$=$ Sanicula lamelligera Hance var. wakayamensis (Masam.) Murata, 1962, Acta Phytotax. Geobot. 20: 195.

Type: JAPAN. "Honsyu, Kamegawa, Kaisogun, Wakayamken, Syozi".

Distribution in China: Centr.: Anhui, Hubei, Jiangxi, Zhejiang, Fujiang; SW: Sichuan, Guizhou, Yunnan; S: Taiwan, Guangxi Zhuang A. R., Guangdong.

Distribution outside China: Japan.

Sanicula nanchuanensis Shan Renhwa, 1943, Sinensia 14: 111.

Type: CHINA. "Szechwan, King Fu Shan, Nanchuan Hsien, Fong-feng-szu, alt. 2000 m, in shady place, 09. 06. 1935, K. L. Chu 1212" (holotype NAS).

Distribution in China: SW: Sichuan. Endemic.

Sanicula orthacantha S.Moore, 1875, J. Bot. 13: 227.

Type: CHINA. "Kiukiang [300 miles from the east coast and 250 above Nankin, the banks of the Yang-tse-kiang], 1873, Shearer" (lectotype K! barcode K000697283, designated here; isolectotype BM); “China, 04. 1900, Wilson 151-a” (epitype P! barcode P03226647). 
= Sanicula henryi H. Wolff, 1913, in Engler (ed.), Pflanzenreich 61(IV, 228): 55.

Type: CHINA. "Yunnan, Mengtze, 8000', Henry 10500" (lectotype K! - barcode K000697284, designated here; isolectotypes B, E! - barcode E00000049, FI?, MO, NY - barcode NY00406257, US - barcode US00126973).

= Sanicula costata H. Wolff, 1913, in Engler (ed.), Pflanzenreich 61(IV, 228): 56.

Type: CHINA. "Nord-Schensi, auf dem Berge Hua-tzo-pin, Giraldi 519".

Distribution in China: NW: Gansu; N: Shaanxi, Hebei; Centr.: Henan, Jiangsu, Anhui, Hubei, Hunan, Jiangxi, Zhejiang, Fujiang; SW: Sichuan, Guizhou, Yunnan; S: Guangxi Zhuang A. R., Guangdong.

Distribution outside China: Vietnam.

Sanicula orthacantha S.Moore var. brevispina H. Boissieu, 1906, Bull. Soc. Bot. France 53: 421.

Type: CHINA. "Chine occidentale, Mt. Omei, Wilson 7104" (holotype P! - barcode P03226637).

Distribution in China: N: Shaanxi; SW: Sichuan. Endemic.

Sanicula oviformis Liu Xintian et Liu Zhenyu, 1991, Acta Phytotax. Sin. 29(5): 471, fig. 2.

Type: CHINA. "Sichuan: Nanchuan, alt. 650 m, in moist grass, 28. 06. 1983, Sheh Menglan 83646" (holotype NAS!).

Distribution in China: SW: Sichuan. Endemic.

Sanicula pengshuiensis Sheh Menglan et Liu Zhenyu, 1991, Acta Phytotax. Sin. 29(5): 469.

Type: CHINA. "Sichuan: Pengshui, suburb., alt. $500 \mathrm{~m}$, in moist place by streams, 11. 04. 1979, Tan Sixian 0106" (holotype SMI; isotype NAS!).

Distribution in China: SW: Sichuan. Endemic.

Sanicula petagnioides Hayata, 1908, J. Coll. Sci. Imp. Univ. Tokyo 25(19): 103, tab. 12.

Type: CHINA. "Formosa, Mt. Morrison, ad 7500 ped. alt., 15. 10. 1906, Kawakami et Mori, 1988, 2026" (syntype TAIF); "Formosa, Tozan, in montibus Morison, 11. 1906, Nakahara" (syntypes TI!, TAIF).

Distribution in China: S: Taiwan. Endemic.

Sanicula rubriflora F. Schmidt ex Maxim., 1859, Mém. Acad. Imp. Sci. Saint-Petersbourg (sér. 7) 9 (Prim. Fl. Amur.): 123.

Type: RUSSIA. "Am unter Amur, unterhalb der Dondon-Mündung, in Laubwäldern am Flusse des Geong-Rückens: Zjanka, an feuchten Stellen im
Hochwalde, sehr zerstreut. 24. 05. 1855. Maximowicz" (lectotype LE!, designated by M. G. Pimenov (1987: 211); isolectotypes P - barcodes P03226624, P03226631); "Dole, gleicher Standort, nicht häufig. 21. 05. 1855. Maximowicz" (syntype LE!); "Dshare, im Laubwalde und am Waldrande, stellenweise, nicht selten. 18. 07. 1855. Maximowicz" (syntype LE!).

Distribution in China: N: Neimenggu A. R.; NE: Heilongjiang, Jilin, Liaoning.

Distribution outside China: Russia, Japan, Korea.

Sanicula rugulosa Diels, 1900, Bot. Jahrb. Syst. 29 (3-4) (Fl. Centr. China): 491.

Type: CHINA. "Setchuen. Nan ch'uan: Mai t'an ts 'ao, Berghäng, 14. 09. 1891, von Rosthorn 898" (holotype O!).

Distribution in China: Tibet: Xizang A. R.; SW: Sichuan. Endemic.

Sanicula serrata H. Wolff, 1913, in Engler (ed.), Pflanzenreich 61(IV, 228): 56.

Type: CHINA. "West-Hupeh, Patang, 04. 1900, Wilson 156A" (lectotype K!, designated here; isolectotypes E, HBG - barcode HBG510909, NY barcode NY00406258, US).

= Sanicula potaninii Bobrov, 1950, Bot. Mater. Gerb. Bot. Inst. Komarova Akad. Nauk SSSR 13: 168.

Type: CHINA. "Prov. Szechuan, urbs Dadsian-lu (= Tantsien-lou, tibetice Tarsando), in ea vicinitate, 18. 05. 1893, Potanin" (lectotype LE!, designated by Vinogradova (2010: 53); isolectotype PE!).

Distribution in China: NW: Gansu; Tibet: Xizang A. R., Qinghai; N: Shaanxi; Centr.: Hubei; SW: Sichuan, Yunnan.

Sanicula serrata H. Wolff var. uncinata Shan Renhwa et Constance, 1951, Univ. Calif. Publ. Bot. 25(1): 25 .

Type: CHINA. "Sikang, Chato Valley, neighbourhood of Tatsien-lu [Ta-chien-lu], 1923, Cunningham 37" (holotype E!).

Distribution in China: SW: Sichuan. Endemic.

Sanicula tienmuensis Shan Renhwa et Constance, 1951, Univ. Calif. Publ. Bot. 25(1): 23, fig. 40, map 41.

Type: CHINA. "Chekiang: West Tien-mu-shan, Wu-lin-ting, 30. 04. 1931, West Lake Museum 67" (holotype NAS). 
Distribution in China: Centr.: Zhejiang; SW: Sichuan. Endemic.

Sanicula tienmuensis Shan Renhwa et Constance var. pauciflora Shan Renhwa et Pu Fating, 1989, Acta Phytotax. Sin. 27(1): 66, fig. 5.

Type: CHINA. "Sichuan: Ludung, alt. 2200 $\mathrm{m}$, under forests or by streams, 01. 05. 1984, Li Yongjiang 115" (holotype CDBI!).

Distribution in China: SW: Sichuan. Endemic.

Sanicula tuberculata Maxim., 1867, Bull. Acad. Imp. Sci. Saint-Petersbourg 11: 431.

Type: KOREA. "In aestuario Chu-san peninsulae Koreanae, 1859, Wilford 906" (lectotype LE!, designated by Grubov in LE (Kwak et al., 2013: 139); isolectotypes $\mathrm{GH}$ - barcode GH00589885, K!barcode K000697277).

Distribution in China: NE: Heilongjiang.

Distribution outside China: Japan, Korea.

Saposhnikovia divaricata (Turcz.) Schischk., 1951, in Schischkin (ed.), Fl. URSS 17: 54, 359.

$\equiv$ Stenocoelium divaricatum Turcz. [ex Besser, 1834, Flora 17(1, Beibl.): 13, nom. inval. (Art. 39.1)], 1844, Bull. Soc. Imp. Naturalistes Moscou 17: 734 .

$\equiv$ Siler divaricatum (Turcz.) Benth. et Hook. f. ex Franch. et Sav., 1873, Enum. Pl. Jap. 1: 186.

$\equiv$ Laser divaricatum (Turcz.) Thell., 1925, Le Monde des Plantes N 153: 4.

$\equiv$ Ledebouriella divaricata (Turcz.) M. Hiroe, 1958, Umbell. Asia 1: 92.

Type: RUSSIA. "In campis transbaicalensibus et dahuricis copiosae provenit [In campis ad Strelcam], 06. 1829, Turczaninow" (lectotype LE!, designated by Vinogradova (2005: 966); isolectotypes C barcode C10005760, K!, KW, MW!); "In campis transbaicalensibus, 1830, Turczaninow" (syntypes K - barcode K000681169, LE!, M! - barcode M0173017, P! - barcode P00757858); "In pratis siccis prope Selenginsk, 1829, Turczaninow" (syntype LE!).

$=$ Trinia dahurica Turcz. ex Besser, 1834, Flora 17(1), Beibl.: 14, nom. inval. (Art. 39.1).

= Siler divaricatum (Turcz.) Benth. et Hook. f. ex Franch. et Sav. f. latifolium H. Wolff, 1926, Acta Horti Gothob. 2: 328.

Type: CHINA. "Chili: Hsiao-wu-tai-shan, Yang-kiap'ing, Pei-ko, 08. 1921, H. Smith 1003" (holotype GB!).

- Ledebouriella seseloides auct. non H. Wolff: Noda, 1971, Fl. NE province (Manchuria) of China: 858.
- Saposhnikovia seseloides Kitag., 1979, Neolin. Fl. Manshur.: 488, non Rumia seseloides Hoffm.

Distribution in China: NW: Xinjiang Weiwuer A. R., Gansu; N: Neimenggu A. R., Ningxia Huizu A. R., Shaanxi, Shanxi, Hebei, Shandong; NE: Heilongjiang, Jilin, Liaoning; Centr.: Henan, Hubei.

Distribution outside China: Russia, Korea, Mongolia.

Scandix stellata Banks et Sol., 1794, in Russel (ed.), Nat. Hist. Aleppo (ed. 2) 2: 249.

三Scandicium stellatum (Banks et Sol.) Thell., 1919, Repert. Spec. Nov. Regni Veg. 16: 16.

Type: SYRIA. "Aleppo, sine loco exacto, Russel” (holotype BM).

= Scandix pinnatifida Vent., 1800, Descr. P1. Nouv.: tab. 14.

$=$ Chaerophyllum pinnatifidum (Vent.) Poir., 1804, in Lamarck et al. (eds.), Encycl. 5: 144.

= Birostula pinnatifida (Vent.) Raf., 1840, Good Book: 59.

Type: IRAN. "Sur la route d'Amadan à Tehran coll. Bruguière \& Olivier., introduite chez Cels (Paris), Ventenat" (holotype G - barcode G00341470).

$=$ Scandix pinnatifida Vent. var. songarica Schrenk, 1841, Enum. P1. Nov. 1: 62.

Type: KAZAKHSTAN. "Semina lecta versus montes Labassy. 14. 06. 1840, Schrenk" (holotype LE?).

$=$ Scandix pinnatifida Vent. var. hirsuta K. Koch, 1842, Linnaea 16: 363.

Type: GEORGIA. "In Georgia prope Tiflis et in Armenia, K. Koch".

= Scandix russeliana Griseb., 1843, Spic. Fl. Rumel. 1: 369.

Type: IRAQ. "In Mesopotamia, Donietti" (holotype GOET - barcode GOET011410).

= Scandix hispidula Bertol., 1842, Nov. Comm. Acad. Bologn. 5: 427.

Type: IRAQ. "Ex vallo castri Sedjim Kala. Chesney?".

$=$ Scandix pinnatifida Vent. var. decipiens Bornm., 1898, Verh. Zool.- Bot. Ges. Wien 48: 593.

Type: LEBANON. "Libanon: In der Alpenregion des Sannin bei 2300 m, am Rand der Schneefelder zwischen Felsen zusammen mit Lamium Ehrenbergii Boiss., 21. 05. 1910, Bornmüller 11823" (holotype JE!).

= Scandix pinnatifida Vent. f. subulata Bornm., 1898,Verh. Zool.- Bot. Ges. Wien 48: 593.

Type: IRAN. "Kermanshah: Kuh Gahwarren. 20. 05. 1909, Strauss" (holotype JE). 
= Scandix fedtschenkoana Koso-Pol., 1916, Izv. Imp. S.-Peterburgsk. Bot. Sada 16: 227.

Type: TURKMENISTAN. "In regione transcaspica, in montibus Bolchie Balchany, prope Djebel, 20. 04. 1912, Andrjusczenko" (holotype LE!).

= Scandix manjurkiana Tamamsch., 1932, Bot. Arch. 34: 524.

Type: ARMENIA. "In Armenien, Nahe von Erivan zwischen Djervesch und Woachaberd, 13. 06. 1931, Tamamschjan" (holotype ERE?).

= Scandicium stellatum (Banks et Sol.) Thell. var. eriophorum Bornm., 1945, in Koie (ed.), Danish Sci. Invest. Iran 4: 14.

Type: IRAN. "Kechwar, 700-900 m, Koie 888".

Distribution in China: NW: Xinjiang Weiwuer A. R.

Distribution outside China: Russia, India, Pakistan, Kazakhstan, Kirghizia, Tajikistan, Uzbekistan, Turkmenistan, Afghanistan, Iran, Azerbaijan, Georgia, Armenia, Turkey, Iraq, Saudi Arabia, Yemen, Lebanon, Syria, Israel, Jordan, Egypt, Cyprus, Greece (E Aegean Ils.). Europe, Africa.

Schrenkia vaginata (Ledeb.) Fisch. et C. A. Mey., 1841, in Schrenk, Enum. Pl. Nov. 1: 64.

三Cachrys vaginata Ledeb., 1829, Fl. Altaic. 1: 366.

$\equiv$ Anidrum vaginatum (Ledeb.) Koso-Pol., 1916, Bull. Soc. Imp. Naturalistes Moscou, s.n. 29: 146.

$\equiv$ Bifora vaginata (Ledeb.) Koso-Pol., 1916, Bull. Soc. Imp. Naturalistes Moscou, s.n. 29: 147, in adnot.

Type: KAZAKHSTAN. "[Altai] In montibus Dolenkara deserti Soongoro-Kirghisici, 1826, Meyer 229" (lectotype LE!, designated here; isolectotypes $\mathrm{C}$ - barcode $\mathrm{C} 10008551, \mathrm{H}$ - barcode H1393929, HAL).

= Schrenkia vaginata (Ledeb.) Fisch. et C. A. Mey. var. transitoria Lipsky, 1904, Trudy Imp. S.Peterburgsk. Bot. Sada 23: 160.

= Anidrum transitorium (Lipsky) Koso-Pol., 1920, Izv. Glavn. Bot. Sada RSFSR 36(1) (Fl. As. Ross. 15): 85.

$=$ Schrenkia transitoria (Lipsky) Kamelin, 1973, Bot. Zhurn. (Leningrad) 58(5): 630, quoad nomen, cum basyon. auct. Koso-Pol.

Type: TAJIKISTAN. "Jagnob, Margif, 18. 07. 1882, Komarov" (holotype LE!).

= Schrenkia mogoltavica O.E.Politova, 1978, Byull. Moskovsk. Obšč. Isp. Prir. Otd. Biol. 83(4): 140.
Type: TAJIKISTAN. "Turkestan, prov. Samarkand, montes Mogol-Tau prope pagum Chilkovo, 04. 06. 1916, Androssov 1790" (holotype LE!).

Distribution in China: NW: Xinjiang Weiwuer A. R.

Distribution outside China: Kazakhstan, Kirghizia, Tajikistan, Uzbekistan.

Schulzia albiflora (Kar. et Kir.) Popov, 1940, Fl. Alma-Atinsk. Zapovedn.: 35.

三Chamaesciadium albiflorum Kar. et Kir., 1842, Bull. Soc. Imp. Naturalistes Moscou 15: 360.

三Schulzia crinita (Pall.) Spreng. subsp. albiflora (Kar. et Kir.) Pimenov, 1983, in Vvedensky (ed.), Opred. Rast. Sred. Azii 7: 263.

Type: KAZAKHSTAN. "In herbidis subalpinis et alpinis Alatau ad fl. Sarchan, 07. 1841, Karelin, Kirilov 1514" (lectotype MW!, designated by Gubanov (1993); isolectotypes BM!, BR - barcode BR0000005419586, G - barcodes G00367262, G00367277, H - barcode H1395759, K! - barcodes K000695716, K000695717, LE!, M!, NY, P!, TK, W!, WU!).

= Chamaesciadium albiflorum Kar. et Kir. var. subacaulis Trautv., 1866, Bull. Soc. Imp. Naturalistes Moscou 39(2): 317.

Type: KAZAKHSTAN. "In montibus Alatau, 07. 1841, Schrenk" (lectotype LE!, designated here; isolectotype BM!); "In pylis Kokatau, 06. 07. 1841, Schrenk" (syntype LE); "In alpis ad fl. Baskan, 29. 07. 1841, Schrenk" (syntype LE); "In montibus Dschill-Karagai, 20. 06. 1841, Schrenk" (syntype LE).

= Chamaesciadium albiflorum Kar. et Kir. var. caulescens Trautv., 1866, Bull. Soc. Imp. Naturalistes Moscou 39(2): 317.

Type: KAZAKHSTAN. "In excursione versus montes Saratau, 07. 1841, Schrenk" (holotype LE).

= Ligusticum baltistanicum H. Wolff, 1930, Repert. Spec. Nov. Regni Veg. 27: 310.

Type: INDIA. "Baltistan, Karpuchu Valley, 13-14000 ft., 10. 07. 1892, Duthie 11956" (lectotype $\mathrm{K}$ !, designated here; isolectotypes BM, E!, L); "Baltistan, Nar nullah, above Dras, 12-13000 ft., 03. 07. 1892, Duthie 11794" (syntype K!).

- Schulzia dissecta auct. non C. Norman: Nasir, 1972, in Nasir \& Ali (ed.), Fl. W Pakistan 20: 113, fig. 33, A-C.

Distribution in China: NW: Xinjiang Weiwuer A. R.

Distribution outside China: India, Pakistan, Kazakhstan, Kirghizia, Tajikistan, Uzbekistan. 
Schulzia crinita (Pall.) Spreng., 1813, Neue Schr. Naturf. Ges. Halle 2, 1 (Pl. Umbell. Prodr.): 30.

$\equiv$ Sison crinitum Pall., 1779, Acta Acad. Sci. Imp. Petrop. 2: 250, tab. 7.

$\equiv$ Sium crinitum (Pall.) Poir., 1811, in Lamarck et al. (eds.), Encycl. Suppl. 1(2): 622.

$\equiv$ Athamanta crinita (Pall.) Ledeb., 1829, Fl. Altaic. 1: 326 .

$\equiv$ Carum crinitum (Pall.) Koso-Pol., 1916, Bull. Soc. Imp. Naturalistes Moscou, s.n. 29: 198.

Type: RUSSIA. "In nivalibus montium Altaicorum ingis, viginti circiter stadia a fortalitia et rivo Tigerek versus occidentalem [In alpibus altaicis], 1791, Pallas" (lectotype K!, designated by M. G. Pimenov in Pimenov, Ostroumova, 2012: 210; isolectotypes B-Willd!, BM!, HAL!, P!).

Distribution in China: NW: Xinjiang Weiwuer A. R.

Distribution outside China: Russia, Mongolia, Kazakhstan.

Schulzia dissecta (C. B. Clarke) C. Norman, 1938, J. Bot. 76: 231.

$\equiv$ Trachydium dissectum C. B. Clarke, 1879, in Hooker f. (ed.), Fl. Brit. India 2: 672.

$\equiv$ Aulacospermum dissectum (C. B. Clarke) U. Dhar, Kachroo et Naqshi, 1995, in Kachroo (ed.), Central Asia \& Kashmir Himalaya: 107, nom. inval. (Art. 41.5).

Type: INDIA. "Sikkim, Tungu, alt. 1300014000 ft., 10. 1848, Hooker Cortia 3" (lectotype K! - barcode K000695933, designated by M. F. Watson (1998: 378); isolectotypes GH, K barcodes K000695932, K000695934, P - barcode P03224724).

Distribution in China: Tibet: Xizang A. R.

Distribution outside China: Bhutan, Nepal, India.

Schulzia prostrata Pimenov et Kljuykov, 1990, Bot. Zhurn. (Leningrad) 75(1): 94, fig. 5, 6.

Type: KIRGHIZIA. "Kirghizia, regio Naryn, distr, At-baschy, Tian-Schan Centralis, in systemate fluminis Aksai, vall. fl. Terek, in glareosis inundatis, $\mathrm{h}=3500$ m, 08. 08. 1987, Pimenov, Kljuykov 790" (holotype LE!; isotypes FRU!, MW!).

- Trachydium tianschanicum auct. non Korovin: Pu Fating, 1979, in Shan Renhwa \& Sheh Menglan (eds.), Fl. Reipubl. Popularis Sin. 55(1): 203, tab. 107, fig. 1-8.

Distribution in China: NW: Xinjiang Weiwuer A. R.
Distribution outside China: Kirghizia.

Semenovia dasycarpa (Regel et Schmalh.) Korovin, 1981, in Czerepanov (ed.), Sosud. Rast. SSSR: 29.

$\equiv$ Pastinaca dasycarpa Regel et Schmalh., 1877, Trudy Imp. S.-Peterburgsk. Bot. Sada 5(2): 598.

$\equiv$ Zosima dasycarpa (Regel et Schmalh.) Korovin, 1924, Bot. Mater. Gerb. Glavn. Bot. Sada RSFSR 5, 6: 82.

$\equiv$ Malabaila dasycarpa (Regel et Schmalh.) Schischk., 1951, in Schischkin (ed.), Fl. URSS 17: 262.

$\equiv$ Platytaenia dasycarpa (Regel et Schmalh.) Korovin, 1959, in Vvedensky (ed.), Fl. Uzbekistan. 4: 462 .

Type: KIRGHIZIA. "In Turkestaniae montibus Alexander in trajectu Karabura, 8000“ alt., Regel" (holotype LE!).

= Heracleum brignoliifolium Franch., 1883, Ann. Sci. Nat. Paris (sér. 6) 16: 297.

Type: UZBEKISTAN. "Tourpag Bell, vallée de l'Ona Oulgane, dans les monts Tschirtchik, alt. 2300 m, 23. 08. 1881, Capus 530" (lectotype P! - barcode P00753148, designated here; isolectotypes LE! (fragment), P! - barcodes P00753149, P00753150).

$=$ Platytaenia komarovii Schischk., 1951, in Schischkin (ed.), Fl. URSS 17: 357, 272.

= Semenovia komarovii (Schischk.) Manden., 1959, Trudy Tbilis. Bot. Inst. 20: 19.

= Zosima komarovii (Schischk.) M. Hiroe, 1979, Umbelliferae World: 1762.

Type: TAJIKISTAN. "Asia Media, in jugo inter fl. Muran [Churan] et Ksyl-su, in declivio occidentali montis Karimdzag [Karamanak], 27. 07. 1932, Gonczarov, Grigoriev, Nikitin 809" (lectotype LE!, designated by Kamelin (Vinogradova, 1997b: 56)).

= Platytaenia rosea Nikitina [1959, in Vvedensky (ed.), Fl. Kirgiz. SSR 8: 111, nom. inval.] 1959, in Vvedensky (ed.), Fl. Kirgiz. SSR 9: 207.

Type: KIRGHIZIA. "Fergana Mts., declivum australe, loc. Kurmaidan, 26. 06. 1956, Lebedeva" (holotype FRU!).

= Heracleum multibracteatum Rech. f., 1963, K. Danske Vid. Selsk., Biol. Skrift. 13(4) (Symb. Afghan. 5): 83.

Type: AFGHANISTAN. "NE: Nuristan: Minjan: Ptili, 2700 m, 19. 07. 1948, Edelberg 2128" (lectotype $\mathrm{W}$ !, designated here; isolectotype C! barcode C10008553).

Distribution in China: NW: Xinjiang Weiwuer A. R. 
Distribution outside China: Kazakhstan, Kirghizia, Tajikistan, Uzbekistan, Afghanistan.

Semenovia malcolmii (Hemsl. et H. Pearson) Pimenov, 2013, Nordic J. Bot. 31(6): 660, Fig. 10.

$\equiv$ Peucedanum malcolmii Hemsl. et H. Pearson, 1902, J. Linn. Soc. Bot. 35: 179.

Type: CHINA. "[Tibet] In $85^{\circ} \mathrm{E}$ and $35^{\circ} 31^{\prime}$ N [North Tibet, Asmal], 05. 09. 1896, Hedin" (lectotype K! - barcode K000685422, designated here); “[Tibet] $88^{\circ} 20^{\prime}$ E, 35 14 ' N, $16142 \mathrm{ft}$, 26.07.1896, Wellby and Malcolm" (syntype K! barcode K000685419).

= Semenovia montana Kamelin et V. M. Vinogr., 1986, Novosti Sist. Vyssh. Rast. 23: 97.

Type: CHINA. "China Austro-Occidentalis, prov. Kam, pars orientalis, vallis fl. Fu-Ban-cho, inter vicos Mardan et Ljanchokou, 07. 08. 1893, Potanin" (holotype LE!).

Distribution in China: NW: Gansu; Tibet: Xizang A. R., Qinghai; SW: Sichuan. Endemic.

Semenovia rubtzovii (Schischk.) Manden., 1959, Trudy Tbilis. Bot. Inst. 20: 23.

$\equiv$ Platytaenia rubtzovii Schischk., 1951, in Schischkin (ed.), Fl. URSS 17: 357, 273, tab. 23, fig. 5.

三 Zosima rubtzovii (Schischk.) Kamelin, 1977, Novosti Sist. Vyssh. Rast. 14: 192.

Type: KAZAKHSTAN. "Kasachstania, Montes Dzhungarski Alatau. Ditio Dzharkent, in faucibus Taldy-bulak, 18. 07. 1937, Rubtzov, Linczevski”" (holotype LE; isotype AA!).

Distribution in China: NW: Xinjiang Weiwuer A. R.

Distribution outside China: Kazakhstan.

Semenovia thomsonii (C. B. Clarke) Manden., 1959, Trudy Tbilis. Bot. Inst. 20: 23, "Thomsoni”.

$\equiv$ Heracleum thomsonii C. B. Clarke, 1879, in Hooker f. (ed.), Fl. Brit. India 2: 711.

$\equiv$ Platytaenia lasiocarpa (Boiss.) Manden. subsp. thomsonii (C. B. Clarke) Rech. f. et Riedl, 1963, K. Danske Vid. Selsk., Biol. Skrift. 13(4) (Symb. Afghan. 5): 104, figs. 68, 69.

E Semenovia lasiocarpa (Boiss.) Manden. subsp. thomsonii (C. B. Clarke) R.Alava, 1987, in Rechinger (ed.), Fl. Iranica 162: 488, tab. 450.

Type: INDIA. "Ladak and Pipi, alt. 11000$15000 \mathrm{ft}$., Thomson" (lectotype K!, designated by R. Alava (1987: 488); isolectotype CAL, P - barcode P03227832).

Distribution in China: Tibet: Xizang A. R.
Distribution outside China: India, Pakistan, Afghanistan.

Semenovia torilifolia (H. Boissieu) Pimenov, 2013, Nordic J. Bot. 31(6): 660, Fig. 9.

三Peucedanum torilifolium H. Boissieu, 1903, Bull. Herb. Boissier (2 sér.) 3(10): 852.

Type: CHINA. "Thibet orient., Ta-Tsien-Lou, principaute du Kia-La, Tongolo: forêt Takuroyu, 23. 08. 1893, Soulié 632" (holotype P! - barcode P00753009).

= Heracleum millefolium Diels, 1906, Repert. Spec. Nov. Regni Veg. 2(18): 65.

= Semenovia millefolia (Diels) V. M. Vinogr. et Kamelin, 1986, Novosti Sist. Vyssh. Rast. 23: 96.

Type: CHINA. "Tibet bor. orient.: Ri tzu, 29. 08. 1904, Filchner".

= Heracleum millefolium Diels var. longilobum C. Norman, 1933, J. Arnold Arbor. 14: 25.

= Heracleum longilobum (C. Norman) Sheh Menglan et Wang Tiehseng, 1992, in Shan Renhwa \& Sheh Menglan (eds.), Fl. Reipubl. Popularis Sin. 55(3): 209, tab. 93, fig. 6.

Type: CHINA. "Central Kansu. Lien hoa shan: among rocks between Tao Chow and Titao, alt. 11500 ft, 14-20. 07. 1925, Rock 12734" (lectotype $\mathrm{E}$ !, designated here; isolectotypes $\mathrm{GH}, \mathrm{K}$ ! - barcode K000075540, NY).

= Heracleum smithii Fedde ex H. Wolff, 1934, Repert. Spec. Nov. Regni Veg. 33: 79.

Type: CHINA. "[Sichuan] reg. bor.: Sung-pan, ca 3200 m s.1., 07. 07. 1922, H. Smith 2250" (holotype UPS!).

= Heracleum millefolium Diels var. glabrum J.Q.Liu, 1999, in Pan Jintang (ed.), Fl. Qinghaica 2: 434, 391 .

Type: CHINA. "Qinghai: Gade, ad ripas fluviorum alt. $4000 \mathrm{~m}, 09.08$. 1993, Ho, Bartholomew, Gilbert 986" (holotype HNWP).

Distribution in China: NW: Xinjiang Weiwuer A. R., Gansu; Tibet: Xizang A. R., Qinghai; SW: Sichuan, Yunnan. Endemic.

Semenovia transiliensis Regel et Herder, 1866, Bull. Soc. Imp. Naturalistes Moscou 39(2): 79.

$\equiv$ Heracleum transiliense (Regel et Herder) O. et B. Fedtsch., 1909, Consp. F1. Turkest. 3: 112.

Type: KAZAKHSTAN. "Ober Tschilik Thal Alatau transiliensis, 23. 07. 1857, Semenov" (lectotype LE!, designated by Vinogradova (2002b: 134); isolectotype US - barcode US00517444); "Alatau transiliensis, Kurmetu Pass, 1857, Semenov" (syntype LE). 
= Heracleum pimpinellifolium Rupr., 1869, Mém. Acad. Imp. Sci. Saint-Petersbourg, (ser. 7), 14(4) (Sert. Tianschan.): 49, nom. illeg. (Art. 52.1), non Spreng. (1813).

Type: KIRGHIZIA. "Schamsi-Schlucht, 09. 07. 1867, Osten-Saken" (holotype LE!).

= Heracleum angustilobatum Nikitina, 1959, in Vvedensky (ed.), Fl. Kirgiz. SSR 8: 107, nom. inval. (Art. 39.1).

Type: KIRGHIZIA. "Bassin fl. Chon-Kemin, loco Karagailun-Bulak, declivum septentrionaleoccidentalis, prata, 22. 06. 1954, Isakov" (holotype FRU!).

A. R.

Distribution in China: NW: Xinjiang Weiwuer

Distribution outside China: Kazakhstan, Kirghizia.

Seseli abolinii (Korovin) Schischk., 1950, in Schischkin (ed.), Fl. URSS 16: 505, tab. 34, fig. 5.

$\equiv$ Phlojodicarpus abolinii Korovin, 1924, Bot. Mater. Gerb. Glavn. Bot. Sada RSFSR 5(6): 74.

$\equiv$ Libanotis abolinii (Korovin) Korovin, 1963, in Pavlov (ed.), Fl. Kazakhst. 6: 351.

Type: KAZAKHSTAN. "Distr. Heptapotamica, jugum Dzhungarsky Alatau, montes Karu-Duvan, valle fl. Borochudsir, decl. saxosum, 28. 06. 1917, Abolin, Borman, Steinberg 4165" (lectotype TASH!, designated by Pimenov, 1978: 189).

$=$ Seseli songoricum Schischk., 1950, in Schischkin (ed.), Fl. URSS 16: 602, 505.

$=$ Libanotis soongorica (Schischk.) Korovin, 1963, in Pavlov (ed.), Fl. Kazakhst. 6: 350.

Type: KAZAKHSTAN. "Dzhungarski Alatau, Chaptzagai meridionalis, ad ripam affluentis contra Karaschar, alt. ca. 2400 m, 30. 08. 1879, Regel"' (holotype LE!).

= Libanotis michailoviae Korovin, 1962, Trudy Inst. Bot. Akad. Nauk Kazakh. SSR 13: 254, "michailovae".

Type: KAZAKHSTAN. "Montes DshungarskyAlatau, pars meridionalis, in angustic Suak-su, ad locos lapidosos, 22. 08. 1938, Michaylova" (holotype AA!).

$=$ Libanotis turajgyrica Bajtenov, 1968, Vestnik Akad. Nauk Kazakh. SSR 7 (279): 67.

Type: KAZAKHSTAN. "Transiliensi Alatau, montes Turajgyr, mons Serektas ad declivitate lapidosas, 29. 08. 1967, Bajtenov" (holotype AA!; isotype LE!).

$=$ Seseli langshanense Zhao Yizhi et Ma Yuchuan, 1991, Acta Sci. Nat. Univ. Intramongol. 22(3): 407.

Type: CHINA. "Nei Mongol: Bayannaoermen, Langshan, Hulougaier, in vallibus vel clivis petrosis, alt. 1800-2000 m, 07. 07. 1988, Zhao Yizhi et al. 2197" (holotype HIMC).

Distribution in China: NW: Xinjiang Weiwuer A. R.

Distribution outside China: Mongolia, Kazakhstan.

Seseli acaule (Shan Renhwa et Sheh Menglan) V. M. Vinogr., 1989, Novosti Sist. Vyssh. Rast. 26: 124, "acaulis".

$\equiv$ Libanotis acaulis Shan Renhwa et Sheh Menglan, 1983, Acta Phytotax. Sin. 21(1): 84, tab. 3, fig. 6-10.

Type: CHINA. "Xinjiang: Hejing, Bayburk, alt. 2300-2600 m, 03. 08. 1958, A. J. Li, J. N. Zhu 6378" (holotype PE; isotype KUN!).

Distribution in China: NW: Xinjiang Weiwuer A. R. Endemic.

Seseli aemulans Popov, 1940, Bot. Mat. Gerb. Bot. Inst. Komarova Akad. Nauk SSSR 8(4): 73.

Type: KAZAKHSTAN. "Alatau transiliensis, ad fl. Tscharyn, in deserto saxoso gypsaceo (hamada) prope pag. Tscharyn, inter frutices Caraganae aurantiacae, cum Sisymbrio subspinescenti, Anabasi etc., 09. 08. 1937, Popov" (lectotype LE!, designated here; isolectotype AA!).

Distribution in China: NW: Xinjiang Weiwuer A. R.

Distribution outside China: Kazakhstan.

Seseli albescens (Franch.) Pimenov et Kljuykov, 2000, Bot. Zhurn. (St. Perersburg) 85(10): 107, fig. $3, \mathrm{E}$.

$\equiv$ Pimpinella albescens Franch., 1883, Nuov. Arch. Mus. Hist. Nat., (sér. 2) 6 (Pl. David. 1): 139.

$\equiv$ Eriocycla albescens (Franch.) H. Wolff, 1927, in Engler (ed.), Pflanzenreich 90(IV, 228): 107.

Type: CHINA. "Mongolie oriental: Sartchy, dans les fontes des rochers secs dans les mount. australe de la chaîne, 08. 1866, David 2948" (holotype P! barcode P00752877).

= Seseli provostii H. Boissieu, 1903, Bull. Herb. Boissier (2 sér.) 3(11): 842.

Type: CHINA. "Sur les pentes nord près la grande muraille, Provost 48" (holotype P!).

= Eriocycla albescens (Franch.) H. Wolff var. latifolia Shan Renhwa et Yuan Changchi, 1983, Acta Phytotax. Sin. 21(1): 88.

Distribution in China: N: Neimenggu A. R., Ningxia Huizu A. R., Shanxi, Hebei; NE: Jilin, Liaoning. Endemic.

Seseli asperulum (Trautv.) Schischk., 1950, in Schischkin (ed.), Fl. URSS 16: 520, tab. 34, fig. 7. 
$\equiv$ Seseli coronatum Ledeb. var. asperulum Trautv., Trudy Imp. S.-Peterburgsk. Bot. Sada 1: 32).

Type: KAZAKHSTAN. "Songoria, 1869, Hb. Trautvetter" (holotype LE!).

Distribution in China: NW: Xinjiang Weiwuer A. R.; Tibet: Qinghai.

Distribution outside China: Kazakhstan.

Seseli buchtormense (Spreng.) W. D. J. Koch, 1824, Nova Acta Phys.-Med. Acad. Caes. Leop.Carol. Nat. Cur. 12(1): 111.

三Bubon buchtormensis Spreng., 1815, Pl. Min. Cogn. Pug. 2: 55.

三Libanotis buchtormensis (Spreng.) DC., 1829,

Col. Mém. 5 (Mém. Fam. Ombell.): 48, tab. 3, fig. $\mathrm{S}$.

$\equiv$ Hippomarathrum buchtormense (Spreng.)

Link, 1829, Handbuch 1: 349.

$\equiv$ Balinotella buchtormensis (Spreng.) Soják, 1982, Zprávy Krajsk. Vlastiv. Muz. Olomouci 215: 4.

Type: RUSSIA/KAZAKHSTAN. "In Sibiria [Ex Hort. Bot. Berol.]" (holotype B?).

$=$ Athamanta rigida Hornem., 1815, Hort. Bot. Hafn. 2: 960.

Type: not indicated.

$=$ Seseli giraldii Diels, 1900, Bot. Jahrb. Syst. 29 (3-4) (Fl. Centr. China): 497.

Type: CHINA. "N, Kuan to shan [Kuan-to-san, Shen-si septent. (Cina)]. 11. 1896. Giraldi 1335" (holotype FI!).

= Libanotis cycloloba Gilli, 1959, Repert. Spec. Nov. Regni Veg. 61(3): 196.

$=$ Seseli cyclolobum (Gilli) Pimenov et Sdobnina, 1975, Bot. Zhurn. (Leningrad) 60(8): 1120.

Type: AFGHANISTAN. "Ost-Afghanistan: Gebirge oberhalb des zwischen Gulbahar und Surobi gelegenen Nedschrab-(Nidjrau-)Tales [Kabul, Nijrao, Gebirge oberhalb des Nedschrabtales], enge Stelle in einer Schlucht, durch die ein Bächlein wasserfallartig herunterstürzt, in einer schwer erreichbaren Felsspalte, 3100 m, 13. 09. 1951, Gilli 1992" (holotype W!).

- Seseli sibiricum auct. non Garcke: Benth. ex

C. B. Clarke, 1879, in Hooker f. (ed.), Fl. Brit. India 2: 693.

- Seseli libanotis auct. non W. D. J. Koch: Nasir, 1972, in Nasir \& Ali (ed.), Fl. W Pakistan 20: 76.

Distribution in China: NW: Xinjiang Weiwuer A. R., Gansu; N: Ningxia Huizu A. R., Shaanxi, Shanxi; Centr.: Hubei; SW: Sichuan.

Distribution outside China: Russia, India, Pakistan, Mongolia, Kazakhstan, Afghanistan.
Seseli condensatum (L.) Rchb.f, 1867, in Reichenbach (ed.), Icon. Fl. Germ. Helv. 21: 37.

$\equiv$ Athamanta condensata L., 1753, Sp. pl.: 1195.

$\equiv$ Libanotis condensata (L.) Crantz, 1767, Cl. Umb. Emend.: 106.

$\equiv$ Ligusticum condensatum (L.) Spreng., 1813, Neue Schr. Naturf. Ges. Halle 2(1) (Pl. Umb. Prodr.): 40.

$\equiv$ Libanotis vulgaris DC. var. condensata (L.)

DC., 1830, Prodr. 4: 150.

$\equiv$ Peucedanum condensatum (L.) Koso-Pol., 1922, Sched. Herb. Fl. Ross. 8: 115.

$\equiv$ Pachypleurum condensatum (L.) Korovin, 1963, in Pavlov (ed.), Fl. Kazakhst. 6: 310.

Type: RUSSIA. "[Described on plants, grown at Uppsala from seeds, collected by P. G. Demidov in "Siberia", probably near Solikamsk], 345.5" (lectotype LINN - barcode LINN-HL345-5, designated by Jarvis, Knees (1988) in Taxon 37(2): 474).

=Ligusticum vaginatum Spreng., 1815, Pl. Min. Cogn. Pug. 2: 57.

$=$ Libanotis fastigiata Rupr., 1845, Beitr. Pflanzenk. Russ. Reiches 2: 36, in textu.

$=$ Libanotis arctica Rupr., 1845, Beitr. Pflanzenk. Russ. Reiches 2: 36.

= Seseli arcticum (Rupr.) Calest., 1905, Webbia 1: 203.

= Libanotis condensata (L.) Crantz subsp. arctica (Rupr.) V.G.Sergienko, 1980, Novosti Sist. Vyssh. Rast. 17: 245.

Type: RUSSIA. "[N Ural] Epromont, Mikulkin" (holotype LE?).

= Carum takenakai Kitag., 1951, J. Jap. Bot. 26(6): 166.

Type: CHINA. "Prov. Hepei: in monte Hsiaowu-tai-shan, 08. 1938, Takenaka 88" (holotype TI!).

Distribution in China: NW: Xinjiang Weiwuer A. R.; N: Neimenggu A. R., Shanxi, Hebei.

Distribution outside China: Russia, Mongolia, Kazakhstan, Europe.

Seseli coronatum Ledeb., 1829, Fl. Altaic. 1: 336.

Type: KAZAKHSTAN. "[Altai] In deserto soongoro-kirghizico, 1826, Meyer 264" (lectotype LE!, designated here; isolectotypes C - barcode C10008562?, P! - barcode P02496371).

- Seseli tschuiliense auct. non Korovin: Yang Chunyu, 1985, Clav. Pl. Xinjiang. 3: 403.

- Hyalolaena tschuiliensis auct. non Pimenov et Kljuykov: V. M. Vinogr., 1994, in Rast. Tsentral'noi Azii 10: 57. 
A. R.

Distribution in China: NW: Xinjiang Weiwuer

Distribution outside China: Kazakhstan.

Seseli delavayi Franch., 1894, Bull. Annuel Soc. Philom. Paris (sér. 8) 6: 130.

Type: CHINA. "Yun-nan, in collibus calcareis montis Lo-ho-chan, supra Che-tong, prope Tapintze, alt. $1500 \mathrm{~m}$ [Les côteaux calcaires du Lo ko chan au dessus du The tong près du Ta pin tze], 04. 09. 1885, Delavay 2024" (lectotype P! - barcode P04197843, designated here; isolectotypes K! barcode K000697465, P! - barcodes P02284799, P02284800); "China Yun-nan, in collibus incultis ad Chouang-che-teou, prope Tapin-tze, 23. 08. 1888, Delavay 3510" (syntypes C! - barcode C10008563, K! - barcode K000697464, P! - barcode P02284798, P04197844).

Distribution in China: SW: Yunnan. Endemic.

Seseli eriocarpum (Schrenk) B. Fedtsch., 1915, Rastit. Turkest.: 617.

$\equiv$ Libanotis eriocarpa Schrenk, 1843, Bull. Phys.-Math. Acad. Petersb. 2: 195.

Type: KAZAKHSTAN. "Songorei, in collibus aridis mont. Ulutau, 07. 1842, Schrenk 622" (syntypes LE!, MANCH!); "Songarei, grasreiche Steppen am Kara-Kingir, 10. 06. 1842, Schrenk 621" (lectotype LE!, designated by Vinogradova (2001: 53)).

Distribution in China: NW: Xinjiang Weiwuer A. R.

Distribution outside China: Mongolia, Kazakhstan.

Seseli eriocephalum (Pall. ex Spreng.) Schischk., 1950, in Schischkin (ed.), Fl. URSS 16: 518, tab. 34, fig. 3.

$\equiv$ Bubon eriocephalus Pall. ex Spreng., 1824, Syst. Veg. (ed. 16) 1: 900.

$\equiv$ Seseli lessingianum Turcz. ex Kar. et Kir. var. eriocephalum (Pall.) O. et B. Fedtsch., 1909., Consp. Fl. Turkest. 3: 92.

Type: KAZAKHSTAN. "Lacum Inder, Pallas?" (holotype LINN).

= Seseli lessingianum Turcz. ex Kar. et Kir., 1841, Bull. Soc. Imp. Naturalistes Moscou 14: 429.

$=$ Lomatopodium lessingianum (Turcz. ex Kar. et Kir.) Fisch. et C. A. Mey., 1845, Bull. Phys.-Math. Acad. Petersb. 3: 305.

Type: KAZAKHSTAN. "In deserto Kirghisico orientaliore [Deserto Kirgisorum], Lessing" (lectotype LE!, designated by Vinogradova (2002b: 135); isolectotype MW!).
$=$ Lomatopodium platyphyllum Schrenk, 1845, Bull. Phys.-Math. Acad. Petersb. 3: 306.

$=$ Seseli platyphyllum (Schrenk) O. et B. Fedtsch., 1909, Consp. Fl. Turkest. 3: 94.

Type: KAZAKHSTAN. "Songaria, Balchasch [In litore fluvii Chatynssu], 25. 07. 1843, Schrenk 347" (lectotype LE!, designated here; isolectotypes BP!, G! - barcode G00367368, JE - barcode JE00019224, K! - barcode K000697453, MANCH!, MW!).

= Lomatopodium karelinianum Turcz. ex Stschegl., 1854, Bull. Soc. Imp. Naturalistes Moscou 27(1): 167.

Type: KAZAKHSTAN. "In salsis deserti Soongaro-Kirghisici inter Kurtschum et lacum Noor-Saissan, 1840, Karelin, Kirilov" (lectotype LE!, designated by Gubanov et al. (1998: 64); isolectotype MW!).

Distribution in China: NW: Xinjiang Weiwuer A. R.

Distribution outside China: Russia, Kazakhstan, Uzbekistan.

Seseli glabratum Willd. ex Spreng., 1820, in Roemer \& Schultes (eds.), Syst. Veg. (ed. 15 bis) 6: 406.

Type: RUSSIA. "In Sibiria, Pallas W5945" (holotype B-Willd!).

= Seseli tenuifolium Ledeb., 1829, Fl. Altaic. 1: 333; 1829, Icon. Pl. Altaic. 1: 23, tab. 97.

Type: KAZAKHSTAN. "In apricis siccis sterilibus prope fortalitium Ustkamenogorsk [locis apricis pr. Ustkamenogorsk et simelibus locis in deserto occidental. trans. fl. Irtysch], Meyer 260" (lectotype LE!, designated by Vinogradova (2002b: 136); isolectotypes B?, C!, HAL?); "In deserto Kirghisorum inter Ustkamenogorsk et Ablaikit, Meyer" (syntypes LE!, P! - barcodes P02496622, P02496623).

Distribution in China: NW: Xinjiang Weiwuer A. R.

Distribution outside China: Russia, Mongolia, Kazakhstan, Uzbekistan.

Seseli grubovii V. M. Vinogr. et Sanchir, 1985, Bot. Zhurn. (Leningrad) 70(7): 965, fig. 1.

$=$ Libanotis grubovii (V. M. Vinogr. et Sanchir) Sheh Menglan et M. F. Watson, 2004, Acta Phytotax. Sin. 42(6): 563.

Type: CHINA. "Dzhungaria, transitus inter Tian-Schan et Bogdo-Ola, prope pagum Saepu ad lacum Saepu, in deserto submontano, 05. 09. 1929, Popov 423" (holotype LE!). 
Distribution in China: NW: Xinjiang Weiwuer A. R.

Distribution outside China: Mongolia.

Seseli incanum (Steph. ex Willd.) B. Fedtsch., 1915, Rastit. Turkest.: 617.

$\equiv$ Athamanta incana Steph. ex Willd., 1798, Sp. P1. (ed. 4) 1 (2): 1402.

$\equiv$ Libanotis incana (Steph. ex Willd.) O. et B. Fedtsch., 1909, Consp. Fl. Turkest. 3: 94.

Type: KAZAKHSTAN. "Habitat in Sibiria [Altay, near Ust-Kamenogorsk], Stephan" (holotype B-Willd!).

=Libanotis patriniana DC., Prodr. 4: 150. 1830 .

Type: KAZAKHSTAN. "In collo schistoso arido ad ripam flum. Irtisch, in Sibiria altaica ad arcem Oust-Kamenogorsk, Patrin".

= Libanotis graveolens Ledeb., 1829, Fl. Altaic. 1: 340 .

= Seseli graveolens (Ledeb.) Ledeb., 1829, F1. Altai. 1: 340; 1830, Icon. P1. Altaic. 3: 6, tab. 104, nom. illeg. (Art. 53.1), non Scop. (1771).

Type: KAZAKHSTAN. "In rupestribus rarius prope fortalitium Ustkamenogorsk, nec non frequens trans.fl. Irtysch, versus ruinas Ablakit, 1826, Meyer 179" (lectotype LE!, designated by Vinogradova (2002b: 135); isolectotypes B!, HAL?). A. R.

Distribution in China: NW: Xinjiang Weiwuer

Distribution outside China: Kazakhstan.

Seseli intramongolicum Ma Yuchuan, 1979, F1. Intramongolica 4: 207, 171, tab. 79.

Type: CHINA. "Inner Mongolia (Nei Mongol), Baotou Shi, Guyang Xian, Babiang, Chasitai Shan, alt. $1500 \mathrm{~m}$, in rocky crevice on the summit, 28. 08. 1978, Ma Yuchuan 78-80" (holotype HIMC; isotype LE!).

Distribution in China: NW: Gansu; N: Neimenggu A. R., Ningxia Huizu A.R. Endemic.

Seseli jinanense (Xu Lingchuan et Xu Mingde) Pimenov, 1999, Feddes Repert. 110(7-8): 487.

$\equiv$ Libanotis jinanensis $\mathrm{Xu}$ Lingchuan et $\mathrm{Xu}$ Mingde, 1989, Bull. Bot. Res., Harbin 9(1): 37.

Type: CHINA. "Shandong, Jinan, Licheng, alt. 500-600 m, 27. 10. 1986, L. C. Xu 85101” (syntype NAS); "Shandong, Jinan, Licheng, 08. 1987, M. D. Xu 87101" (syntype NAS).

Distribution in China: N: Shandong. Endemic.

Seseli junatovii V. M. Vinogr., 1985, Novosti Sist. Vyssh. Rast. 22: 198, fig. 1.
Type: CHINA. "Dzungaria, jugum Dzhair, in angustiis ad septemtrionem a trajectu Dzhair, 4-5 $\mathrm{km}$ ad meridiem a statione militario Yamatu, in via ex Aktam ad Czuguszak ducente, zona stepposa, in fissuris ad declive lapidosum, 04. 08. 1957, Junatov et al. 1147" (holotype LE!).

Distribution in China: NW: Xinjiang Weiwuer A. R. Endemic.

Seseli lancifolium (Fu Kuntsun) Pimenov, 1999, Feddes Repert. 110(7-8): 487.

$\equiv$ Libanotis lancifolia Fu Kuntsun, 1975, Acta Phytotax. Sin. 13(2): 59, fig. 9-13.

Type: CHINA. "Shaanxi, Huaying Xian, alt. 580 m, 18. 10. 1973, Fu Kuntsun 17194” (holotype NWBI).

Distribution in China: NW: Gansu; N: Shaanxi, Shanxi, Hebei, Shandong; Centr.: Henan. Endemic.

Seseli lanzhouense (Fu Kuntsun) V. M. Vinogr., 1985, Novosti Sist. Vyssh. Rast. 22: 200.

$\equiv$ Libanotis lanzhouensis Fu Kuntsun, 1983, Acta Phytotax. Sin. 21(1): 84, tab. 3, fig. 1-5.

Type: CHINA. "Gansu: Lanzhou, Wuquan Shan, Y. L. Liu 134” (holotype NWTCI).

Distribution in China: NW: Gansu; Tibet: Qinghai. Endemic.

Seseli laticalycinum (Shan Renhwa et Sheh Menglan) Pimenov, 1999, Feddes Repert. 110(7-8): 487.

$\equiv$ Libanotis laticalycina Shan Renhwa et Sheh Menglan, 1983, Acta Phytotax. Sin. 21(1): 82.

Type: CHINA. "Shanxi: Ruicheng, alt. 1600 m, S. Y. Bao, S. J. Yan 484" (holotype PE).

Distribution in China: N: Shanxi. Endemic.

Seseli libanotis (L.) W. D. J. Koch, 1824, Nova Acta Phys.-Med. Acad. Caes. Leop.-Carol. Nat. Cur. 12(1): 111, fig. 49, 50.

$\equiv$ Athamanta libanotis L., 1753, Sp. pl.: 244.

$\equiv$ Libanotis montana Crantz, 1767, Stirp. Austr. 3: 117 .

三Ammi daucifolium Scop., 1771, Fl. Carniol. (ed. 2) 1: 207, nom. illeg. (Art. 52.1)

$\equiv$ Athamanta collina Salisb., 1796, Prodr. Stirp.

Chap. Allerton: 168, nom. illeg. (Art. 52.1).

$\equiv$ Bubon libanotis (L.) Dumort., 1827, Fl. Belg.: 79.

三Libanotis vulgaris DC., 1830, Prodr. 4: 150.

$\equiv$ Athamanta riviniiana Host ex Steud., 1840, Nomencl. Bot. (ed. 2) 1: 166, nom. illeg. (Art. 52.1).

ESeseli vulgare Bubani, F1. Pyren. 2: 376. 1900, nom. illeg. (Art. 52.1). 
$\equiv$ Selinum libanotis (L.) E. H. L. Krause, 1904, in Sturm (ed.), Deutschl. Fl. (ed. 2), 12: 102, tab. 18.

$\equiv$ Balinotella libanotis (L.) Soják, 1982, Zprávy

Krajsk. Vlastiv. Muz. Olomouci 215: 3.

Type: EUROPE. "In Sueciae, Germaniae pratis siccis apricis, 345.1" (lectotype LINN, designated by Pardo (1981: 168)).

= Libanotis daucoides Scop., 1771, Fl. Carniol. (ed. 2) 1: 192.

= Athamanta daucoides J.F.Gmelin, 1791, Syst. Nat. (ed. 13) 2(1): 477.

= Athamanta daucifolia (Scop.) Host, 1827, Fl. Austriac. I: 362.

= Libanotis daucifolia Rchb., 1832, Fl. Germ. Excurs.: 468.

Type: AUSTRIA. "[Carniolica] In alpibus Vochinensibus".

$=$ Athamanta pubescens Retz., 1783, Obs. Bot. 3: 28 .

= Ligusticum athamantoides Spreng., 1813, Neue Schr. Naturf. Ges. Halle 2(1) (Pl. Umb. Prodr.): 40.

= Seseli athamantoides Ledeb., 1829, Fl. Altaic. 1: 342 .

$=$ Libanotis sibirica C. A. Mey., 1831, Verz. Pfl. Casp. Meer.: 124.

Type: RUSSIA. "[Altai] In campestribus ubique, Ledebour" (syntype LE?); "ad fl. Irtysch, 14. 08. 1826, Meyer 219" (lectotype LE!, designated by V. M. Vinogradova (2005: 965)).

$=$ Seseli athamantoides Ledeb. var. acaule Ledeb., 1829, Fl. Altaic. 1: 342.

Type: KAZAKHSTAN. "Ad rivulum Tschaganka prope mont. Tschingistan deserti soongoro-kirghisici locis fertilibus subhumidis, 14 . 08. 1826, Meyer 219" (lectotype LE, designated by V. M. Vinogradova, 2005).

= Libanotis intermedia Rupr., 1845, Beitr. Pflanzenk. Russ. Reiches 4: 53.

= Seseli libanotis (L.) W. D. J. Koch subsp. intermedium (Rupr.) P. W. Ball, 1968, Feddes Repert. 79(1-2): 64.

$=$ Seseli intermedium (Rupr.) Vodopyanova, 1979, in Malyshev (ed.), Fl. Tsentral'noi Sibiri 2: 681, fig. 23.2, map 965.

Type: EUROPE, RUSSIA. "In umbrosis ad pedem montis Duderhof, 1842, Zobel" (holotype LE!).

= Seseli sibiricum Garcke, 1849, Fl. N. Mitt.Deutschland (ed. 1): 139.

$=$ Seseli libanotis (L.) W. D. J. Koch var. longilobum Boiss., 1872, Fl. Orient. 2: 967.

Type: TURKEY. "In subhumidis Armeniae Turcicae ad pagum Gestemert prope Gumgum prov. Musch alt. 5200', 16. 08. 1859, Kotschy 311" (holotype G-BOIS).

- Libanotis sibirica auct. non C. A. Mey.: Krylov, 1935, Fl. Zapadnoi Sibiri 8: 2069.

- Libanotis transcaucasica auct. non Schischk.: Achundov, 1955: 464, p.p.

- Seseli transcaucasicum auct. non Pimenov et Sdobnina: Mandenova, 1984: 231.

Distribution in China: NW: Xinjiang Weiwuer A. R.

Distribution outside China: Russia, Kazakhstan, Iran, Azerbaijan, Georgia, Armenia, Turkey, Europe, Africa, N. America.

Seseli mucronatum (Schrenk) Pimenov et Sdobnina, 1973, Byull. Moskovsk. Obšč. Isp. Prir. Otd. Biol. 78(4): 139.

$\equiv$ Neogaya mucronata Schrenk, 1843, Enum. Pl. Nov. 2: 40; 1842, Bull. Phys.-Math. Acad. Petersb. 1: 80 .

$\equiv$ Pachypleurum mucronatum (Schrenk) Schischk., 1950, in Schischkin (ed.), Fl. URSS 16: 581, tab. 34, 37.

E Ligusticum mucronatum (Schrenk) Leute, 1970, Ann. Naturhist. Mus. Wien 74: 473.

Type: KAZAKHSTAN. "In montibus Alatau [Songarei, Alatau], 18. 07. 1841, Schrenk 850" (lectotype LE!, designated here; isolectotype MANCH!).

$=$ Ligusticum thomsonii C. B. Clarke, 1879, in Hooker f. (ed.), Fl. Brit. India 2: 698.

Type: INDIA. "Tibet occid., Reg. temp. Zanskar, 11000-13000 ped., 29. 06. 1848, Thomson" (syntypes BM, G! - barcode G00367247, K barcode K000695854); “Tibet occ. Reg. temp. alt. 11-13000 ped., Nubra, 01.08.1848, Thomson, 2 Oreocome" (lectotype W!, designated by Leute (1970:490); isolectotypes E! - barcode E00265213, G, K! - barcodes K000695851, K000695852, MH!, NY - barcode NY00406027, P! - barcode P03221131).

= Pleurospermum longicaule H. Wolff, 1929, Repert. Spec. Nov. Regni Veg. 27: 117.

Type: CHINA. 'Kansu: upper Kap Ching K'ou, near Old Taochow, 3800-4300 m s.1., 28-31. 08. 1923, Ching 878" (holotype US!).

= Neogaya urbis-malorum Popov, 1935, Index Sem. Hort. Bot. Alma-Ata 2: 14, nom. inval.

Type: KAZAKHSTAN. "Mts Alatau transiliensis, ad fontes rivuli Kazashka. Mt. Kumbel, ca. 2500 m, declivia herbosa, 08. 08. 1933, Popov, Linczevski" (holotype LE!).

=Libanotis subsimplex Popov, 1957, Index Sem. Hort. Bot. Alma-Ata 2: 13. 
Type: KAZAKHSTAN. "[Alatau transiliensis] Mts. Kungei Alatau, ad fontes fluminis Tschilik, declivia pratensia in valle rivuli Tjulku-bulak, 17. 08. 1934, Popov" (neotype AA!, designated here).

$=$ Libanotis dolichostyla Schischk., 1950, in Schischkin (ed.), Fl. URSS 16: 600, 480.

$=$ Pachypleurum dolichostylum (Schischk.) Korovin ex Kamelin, 1971, in Ovczinnikov (ed.), Fl. \& Rastit. Uchel. Reki Varsob: 194.

= Seseli dolichostylum (Schischk.) M. Hiroe, 1979, Umbelliferae World: 1126, "dolichostyla".

Type: KIRGHIZIA. "Asia Media, Montes TjanSchan. Ad ripam fl. Karakol occident., 22. 07. 1908, Roshevitz" (holotype LE!).

Distribution in China: NW: Xinjiang Weiwuer A. R., Gansu; Tibet: Xizang A. R., Qinghai; N: Shaanxi; SW: Sichuan, Yunnan.

Distribution outside China: India, Pakistan, Mongolia, Kazakhstan, Kirghizia, Tajikistan, Uzbekistan, Afghanistan.

Seseli nudum (Lindl.) Pimenov et Kljuykov, 2000, Bot. Zhurn. (St. Perersburg) 85(10): 105, fig. $1, \mathrm{~A}, \mathrm{~B}$.

$\equiv$ Eriocycla nuda Lindl., 1835, in Royle (ed.), Ill. bot. Himal. Mts.: 232, tab. 51, fig. 2.

$\equiv$ Pituranthos nudus (Lindl.) Benth. ex C. B. Clarke, 1879, in Hooker f. (ed.), Fl. Brit. India 2: 680.

Type: INDIA. "Himalaya bor.-occid., Rarung in Kunawer, alt. 10,000-11,000 ft, 1846, Royle" (lectotype $\mathrm{K}$ !, designated here; isolectotypes BM, LE!).

= Scaphespermum trilobum Edgew., 1845, Proc. Linn. Soc. London 1: 253, "Schaphespermum".

$=$ Seseli trilobum (Edgew.) C. B. Clarke, 1879, in Hooker f. (ed.), Fl. Brit. India 2: 694.

Type: INDIA. "Himala, in glareosis, alt. ped. 10000-11000, prope Niti, Edgeworth 172" (holotype K!).

= Seseli nortonii Fedde ex H. Wolff, 1930, Repert. Spec. Nov. Regni Veg. 27: 329.

Type: CHINA. "Tibet, Mt. Everest, Plunglem valley, scree, 11500', 16. 06. 1922, Norton 108" (holotype K!).

Distribution in China: Tibet: Xizang A. R.

Distribution outside China: Nepal, India, Pakistan.

Seseli nudum (Lindl.) Pimenov et Kljuykov var. purpurascens (Shan Renhwa et Yuan Changchi) Pimenov et Kljuykov, comb. nov. $\equiv$ Eriocycla nuda Lindl. var. purpurascens Shan Renhwa et Yuan Changchi, 1980, Acta Phytotax. Sin. 18(3): 376.

Type: CHINA. "Xizang: Zayii Xian (Tsawarung), alt. 2900 m, 08. 1965, C. W. Wang 65302" (holotype $\mathrm{PE})$.

Distribution in China: Tibet: Xizang A. R. Endemic.

Seseli pelliotii (H. Boissieu) Pimenov et Kljuykov, 2000, Bot. Zhurn. (St. Petersburg) 85(10): 105, fig. 2, E.

EPituranthos pelliotii H. Boissieu, 1910, Bull. Mus. Hist. Nat. (Paris) 16: 164.

$\equiv$ Eriocycla pelliotii (H. Boissieu) H. Wolff, 1927, in Engler (ed.), Pflanzenreich 90(IV, 228): 106.

Type: CHINA. "Turkestan chinois, prov. de KouTchar, vallée de Gair, à 1500 m, 03. 08. 1907, Pelliot, Vaillant 436" (holotype P! - barcode P00752857).

$=$ Platytaenia depauperata Schischk., 1951, in Schischkin (ed.), Fl. URSS 17: 356, 270.

= Semenovia depauperata (Schischk.) Manden., 1959, Trudy Tbilis. Bot. Inst. 20: 22.

= Zosima depauperata (Schischk.) M. Hiroe, 1979, Umbelliferae World: 1761.

= Seseli depauperatum (Schischk.) V. M. Vinogr., 1981, in Czerepanov (ed.), Sosud. Rast. SSSR: 30.

Type: KIRGHIZIA. "Kirghizia, Montes TianSchan, ad ripas Kiczik-Usungusch, $4 \mathrm{~km}$ supra ostium, in clivis herbosis lapidosisque, 01. 09. 1939, Jakovleva 235" (holotype LE!).

Distribution in China: NW: Xinjiang Weiwuer A. R.

Distribution outside China: Kirghizia.

Seseli purpureovaginatum Shan Renhwa et Sheh Menglan, 1980, Acta Phytotax. Sin. 18(3): 377.

Type: CHINA. "Xizang: Biru Xian, alt. 3800 $\mathrm{m}$, in locis fruticibus ad clivos apricos montium et in fissuris rupium secum fluvios, 09. 09. 1976, Qinghai-Xizang Exped. 11346" (holotype PE).

Distribution in China: Tibet: Xizang A. R. Endemic.

Seseli sandbergiae Fedde ex H. Wolff, 1930, Repert. Spec. Nov. Regni Veg. 27: 309.

Type: CHINA. "Shansi, Shui-io. Sandberg 75" (holotype L).

= Seseli schansiense Fedde ex H. Wolff, 1930, Repert. Spec. Nov. Regni Veg. 28: 109. 
Type: CHINA. 'Shansi: Shueiyn, 3000', 15. 07. 1920, Th. Ghg. 117".

Distribution in China: N: Shanxi. Endemic.

Seseli schrenkianum (C. A. Mey. ex Schischk.) Pimenov et Sdobnina, 1975, Bot. Zhurn. (Leningrad) 60(8): 1119.

$\equiv$ Libanotis schrenkiana C. A. Mey. ex Schischk., 1950, in Schischkin (ed.), Fl. URSS 16: 601, 478.

$\equiv$ Balinotella schrenkiana (C. A. Mey. ex Schischk.) Soják, 1982, Zprávy Krajsk. Vlastiv. Muz. Olomouci 215: 4.

Type: KAZAKHSTAN [described on the basis of plants, grown in St. Petersburg Botanical Garden from seeds, collected by A. Schrenk in East Kazakhstan] (holotype LE!).

- Libanotis sibirica auct. non C. A. Mey.: O. \& B. Fedtsch., 1909, Consp. Fl. Turkest. 3: 95.

Distribution in China: NW: Xinjiang Weiwuer A. R.

Distribution outside China: Kazakhstan, Kirghizia, Tajikistan, Uzbekistan.

Seseli seseloides (Turcz.) M. Hiroe, 1958, Umbell. Asia 1: 135.

$\equiv$ Libanotis seseloides Turcz., 1844, Bull. Soc. Imp. Naturalistes Moscou 17: 725.

$\equiv$ Ligusticum seseloides Fisch. et C. A. Mey. ex Turcz., 1844, Bull. Soc. Imp. Naturalistes Moscou 17: 725 .

$\equiv$ Balinotella seseloides (Turcz.) Soják, 1982, Zprávy Krajsk. Vlastiv. Muz. Olomouci 215: 4.

Type: RUSSIA. "In campis Dahuriae ad limites Chinenses [In campis Dahuriae Nerczinensis], 1831, Turczaninow" (lectotype LE!, designated by Vinogradova, 2005: 963; isolectotypes LE!, M! barcode M0172882, P! - barcodes P03221094, P03221095, P03221097).

$=$ Libanotis montana Crantz var. riviniana Ledeb., 1844, Fl. Ross. 2: 279, non Libanotis riviniana Scop. (1772).

=Libanotis amurensis Schischk., 1950, Bot. Mater. Gerb. Bot. Inst. Komarova Akad. Nauk SSSR 13: 160 .

= Seseli riviniianum (Ledeb.) M. Hiroe, 1979, Umbelliferae World: 1126.

Type: RUSSIA. "Orient Extremus, in systemate fl. Amur, in pratis ad ripam sinistram fl. Anjuj (Dondon), contra ostium fl. Monema, 02. 09. 1926, Neymark 523" (holotype LE!).

= Seseli laserpitiifolium Palib., 1906, Bull. Herb. Boissier (sér. 2) 6: 19.

= Libanotis laserpitiifolia (Palib.) Fu Kuntsun, 1981, Fl. Tsinling. 1(3): 411.
Type: CHINA. "Planta e seminibus, in China boreali sylvis Weichang dictae, a J. W. Palibin Octobr. Mens. anno 1899 lectis in horto Petropolitano enata [dans la Chine septentrionale, dans les forêts de Weichang. à $15 \mathrm{~km}$ au SW de la station missionaire à Tung-kia-yang-tze" (holotype LE!).

Distribution in China: NW: Gansu; N: Neimenggu A. R., Ningxia Huizu A. R., Hebei, Shandong; NE: Heilongjiang, Jilin, Liaoning; Centr.: Henan, Jiangsu, Hubei.

Distribution outside China: Russia, Korea, Mongolia.

Seseli sessiliflorum Schrenk, 1845, Bull. Phys.Math. Acad. Petersb. 3: 307.

Type: KAZAKHSTAN. "In montibus Chantau, locis arenosis, 24. 06. 1843, Schrenk" (lectotype LE!, designated here; isolectotype P! - barcode P02496637; isolectotype P!).

= Seseli squarrosum Schischk., 1950, Bot. Mater. Gerb. Bot. Inst. Komarova Akad. Nauk SSSR 13: 162 .

Type: KAZAKHSTAN. "Kasachstania, distr. Karsakpai, ad marginem occidentalem deserti Bedpak-dala, in loco Kamys-bulak, 14. 08. 1929, Smirnov 622" (holotype LE!).

Distribution in China: NW: Xinjiang Weiwuer A. R.

Distribution outside China: Kazakhstan, Kirghizia.

Seseli simplicifolium (Wu Zhengyi) Pimenov et Kljuykov, 1999, Feddes Repert. 110(7-8): 488.

$=$ Seseli mairei $\mathrm{H}$. Wolff var. simplicifolium $\mathrm{Wu}$ Zhengyi, 1983, Acta Phytotax. Sin. 21(1): 88.

Type: CHINA. "Sichuan: Hui dong, alt. 2300 m, 04. 10. 1964, Yue Jinsan 64180" (holotype NAS).

Distribution in China: SW: Sichuan, Yunnan. Endemic.

Seseli spodotrichomum (Fu Kuntsun) Pimenov, 1999, Feddes Repert. 110(7-8): 487.

$\equiv$ Libanotis spodotrichoma Fu Kuntsun, 1975, Acta Phytotax. Sin. 13(2): 58, fig. 1-8.

Type: CHINA. "Shaanxi, Hu Xian, Laoyudonghe, alt. 1100 m, 19. 09. 1962, Fu Kuntsun 14804" (holotype NWBI; isotype KUN!).

Distribution in China: N: Shaanxi. Endemic.

Seseli strictum Ledeb., 1829, Fl. Altaic. 1: 338.

$\equiv$ Athamanta stricta (Ledeb.) Ledeb. ex Steud. (ed.), 1840, Nomencl. Bot. (ed. 2) 1: 166.

Type: RUSSIA. "In pratensibus ad radices montium altaicorum frequens v.gr. prope Sogra, 
Loktewsk, Smejow alibique (L., M.) [Loktewsk], Ledebour, Meyer 257" (lectotype LE!, designated by Vinogradova (2005: 965); isolectotypes C! barcode C10008566, P! - barcode P02496631); "Altai, Sogra, 24. 08. 1826, Ledebour 1151" (syntype LE!).

= Pseudammi ehrenbergii H. Wolff, 1920, Repert. Spec. Nov. Regni Veg. 17: 173.

= Ammi ehrenbergii (H. Wolff) M. Hiroe, 1979, Umbelliferae World: 1025.

Type: RUSSIA. "Sibiria occidentalis: inter Omsk et Petropavlowsk, 1829, Ehrenberg" (syntype); "Altai: loco et tempore non indicates, Ehrenberg" (syntype).

Distribution in China: NW: Xinjiang Weiwuer A. R.

Distribution outside China: Russia, Kazakhstan, Europe.

Seseli togasii (M. Hiroe) Pimenov et Kljuykov, 1999, Feddes Repert. 110(7-8): 488.

三Deverra togasii M. Hiroe, 1979, Umbelliferae World: 504, fig. 149.

Type: CHINA. "Asia, Manchuria. Prov. Chilin (Suiyan): Saratsi, alt. 1000 m, rupestrine, Togasi" (holotype KYO).

Distribution in China: NE: Jilin. Endemic.

Seseli vaillantii H. Boissieu, 1910, Bull. Mus. Natl. Hist. Nat. (Paris) 16: 165.

Type: CHINA. "Turkestan chinois, prov. de Kutchar, Zamutek-Tagh, mine d'Ammone, à $2000 \mathrm{~m}$ [Asie Centrale, Zamutek tagh (Ammone)], 30. 07. 1907, Pelliot, Vaillant 382" (holotype P! - barcodes P02496432, P02496433).

= Seseli fedtschenkoanum Regel et Schmalh. var. iliense Regel et Schmalh., 1881, Izv. Obšč. Ljubit. Estestv. 34(2) (Descr. Pl. Nov. Rar. Fedtsch.): 31.

$=$ Seseli iliense (Regel et Schmalh.) Lipsky ex B. Fedtsch., 1915, Rastit. Turkest.: 616.

= Libanotis iliensis (Regel et Schmalh.) Korovin, 1963, in Pavlov (ed.), Fl. Kazakhst. 6: 345.

Type: KAZAKHSTAN. "In valle fl. Ili in angustis Koibin, 1876, Regel" (lectotype LE!, designated by Pimenov (1978: 190); isolectotypes K! - barcode K000697451, P - barcode P02496318).

= Seseli altissimum Popov, 1940, Bot. Mat. Gerb. Bot. Inst. Komarova Akad. Nauk SSSR 8(4): 72.

Type: KAZAKHSTAN. "Tianschan orientalis, regio iliensis, in angustiis fluminis Tscharyn loco Kurtogai dicto, in rupibus porphyricis, 12. 08. 1937, Popov, Goloskokov" (holotype AA!; isotype LE!).

Distribution in China: NW: Xinjiang Weiwuer A. R.
Distribution outside China: Kazakhstan.

Seseli valentinae Popov, 1940, Bot. Mat. Gerb. Bot. Inst. Komarova Akad. Nauk SSSR 8(4): 73.

Type: KAZAKHSTAN. "Tianschan orientalis, jugum Kungei Alatau ad flumen Tschilik ad rivulum Saty, in collibus siccis rubris gypsaceis, 22. 08. 1937, Michailova" (lectotype LE!, designated by Vinogradova (2002b: 136); isolectotype AA!).

$=$ Lomatopodium lessingianum (Turcz.) Fisch. et C. A. Mey. var. tenuifolium Regel et Herder, 1866, Bull. Soc. Imp. Naturalistes Moscou 39(3): 75.

Type: KAZAKHSTAN. "Keskelen-Thale im Alatau transiliensis, Semenov" (holotype LE!).

Distribution in China: NW: Xinjiang Weiwuer A. R.

Distribution outside China: Kazakhstan, Kirghizia.

Seseli veitchii (H. Boissieu) Pimenov, comb. nov.

三Peucedanum veitchii $\mathrm{H}$. Boissieu, 1906, Bull. Soc. Bot. France 53: 436.

Type: CHINA. "Western China, the yellow cliffs, 4000-7500 ft., 08. 1903, Wilson 3705" (lectotype P! - barcode P00753003, designated here; isolectotypes BM - barcode BM000885415).

= Seseli incisodentatum Fu Kuntsun, 1981, in Fl. Tsinling. 1(3): 459, 412, fig. 353.

Type: CHINA. "Kansu, Wen Hsien, prope urbem, alt. $920 \mathrm{~m}, 10.10 .1958$, C. P. Wei 2813" (holotype WUK).

$=$ Seseli squarrulosum Shan Renhwa et Sheh Menglan, 1983, Acta Phytotax. Sin. 21(1): 86, tab. 4, fig. 1-6.

Type: CHINA. "Qinghai: Tongren, alt. 3100 m, 16. 06. 1972, Kuo Penchao 10099" (holotype NWBI; isotype NAS!).

Distribution in China: NW: Gansu; Tibet: Qinghai; N: Shaanxi; SW. Endemic.

Seseli wannienchun (Fu Kuntsun) Pimenov, 1999, Feddes Repert. 110(7-8): 487.

इLibanotis wannienchun Fu Kuntsun, 1981, Fl. Tsinling. 1(3): 458, 409, fig. 351.

Type: CHINA. "Kansu, Tieh-pu Hsien e Kangto ad Chu-li, 05. 08. 1964, Kuo Penchao 5659" (holotype WUK).

Distribution in China: NW: Gansu. Endemic.

Seseli wawrae H. Wolff, 1930, Repert. Spec. Nov. Regni Veg. 27: 315.

$\equiv$ Peucedanum wawrae (H. Wolff) Shan Renhwa, 1982, Fl. Jiangsu 2: 583, fig. 1677, "wawrii". 
Type: CHINA. "Shandung, Chefoo, ravines in hills, 07-08. 1920, Cowdry 757" (lectotype K! barcode K000697461, designated here; isolectotype K! - barcode K000697460); "Kiautschou, Kap Yata, Zimmermann 476" (syntype).

Distribution in China: N: Shandong; NE: Liaoning; Centr.: Jiangsu, Anhui. Endemic.

Seseli yunnanense Franch., 1894, Bull. Annuel Soc. Philom. Paris (sér. 8) 6: 129.

Type: CHINA. "Yun-nan, in pratis circa Kipin-koy, prope Tali, 30. 09. 1886, Delavay 2240" (lectotype P!, designated here; isolectotypes K! barcode K000697463, P! - barcodes P02496419, P02496420, P02496421); "Yun-nan, in collibus calcareis ad Lo-ho-chan, supra Che-tong, prope Tapin-tze, alt. 1500 m, 04. 09. 1885, Delavay 2025" (syntypes P! - barcodes P02496416, P02496417, P02496429, P02496431); "Yun-nan, in pratis montis Pee-ngay-tze, 09. 1882. Delavay 449" (syntype P! barcode P02496423); "Yun-nan, in calcareis montis Hee-chan-men, alt. 3000 m, 03. 09. 1884, Delavay 679, 944" (syntype P! - barcode P02496430); "Yunnan, Tapin-tze, in pratis, 24. 08. 1882, Delavay 455, 2742" (syntypes K! - barcode K000697462, P! - barcode P02496426); "Province de Yun-nan, prairies sèche, des montagnes Mt. Hée Chan Mên (Lan Kong), 03. 09. 1884, Delavay 944” (syntype P! - barcode P02496427).

= Seseli siamicum Craib, 1911, Bull. Misc. Inform. Kew 1911: 59.

Type: THAILAND. "Chiengmai, Eng jungle on Doi Sootep, 300-750 m, 29. 08. 1909, Kerr 774" (lectotype K! - barcode K000697477, designated here; isolectotypes $\mathrm{C}$ ! - barcode C10008567, $\mathrm{K}$ ! barcodes K000697475, K000697476, P! - barcode P02496636, TCD - barcode TCD0017222).

= Seseli mairei H. Wolff, 1930, Repert. Spec. Nov. Regni Veg. 27: 301.

Type: CHINA. "Yunnan, Maire 8"(syntype E! barcode E00265215);"Lou-pou près Tong-tchouan, 08. 1909, Ducloux 1492" (syntype E!); "Lti-lungshan, $8000^{\prime} 28^{\circ} 10^{\prime} \mathrm{N}$, open dry stony pasture, 08 . 1917, Forrest 15179" (syntypes E!, K! - barcode K000687457); "NW flank of the Lichiang Range, $27^{\circ} 30^{\prime}$, open stony pasture, $11000 \mathrm{ft}, 07.1914$, Forrest 12819" (syntypes E!, PE!); "Yunnan, Maire 210" (lectotype E! -barcode E00265186, designated here; isolectotype E!); "Yunnan, Maire 2201" (syntype E! - barcode E00265185); "Yunnan, Maire 2360" (syntype E! - barcode E00265214).

= Ligusticum falcarioides H. Wolff, 1930, Repert. Spec. Nov. Regni Veg. 27: 311.
Type: CHINA. "Yunnan: in pratis montis Hiss (Kiss?)-chong-chang, 2600 m s.l., Maire 3942".

= Peucedanum bupleuriforme H. Wolff, 1934, Repert. Spec. Nov. Regni Veg. 33: 245.

Type: CHINA. "Central-Yünnan: Mountains of the Yangpi river, 08. 1922, Rock 6206" (holotype US; isotype E! - barcode E00265184).

= Peucedanum bupleuroides H. Wolff, 1934, Repert. Spec. Nov. Regni Veg. 33: 245.

Type: CHINA. "Yünnan, in pascuis prope (?) Kin-tchong-chan, 08. 1910, Maire 3486" (holotype P).

Distribution in China: SW: Sichuan, Guizhou, Yunnan; S: Guangxi Zhuang A. R.

Distribution outside China: Laos, Thailand.

Seselopsis tianschanica Schischk., 1950, Bot. Mater. Gerb. Bot. Inst. Komarova Akad. Nauk SSSR 13: 159.

Type: KAZAKHSTAN. "Tian-Schan, jugum Ketmenj-tau in faucibus Kischkine-Czijbut, in rupium fissuris, alt. ca. 2500 m, 14. 08. 1946, Rubtzov" (holotype LE!).

Distribution in Asia: NW: Xinjiang Weiwuer A. R.

Distribution outside China: Kazakhstan.

Silaum silaus (L.) Schinz et Thell., 1915, Vierteljahrsschr. Naturf. Ges. Zürich 60: 359.

$\equiv$ Peucedanum silaus L., 1753, Sp. pl.: 246.

$\equiv$ Selinum silaus (L.) Crantz, 1767, Cl. Umb.

Emend.: 61.

$\equiv$ Ligusticum silaus (L.) Vill., 1779, Prosp. Hist.

Pl. Dauphiné: 25.

$\equiv$ Sium silaus (L.) Roth., 1788, Tent. Fl. Germ. 1: 129 .

$\equiv$ Peucedanum flavum Salisb., 1796, Prodr. Stirp. Chap. Allerton: 163, nom, illeg. (Art. 52.1)

$\equiv$ Crithmum silaus (L.) Wibel, 1799, Prim. Fl.

Werth.: 197.

$\equiv$ Silaus flavescens Bernh., 1800, Syst. Verz.: 174, nom. illeg. (Art. 52.1).

$\equiv$ Cnidium silaus (L.) Spreng., 1813, Neue Schr. Naturf. Ges. Halle 2(1) (Pl. Umb. Prodr.): 40.

$\equiv$ Meum silaus (L.) Baill., 1879, Hist. Pl. 7: 191.

$\equiv$ Silaus silaus (L.) Karst., 1895, Deut. Fl. (ed. 2)

2: 402, nom. illeg. (Art. 23.4).

Type: EUROPE. "In Helvetia, Narbona VIII 73" (lectotype UPS-Burser, designated by Reduron in Jonsell, Jarvis, 2002: 84).

= Peucedanum alpestre L., 1753, Sp. pl.: 246.

= Selinum alpestre (L.) Crantz, 1767, Cl. Umb. Emend.: 60. 
$=$ Silaus alpestris (L.) Besser ex Schult., 1820, in Roemer \& Schultes (eds.), Syst. Veg. (ed. 15 bis) 6 : 36; Besser, 1822, Enum. Pl. Volh.: 43.

= Ligusticum alpestre (L.) Calest., 1905, Webbia 1: 120 .

= Silaus alpestre (L.) Thell., 1926, in Hegi (ed.), Ill. Fl. Mittel.-Eur. 5(2): 1295.

Type: 346.4 (lectotype LINN, designated by J.P. Reduron in Jarvis et al. (2006) Taxon 55(1): 213). $=$ Seseli selinoides Jacq., 1762, Enum. Stirp. Vindob.: 51, 227.

= Silaus selinoides (Jacq.) Halácsy, 1886, in Kerner (ed.), Sched. Fl. Austro-Hung. 4: 37; ex Simonk., 1886, Enum. Fl. Transsilv.: 261.

Type: EUROPE, AUSTRIA. "In pratensibus inter Lanzendorf et Himberg".

$=$ Seseli pratense Crantz, 1767, Stirp. Austr. 3: 96.

= Peucedanum pratense (Crantz) Lam., 1779, Fl.

Franç. 3: 469.

= Silaus pratensis (Crantz) Besser ex Spreng., 1820, in Roemer \& Schultes (eds.), Syst. Veg. (ed. 15 bis) 6: 36 .

$=$ Cnidium pratense (Crantz) Bubani, 1900, Fl. Pyren. 2: 381.

Type: EUROPE, AUSTRIA. "Prope Lantz, Ebersdorf, in prato supra Grunzing".

= Selinum sibiricum Retz., 1781, Observ. Bot. 2: 16.

= Meum sibiricum Spreng., 1813, Neue Schr. Naturf. Ges. Halle 2, 1 (Pl. Umb. Prodr.): 32.

Type: RUSSIA. "Sibir" (holotype B).

= Silaus besseri DC., 1830, Prodr. 4: 161.

= Silaum besseri (DC.) Grossh., 1932, Fl. Kavkaza 3: 170.

Type: EUROPE, UKRAINE. "In campis apricis Podoliae australis [In Osopo campis] 1828, Besser" (lectotype G-DC, designated by Reduron, Jacquemoud (Reduron, 2008: 2367); isolectotype KW-Besser!).

= Silaus gracilis Bunge, 1851, Beitr. Fl. Russl. (Al. Lehmann Reliq. Bot.): 125.

Type: EUROPE, RUSSIA. "Auf der neuen Orenburgschen Linie, Lehmann".

Distribution in China: NW: Xinjiang Weiwuer A. R.

Distribution outside China: Russia, Kazakhstan. Europe.

Sinocarum acronemifolium (C. B. Clarke) P. K. Mukh. et Constance, 1991, Edinburgh J. Bot. 48(1): 43.

$\equiv$ Pimpinella acronemifolia C. B. Clarke, 1879, in Hooker f. (ed.), Fl. Brit. India 2: 686, p.p.
三Acronema acronemifolium (C. B. Clarke) H. Wolff, 1927, in Engler (ed.), Pflanzenreich 90(IV, 228): 332, p.p.

Type: INDIA. "Sikkim, alt. $11.000 \mathrm{ft}$, Chola, Hooker" (holotype K?; isotypes GH!, P!).

Distribution in China: Tibet: Xizang A. R.

Distribution outside China: Myanmar, Nepal, India.

Sinocarum clarkeanum P. K. Mukh. et Constance, 1991, Edinburgh J. Bot. 48(1): 42.

Type: INDIA. "Sikkim, Singaleleh, alt. 10.000 ft, 07. 10. 1870, Clarke 12545"(lectotype K! - barcode K000075375, designated here; isolectotypes E! - barcode E00265189, K!).

Distribution in China: Tibet: Xizang A. R.

Distribution outside China: Nepal, India.

Sinocarum cruciatum (Franch.) H. Wolff [1927, in Engler (ed.), Pflanzenreich 90(IV, 228): 164, nom. inval. (Art. 35.1)] ex Shan Renhwa et Pu Fating, 1985, Fl. Reipubl. Popularis Sin. 55(2): 33.

ECarum cruciatum Franch., 1894, Bull. Annuel Soc. Philom. Paris (sér. 8) 6: 124.

三 Ligusticum cruciatum (Franch.) M. Hiroe, 1958, Umbell. Asia 1: 109.

Type: CHINA. "Yun-nan, in calcareis inter dumeta, ad cacumina montis Ki-chan, prope Tali, alt. 2800 m, 10. 09. 1884, Delavay 182" (lectotype P! barcode P03224861, designated here; isolectotype $\mathrm{P}$ barcodes P02284823, P03224868); Delavay 680 barcodes P02284822, P03224871); "Yun-nan, in dumetis montis Tsang-chan supra Tali, alt. $3000 \mathrm{~m}$, 26. 09. 1884, Delavay 195" (syntype P! - barcodes P03224877, P03224863); "Yun-nan, ad collum Yentze-hay supra Lankong, alt. 3200 m, 20. 10. 1885, Delavay" (syntype P! - barcode P03224878).

= Carum coloratum Diels, 1912, Notes Roy. Bot. Gard. Edinburgh 5: 287.

= Sinocarum coloratum (Diels) H. Wolff, [ 1927, in Engler, Pflanzenreich 90(IV, 228): 164, nom. inval. (Art. 35.1)] ex Shan Renhwa et Pu Fating, 1985, in Fl. Reipubl. Popularis Sin. 55(2): 33, tab.10, fig. $1-4$.

Type: CHINA. "Yunnan, grassy dry ledges of cliffs on the eastern flank of the Lichiang Range. Lat. $27^{\circ} 25^{\prime}$ N. Alt. 12-13000 ft, 08. 1906, Forrest 2874" (syntypes BM! - barcode BM000574893, E! barcode E00000375, P! - barcode P03224806); "Grassy ledges of cliffs on the eastern flank of the Lichiang Range. Lat. $27^{\circ} 25^{\prime}$ N. Alt. 11-12000 ft, 09. 1906, Forrest 3060" (lectotype E!, designated by Watson (1998: 382); isolectotype BM - barcode BM0000574892). 
= Sinocarum pseudocruciatum H. Wolff, 1929, Repert. Spec. Nov. Regni Veg. 27: 182, nom. inval. (Art. 35.1).

Type: CHINA. "Szechwan, N. Wuschang, 03. 1889, Henry 7067" (lectotype K! - barcode K000685660, designated here; isolectotype LE!).

Distribution in China: Tibet: Xizang A. R.; SW: Sichuan, Yunnan.

Distribution outside China: Myanmar, India.

Sinocarum cruciatum (Franch.) H. Wolff var. linearilobum (Franch.) Shan Renhwa et Pu Fating, 1985, in Shan Renhwa \& Shen Menglan (eds.), Fl. Reipubl. Popularis Sin. 55(2): 35.

$\equiv$ Carum cruciatum Franch. var. linearilobum Franch., 1894, Bull. Annuel Soc. Philom. Paris (sér. 8) 6: 124 .

Type: CHINA. "Yunnan, in dumetis montis Maeul-chan, prope cacumina, alt. $3500 \mathrm{~m}, 10.09 .1889$, Delavay 3899" (syntypes P! - barcodes P03224862, P03224869, P03224870, K!).

= Sinocarum caespitosum H. Wolff, 1929, Repert. Spec. Nov. Regni Veg. 27: 181, nom. inval (Art. 35.1)

= Carum forrestii M. Hiroe, 1979, Umbelliferae World: 872.

Type: CHINA. "Yunnan, western flank of the Shweli-Salwin divide, 9000 ft. s.1. Lat. 25²0', 08. 1912, Forrest 8986" (holotype E! - barcode E000004537).

Distribution in China: Tibet: Xizang A. R.; SW: Yunnan. Endemic.

Sinocarum dolichopodum (Diels) H. Wolff [1927, in Engler (ed.), Pflanzenreich 90(IV, 228): 164, nom. inval. (Art. 35.1)] ex Pu Fating, 1984, in Wu Zhengyi (ed.) Index Fl. Yunnan. 1: 928.

$\equiv$ Carum dolichopodum Diels, 1912, Notes Roy. Bot. Gard. Edinburgh 5: 287.

Type: CHINA. "Yunnan, shady ledges on and at the base of cliffs on the eastern flank of the Lichiang Range. Lat. $27^{\circ} 25^{\prime}$ N. Alt. 11-12000 ft, 08. 1906, Forrest 2872" (lectotype E!, designated here; isolectotypes BM! - barcode BM000551439, P! barcode P03224926).

= Ligusticum rockii M. Hiroe, 1958, Umbell. Asia 1: 110.

Type: CHINA. "Western Szechuan, China. Mount Mitzuga, west of Muli Gomba, alt. 30504875 meters. Itla, 06. 1928, Rock 16541" (holotype UC! - barcode UC938819; isotypes A - barcode A00076821, US! - barcode US00731242).

Distribution in China: SW: Sichuan, Yunnan. Endemic.
Sinocarum filicinum H. Wolff, 1929, Repert. Spec. Nov. Regni Veg. 27: 182, nom. inval. (Art. 35.1).

$\equiv$ Carum chinense M. Hiroe, 1979, Umbelliferae World: 872.

Type: CHINA. "Yunnan, eastern flank of the Tali Range, 2540 m s.1., 08. 1913, Forrest 7230, 11691" (syntypes BM! - barcode BM000551440, E! barcode E00000439, K! - barcodes K000685661, K000685662, PE!); "Yunnan, eastern flank of the Tali Range, 2540 m s.l., Forrest 6963" (lectotype E!, designated by M. Farille in E; isolectotype K! barcode K000685663).

Distribution in China: Tibet: Xizang A. R.; SW: Sichuan, Yunnan. Endemic.

Sinocarum minus M. F. Watson, 1996, Edinburgh J. Bot. 53(1): 140, fig. 5, E-H.

$\equiv$ Acronema minus (M. F. Watson) M. F. Watson et Pan Zehui, 2004, Acta Phytotax. Sin. 42(6): 561.

Type: NEPAL. "Arun Valley, Barun Khola, N of Num, 12500 ft, 07. 06. 1956, Stainton 547" (holotype $\mathrm{BM}$ !; isotype $\mathrm{E}$ - barcode $\mathrm{E} 00018059$ ).

- Acronema wolffianum auct. non Fedde ex H. Wolff: Liou Shoulu et Shan Renhwa, 1980, Acta Phytotax. Sin. 18(2): 199.

Distribution in China: Tibet: Xizang A. R.; SW: Yunnan.

Distribution outside China: Bhutan, Nepal.

Sinocarum pauciradiatum Shan Renhwa et $\mathrm{Pu}$ Fating, 1980, Acta Phytotax. Sin. 18(3): 375.

Type: CHINA. "Xizang: Cona Xian, alt. 4300$4500 \mathrm{~m}, 17$. 07. 1975, Wu Zhengyi 75-1021" (holotype PE; isotype KUN!).

Distribution in China: Tibet: Xizang A. R.; SW: Sichuan, Yunnan.

Distribution outside China: Bhutan, Nepal, India.

Sinocarum pityophilum (Diels) H. Wolff [1927, in Engler (ed.), Pflanzenreich 90(IV, 228): 166, nom. inval. (Art. 35.1)] ex Pu Fating, 1984, Index Fl. Yunnan. 1: 928.

$\equiv$ Carum pityophilum Diels, 1912, Notes Roy. Bot. Gard. Edinburgh 5: 228.

Type: CHINA. "NW Yunnan, China: open, dry situations in pine scrub on the eastern flank of the Lichiang Range. Lat $27^{\circ} 15^{\prime}$ N. Alt. 10-11000 ft, 10. 1906, Forrest 3078" (lectotype E!, designated here; isolectotypes BM! - barcode BM000813369, P!).

Distribution in China: SW: Yunnan. Endemic. 
Sinocarum schizopetalum (Franch.) H. Wolff [1927, in Engler (ed.), Pflanzenreich 90(IV, 228): 167, nom. inval. (Art. 35.1)] ex Pu Fating, 1985, Fl. Reipubl. Popularis Sin. 55(2): 33, tab. 11, fig. 1-5.

$\equiv$ Carum schizopetalum Franch., 1894, Bull. Annuel Soc. Philom. Paris (sér.) 8(6): 118.

$\equiv$ Dactylaea schizopetala (Franch.) Wu Zhengyi, 1984, Index Fl. Yunnan. 1: 910.

Type: CHINA. "Yun-nan, in locis adumbratis inter muscos, ad saxa, praesertim in monte Tsangchan ad cacumina supra Tali, alt. $4000 \mathrm{~m}, 25.07$. 1884, Delavay 196"(lectotype P!, designated here; isolectotypes G, K! - barcode K000685665, P); "Yun-nan, in monte Tsang-chan ad cacumina supra Tali. Alt. 4000 m, 29. 08. 1889, Delavay 4018 “(syntypes K! - barcode K001091308, P!).

Distribution in China: Tibet: Xizang A. R.; SW: Yunnan.

Distribution outside China: Myanmar.

Sinocarum schizopetalum (Franch.) H. Wolff var. bijiangense (Liou Shoulu) Liu Xintian, 1997, in Wu Zhengyi (ed.), Fl. Yunnan. 7: 521.

$\equiv$ Sinocarum bijiangense Liou Shoulu, 1990, Acta Phytotax. Sin. 28(2): 151.

Type: CHINA. "Yunnan: Bijiang, $4800 \mathrm{~m}$ alt., 11. 09. 1964, S. K. Wu 8738" (holotype KUN).

Distribution in China: SW: Yunnan. Endemic.

Sinocarum shanii Pimenov et Kljuykov, 2006, Komarovia 4: 123.

三Dactylaea wolffiana Fedde ex H. Wolff, 1930, Repert. Spec. Nov. Regni Veg. 27: 304.

三 Sinocarum wolffianum (Fedde ex H. Wolff)

Shan Renhwa et Pu Fating, 1993, in Wang Wentsai (ed.), Vasc. Pl. Hengduan Mount.: 1312, nom. illeg. (Art. 53.1), non P. K. Mukh. et Constance (1991).

Type: CHINA. "Tibet, 30. 08. 1919, KingdonWard 3601" (holotype E!).

Distribution in China: SW: Yunnan.

Distribution outside China: Myanmar.

Sinocarum sikkimense (P. K. Mukh.) P. K. Mukh. et Constance, 1991, Edinburgh J. Bot. 48(1): 43.

三 Acronema sikkimense P. K. Mukh., 1978, in Cauwet-Marc et Carbonier, Les Ombellifères, Contr. Pluridisc. Syst.: 52, "sikkimensis".

Type: INDIA. "Sikkim, Lachoong, 3650-4000 m, Hooker" (holotype CAL; isotype K!).

Distribution in China: Tibet: Xizang A. R.

Distribution outside China: Bhutan, Nepal, India.
Sinocarum vaginatum $\mathrm{H}$. Wolff, 1929, Repert. Spec. Nov. Regni Veg. 27: 183, nom. inval. (Art. 35.1).

= Carum vaginatum (H. Wolff) M. Hiroe, 1979, Umbelliferae World: 871.

Type: CHINA. "Yunnan, West China: Ghi Shan $25^{\circ} 55^{\prime} \mathrm{N}$, on the open pasture $11000 \mathrm{ft}, 08.1917$, Forrest 15484" (lectotype E!, designated here; isolectotype K! - barcode K000685664).

Distribution in China: Tibet: Xizang A. R., Qinghai; SW: Sichuan, Yunnan. Endemic.

Sinocarum wolffianum (Fedde ex H. Wolff) P. K. Mukh. et Constance, 1979, Edinburgh J. Bot. 48(1): 42.

$\equiv$ Acronema wolffianum Fedde ex H. Wolff, Repert. Spec. Nov. Regni Veg. 27: 328. 1930.

$\equiv$ Pimpinella feddeana M. Hiroe, 1979, Umbelliferae World: 832.

Type: INDIA. "Himalaya, Sikkim, Jongri, $12000 \mathrm{ft} ., 15.10 .1875$, Clarke 25796" (lectotype K!, designated by Mukherjee, Constance, 1991: 42).

Distribution in China: SW: Yunnan.

Distribution outside China: Bhutan, Nepal, India.

Sinodielsia cuneata ( $\mathrm{H}$. Wolff) Pimenov et Kljuykov, 1999, Feddes Repert. 110(7-8): 489, fig. $1, \mathrm{E}, \mathrm{F}$.

三 Physospermopsis cuneata H. Wolff, 1929, Repert. Spec. Nov. Regni Veg. 27: 126.

Type: CHINA. "Yunnan, Yangtze watershed, prefectural district of Likiang, eastern slopes of Likiang Snow Range, 11000-13000 feet, 16. 08. 1922, Rock 5854" (lectotype P!, designated here; isolectotypes E! - barcode E00000217, GH barcode GH00077443, US).

Distribution in China: SW: Sichuan, Yunnan. Endemic.

Sinodielsia delavayi (Franch.) Pimenov et Kljuykov, 1999, Feddes Repert. 110(7-8): 488.

$\equiv$ Peucedanum delavayi Franch., 1894, Bull. Annuel Soc. Philom. Paris (sér.) 8(6): 143.

Type: CHINA. "Yun-nan, in rupibus calcareis ad fauces, montis Pee-cha-ho, prope Mo-so-yn [Dans les roches calcairs, aux gorges de Pee cha ho, près de Mo-so-yn (Lan Kong)], 31. 08. 1884, Delavay 945" (lectotype P! - P02272001, designated here; isolectotype P! - barcodes P00834661, P02272004).

= Sinodielsia yunnanensis H. Wolff, 1925, Notizbl. Bot. Gart. Berlin-Dahlem 9: 278. 
$=$ Meeboldia yunnanensis $(\mathrm{H}$. Wolff) Constance et Pu Fating, 1997, in Wu Zhengyi (ed.), Fl. Yunnan. 7: 405, tab. 109, fig. 1-5.

Type: CHINA. "Yunnan, reg. bor., Yunnan-fu, Cavalerie 422" (holotype P!).

= Physospermopsis cruciata H. Wolff, 1929, Repert. Spec. Nov. Regni Veg. 27: 127.

$=$ Pleurospermum cruciatum $(\mathrm{H}$. Wolff) $\mathrm{M}$. Hiroe, 1979, Umbelliferae World: 747.

Type: CHINA. "Yunnan, region of Tungshan, Yangtze drainage bassin, east of Likiang, 1923, Rock 10499" (lectotype E! - barcode E00000236, designated here; isolectotypes UC!, US!).

= Physospermopsis forrestii Fedde ex H. Wolff, 1929, Repert. Spec. Nov. Regni Veg. 27: 179.

Type: CHINA. "Yunnan, West China: MekongSalwin divide, Lat. $28^{\circ} 10^{\prime}$ N, 09. 1914, Forrest 13263" (lectotype E! - barcode E00000235, designated here; photo BM).

Distribution in China: Tibet: Xizang A. R.; SW: Sichuan, Yunnan. Endemic.

Sinodielsia microloba Kljuykov, 1986, Feddes Repert. 97(11-12): 757, fig. 1.

Type: CHINA. "South-Eastern Tibet, Kongbo province, Kyabden, Nyang Chu, $10.000 \mathrm{ft}, \mathrm{N} 29^{\circ} 40^{\prime}$, E 94¹6', 31. 08. 1938, Ludlow, Sherriff, Taylor 6201" (holotype BM! - barcode BM000885492; isotype E! - barcode E00000231).

Distribution in China: Tibet: Xizang A. R. Endemic.

Sinodielsia thibetica (H. Boissieu) Kljuykov et P. K. Mukh., 1991, Feddes Repert. 102(5-6): 383.

$\equiv$ Vicatia thibetica H. Boissieu, 1906, Bull. Soc. Bot. France 53: 423.

Type: CHINA. "Thibet oriental, province de Batang, Yargong, haies, lieux ombrages et forêts, 06. 08. 1903, Soulié 3205C" (lectotype P! - barcode P00834493, designated here; isolectotypes P! barcodes P00834492, P00834494).

Distribution in China: Tibet: Xizang A. R., Qinghai; SW: Sichuan, Yunnan. Endemic.

Sinolimprichtia alpina H. Wolff, 1922, Repert. Spec. Nov. Regni Veg. Beih. 12 (Limpr. Bot. Reise China Ost-Tibet): 449.

Type: CHINA. "Ost-Tibet: Beju-Batang, Kammhole des Pass Mala, 4600 m, 13.08.1914. Limpricht 2191" (lectotype WU! - barcode WU00043687, designated here; isolectotypes B-destr., BM - photo). Type specimen in WU is represented by very scanty material ("kleptotype") - a fragment of leaf and a umbelet in pocket. Probably an epitypification is needed.

= Sinolimprichtia alpina $\mathrm{H}$. Wolff var. dissecta Shan Renhwa et Liou Shoulu, 1979, in Shan Renhwa \& Sheh Menglan (eds.), Fl. Reipubl. Popularis Sin. 55(1): 192, 299, tab. 101, fig. 9.

Type: CHINA. "Yunnan, Teh-Ching Hsien, A-tun-tze, alt. $3600 \mathrm{~m}, 03-05.08 .1935$, C. W. Wang 64924" (holotype NAS!).

Distribution in China: Tibet: Xizang A. R., Qinghai; SW: Sichuan, Yunnan. Endemic.

Sium medium Fisch. et C. A. Mey., 1843, Index Sem. Horti Petrop. 9, Suppl.: 19.

$\equiv$ Drepanophyllum medium (Fisch. et C. A. Mey.) Koso-Pol., 1914, Trudy Tiflissk. Bot. Sada 16: 181.

Type: KAZAKHSTAN. "In humidis regionum altaicerum atque in desertis songoro-kirgisicis, 07. 1826, Ledebour, Meyer 269" (holotype LE!).

= Sium latijugum C. B. Clarke, 1879, in Hooker f. (ed.), Fl. Brit. India 2: 683.

Type: INDIA. "Kashmir, alt. $5000-7000 \mathrm{ft}$, Thomson 300" lectotype K! - barcode K000685623, designated here; isolectotypes MANCH!, P!).

Distribution in China: NW: Xinjiang Weiwuer A. R.

Distribution outside China: India, Pakistan, Kazakhstan, Kirghizia, Tajikistan, Uzbekistan, Afghanistan.

Sium sisaroideum DC., 1830, Prodr. 4: 124.

三Sisarum sisaroideum (DC.) Schischk., 1935, in Krylov, Fl. Zapadnoi Sibiri 8: 2077.

$\equiv$ Pimpinella sisaroidea (DC.) Manden., 1950, in Charadze (ed.), Fl. Georgiae 6: 425.

$\equiv$ Sium sisarum L. subsp. sisaroideum (DC.) Soó, 1978, Acta Bot. Acad. Sci. Hung. 23(3-4): 380 [1977].

Type: IRAN. "In arenosis umbrosis circa Badalan, Distr. Khoi, Prov. Aderbeizan, Persiae, 20. 07. 1828, Szovits 561" (lectotype G-DC!, designated here; isolectotype LE!).

= Sium lancifolium M. Bieb., 1819, Fl. Taur.Caucas. 3: 230, nom. illeg. (Art. 53.1), non Schrank (1789).

= Berula lancifolia (M. Bieb.) Besser, 1822, Enum. pl. Volh.: 44.

=Pimpinella sisarum (L.) Koso-Pol. var. lancifolia (M. Bieb.) Koso-Pol., 1914, Bull. Soc. Imp. Naturalistes Moscou, n.s. 28: 180.

= Sium sisarum L. var. lancifolium (M. Bieb.) Thell., 1926, in Hegi (ed.), Ill. Fl. Mitt.-Eur. 5(2): 1233. 
Type: UKRAINE. "In paludosis inter virgulta salicina et Opulum. per Rossiam australioram, usque Ucrainiam [Tanais]. 1816. Henning" (lectotype LEBieb!, designated by N. M. Fedoronchuk in LE).

= Sium lancifolium M. Bieb. var. elongatum Parsa, 1948, Kew Bull. 1948: 200.

Type: IRAN. "N Persia, hills above Gilas, 657500 ft, 29. 05. 1929, Parsa".

- Sium sisarum auct. non L.: Ledeb., 1829, Fl. Alt. 1: 351 .

- Sium latifolium auct. non L.: Wang Tiehteng, 1985, in Shan Renhwa \& Sheh Menglan (eds.), Fl. Reipubl. Popularis Sin. 55(2): 157, tab. 65, fig. 7-9, p.p. A. R.

Distribution in China: NW: Xinjiang Weiwuer

Distribution outside China: Russia, Kazakhstan, Kirghizia, Tajikistan, Uzbekistan, Turkmenistan, Afghanistan, Iran, Azerbaijan, Georgia, Armenia, Turkey, Iraq, Europe.

Sium suave Walter, 1788, Fl. Carol.: 115.

Type: NORTH AMERICA, USA. "Carolina, Walter" (holotype US?).

= Sium cicutifolium Schrank, 1789, Baier. Fl. 1: 558.

= Apium cicutifolium (Schrank) Benth. et Hook. f. ex Forbes et Hemsl., 1887, J. Linn. Soc. Bot. 23: 328.

Type: RUSSIA. "Nascitur ab Obo fluvis in omno ulteriori Sibiria tam humidis quam siccis locis".

= Sium lineare Michx., 1803, Fl. Bor--Amer. 1: 167.

= Drepanophyllum lineare (Michx.) Koso-Pol., 1914, Bull. Soc. Imp. Naturalistes Moscou, s.n. 28: 182.

Type: NORTH AMERICA, CANADA. "In aquosis Canadae".

= Critamus dauricus Hoffm., 1816, Gen. pl. Umbell. (ed. 2): 182.

= Cicuta daurica (Hoffm.) Fisch. ex Schult., 1820 , in Roemer \& Schultes (eds.), Syst. veg. (ed. 15 bis) 6: 453 .

= Falcaria daurica (Hoffm.) DC., 1830,Prodr. 4: 110, "dahurica".

Type: Probably described on the basis of plants from Hort. Gorenk.

= Sium nipponicum Maxim., 1873, Bull. Acad. Petersb. 19: 286.

= Sium suave Walter var. nipponicum (Maxim.) H. Hara, 1952, J. Fac. Sci. Univ. Tokyo (sect. 3 Bot.) 6(2): 95.

Type: JAPAN. "In Nippon media, probabiliter non procul a Yokohama [Japonia, Nippon media],
1866, Tschonoski [Sugawa]" (lectotype LE!, designated by Vinogradova, 2004: 31; isolectotypes GH - barcode GH00348374, K!, P!).

= Sium formosanum Hayata, 1921, Icon. P1. Formosan. 10: 16, fig. 9.

Type: CHINA. "[Taiwan] Atamu, 08. 1905, Nakahara 932" (lectotype TI!, designated here; isolectotype TAIF).

Distribution in China: N: Neimenggu A. R., Shaanxi, Shanxi, Hebei, Shandong; NE: Heilongjiang, Jilin, Liaoning; Centr.: Henan, Jiangsu, Anhui, Hubei, Jiangxi, Zhejiang; SW: Guizhou; S: Taiwan.

Distribution outside China: Russia, Japan, Korea, Mongolia. N. America.

Sium tenue (Kom.) Kom., 1916, Izv. Imp. S.Peterburgsk. Bot. Sada 16(1-2): 174.

三Sium cicutifolium Schrank var. tenue Kom., 1907, Trudy Imp. S.-Peterburgsk. Bot. Sada 25 (Fl. Mansh. 3): 150.

Type: KOREA. "Species pratiis montanis humidis proprius, example gratia, marginis planities basalticis Peishan [Prope pag. Karami]. 22. 06. 1897. Komarov" (lectotype LE!, designated here; isolectotype LE!).

= Sium turfosum H. Wolff, 1920, Repert. Spec. Nov. Regni Veg. 17: 172.

Type: KOREA. "Hallaisan, in turfosis, $1700 \mathrm{~m}$ s.1., 10. 1907, Taquet 114" (lectotype E!, designated here; isolectotypes BM - barcode BM000944757, LE!).

Distribution in China: NE: Heilongjiang.

Distribution outside China: Russia, Korea.

Sium ventricosum (H. Boissieu) Wang Lisong et M. F. Watson, 2016, Phytotaxa 288(3): 266.

$\equiv$ Apium ventricosum $\mathrm{H}$. Boissieu, 1906, Bull. Soc. Bot. France 53: 425.

$\equiv$ Sinocarum ventricosum $(\mathrm{H}$. Boissieu) Pimenov et Kljuykov, 2003, Feddes Repert. 114: 356.

Type: CHINA. "Zogalougla, marécages. Blanche 2613" (holotype P! - barcode P00279520).

= Sium frigidum Hand.-Mazz., 1933, Symb. Sin. 7(3): 719, tab. 11, fig. 2-4.

= Chamaesium frigidum (Hand.-Mazz.) Shan Renhwa, 1993, in Wang Wentsai (ed.), Vasc. Pl. Hengduan Mount.: 1290.

Type: CHINA. "Prov. Yunnan bor.-occid.: supra vicum Anangu austro-orientalem pagi Dschundien (Chungtien), in regione frigidae temperatae limo turfi Diolo, 16. 08. 1915, Handel-Mazzetti 7684" (lectotype WU! - barcode WU0025978, designated 
here; isolectotypes E! - barcode E00000562, GH barcode GH00062614, LE!, US).

Distribution in China: SW: Sichuan, Yunnan. Endemic.

Sphallerocarpus gracilis (Besser ex Trev.) KosoPol., 1916, Bull. Soc. Imp. Naturalistes Moscou, s.n. 29: 201.

$\equiv$ Chaerophyllum cyminum Besser, 1821, Ind. Sem. Horti Wratisl. App. 3: 1, nom. inval.

$\equiv$ Chaerophyllum gracile Besser ex Trev., 1826, Nova Acta Phys.-Med. Acad. Caes. Leop.-Carol. Nat. Cur. 13(1): 172.

$\equiv$ Myrrhis gracilis (Besser ex Trev.) Spreng., 1827, Syst. Veget. (ed. 16) 4(2): 120.

$\equiv$ Sphallerocarpus cyminum Besser ex DC., 1829, Col. Mém. 5 (Mém. Fam. Ombell.): 60.

$\equiv$ Conopodium cyminum (Besser ex DC.) Benth. et Hook. f. ex B. D. Jacks., 1893, Index Kew. 1(1): 597.

Type: Plants cultivated in Breslau (Wrozlav) bot. gard. from the seeds obtained from F. Fischer (De loco natali nil nobis innotuit).

Distribution in China: NW: Xinjiang Weiwuer A. R., Gansu; Tibet: Qinghai; N: Neimenggu A. R., Ningxia Huizu A. R., Shanxi, Hebei; NE: Heilongjiang, Jilin, Liaoning; SW: Sichuan, Yunnan.

Distribution outside China: Russia, Korea, Mongolia.

Note: About correct name of the species see also: Turner, 2016, Ann. Bot. Fennici 53: 307.

Spuriopimpinella arguta (Diels) He Xingjin et Wang Zhixin, 2013, Nordic J. Bot. 32(5): 653.

$\equiv$ Pimpinella arguta Diels, 1900, Bot. Jahrb. Syst. 29 (3-4) (Fl. Centr. China): 496.

Type: CHINA. "Prov. Szechuan, N Wushan [Die Mittelgebirge des Yangtze-Durchbruchs], 1885-1888, Henry 7086" (lectotype K! - barcode K000685536, designated here; isolectotypes B, E! barcode E00000472, GH - barcode GH00077444, P! - barcodes P00752712, P00834755).

Distribution in China: NW: Gansu; N: Shanxi, Hebei; Centr.: Henan, Anhui, Hubei, Hunan; SW: Sichuan, Guizhou. Endemic.

Spuriopimpinella brachystyla (Hand.-Mazz.) Kitag., 1941, J. Jap. Bot. 17(10): 559.

$\equiv$ Pimpinella brachystyla Hand.-Mazz., 1933, Oesterr. Bot. Z. 82: 251.

Type: CHINA. "China bor.: Prov. Schansi, in monte Ta-Wutai-schan, ad confines prov. Tschili [Schansi-Tschili-Grenze: Ta-Wutai-schan], 27. 07. 1929, Serre 2382" (holotype W!).
= Pimpinella nakaiana Kitag. ex Nakai et Kitag., 1934, Rep. First Sci. Exped. Manchoukuo, sect. IV, 1: 39 , tab. 13.

Type: CHINA. "In umbrosis sylvaticis montis prope Chang-shan-yii, 13. 09. 1933, Nakai, Honda, Kitagawa" (holotype TI?).

Distribution in China: NW: Gansu; N: Neimenggu A. R., Shaanxi, Shanxi, Hebei; NE; Centr.: Anhui. Endemic.

Spuriopimpinella calycina (Maxim.) Kitag., 1941, J. Jap. Bot. 17(10): 559.

三Pimpinella calycina Maxim., 1873, Bull. Acad. Imp. Sci. Saint-Petersbourg 19: 182.

三 Carum calycinum (Maxim.) Koso-Pol., 1916, Bull. Soc. Imp. Naturalistes Moscou, s.n. 29: 199.

三Aegopodium calycinum (Maxim.) Vorosch., 1966, Fl. Sovetsk. Dal'nego Vost.: 319.

Type: JAPAN. "Japonia, [in jugi Kundshosan silvis vetustis frondosis] Nagasaki, Kundsho-san, 29. 09. 1863, Maximowicz" (lectotype LE!, designated by Grubov (Vinogradova, 2004: 30); isolectotypes C! - barcodes C10008517, C10008518, FI!, GH, GOET, M! - barcode M0172942, NY - barcode NY00406193, P! - barcode P00752687, S! barcode S-G-4891, US! - barcode US00127212); "Japonia, Jokohama, 12/24. 08. 1862, Maximowicz" (syntypes FI!, GOET - barcode GOET011401, LE!, K - barcode K000685537, S! - barcode S-G4892); "Japonia, Nagasaki, Simabara, 09/21. 09. 1863, Maximowicz" (syntypes LE!, M - barcode M0172943); "Japonia, Nagasaki, Naga yama, 21. 09. 1863, Maximowicz" (syntypes LE!, GH - barcode GH00077445, NY - barcode NY00406192); "Japonia, Nippon, prov. Nambu, 1865, Tschonoski" (syntype LE!); "Nippon media. 1866. Tschonoski" (syntype P - barcode P02496734).

= Pimpinella calycina Maxim. var. brachycarpa Kom., 1907, Trudy Imp. S.-Peterburgsk. Bot. Sada 25(1) (Fl. Mansh. 3): 145.

= Pimpinella brachycarpa (Kom.) Nakai, 1909, Tyosen-syokubutu (Fl. koreana) 1: 261.

= Spuriopimpinella brachycarpa (Kom.) Kitag., 1941, J. Jap. Bot. 17(10): 559.

= Aegopodium brachycarpum (Kom.) Schischk., 1950, in Schischkin (ed.), Fl. URSS 16: 457.

Type: KOREA. "Syntypi plures: Via inter Niigata et Omoso, trajectum Santa-Alin, 24. 07. 1896, Komarov 1168" (syntypes FI!, LE!, K!); "I-schesun-che, 04. 08. 1896, Komarov 1168" (syntype LE!); "Loe-lin, Korea septentrionalis, ad fl. Jalu, ad mont Ilan-jen, pr. Nareni-jun, 06. 07. 1897, Komarov" (syntype LE!); "Seselin-Korani; Czatep-uen Komarov" (syntype LE?); "Korachi; pr. opp. Samsu 
Peksan-jen, 04-05. 08. 1897, Komarov" (syntype LE!); "vall. Jalu, pag. Schi-cy-da-gon; Undon-ien, Komarov" (syntype LE?); "fl. Jalu, prope pag. Pantentzi-gou; Po-pen. Komarov" (syntype LE?).

= Pimpinella brachycarpa (Kom.) Nakai var. hallaisanensis Lee Woo Tchul et Jang Geun Jing, 1995, Korean J. Pl. Taxon. 25(1): 8.

$=$ Pimpinella hallaisanensis (Lee Woo Tchul et Jang Geun Jing) Jang Geun Jing, Paik Weon Ki et Lee Woo Tchul, 1999, Korean J. Pl. Taxon. 29(2): 163.

Type: KOREA. "Cheju prov., prope vallis Minjangol, montis Hallosan, $650 \mathrm{~m}$ alt., 17. 07. 1991, Jang Geun Jing” (holotype Kangwan Univ.).

Distribution in China: N: Hebei; NE: Heilongjiang, Jilin, Liaoning.

Distribution outside China: Russia, Japan, Korea.

Spuriopimpinella komarovii Kitag., 1941, J. Jap. Bot. 17(10): 560.

$\equiv$ Pimpinella komarovii (Kitag.) T.Lee, 1969, Seoul Univ. J. (B) 20: 164.

Type: KOREA. "Prov. Kan-nan: in monte Unzen-rei, 16. 08. 1935, Nakai 15608" (neotype TI!, designated here).

- Pimpinella arguta Nakai, 1911, Tyosen-syokubutu (F1. Koreana) 1: 399, fig. 501, nom. illeg., non Diels (1900).

Distribution in China: N: Hebei; NE: Heilongjiang, Liaoning.

Distribution outside China: Korea.

Spuriopimpinella koreana (Yabe) Kitag., 1941, J. Jap. Bot. 17(10): 560.

$\equiv$ Pimpinella nikoensis Yabe ex Hisauti var. koreana Yabe, 1903, Bot. Mag. (Tokyo) 17 (196): 106.

$\equiv$ Pimpinella koreana (Yabe) Nakai, 1909, Tyosen-syokubutu (Fl. koreana) 1: 261.

Type: KOREA. "Kang-uöng-do, in monte Keum-gang-san, Corea, 15. 08. 1902, Uchiyama".

Distribution in China: Centr.: Zhejiang.

Distribution outside China: Korea.

Stenocoelium athamantoides (M. Bieb.) Ledeb., 1829, Fl. Altaic. 1: 298.

$=$ Cachrys athamantoides M. Bieb., 1819, Fl. Taur.-Caucas. 3: 217, sine typo indicato.

$=$ Rumia athamantoides (M. Bieb.) DC., 1830, Prodr. 4: 98.

Type: RUSSIA. "Altai, in lapidosis alpinis ad fl. Tschuja contra ostium fl. Tschagan locis lapidosis [in alpibus contra ostium fluvii Tschagan locis lapidosis], VII.1826, Bunge 227" (lectotype LE!, designated by Vinogradova (2005: 966); isolectotype LE!).

Distribution in China: NW: Xinjiang Weiwuer A. R.

Distribution outside China: Russia, Mongolia, Kazakhstan.

Stenocoelium depressum (Shan Renhwa et Sheh Menglan) Pimenov et Kljuykov, comb. nov.

$\equiv$ Libanotis depressa Shan Renhwa et Sheh Menglan, 1983, Acta Phytotax. Sin. 21(1): 82, tab. 2, fig. 1-5.

$\equiv$ Seseli depressum (Shan Renhwa et Sheh Menglan) V. M. Vinogr., 1989, Novosti Sist. Vyssh. Rast. 26: 124.

Type: CHINA. "Qinghai: Nangqen, 07. 1965, Y. C. Yang 1395" (holotype NAS).

Distribution in China: Tibet: Xizang A. R., Qinghai; SW: Sichuan. Endemic.

Stenocoelium popovii V. M. Vinogr. et Fedor., 1979, Novosti Sist. Vyssh. Rast. 16: 148, fig. 1.

Type: CHINA. "Dshungaria, Tian-Schan, distr. Urumtschi, pag. Biangou, in superiore fl. Tasenku, zona Picea, in rupibus aridis australibus, 25. 09. 1929, Popov 276" (holotype LE!).

Distribution in China: NW: Xinjiang Weiwuer A. R. Endemic.

Stenocoelium trichocarpum Schrenk, 1841, Enum. Pl. Nov. 1: 80; 1843, Bull. Phys.-Math. Acad. Petersb. 1: 80.

$\equiv$ Seseli trichocarpum (Schrenk) B. Fedtsch., 1915, Rastit. Turkest.: 616.

Type: KAZAKHSTAN. "Songoria, in montibus Tarbagatai, 25. 08. 1841, Schrenk 963" (lectotype LE!, designated here; isolectotypes AA!, MANCH!, $\mathrm{P}$ !).

Distribution in China: NW: Xinjiang Weiwuer A. R.

Distribution outside China: Kazakhstan.

Tetrataenium olgae (Regel et Schmalh.) Manden., 1959, Trudy Tbilis. Bot. Inst. 20: 8.

EHeracleum olgae Regel et Schmalh., 1881, Izv. Obšč. Ljubit. Estestv. 34, 2 (Descr. P1. Nov. Rar. Fedtsch.): 38.

$\equiv$ Pastinaca olgae (Regel et Schmalh.) KosoPol., 1916, Bull. Soc. Imp. Naturalistes Moscou, s.n. 29: 113, nom. inval., non Regel et Schmalh. (1882).

$\equiv$ Platytaenia olgae (Regel et Schmalh.) Korovin, 1959, in Vvedensky (ed.), Fl. Uzbekistan. 4: 465. 
Type: KIRGHIZIA. "In Kokaniae trajectum Isfairam [In descendi trajectis Isfairam in Alai], 8000' alt., 20. 07. 1871, O. A. Fedtschenko" (lectotype LE!, designated by Vinogradova, 2001: 51). TAJIKISTAN. "In valle fl. Sarawschan inter Obburdon et Pachud, alt. 5-6000', O. A. Fedtschenko" (syntype LE!); "In montibus Naubod prope Varsaminor, alt. 4500-8000', O. A. Fedtschenko" (syntype LE!).

= Heracleum polyadenum Rech. f., 1963, K. Danske Vid. Selsk., Biol. Skrift. 13, 4 (Symb. Afghan. 5): 85 .

Type: AFGHANISTAN. "Munjan: Sanglich, 3450 m, 24. 07. 1937, Koelz 12617" (holotype US! barcodes US00289210, US00289211; photo W!).

- Heracleum nubigenum auct. non C. B. Clarke: M. Hiroe, 1958, Umbell. Asia 1: 191.

A. R.

Distribution in China: NW: Xinjiang Weiwuer

Distribution outside China: Pakistan, Kirghizia, Tajikistan, Uzbekistan, Afghanistan.

Tilingia ajanensis Regel, in Regel et Tiling, 1859, Nouv. Mém. Soc. Imp. Naturalistes Moscou 11 (Fl. Ajan.): 97.

三Selinum tilingia Maxim., 1886, Bull. Acad. Imp. Sci. Saint-Petersbourg 31: 50, nom. illeg.

$\equiv$ Cnidium ajanense (Regel et Tiling) Drude, 1898, in Engler \& Prantl (eds.), Nat. Pflanzenfam. 3(8): 210 .

$\equiv$ Cnidium tilingia (Maxim.) Takeda, 1906, Bot. Mag. (Tokyo) 20: (305), fig. 2, nom. illeg.

$\equiv$ Ligusticum ajanense (Regel et Tiling) KosoPol., 1916, Bull. Soc. Imp. Naturalistes Moscou, s.n. 29: 120 .

$\equiv$ Conioselinum ajanense (Regel et Tiling) Khokhr., 1985, Fl. Magadansk. Obl.: 265.

Type: RUSSIA. "Ajan, in sylvis et pascuis [Prope Ajan], Tiling [192]" (lectotype LE!, designated by Pimenov, 1987: 242, as "type"; Vinogradova, 2006: 1285; isolectotypes LE!, P! - barcodes P00602133, P00602134); “Ajan, Tiling” (syntype LE!).

$=$ Conioselinum kamtschaticum Rupr., 1859, Beitr. Pfl. Russ. Reiches 11 (Revis. Umbell. Kamtsch.): 22.

$=$ Conioselinum gmelinii (Cham. et Schltdl.) J. M. Coult. et Rose var. kamtschaticum (Rupr.) Hultén, 1937, Fl. Aleutian Isl.: 248.

Type: RUSSIA. "Kamtschatka, ad Goligyn in planitis arenosa. 1833?, Rieder" (lectotype LE!, designated by Vinogradova, 2006: 1282).

= Cnidium tilingia (Maxim.) Takeda var. pectinatum Koidz., 1917, Bot. Mag. (Tokyo) 31: 128.
= Cnidium ajanense (Regel et Tiling) Drude var. pectinatum (Koidz.) Takeda, 1918, Bot. Mag. (Tokyo) 32: 202.

Type: JAPAN. "In alpibus Nippon mediae et borealis: mt. Chokaisan, 08. 08. 1904, Nakahara" (syntype TI!); "In alpibus Nippon mediae et borealis: Mt. Adsumasan, 15. 08. 1904, Nakahara" (syntype TI!); "In alpibus Nippon mediae et borealis: Mt. Iidesan, Aizu, 10. 08. 1904, Nakahara" (syntype TI!); "In alpibus Nippon mediae et borealis: Nikko, Ontakesan, 08. 1910, Koidzumi” (syntype TI!).

Distribution in China: N: Hebei, Shandong; NE: Heilongjiang, Jilin.

Distribution outside China: Russia, Japan.

Tongoloa cnidiifolia $\mathrm{Fu}$ Kuntsun, 1981, Fl. Tsinling. 1(3): 456, 385, fig. 334.

Type: CHINA. "Shensi: Hwa-yin Hsien, Hwayang Commune, Ta-pai-yang-cha, alt 1760-1990 m, 23. 09. 1975, Fu Kuntsun 17264" (holotype WUK).

Distribution in China: N: Shaanxi. Endemic.

Tongoloa dunnii (H. Boissieu) H. Wolff, 1927, in Engler (ed.), Pflanzenreich 90(IV, 228): 317.

三 Pimpinella dunnii H. Boissieu, 1903, Bull. Herb. Boissier (2 sér.) 3(10): 841.

Type: CHINA. "Prov. Hupeh, Ichang, 03. 1889, Henry 6955" (lectotype P! - barcode P00753108, designated here; isolectotypes E!, GH! - barcode GH0062646, K! - barcode K000695725, LE!, P!).

$=$ Peucedanum giraldii Diels, 1900, Bot. Jahrb. Syst. 29 (3-4) (Fl. Centr. China): 503.

Type: CHINA. "T'ai pa shan bis zur mittleren Höhe [Dalle Jalde alla meta del Thoe-pei-san, Shensi septentrionale (Cina)], 08. 1893, Giraldi 1329" (lectotype FI!, designated here; isolectotype P!).

Distribution in China: Tibet: Xizang A. R.; N: Shanxi, Hebei; NE; Centr.: Hubei; SW: Sichuan. Endemic.

Tongoloa elata H. Wolff, 1926, Acta Horti Gothob. 2: 291.

$\equiv$ Pimpinella elata (H. Wolff) M. Hiroe, 1958, Umbell. Asia 1: 59.

Type: CHINA. "Sze-ch'uan bor.-occid.: Sankar, Talschlucht, auf feuchten Bachwiesen, 4000 m, 03. 09. 1922, H. Smith 4277, 4314" (syntype UPS!); "Prov. Sze-ch'uan, reg. bor.: Huang-ch'en-kuan, in uliginosis herbosis, graminosis ca. $3300 \mathrm{~m}, 19$. 08[05. 09]. 1922, H. Smith 4043" (lectotype UPS!, designated here; isolectotypes GB! - barcode GB0048828, LD! - barcode LD1026390). 
Distribution in China: NW: Gansu; Tibet: Qinghai; SW: Sichuan. Endemic.

Tongoloa filicaudicis $\mathrm{Fu}$ Kuntsun, 1981, F1. Tsinling. 1(3): 456, 385, fig. 335.

Type: CHINA. "Kansu: Wen Hsien, Chung-tsai Commune, Sungping, alt. 2800 m, 16. 07. 1959, C. Y. Chang 6869"' (holotype WUK).

Distribution in China: NW: Gansu. Endemic.

Tongoloa fortunatii (H. Boissieu) Pimenov et Kljuykov, 1999, Feddes Repert. 110(7-8): 489, "furtunatii".

E Pimpinella peucedanifolia H. Boissieu, 1906, Bull. Soc. Bot. France 53: 428, nom. illeg. (Art. 53.1), non Fisch. ex Ledeb. (1844).

三 Pimpinella fortunatii H. Boissieu, 1909, Bull. Soc. Bot. France 56: 351.

$\equiv$ Tongoloa peucedanifolia (H. Boissieu) $\mathrm{H}$. Wolff, 1927, in Engler (ed.), Pflanzenreich 90(IV, 228): 318.

Type: CHINA. "[Yunnan] Loliang (province de Kouy-Tcheou), chute d'eau, 04. 11. 1901, Cavalerie, Fortunat 740" (holotype P! - barcode P00345437).

= Physospermopsis wolffiana Fedde ex H. Wolff, 1929, Repert. Spec. Nov. Regni Veg. 27: 179.

= Pleurospermum cavalerii M. Hiroe, 1979, Umbelliferae World: 746.

Type: CHINA. "Yunnan-sen, district Pin-fa, 1902, Cavalerie 508" (lectotype E! - barcode E00000220, designated here; isolectotypes E barcode E00000221, P!).

= Tongoloa stewardii H. Wolff, 1929, Repert. Spec. Nov. Regni Veg. 27: 185.

= Pimpinella stewardii (H. Wolff) M. Hiroe, 1958, Umbell. Asia 1: 61.

Type: CHINA. "Kiangsi, Kuling, damp ground, Lu Shan, 19. 09. 1922, Steward 2721" (lectotype K! - barcode K000695724, designated here; isolectotype UC).

Distribution in China: Centr.: Anhui, Jiangxi, Zhejiang; SW: Sichuan, Yunnan. Endemic.

Tongoloa gracilis H. Wolff, 1925, Notizbl. Bot. Gart. Berlin-Dahlem 9: 279.

三 Pimpinella tilia M. Hiroe, 1979, Umbelliferae World: 834.

Type: CHINA. "Tibetia orientalis: Tongolo, Soulié 2606" (holotype B: destr.?; isotype BM photo).

Distribution in China: NW: Gansu; Tibet: Xizang A. R., Qinghai; SW: Sichuan, Yunnan.

Distribution outside China: Bhutan.
Tongoloa loloensis (Franch.) H. Wolff, 1927, in Engler (ed.), Pflanzenreich 90(IV, 228): 318.

三Carum loloense Franch., 1894, Bull. Annuel Soc. Philom. Paris (sér. 8) 6: 125.

三 Pimpinella loloensis (Franch.) H. Boissieu, 1902, Bull. Herb. Boissier (2 sér.) 2: 809, in obs.

ETrachydium loloense (Franch.) M. Hiroe, 1958, Umbell. Asia 1: 125.

Type: CHINA. "Province du Yun-nan, in paludosis ad Oua-pin-keou in monte Ma-eul-chan, 23. 10. 1889, Delavay" (syntype P! - barcode P00245440); "Province du Yun-nan: du village Lolo des Pee-tsao-lo (Lakong), 2500 m, 19. 10. 1885, Delavay 2016" (lectotype P! - barcode P00245438, designated here; isolectotypes $\mathrm{A}, \mathrm{GH}$ - barcodes GH00062644, GH00062645, H, NY, P!); "Province du Yun-nan: ad collum Hee-chan-men, 28. 10. 1885, Delavay 2016" (syntypes A - barcode A0083225, GH! - barcode GH00062645, K! - barcode K000075378, NY - barcode NY00405778, P! barcode P00245441).

Distribution in China: Tibet: Xizang A. R.; SW: Sichuan, Yunnan.

Distribution outside China: Bhutan, Nepal, India.

Tongoloa pauciradiata H. Wolff, 1929, Repert. Spec. Nov. Regni Veg. 27: 128.

$\equiv$ Pimpinella pauciradiata (H. Wolff) M. Hiroe, 1979, Umbelliferae World: 833.

Type: CHINA. "Tibet, Lhasa, ca. 12000 feet s.1., 30. 08. 1904, Waddell" (holotype K! - barcode K000075377).

Distribution in Asia: Tibet: Xizang A. R., Qinghai. Endemic.

Tongoloa rockii H. Wolff, 1929, Repert. Spec. Nov. Regni Veg. 27: 127.

$\equiv$ Pimpinella yangtzensis M. Hiroe, 1979, Umbelliferae World: 833, "yaugtzensis".

Type: CHINA. "Yunnan: between Likiang, Tungshan, Tuinaoko and Tsilikiang, dry Yangtze drainage basin, 13-14000' s.1., 05. 1923, Rock 9758" (lectotype E!, designated here; isolectotypes GH! - barcode GH00077982, US).

Distribution in China: SW: Yunnan, Endemic.

Tongoloa rubronervis Liou Shoulu, 1989, Acta Phytotax. Sin. 27(1): 69, tab. 1, fig. 6-11.

Type: CHINA. "Sichuan, Muli, alt. 3670 m, Pu Fating 162" (holotype NAS).

Distribution in China: SW: Sichuan, Yunnan. Endemic. 
Tongoloa silaifolia (H. Boissieu) H. Wolff, 1925, Notizbl. Bot. Gart. Berlin-Dahlem 9: 280.

$\equiv$ Pimpinella silaifolia H. Boissieu, 1902, Bull. Herb. Boissier (2 sér.) 2: 809.

Type: CHINA. "Su-Tchuen a Tchen-Kéou-Tin [pelouses a Siao Kieou teou tcha, dans la souspréfecture de Talin hieh, alt. $2200 \mathrm{~m}$ ], 26. 08. 1898, Farges 1427" (lectotype P! - barcode P00245442, designated here; isolectotypes $\mathrm{B}$ !, K, P! - barcodes P00245443, P00245444, P022884794, P022884795, P022884796, PE!).

Distribution in China: Tibet: Xizang A. R., Qinghai; N: Ningxia Huizu A. R., Shaanxi; Centr.: Hubei; SW: Sichuan, Yunnan. Endemic.

Tongoloa smithii H. Wolff, 1926, Acta Horti Gothob. 2: 290.

Type: CHINA. "Sze-ch'uan bor.-occid.: Sankar vou ma, auf Mooren, 4000 m, 05. 09. 1922, H. Smith 4270" (lectotype UPS!, designated here; isolectotypes BM (photo), GB! - barcode GB0048829).

Distribution in China: SW: Sichuan. Endemic.

Tongoloa taeniophylla (H. Boissieu) H. Wolff, 1925, Notizbl. Bot. Gart. Berlin-Dahlem 9: 280.

$\equiv$ Pimpinella taeniophylla H. Boissieu, 1906, Bull. Soc. Bot. France 53: 429.

Type: CHINA. "Ost-Tibet: Ta-Tsien-Lou, 07. 1894, Soulié 2174" (lectotype P! - barcode P00245446, designated here).

= Acronema chienii Shan Renhwa, 1980, Acta Phytotax. Sin. 18(2): 197, tab. 1.

Type: CHINA. "Sichuan, Danba, K. L. Chu 7705" (holotype NAS).

= Acronema chienii Shan Renhwa var. dissectum Shan Renhwa, 1980, Acta Phytotax. Sin. 18(2): 198.

Type: CHINA. "Sichuan, Danba, K. L. Chu 7535" (holotype NAS).

= Changium angustilobum P. K. Mukh. et Kljuykov, 1991, Byull. Moskovsk. Obšč. Isp. Prir. Otd. Biol. 96, 5: 71.

Type: CHINA. "Sikang: Taofu (Dawo), distr. Taining (Ngata). Inter Tjedji et Maioniu in silva muscosa, ca. 3000 m s.l., 30. 09. 1934, H. Smith 12601" (lectotype UPS!, designated in protologue as "typus"; isolectotype GB!).

Distribution in China: Tibet: Xizang A. R., Qinghai; SW: Sichuan, Yunnan. Endemic.

Tongoloa tenuifolia $\mathrm{H}$. Wolff, 1929, Repert. Spec. Nov. Regni Veg. 27: 128. $\equiv$ Pimpinella likiangensis M. Hiroe, 1979, Umbelliferae World: 833.

Type: CHINA. "Yunnan, Yangtze watershed. Prefectural district of Likiang, eastern slopes of Likiang Snow Range, in swamps, 16. 08. 1922, Rock 5853" (holotype ?; isotype US).

Distribution in China: Tibet: Xizang A. R.; SW: Sichuan, Yunnan. Endemic.

Tongoloa zhongdianensis Liou Shoulu, 1989, Acta Phytotax. Sin. 27(1): 68, tab. 1, fig. 1-5.

Type: CHINA. "Yunnan, Zhongdian, alt. 2800 m, 18 .09. 1962, Exped. Zhongdian 2149" (holotype KUN!),

Distribution in China: SW: Yunnan. Endemic.

Tordyliopsis brunonis DC., 1830, Prodr. 4: 199. $\equiv$ Heracleum brunonis (DC.) C. B. Clarke, 1879, in Hooker f. (ed.), Fl. Brit. India 2: 713..

Type: INDIA. "In Kamaon Nepaliae versus Emodum, Blinkworth [590]" (lectotype G-DC, designated by Watson, 1998:383; isolectotypes BM!barcodes BM000944789, BM000944790, CAL, CGE!, E! - barcode E00002937, K, K-WALLICH! barcodes K000075516, K000075517, P).

Distribution in China: Tibet: Xizang A. R.

Distribution outside China: Bhutan, Nepal, India.

Torilis japonica (Houtt.) DC., 1830, Prodr. 4: 219.

$\equiv$ Caucalis japonica Houtt., 1777, Handl. P1.Kruidk. 8: 42, tab. 45, fig. 1.

$\equiv$ Torilis anthriscus (L.) C.C.Gmelin var. japonica (Houtt.) H. Boissieu, 1903, Bull. Herb. Boissier (2 sér.) 3(10): 856.

Type: JAPAN. "Houttuyn" (lectotype G-Herb. Burman; designated by Burdet, Jacquemoud, Reduron in Reduron, 2008: 2524).

$=$ Tordylium anthriscus L., 1753, Sp. pl.: 240.

= Caucalis anthriscus (L.) Huds., 1762, Fl. Angl.: 99.

= Anthriscus vulgaris Bernh., 1800, Syst. Verz.: 168.

Type: EUROPE (lectotype BM-Cliff 90, Tordylium 5; designated by Jury, Southam and Reduron in Jarvis et al., 2006: 215).

$=$ Caucalis aspera Lam., 1779, Fl. Franç. 3: 424.

= Torilis stricta Wibel, 1799, Prim. fl. Werth.: 192.

= Torilis rubella Moench, 1794, Methodus: 103.

= Tordylium verecundum Salisb., 1796, Prodr. Stirp. Chap. Allerton: 160. 
$=$ Torilis anthriscus (L.) C.C.Gmelin, 1805, Fl.

Bad. 1: 168, nom. illeg. (Art. 53.1), non Gaertn. (1788).

= Daucus anthriscus (L.) Baill., 1879, Hist. Pl. 7: 88.

$=$ Selinum torilis E. H. L. Krause, 1904, in Sturm (ed.), Fl. Deutschl. (ed. 2) 12: 155.

$=$ Chaerefolium anthriscus (L.) Schinz et Thell., 1908, Vierteljahrsschr. Naturf. Ges. Zürich 53: 554.

Type: N EUROPE. "In Europae septentrionalis arvis ruderatis, Herb. Clifford 90, Tordylium 5" (lectotype BM-Cliff, designated by Jury, Southam, Reduron in Jarvis et al., 2006: 215).

$=$ Caucalis elata D. Don, 1825, Prodr. Fl. Nepal.: 183.

$=$ Torilis elata (D. Don) Spreng., 1827, Syst. Veg. (ed. 16) 4 (2, Cur. Post.): 119; ex DC., 1830, Prodr. 4: 220.

Type: NEPAL. "M. altiss. Nepaliae, 1821, Buchanan-Hamilton 565" (lectotype K-WALLICH!, designated here; isolectotype CAL, M).

= Caucalis orientalis Lour., 1790, Fl. Cochinch.: 177, nom. illeg. (Art. 53.1), non L. (1753).

Type: CHINA. "Hab. in culta Cantone et alibi".

$=$ Torilis persica Boiss. et Buhse, 1860, Nouv. Mém. Soc. Imp. Naturalistes Moscou 12: 102.

Type: AZERBAIJAN. "Achewlar im Talyschgebirge, 29. 08. 1847, Buhse" (holotype LE!).

= Torilis praetermissa Hance, 1866, Ann. Sci. Nat. Bot. (sér. 5) 5: 214.

Type: CHINA. "Ad margines oryzetorum atque in incultis circa Cantonem, 06.1865, Hance (Sampson) 11399" (lectotype K - barcode K000695518; syntypes BM - barcode BM000944707, K!, NY).

Distribution in China: NW: Xinjiang Weiwuer A. R., Gansu; Tibet: Xizang A. R., Qinghai; N: Neimenggu A. R., Ningxia Huizu A. R., Shaanxi, Shanxi, Hebei, Shandong; NE: Jilin, Liaoning; Centr.: Henan, Jiangsu, Anhui, Hubei, Hunan, Jiangxi, Zhejiang, Fujiang; SW: Sichuan, Guizhou, Yunnan; S: Taiwan, Guangxi Zhuang A. R., Guangdong.

Distribution outside China: Russia, Japan, Korea, Indonesia, Malaysia, Vietnam, Myanmar, Bhutan, Nepal, India, Pakistan, Afghanistan, Iran, Azerbaijan, Georgia, Armenia, Turkey, Syria, Jordan. Europe, Africa, N. America, Australia and Oceania.

Torilis scabra (Thunb.) DC., 1830, Prodr. 4: 219. EChaerophyllum scabrum Thunb. ex A.Murray (ed.), 1784, Syst. Veg. (ed. 14): 289; Thunb., 1784, Fl. Jap.: 119. $\equiv$ Myrrhis scabra (Thunb.) Spreng., 1824, Syst. Veg. (ed. 16) 1: 902.

三 Caucalis scabra (Thunb.) Makino, 1893, Bot. Mag. (Tokyo) 7: 44.

三Anthriscus scabra (Thunb.) Koso-Pol., 1916, Bull. Soc. Imp. Naturalistes Moscou, s.n. 29: 151.

Type: JAPAN. "Crescit prope Iedo [Tokyo] et alibi" (UPS?).

= Caucalis praetermissa Hance ex Franch., 1879, Bull. Soc. Bot. France 26: 86.

Type: JAPAN. "Insula Nippon. Vidi e viciniis Yedo, Yosioka, Niko, Niigata, Savatier, Faurie, Dickins" (syntypes).

$=$ Torilis henryi C. Norman, 1929, J. Bot. 67: 147.

Type: CHINA. "Hupeh, 1885-1888, Henry 1349" (lectotype BM! - barcode BM000944708, designated here; isolectotypes $\mathrm{K}$ ! - barcode K000695516, US).

Distribution in China: NW: Gansu; N: Ningxia Huizu A. R., Shaanxi, Shandong; Centr.: Henan, Jiangsu, Anhui, Hubei, Hunan, Jiangxi, Zhejiang, Fujiang; SW: Sichuan, Guizhou; S: Taiwan, Guangxi Zhuang A. R., Guangdong.

Distribution outside China: Japan, Korea. N. America.

Trachydium involucellatum Shan Renhwa et $\mathrm{Pu}$ Fating, 1986, Acta Phytotax. Sin. 24(4): 313.

Type: CHINA. "Xizang: Zayu, alt. 4000-4500 m, 26. 09. 1982, Exped. Qinghai-Xizang 10626" (holotype PE; isotype KUN!).

Distribution in China: Tibet: Xizang A. R.; SW: Yunnan. Endemic.

Trachydium napiferum H. Wolff, 1926, Acta Horti Gothob. 2: 300.

$\equiv$ Tongoloa napifera (H. Wolff) C. Norman, 1938, J. Bot. 76: 232.

Type: CHINA. "Sze-ch'uan bor.: San-ch'a-tzü, auf etwas erdigen, krautigen Wiesen, 4000 m, 10. 08. 1922, H. Smith 3222, 3204" (lectotype UPS!, designated here; isolectotypes GB! - barcode GB0048830, PE!, W!).

Distribution in China: SW: Sichuan. Endemic.

Trachydium roylei Lindl., 1835, in Royle (ed.), Ill. Bot. Himal. Mts.: 232.

三 Aulacospermum roylei (Lindl.) Kachroo, U. Dhar et Naqshi, in P. Kachroo (ed.), 1995, Central Asia \& Kashmir Himalaya: 107, nom. inval., sine basion.

Type: INDIA. "Peer Punjal Pass, Royle" (lectotype CGE!, designated here; isolectotype K!). 
Distribution in China: Tibet: Xizang A. R.

Distribution outside China: India, Pakistan.

Trachydium simplicifolium W. W. Sm., 1915, Notes Roy. Bot. Gard. Edinburgh 8: 346.

$\equiv$ Ligusticum simplicifolium (W. W. Sm.) M. Hiroe, 1958, Umbell. Asia 1: 108.

Type: CHINA. "Yunnan, West China: eastern flank of the Lichiang Range. Lat. 27'30' N. Alt. 9-10000 ft, 10. 1910, Forrest 7394" (holotype E! barcode E00000295).

Distribution in China: Tibet: Xizang A. R.; SW: Yunnan. Endemic.

Trachydium souliei H. Boissieu, 1906, Bull. Soc. Bot. France 53: 422.

Type: CHINA. "Thibet oriental: Tsekou, montagne de Tsen-Tchiong, 20. 09. 1893, Soulié 1049" (lectotype P! - barcode P00245463, designated here; isolectotype P! - barcode P00834666).

= Trachydium tibetanicum H. Wolff, 1929, Repert. Spec. Nov. Regni Veg. 27: 122.

Type: CHINA. "SE Tibet: Salwin-Kin-Shang divide, $28^{\circ} 40^{\prime} \mathrm{N}, 98^{\circ} 15^{\prime} \mathrm{E}$, in open alpine pasture, 07. 1919, Forrest 19061" (lectotype E!, designated here; isolectotypes BM! - barcode BM000849645, K - barcode K001091311, P!, W!).

= Sinocarum woodii M. F. Watson, 1996, Edinburgh J. Bot. 53(1): 143, fig. 4, D-F.

Type: BHUTAN. "Thimphu district, near Nya Tsho, Saga La, 4400 m, 25 .08. 1991, Wood 7440” (holotype E - barcode E00041756).

Distribution in China: Tibet: Xizang A. R., Qinghai; SW: Sichuan, Yunnan.

Distribution outside China: Bhutan, Nepal, India.

Trachydium subnudum C. B. Clarke ex H. Wolff, 1929, Repert. Spec. Nov. Regni Veg. 27: 125.

$\equiv$ Chamaesciadium subnudum (C. B. Clarke ex H. Wolff) C. Norman, 1938, J. Bot. 76: 233.

Type: CHINA. "Tibet, Khambajong, 09. 1903, Prain" (lectotype K! - barcode K000695715, designated by Watson, 1998: 372; isolectotypes BM! - barcode BM000885952, CAL, E! - barcode E00000465, K!, P! - barcode P00834622); "Tibet, Khambajong, 07.09.1903, Younghusband 310" (syntype K! - barcode K000695714).

$=$ Trachydium verrucosum Shan Renhwa et $\mathrm{Pu}$ Fating, 1979, in Shan Renhwa \& Sheh Menglan (eds.), Fl. Reipubl. Popularis Sin. 55(1): 299, 203, tab. 108, fig. 1-8.

Type: CHINA. "Tibet, La-sa, Nai-go-ma-Shan, alt. 5000 m, 18. 08. 1965, Y. T. Chang, K. Y. Lang 1885 " (holotype PE; isotype KUN!).
$=$ Trachydium lamondianum Farille et S. B. Malla, 1985, Candollea 40(2): 533, tab. 5, fig. 7 (1-3).

Type: INDIA. "East Himalaya: Phari, 14000 feet, 12. 09. 1912, Rohmoo Lepcha 252" (holotype E! - barcode E00000469); isotypes A - barcode A00077984, G! - barcode G00367357).

Distribution in China: Tibet: Xizang A. R.; SW: Sichuan.

Distribution outside China: Nepal.

Trachydium szechuanense H. Wolff, 1926, Acta Horti Gothob. 2: 299.

Type: CHINA. "Sze-ch'uan bor.: San-ch'a-tzu, auf alpinen Wiesen. Kalk, 4000 m, 10. 08. 1922, H. Smith 3428" (holotype UPS!).

Distribution in China: SW: Sichuan. Endemic.

Trachydium trifoliatum $\mathrm{H}$. Wolff, 1929, Repert. Spec. Nov. Regni Veg. 27: 125.

Type: CHINA. "W. Yunnan: Shmeli-Salwin divide. Lat. $25^{\circ} 45^{\prime}$ N. Long. $98^{\circ} 58^{\prime}$ ' E. Alt. $12000 \mathrm{ft}$. On stony alpine meadows, 10. 1924, Forrest 25265" (holotype E! - barcode E00000298).

Distribution in China: Tibet: Xizang A. R.; SW: Yunnan. Endemic.

Trachydium variabile $\mathrm{H}$. Wolff, 1926, Acta Horti Gothob. 2: 298.

Type: CHINA. "Sze-ch'uan bor:: San-ch'a-tzu, auf Kalk, Alpenwiesen, 4000 m, 10. 08. 1922, H. Smith 3205" (lectotype UPS!, designated here; isolectotype W!); "Sze-ch'uan bor.: Dongrergo, oberhalb Huang-lung-ssu, ca. 4500 m, 21. 08. 1922, H. Smith 3232,3723, 3888" (syntypes GB! - barcode GB-0048832, PE!, UPS!); “Sze-ch'uan bor--occid.: Tsipula, auf Alpenwiesen, ca. 4000 m, 08. 1922, H.Smith 2988,4181" (syntypes BM! - barcode BM000826941, GB! - barcode GB-0048831, UPS!, $\mathrm{W} !)$.

= Trachydium dielsianum $\mathrm{H}$. Wolff, 1926, Acta Horti Gothob. 2: 300.

Type: CHINA. "Sze-ch'uan bor--occid.: südostlich von Matang, Alpenwiesen, $4800 \mathrm{~m}$ [Mont. - austro-orient., in alpinetis] 13. 09. 1922, H. Smith 4375" (holotype UPS!).

Distribution in China: SW: Sichuan, Yunnan. Endemic.

Trachydium wolffianum Fedde ex H. Wolff, 1929, Repert. Spec. Nov. Regni Veg. 27: 122.

Type: CHINA. "Tibet: A-tun-tsi, turf, 14000 feet, 13. 08. 1913, Kingdon-Ward 799a".

Distribution in China: SW: Yunnan. Endemic. 
Trachyspermum roxburghianum (DC.) H. Wolff, 1927, in Engler (ed.), Pflanzenreich 90(IV, 228): 129.

E Ptychotis roxburghiana DC., 1830, Prodr. 4: 109.

ECarum roxburghianum (DC.) Waring, 1868, Pharmacop. India: 108, nom. illeg. (see: Turner, 2016).

Type: SINGAPORE. "In insula Singaporia (Singapore), cult., Wallich [571]" (lectotype G-DC, designated by R. K. Singh, 2015: 248, fig. 3; isolectotypes K!, K-WALLICH!, LE!). INDIA. "India orientalis, Heyne" (syntype G-DC). MYANMAR. "Montes Prome, 1827, Wallich" (syntype G-DC); "Prome, 1829, Wallich 571" (syntupes CAL, K).

= Apium involucratum Roxb. ex Fleming (ed.), 1810, Asiat. Res. 11: 157.

= Pimpinella involucrata (Roxb. ex Flem.) Wight et Arn., 1834, Prodr. F1. Penins. Ind. Orient. 1: 369.

= Ptychotis involucrata (Roxb. ex Flem.) Lindl., 1835, in Royle, Ill. Bot. Himal. Mts.: 229.

= Carum involucratum (Roxb. ex Flem.) Baill. ex Kuntze, 1891, Revis. Gen. P1. 1: 265.

= Trachyspermum involucratum (Roxb. ex Flem.) H. Wolff, 1927, in Engler (ed.), Pflanzenreich 90(IV, 228): 89, nom. illeg. (Art. 53.1), non Maire (1926).

EFalcaria diversifolia DC., 1830, Prodr. 4: 110 .

= Carum diversifolium (DC.) C. B. Clarke, 1879 , in Hooker f. (ed.), Fl. Brit. India 2: 681.

Type: NEPAL. "Nepalia, 1821, Wallich [592]" (holotype K-WALLICH!; isotype CAL).

= Carum involucratum (Roxb. ex Flem.) Baill. ex Kuntze var. anomalum Kuntze, 1891, Revis. Gen. P1. 1: 269.

= Trachyspermum involucratum (Roxb. ex Flem.) H. Wolff var. anomalum (Kuntze) H. Wolff, 1927, in Engler (ed.), Pflanzenreich 90(IV, 228): 90.

Type: INDIA. "West Ghats, Kuntze".

Distribution in China: SW: Yunnan.

Distribution outside China: Philippines, Papua-New Guinea, Indonesia, Malaysia, Vietnam, Laos, Cambodia, Thailand, Myanmar, Nepal, India, Sri-Lanka.

Turgenia latifolia (L.) Hoffm., 1814, Gen. P1. Umbell.: 59.

三Tordylium latifolium L., 1753, Sp. Pl.: 240.

$\equiv$ Caucalis latifolia (L.) L., 1767, Syst. Nat. (ed. 12) $2: 205$.

三Daucus latifolius (L.) Baill., 1879, Hist. P1. 7: 193, "latifolia". $\equiv$ Daucus turgenia E. H. L. Krause, 1904, in Sturm (ed.), Deutschl. Fl. (ed. 2) 12: 158, nom. illeg. (Art. 52.1).

Type: EUROPE. "Gallia et Italia 338.7" (lectotype LINN!, designated by Reduron, Jarvis in Jarvis et al., 2006: 210; see also Reduron, 2008: 2580).

= Turgenia multiflora DC., 1830, Prodr. 4: 218.

= Turgenia latifolia (L.) Hoffm. var. multiflora (DC.) Boiss., 1872, Fl. Orient. 2: 1087.

Type: TURKEY. "In agro Bysantino, Prescott" (holotype G-DC!).

= Turgenia brachyacantha Boiss., 1844, Ann.

Sci. Nat. Paris (sér. 3, Bot.) 2 (P1. Aucher.): 52.

= Turgenia latifolia (L.) Hoffm. var. brachyacantha (Boiss.) Boiss., 1872, F1. Orient. 2: 1087.

Type: IRAN. "In Persia australi, AucherEloy[4563]" (lectotype P!, designated here; isolectotype G-BOIS!).

= Turgenia tuberculata Boiss., 1844, Ann. Sci. Nat. Paris (sér. 3, Bot.) 2 (P1. Aucher.): 52.

= Turgenia latifolia (L.) Hoffm. var. tuberculata (Boiss.) Boiss., 1872, Fl. Orient. 2: 1087.

Type: SYRIA. "In Syriâ, Aucher-Eloy 3639" (lectotype G-BOIS!, designated here by Pimenov, Jacquemoud; isolectotypes LE!, MPU, P); "In agris prope Aleppum, 07. 05. 1841, Kotschy 191" (syntypes G-BOIS!, P).

Distribution in China: NW: Xinjiang Weiwuer A. R.

Distribution outside China: Russia, India, Pakistan, Kazakhstan, Kirghizia, Tajikistan, Uzbekistan, Turkmenistan, Afghanistan, Iran, Azerbaijan, Georgia, Armenia, Turkey, Iraq, Lebanon, Syria, Israel, Jordan, Cyprus. Europe, Africa, N. America.

Vicatia atrosanguinea (Kar. et Kir.) P. K. Mukh. et Pimenov, 1991, Feddes Repert. 102(5-6): 377, fig. 1.

$\equiv$ Carum atrosanguineum Kar. et Kir., 1842, Bull. Soc. Imp. Naturalistes Moscou 15: 359.

Type: KAZAKHSTAN. "In umbrosis muscosis rupium summarum alpium Alatau ad fl. Lepsa et Sarchan, 1841, Karelin, Kirilov 1510" (lectotype LE!, designated by Vinogradova, 1999: 84; isolectotypes MW!, TK).

= Pimpinella saxifraga L. var. dissectifolia $\mathrm{C}$. B. Clarke, 1879, in Hooker f. (ed.), F1. Brit. India 2: 685, nom illeg. (Art. 53.1), non Boiss. (1872).

Type: INDIA. 'Kashmir, Tilail, alt. 13000', Clarke" (syntype K?). PAKISTAN. "Karakorum, alt. 14000', Clarke"' (syntype K?). 
= Tongoloa wolffiana Fedde ex H. Wolff, 1929, Repert. Spec. Nov. Regni Veg. 27: 181.

= Vicatia wolffiana (Fedde ex H. Wolff) C. Norman, 1940, J. Bot. 78: 229.

Type: INDIA. "N.W.Himalaya, Tihri-Garhwal, Ourie Gadh, in Nila valley, 14-15000', 15. 08. 1883. Duthie 1134" (lectotype K!, designated by Pimenov in Pimenov, Ostroumova, 2012: 149; isolectotypes BM, CAL, DD!, G! - barcode G00367407); “Alampi-la, 14000', Duthie 12176" (syntypes CAL, DD).

Distribution in China: NW: Xinjiang Weiwuer A. R.

Distribution outside China: Russia, India, Pakistan, Kazakhstan, Kirghizia, Tajikistan, Afghanistan.

Vicatia coniifolia DC., 1830, Prodr. 4: 243.

$\equiv$ Sison coniifolium Wall. ex DC., 1830, Prodr. 4: 243.

$\equiv$ Conium nepalense D.Dietr., 1840, Syn. Pl. 2: 988.

$\equiv$ Sphallerocarpus coniifolius (DC.) Koso-Pol., 1916, Bull. Soc. Imp. Naturalistes Moscou, s.n. 29: 202.

Type: INDIA. "In Nepalia ad Kamaon, 1829, Wallich [591.2]" (lectotype G-DC!, designated by Watson, 1998: 383; isolectotypes CAL, E!, K!, K-WALLICH!, LE!, PH).

= Chaerophyllum millefolium Klotzsch, 1862, in Klotzsch \& Garcke (eds.), Bot. Ergebn. Reise Waldemar: 149, tab. 45, nom. illeg., non DC. (1830).

= Vicatia millefolia (Klotzsch) C. B. Clarke, 1879, in Hooker f., (ed.) Fl. Brit. India 2: 671.

= Sphallerocarpus millefolius (Klotzsch) KosoPol., 1916, Bull. Soc. Imp. Naturalistes Moscou, s.n. 29: 202.

Type: INDIA. "Im Himalaya, 1845, Hoffmeister".

= Chaerophyllum gracillimum Klotzsch, 1862, in Klotzsch \& Garcke (eds.), Bot. Ergebn. Reise Waldemar: 149, tab. 46.

Type: INDIA. "Im Himalaya, 1845, Hoffmeister".

=Vicatia stewartii C. B. Clarke, 1879, in Hooker f. (ed.), Fl. Brit. India 2: 671.

Type: INDIA. "Gurhwal, alt. 6500', Stewart 888" (holotype K!).

$=$ Chaerophyllum nuristanicum Rech. f. et Riedl, 1963, K. Danske Vid.Selsk., Biol. Skrift. 13(4) (Symb. Afghan. 5): 44, fig. 16.

Type: AFGHANISTAN. "NE Afghanistan: Nuristan: Derin-Elasoon, 06. 07. 1949, Edelberg 1716" (holotype C!; photo W).
Distribution in China: Tibet: Xizang A. R., Qinghai; SW: Sichuan, Yunnan.

Distribution outside China: Bhutan, Nepal, India, Pakistan, Afghanistan.

Zosima korovinii Pimenov, 1976, Byull. Glavn. Bot. Sada (Moscow) 101: 45.

Type: KIRGHIZIA. "Asia Media, jugum Kirghisicum, pars orientalis, vallis fluvii Tschu, angustis Boam, 27. 05. 1970, Pimenov 11" (holotype LE!; isotypes B!, GB!).

- Zosima tordyloides auct. non Korovin: Schischk., 1951, in Schischkin, Fl. URSS 17: 267.

Distribution in China: NW: Xinjiang Weiwuer A. R.

Distribution outside China: Kazakhstan, Kirghizia, Tajikistan, Uzbekistan.

\section{Discussion}

As a result, up to the end of 2016 China boasts of the highest species diversity in the Umbelliferae (657 spp, belonging to 110 genera), among Asian countries, and the most probably in the whole world. At the same time Chinese Umbelliferae seem to cause many taxonomical problems, besides comparatively recent publication of some general treatments of the family - in Chinese (Shan Renhwa, Sheh Menglan (eds.), 1979-1992; Fu LiKuo et al. (ed.), 2003) and in English (Sheh MengLan et al., 2005), as well as numerous treatments in provincial Floras, some being original in taxonomy, and all containing regional phytogeographical data. Numerous Chinese Umbelliferae species remain rather enigmatic; some of them have been collected only once. For instance, H. Wolff in the 1920s described scores of species from China, then their type materials were lost during World War II, and now their interpretation is sometimes arbitrary. Wolff's species, published posthumously, seem especially questionable. Earlier French collections, kept in perfect order in Paris (P), and containing numerous type sheets of SW Chinese species, described by A. Franchet and H. de Boissieu, were not properly used during compilation of "Flora Reipublicae Popularis Sinicae". As a result, some species, described by Chinese botanists, were put in synonymy. At the same time, it is obvious, that in future China will come out with quite a few new species.

The above number of species and genera concerns with the Umbelliferae in traditional (Drude's) interpretation of the family volume, adopted earlier in "Flora Reipublicae Popularis Sinicae". Then the most significant change was rejection of the subfamily Hydrocotyloideae (Plunkett et al., 2004; 


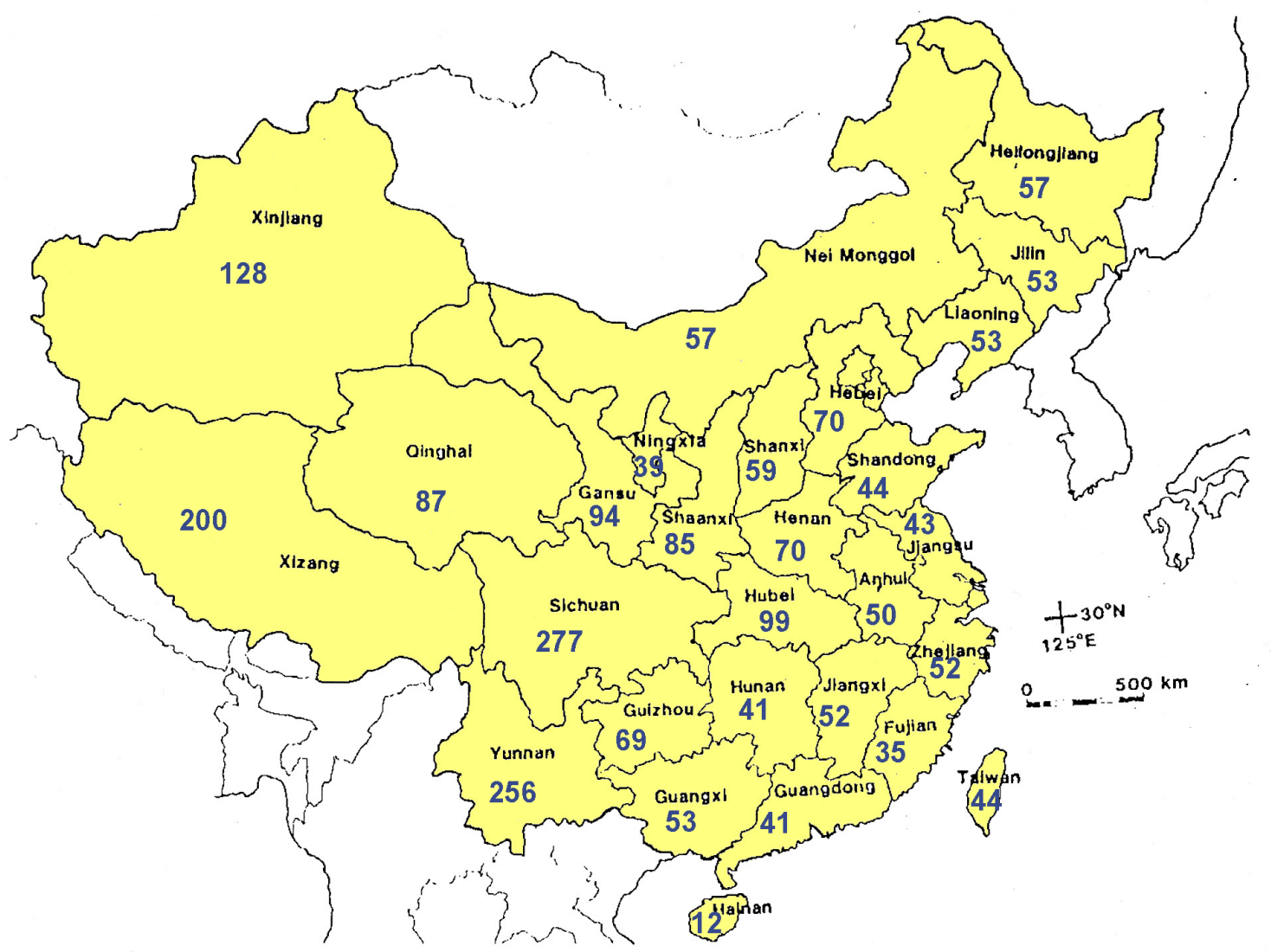

Fig. 1. Numbers of the Umbelliferae species in provinces and autonomous regions of China.

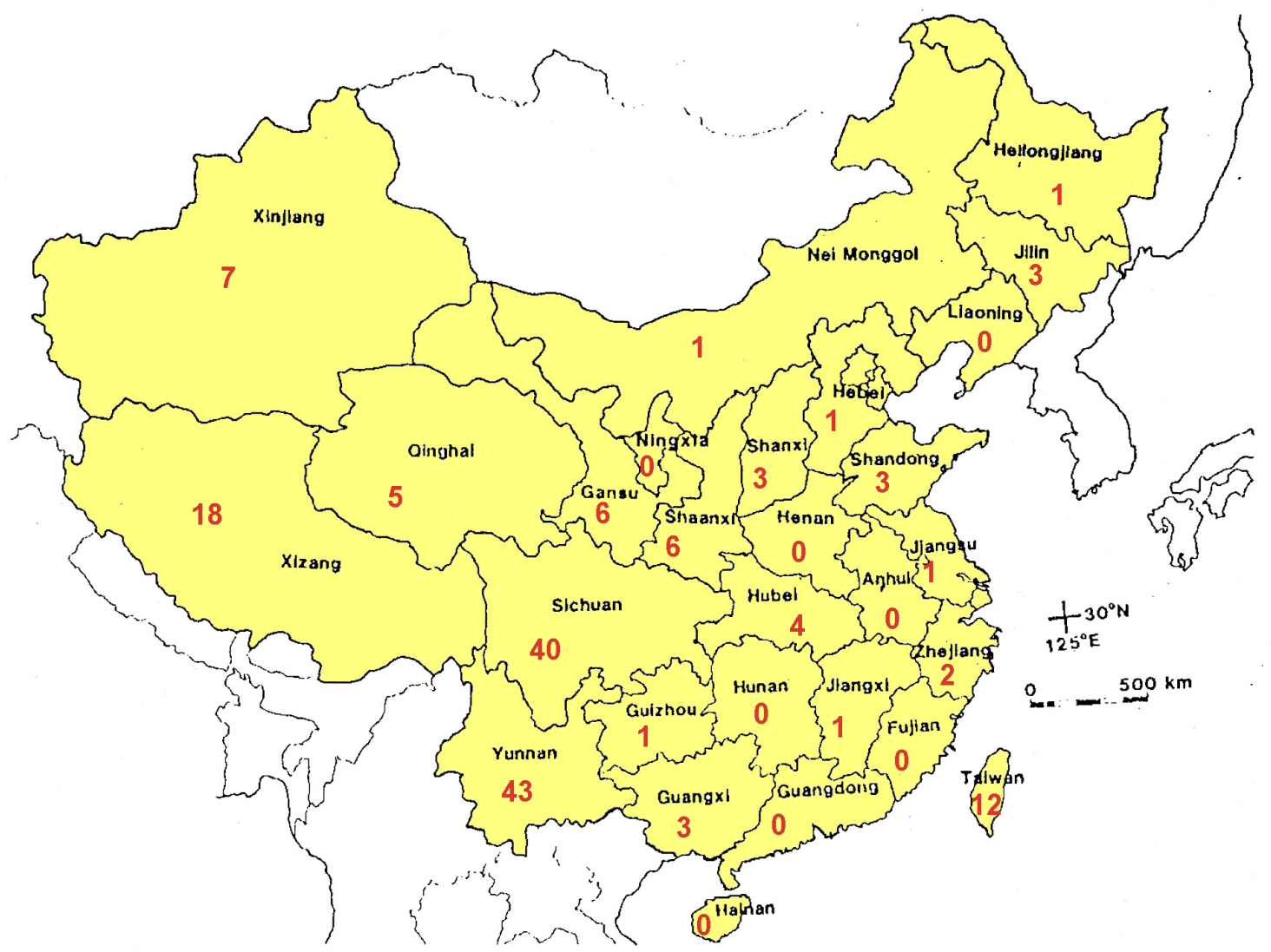

Fig. 2. Numbers of endemic species of the Umbelliferae in provinces and autonomous regions of China. 
Table

Distribution of the Umbelliferae genera, species and endemic species in China

\begin{tabular}{|c|c|c|c|c|}
\hline Country's part & Province or A. R. & Genera & Species & Endemic species \\
\hline \multirow[t]{3}{*}{ NW China } & & 69 & 208 & 13 \\
\hline & Xinjiang A.R. & 57 & 128 & 7 \\
\hline & Gansu & 35 & 94 & 6 \\
\hline \multirow[t]{3}{*}{ Tibet } & & 60 & 233 & 26 \\
\hline & Xizang A. R. & 55 & 200 & 18 \\
\hline & Qinghai & 37 & 87 & 5 \\
\hline \multirow[t]{7}{*}{ N China } & & 46 & 138 & 14 \\
\hline & Neimenggu A. R. & 32 & 57 & 1 \\
\hline & Ningxia Huizu A. R. & 23 & 39 & 0 \\
\hline & Shaanxi & 35 & 85 & 6 \\
\hline & Shanxi & 36 & 59 & 3 \\
\hline & Hebei & 38 & 70 & 1 \\
\hline & Shandong & 28 & 44 & 3 \\
\hline \multirow[t]{4}{*}{ NE China } & & 35 & 78 & 6 \\
\hline & Heilongjiang & 27 & 57 & 1 \\
\hline & Jilin & 25 & 53 & 3 \\
\hline & Liaoning & 30 & 53 & 0 \\
\hline \multirow[t]{9}{*}{ Central China } & & 49 & 148 & 13 \\
\hline & Henan & 36 & 70 & 0 \\
\hline & Jiangsu & 31 & 43 & 1 \\
\hline & Anhui & 32 & 50 & 0 \\
\hline & Hubei & 38 & 99 & 4 \\
\hline & Hunan & 24 & 41 & 0 \\
\hline & Jiangxi & 27 & 52 & 1 \\
\hline & Zhejiang & 28 & 52 & 2 \\
\hline & Fujiang & 20 & 35 & 0 \\
\hline \multirow[t]{4}{*}{ SW China } & & 62 & 357 & 126 \\
\hline & Sichuan & 57 & 277 & 40 \\
\hline & Guizhou & 31 & 69 & 1 \\
\hline & Yunnan & 57 & 256 & 43 \\
\hline \multirow[t]{5}{*}{ S China } & & 29 & 80 & 15 \\
\hline & Taiwan & 20 & 44 & 12 \\
\hline & Guangxi Zhuang A. R. & 24 & 53 & 3 \\
\hline & Guangdong & 22 & 41 & 0 \\
\hline & Hainan Dao & 7 & 12 & 0 \\
\hline
\end{tabular}

Nicolas, Plunkett, 2009) as a taxon unrelated to the rest of true Umbelliferae. This novelty, based on new molecular data, is reasonable, being partly supported by morphological data (nodal anatomy and chromosome numbers). The majority of former Hydrocotyloideae genera were, however, included into one of two new subfamilies of Umbelliferae, namely Azorelloideae.The majority was transferred to Azorelloideae, but not Hydrocotyle, a type genus of Hydrocotyloideae, which was put to Araliaceae. It is unreasonable in term of morphology, as the characteristics of Araliaceae must be considerably changed in fundamental structural characters. I believe the question requires further elaboration, and now I would prefer to be conservative.
As to Chinese Umbelliferae, the rejection of traditional Hydrocotyloideae boils down to exclusion from them only genus Hydrocotype, numbering in China 20 species. The rest will be 635 species of the family.

The largest Umbelliferae genera in Chinese flora are Angelica (49 species), Bupleurum (47), Pimpinella (46), Seseli (39), Hymenidium (37), Heracleum (31), Ferula (27), Pternopetalum (21), and Acronema (21). The polymorphic and obviously polyphyletic genus Peucedanum remains to be the most critical for Chinese Umbelliferae, as well as for many countries of temperate North hemisphere, besides some progress with splitting some of its segregates (Kitagawia, Ferulopsis, Haloselinum). 
Umbelliferae are unevenly distributed in China (Tabl.) Many provinces of North, North-East and Central China are poor, at least as compared with neighboring countries; endemic species being not numerous either. High diversity in Chinese Umbelliferae is due to numerous species, growing in SW China, especially in Sichuan and Yunnan, in the adjacent part of Xizang A. R., and to a less extent in Xinjiang A. R. We have registered 357 species, including 126 endemics, in SW China, 277 with 40 endemics in the province of Sichuan, and 256 with 43 endemics in the province of Yunnan. Within these two provinces the maximal diversity is observed in the W (Sichuan) and NW (Yunnan) mountain parts on the border of Tibetan plateau (former Sikan, which included the nearby territory, now being Xizang A. R. also rich in Umbelliferae). The tropical South of China is poor in the Umbelliferae, although there are some (12) endemic species in Taiwan. Among Central-Chinese provinces only Hubei with 99 species, 4 of which are endemic, is of interest, whereas in North China Shaanxi with 85 species and 6 endemics is such province. Geographically Hubei and Shaanxi are adjacent to Sichuan and can be regarded as an extension of SW Chinese center, prominent even in the global scale.

Sheh Menglan and Su Pu (1987) made a special analysis of endemic Umbelliferae genera in China. They registered 19 such genera, but now only Sinolimprichtia, Cyclorhiza, Chaerophyllopsis, Harrysmithia, Sinodielsia, Dickinsia, Melanoscia- dium, and Changium can be regarded as generic Chinese endemics in the Umbelliferae. No new endemic genera have been separated in China since then. It seems, however, that some new genera can be described from China, based on the species currently treated in Peucedanum, Trachydium, Hymenidium and some other genera.

It is of interest to compare large Asiatic countries with high Umbelliferae biodiversity in average number of Umbelliferae species per genus. In China this ratio is the highest, near 6.0 (5.97), against Turkey with 4.38, Asiatic Russia (2.57), Kazakhstan (2.99), and India (3.02). These data could be interpreted as an indicator of the incomplete generic separation in China, a retension of traditional polymorphic genera. Indeed, some genera of Chinese Umbelliferae remain actually non monophyletic and critical (Peucedanum, Ligusticum, Tongoloa, Sinocarum, especially Trachydium, and so on).

\section{Acknowledgements}

This publication has been supported by grants from the Russian Foundation for basic research (RFBR, grants №№ 13-04-00648 and 16-04-00525, whereas the botanical trip to China by the grant from the National Geographic Society (U.S.A.). Author also thanks the curators and staff of all herbaria, which were visited during this investigation and marked in text, for working facilities and valuable help.

\section{REFERENCES / ЛИTEPATУРА}

Alava R. 1987. Ormosciadium, Tordylium, Ainsworthia, Ducrosia, Kalakia, Zosima, Kandaharia, Semenovia, Tricholaser, Malabaila, Trigonosciadium, Stenotaenia, Scrithacola. In: Rechinger, K.H. (ed.), Flora Iranica 162: 464-491, 507-519. Graz: Akademische Druck- und Verlagsanstalt.

Brummitt R. K., Powell C. E. 1992. The authors of plant names; a list of authors of scientific names of plants, with recommended forms of their names, including abbreviations. Royal Botanic gardens, Kew, 732 pp.

Chang Hotseng, Fu Kuntsun, Ho, Yehchi, Hsü Longjan, Li Yin, Liou Shoulu, Pu Fating, Shan Renhwa, Sheh Menglan, Shen Kuanmien, Wang Tiehseng, Yuan Changchi. 1979, 1985, 1992. Umbelliferae. In: Flora Reipublicae Popularis Sinicae. Eds. Shan Renhwa, Sheh Menglan. Beijing, 55(1): 1-300; 55(2): 1-268; 55(3): 1-255.

Chen Chihhsiung, Wang Jeenche. 2001. Revision of the genus Oreomyrrhis Endl. (Apiaceae) in Taiwan. Bot. Bull. Acad. Sinica (Taipei) 42 (4): 303-312.

Constance L. 1997. An instance of East-West confusion in Chinese Umbelliferae, or Arracacia out of Asia! Edinburgh J. Bot. 54 (1): 99-104.

Franchet $\boldsymbol{A}$. 1882. Catalogue des plantes recueilliés aux environs de Tche-fou. Mém. Soc. Sci. Natur. Cherbour, 3 sér. 4 (24): 193-276.

Franchet A. 1883. Plantae davidianae ex sinarum imperio. Pt. I. Nouv. Arch. Mus. Hist. Natur., sér. 2 6: 11-26.

Franchet A. 1885. Plantae davidianae ex sinarum imperio. Pt. 2. Nouv. Arch. Mus. Hist. Natur., sér. 2 8: $183-254$.

Franchet $\boldsymbol{A}$. 1888. Plantae davidianae ex sinarum imperio. Pt. 2. Nouv. Arch. Mus. Hist. Natur., sér. 2 10: 3-198.

Franchet A. 1894. Notes sur quelques Ombellifères du Yunnan. Bull. Soc. Phylom. Paris, sér. 8 6: 106-146.

Franchet $A$. 1895. Sur quelques plantes de la Chine occidentale. Bull. Mus. Hist. e Natur. (Paris) 1: 62-66.

Geldykhanov A. M. 1992. Umbelliferae of Turkmenistan flora. Ylym, Ashkhabad, 188 pp. [In Russian]. 
Gubanov I. A. 1993. Catalogue of type specimens of herbarium of the Moscow university (MW). Moscow, $155 \mathrm{pp}$. [In Russian].

Gubanov I. A., Bagdasarova T. V., Balandina T. P. 1998. Scientific heritage of the prominent Russian florists G. S. Karelin and I. P. Kirilov]. Moscow University Press, Moscow, 95 pp. [In Russian].

Gureeva I. I., Balashova V. F. 2015. Type specimens of Apiaceae in P. N. Krylov Herbarium. Sist. Notes Mater. Herb. P. N. Krylov Tomsk State Univ. 112: 3-13 [In Russian].

Gureeva I. I., Pimenov M. G., Balashova V. F. 2016. Two lectotypes of Apiaceae family in P. N. Krylov Herbarium (TK). Sist. Zametki Mater. Gerb. Krylova Tomsk. Gosud. Univ. [Systematic notes on the materials of P. N. Krylov Herbarium of Tomsk State University] 113: 37-40 [In Russian].

Hedge I. C., Lamond J. M. 1972. Sanicula, Astrantia, Actinolema, Lagoecia, Echinophora, Myrrhoides, Rhabdosciadium, Chaerophyllum, Grammosciadium, Anthriscus, Scandix, Coriandrum, Fuernrohria, Bifora, Bunium, Carum, Huetia, Stefanoffia, Muretia, Seseli, Oenanthe, Aethusa, Foeniculum, Anethum, Kundmannia, Trachydium, Hohenackeria, Trinia, Microsciadium, Olymposciadium, Cicuta, Cnidium, Ligusticum, Laser, Glaucosciadium, Elaeoselinum, Laserpitium, Thapsia, Exoacantha. In: Flora of Turkey and the East Aegean Islands. Ed. P. H. Davis. University Press, Edinburgh, 4: 289-292, 304-333, 342-351, 367-378, 380-381, 393, 418-420, 422, 425, 429-431, 513-518, 537-538.

Hedge I. C., Lamond J. M. 1987. Anisosciadium, Dicyclophora, Echinophora, Pycnocycla, Ergocarpon, Thecocarpus, Anthriscus, Chaerophyllum, Physocaulis, Rhabdosciadium, Scandix, Fuernrohria, Neocryptodiscus, Stewartiella, Hyalolaena, Berula, Diplotaenia, Korovinia, Oedibasis, Hohenackeria, Pinacantha. In: Flora Iranica. Ed. K. H. Rechinger. Akademische Druck- und Verlagsanstalt, Graz, 162: 65-96, 100-110, 162, 207-209, 213-214, 233-236, 305-306, 372-374, 436-438, 526-528.

Hiroe M. 1958. Umbelliferae of Asia (excluding Japan). Eikodo (Akira Imagawa), Kyoto, 1: 219 pp.

Jansen P. C. M. 1981. Spices, condiments and medicinal plants of Ethiopia: Their taxonomy and agricultural significance. Centre for Agricultural Publishing and Documentation, Wageningen, 1-32 pp.

Jarvis C. E. 1992. Seventy-two proposals for the conservation of types of selected Linnean generic names, the report of Subcommitee 3C on the lectotypification of Linnean generic names. Taxon 41: 552-583.

Jarvis C. E., Barrie F. R., Allan D. M., Reveal J. L. 1993. A list of Linnean generic names and their types. Regnum Vegetabilis 127: 1-100.

Jarvis C. E., Knees S. G. 1988. Linnean names in the genus Athamanta L. (Umbelliferae: Apioideae) and their typification. Taxon 37(2): 448-453.

Jarvis C. E., Reduron J.-P., Spencer M. A., Cafferty S. 2006. Typification of Linnean plant names in Apiaceae. Taxon 55(1): 207-216.

Jonsell B., Jarvis $\boldsymbol{C}$. E. (ed.) 2002. Lectotypifications of Linnean names for Flora Nordica (Brassicaceae - Apiaceae) Nordic J. Bot. 22(1): 67-86.

Kilpatrick J. 2014. Fathers of Botany. The discovery of Chinese plants by European missionaries. Kew \& Chicago. 254 pp.

Kitagawa M. 1960. Synoptical review of Umbelliferae from Japan, Korea and Manchuria. Bull. Nat. Sci. Mus. Tokyo 5: 1-35.

Kitagawa M. 1979. Neo-Lineamenta florae Manshuricae: or enumeration of the spontaneous vascular plants hitherto known from Manchuria (north-eastern China) together with their synonymy and distribution. J. Kramer, Hirschberg, 715 pp.

Kljuykov E. V., Pimenov M. G., Tikhomirov V. N. 1976. Revision of the genus Aulacospermum (Umbelliferae Apioideae) 2. Review of species. Byulleten MOIP. Otdel biologicheskiy [Bulletin of Moscow Society of Naturalists Biological series] 81 (5): 61-68 [In Russian].

Kljuykov E. V., Pimenov M. G., Tikhomirov V. N. 1977. Bunium capillifolium Kar. et Kir.: taxonomy, geography, ecology. Nauchney doklady Vysshey Shkoly. Biologicheskiye Nauki 10: 137-143.

Komarov V. L. 1907. Flora Manshuriae. Vol. 3. Pt. 1. In: Acta Horti Petropolitani. Trudy Imp. St.-Petersburg. Bot. Sada 25 (1): 1-334 [In Russian].

Korovin E .P. 1951. Ferula L., Oedibasis K.-Pol., Korovinia Nevski et Vved., Scaphespermum Korov. In: Flora SSSR [Flora of the USSR]. Ed. B. K. Schischkin. Akad. nauk SSSR, Moscow - Leningrad, 17: 62-141, 203-208, 211-214.

Koso-Poljanskyy B. M. 1920. Umbelliferae - Pachystereomae of Asiatic Russia. In: Acta Horti Petropolitani. Flora Aziatskoy Rossii. Ed. B. A. Fedtschenko. Iss. 15. Petrograd, 36(1): 1-120.

Kwak Myoung Hai, Lim Jina, Lee Byoun Gyoon, Grabovskaya-Borodina A. E., Illarionova I. D., Tatanov I. V. 2013. Korean type specimens of vascular plants deposited in Komarov Botanical Institute. National Institute of Biological Resources. Incheon, Korea. 255 pp.

Leute G.-H. 1969. Untersuchungen über den Verwandtschaftkreis der Gattung Ligusticum L. (Umbelliferae) 1. Teil. Ann. Naturhist. Mus. Wien 73: 55-98. 
Leute G.-H. 1970. Untersuchungen über den Verwandtschaftkreis der Gattung Ligusticum L. (Umbelliferae) 2 Teil. Ann. Naturhist. Mus. Wien 74: 457-519.

Li Jerhaur, Chen Chienwen, Yang Kuohcheng, Chung Shinwen, Chiou Wenliang (eds.) 2007. Type specimens in the herbarium of the Taiwan Forestry Research Institute. Taipei TFRI Extension series, 176: 1-204.

Linczevski I. A. 1950. Bupleurum L. In: Flora SSSR [Flora of the USSR]. Ed. B. K. Schischkin. Akad. nauk SSSR, Moscow - Leningrad, 16: 275-349.

Lowry P. P. II, Jones A. G. 1984. Systematics of Osmorhiza Raf. (Apiaceae: Apioideae). Ann. Missouri Bot. Gard. 71 (4): 1128-1171.

Menitski Ju. L. 2008. Apiaceae Lindl. In: Conspectus florae Caucasi. Ed. A. L. Takhtajan. KMK Scientific Press, St.-Petersburg - Moscow, 53-112 pp. [In Russian].

Mukherjee P. K. 1971. A note on the correct identity of Pimpinella radiata W. W. Sm. Indian Forester 97(1): $55-56$.

Mukherjee P. K., Constance L. 1993. Umbelliferae (Apiaceae) of India. Oxford \& IBH Publishing Co., New Delhi, Bombay, Calcutte, 279 pp.

Nicolas A. N., Plunkett G. M. 2009. The demise of subfamily Hydrocotyloideae (Apiaceae) and re-alignment of its genera across the entire order Apiales. Mol. Phylogenet. \& Evol. 53: 134-151.

Norman C. 1937. Cortia hookeri C. B. Clarke. J. Bot. 75: 93-96.

Pardó C. 1981. Estudio sistemático del género Seseli L. (Umbelliferae) in la peninsula Ibérica. Lazaroa 3: 163182.

Pimenov M. G. 1978. De generis Seseli L. notulae systematicae. II. Adumbratio specierum florae URSS. Novosti sistematiki vysshikh rasteniy [Novit. Syst. Pl. Vasc.] 15: 188-200.

Pimenov M. G. 1987. Umbelliferae. In: Vascular plants of the Soviet Far East. Ed. S. S. Charkevish. Vol. 2. Nauka, Leningrad, 203-277 pp. [In Russian].

Pimenov M. G. 1998. What is Peucedanum wolffianum (Umbelliferae)? Bot. Zhurn. (St. Petersburg) 83(2): 80-84 [In Russian].

Pimenov M. G., Baranova Ju. V. 1979. A conspectus of the species of the section Palaeonarthex Korov. of the genus Ferula L. Byulleten MOIP. Otdel biologicheskiy [Bulletin of Moscow Society of Naturalists Biological series] 84 (3): 82-92 [In Russian].

Pimenov M. G., Kljuykov E. V. 2000.Taxonomic revision of Pleurospermum and related genera of the Umbelliferae. II. The genera Pleurospermum, Pterocyclus, Trachydium, Keraymonia, Pseudotrachydium, Aulacospermum and Hymenolaena. Feddes Repert. 111(7-8): 517-534.

Pimenov M. G., Kljuykov E. V. 2000. Taxonomic revision of Pleurospermum and related genera of the Umbelliferae. III. The genera Physospermopsis and Hymenidium. Feddes Repert. 111(7-8): 535-552.

Pimenov M. G., Kljuykov E. V. 2015. Nomenclatural correction for a Chinese species of Conioselinum (Umbelliferae). Skvortsovia 2(1): 28-34.

Pimenov M. G., Kljuykov E. V., Ostroumova T. A. 2001. Towards a clarification in the taxonomy of Sino-Himalayan species of Selinum s. 1. (Umbelliferae) The genus Oreocome. Willdenowia 31(1): 101-124.

Pimenov M. G., Kljuykov E. V., Ostroumova T. A. 2003. A revision of Conioselinum Hoffm. (Umbelliferae) in the Old World. Willdenowia 33(2): 353-377.

Pimenov M. G., Sutorý K. 2014. Name typification of Umbelliferae taxa described by Joseph Franz Freyn. Plant Biosystems 148(5-6): 1102-1111.

Plunkett G. M., Chandler G. T., Lowry P. P., Pinney S. M., Sprenkle T. S. 2004. Recent advances in understanding Apiales and a revised classification. South Afr. J. Bot. 70(3): 371-381.

Polozhij A. V., Balaschova V. F. 1989.Types of taxa in the Herbarium nom. P. N. Krylov. Tomsk, 47 3p. [In Russian].

Pu Fating. 1998. New names in Chinese Umbelliferae. Novon 8(1): 70-71.

Reduron J.-P. 2007-2008. Ombellifères de France, 1-5. Bull. Soc. Bot. u Centre-Ouest, Nouv. e Sér., num. spéc. 26: 1-564; 27: 565-1142; 28: 1143-1726; 29: 1727-2348; 30: 2349-3004.

Reduron J.-P., Jarvis C. E. 1992. Angelica, Apium, Chaerophyllum. In: Seventy-two proposals for the conservation of types of selected Linnean generic names, the report of Subcommitee 3C on the lectotypification of Linnean generic names. Ed. D. H. Nicolson.Taxon 41(3): 552-583.

Saenz Lain C. 1981. Research on Daucus L. (Umbelliferae) An. Jard. Bot. Madrid 37(2): 481-533.

She Menglan, Pu Fading, Pan Zehui, Watson M.F., Cannon J.F.M., Holmes-Smith I., Kljuykov E. V., Phillippe L. R., Pimenov M. G. 2005. Apiaceae (Umbelliferae). In: Flora of China. Ed. Wu Zhengyi, R. H. Raven Vol. 14 (Apiaceae through Ericaceae). Missouri Botanical garden Press, Beijing: Science Press \& St. Louis, 1-205 pp.

Singh R. K. 2015. Lectotypification of four species of Indian Trachyspermum (Apiaceae). Telopea 18: $247-253$.

Spalik K. 1995. Typification of the Boissier names in Anthriscus Pers. (Apiaceae). Bot. J. Linnean Society 119: 77-86. 
Takhtajan A. L. 1957. On origin of temperate flora of Eurasia. Bot. Zhurn. (Leningrad) 42: 1635-1653.

Takhtajan A. L. 1969. Flowering plants, origin and dispersal. Oliver \& Boyd, Edinburgh, 310 pp.

Takhtajan A. L. 1970. Origin and dispersal of flowering plants. Nauka, Leningrad, 145 pp. [In Russian].

Takhtajan A. L. 1978. Floristic regions of the World. Nauka, Leningrad, 246 pp. [Engl. translation: 1988. Dehra Dun, Bishen Singh, Mohendra pal Singh].

Tardieu-Blot M.-L. 1967. Umbelliferae. In: Flore du Cambodge, du Laos et du Vietnam. Ed. M.-L. Tardieu-Blot. Muséum National d'Histoire Naturelle, Paris, 5: 1-77.

Thiers B. [continuously updated]. Index Herbariorum: A global directory of public herbaria and associated staff. New York Botanical Garden’s Virtual Herbarium. http://sweetgum.nybg.org/science/ih/.

Vinogradova V. M. 1997a. Type specimens of Apiaceae taxa from Middle Asia, kept in Herbarium of Komarov Botanical institute (LE). 1. Angelica-Hyalolaena. Bot. Zhurn. (St. Petersburg) 82 (3): 95-102 [In Russian].

Vinogradova V. M. 1997b. Type specimens of Apiaceae taxa from Middle Asia, kept in Herbarium of Komarov Botanical institute (LE). 2. Hymenolaena-Seselopsis. Bot. Zhurn. (St. Petersburg) 82 (4): 53-61[In Russian].

Vinogradova V. M. 1999. Type specimens of Apiaceae taxa from Middle Asia, kept in Herbarium of Komarov Botanical institute (LE). 3. Aegopodium-Eryngium. Bot. Zhurn. (St. Petersburg) 84 (3): 80-87 [In Russian].

Vinogradova V. M. 2001. Type specimens of Apiaceae taxa from Middle Asia, kept in Herbarium of Komarov Botanical institute (LE). 5. Elaeosticta-Prangos. Bot. Zhurn. (St. Petersburg) 86 (2): 50-57[In Russian].

Vinogradova V. M. 2002. Type specimens of Apiaceae taxa from Middle Asia, kept in Herbarium of Komarov Botanical institute (LE). 6. Reutera-Zozima. Bot. Zhurn. (St. Petersburg) 87 (6): 132-140 [In Russian].

Vinogradova $\boldsymbol{V}$. M. 2003. Type specimens of Apiaceae taxa from East Europe, kept in the Herbarium of Komarov Botanical Institute of RAS (LE). Bot. Zhurn. (St. Petersburg) 88 (8): 105-111.

Vinogradova V. M. 2004. Apiaceae. In: Catalogue of the type specimens of East Asian vascular plants in the Herbarium of the Komarov Botanical Institute (LE). Ed. V. I. Grubov. Part. 1. KMK Scientific Press, Moscow - St. Petersburg, 190 pp. [In Russian].

Vinogradova V. M. 2005. Type specimens of Apiaceae taxa from Siberia and North-Eastern Kazakhstan, kept in Herbarium of Komarov Botanical institute (LE) Aegopodium - Stenocoelium. Bot. Zhurn. (St. Petersburg) 90(6): 957-967 [In Russian].

Vinogradova $\boldsymbol{V}$. M. 2006. Type specimens of Apiaceae taxa from the Far East, kept in Herbarium of Komarov Botanical institute (LE) Angelica - Tilingia. Bot. Zhurn. (St. Petersburg) 91(8): 1280-1286 [In Russian].

Vinogradova V. M. 2007. Note on type material of Ledebouriella multiflora (Ladeb.) H. Wolff (Apiaceae). Novosti Sist. Vyssh. Rast. [Novit. Syst. Plant. Vasc.] 39: 260-272 [In Russian].

Vinogradova V. M. 2010. Apiaceae Lindl. In: Catalogue of the type specimens of East Asian vascular plants in the Herbarium of the Komarov Botanucal Institute (LE). Part 2 (China). KMK Scientific Press, Moscow - St. Petersburg, 49-53 [In Russian].

Wang Lisong. 2008a. Taxonomic revision of the Pternopetalum delavayi complex (Apiaceae). Ann. Bot. fennici 45(2): 105-112.

Wang Lisong. 2008b. The confusing identity of Pternopetalum molle (Apiaceae). Bot. J. Linnean Soc. 158(2): 274-295.

Watson M. F. 1996. Notes relating to the flora of Bhutan: XXXVI Umbelliferae. I. Edinburgh J. Bot. 53(1): 127-144.

Watson M. F. 1998. Notes relating to the flora of Bhutan: XXXVI Umbelliferae. II. Edinburgh J. Bot. 55(3): 367-415.

Wijnheimer E. H. M., Brandenburg W. A., Jarvis C. E. 1988. Lectotypification of Daucus carota L. (Umbelliferae). Taxon 37: 175-184.

Wolff H. 1910. Umbelliferae-Apioideae-Bupleurum, Trinia et reliquae Ammineae heteroclitae. In: Engler, A. (ed.), Die Pflanzenreich 43 (IV, 228): 214 S. Berlin: Wilhelm Engelmann.

Wolff H. 1913. Umbelliferae - Saniculoideae. In: Das Pflanzenreich. Ed. A. Engler. Wilhelm Engelmann, Berlin, 61(IV, 228): 1-305.

Wolff H. 1922. Umbelliferae. In: Limpricht, Botanische Reisen in den Hochgebirgen Chinas und Ost-Tibets. Repert. Spec. Nov. Regni Veget. 12: 447-453.

Wolff H. 1924a. Umbelliferae novae asiaticae. I. Repert. Spec. Nov. Regni Veget. 19(16-21): 309-312.

Wolff H. 1924b. Changium, genus novum Umbelliferarum Chekiangense. Repert. Spec. Nov. Regni Veget. 19(1621): 314-315.

Wolff H. 1925a. Neue Umbelliferen - Gattungen aus Ostasien. Notizbl. Königl. Bot. Gart. Mus. Berlin-Dahlem 9: 275-280.

Wolff H. 1925b. Pleurosperma nova Tibetiae orientalis. Repert. Spec. Nov. Regni Veget. 21: 241-244.

Wolff H. 1925c. Umbelliferae novae asiaticae. Repert. Spec. Nov. Regni Veget. 21: 244-249.

Wolff H. 1926. Plantae sinenses a Dre. H. Smith annis 1921-22 lectae. XVI. Umbelliferae. Acta Horti Gothoburg. 
2: $288-328$.

Wolff H. 1927. Umbelliferae - Apioideae - Ammineae - Carinae, Ammineae-novemjugatae et genuinae. In: Das Pflanzenreich. Ed. A. Engler. Wilhelm Engelmann, Berlin, 90(VI, 228): 1-398.

Wolff H. 1929a. Umbelliferae asiaticae novae relictae. I. Repert. Spec. Nov. Regni Veget. 27: 112-128.

Wolff H. 1929b. Umbelliferae asiaticae novae relictae. II. Repert. Spec. Nov. Regni Veget. 27: 179-192.

Wolff H. 1930a. Umbelliferae asiaticae novae relictae. III. Repert. Spec. Nov. Regni Veget. 27: 301-335.

Wolff H. 1930b. Umbelliferae asiaticae novae relictae. IV. Repert. Spec. Nov. Regni Veget. 28: 109-112.

Wolff H. 1934. Umbelliferae asiaticae novae relictae. V. Repert. Spec. Nov. Regni Veget. 33: 75-80, 244-251.

Wörz A. 1999. A taxonomic index of the species of Eryngium L. (Apiaceae: Saniculoideae). Stuttgarter Beitr. Naturkunde, A 596: 48.

Wörz A. 2011. Revision of Eryngium L. (Apiaceae - Saniculoideae): General part and Palaeoarctic species. Bibliotheca Botanica (Stuttgart: Schweizerbart Science Publishers) 159: 498 pp.

Wu Zhengyi, Raven P., Hong Deyuan (eds.) 2006. Flora of China. Illustrations 14. Missouri Botanic Garden Press, St. Louis, 725 pp.

Xu Zhaoran, Nicolson D. H. 1992. Don't abbreviate Chinese names. Taxon 43(3): 499-504.

Yurtzeva O. V. 1995. On the system of Pimpinella L. subgenus Tragium (Spreng.) Reichenb. (Umbelliferae Juss.). Byulleten MOIP. Otdel biologicheskiy [Bulletin of Moscow Society of Naturalists Biological series] 100(3): 61-72. 